Supporting Information

\title{
Dearomatization of Unactivated Arenes via Catalytic Hydroalkylation
}

Kelly A. McDaniel, Anna R. Blood, Gavin C. Smith, Nathan T. Jui*

njui@emory.edu

Department of Chemistry and Winship Cancer Institute, Emory University, Atlanta, GA 30322

I. General Information............................................................

I-A. General Reagent Information......................................

I-B. General Analytical Information..........................................

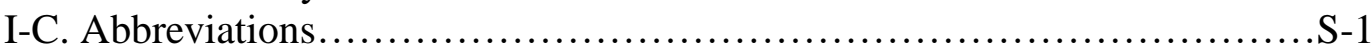

I-D. General Photoredox Reaction Setup.............................

II. General Procedures................................................................

II-A. General reductive amination procedure..........................

II-B. General acylation procedure...................................

II-C. General dearomative spirolactamization procedure $1 \ldots \ldots \ldots \ldots \ldots \ldots \ldots$. 3

II-C. General dearomative spirolactamization procedure $2 \ldots \ldots \ldots \ldots \ldots \ldots \ldots \ldots$. -3

III. Optimization Details........................................................

III-A. Optimization Procedure........................................

III-B. Optimization Table............................................

IV. Deuterium Labeling Study......................................................5-5

V. Electrochemical Measurements................................................S

VI. Computational Details..............................................................8

VII. Preparation of Starting Materials.............................................

VIII. Preparation of Spirolactam Products...........................................S-39

VIII-A. Derivatization.................................................. 54

IX. $\quad$ NMR Spectra...............................................................S-58 


\section{General Information}

\section{I-A. General Reagent Information}

Solvents used in anhydrous reactions were purified by passing over activated alumina and storing under argon. Reagents were purchased from Sigma-Aldrich, Alfa Aesar, Acros Organics, CombiBlocks, Oakwood Chemicals, Astatech, and TCI America and used as received, unless stated otherwise. Organic solutions were concentrated under reduced pressure on a rotary evaporator using a water bath. Chromatographic purification of products was accomplished using forced-flow chromatography on 230-400 mesh silica gel. Preparative thin-layer chromatography (PTLC) separations were carried out on $1000 \mu \mathrm{m}$ SiliCycle silica gel F-254 plates. Thin-layer chromatography (TLC) was performed on $250 \mu \mathrm{m}$ SiliCycle silica gel F-254 plates. Visualization of the developed chromatogram was performed by fluorescence quenching or staining using $\mathrm{KMnO}_{4}$, p-anisaldehyde, or ninhydrin stains. All photoredox reactions were set up on the bench top and conducted under nitrogen atmosphere while subject to irradiation from blue LEDs, unless stated otherwise (LED wholesalers PAR38 Indoor Outdoor 16-Watt LED Flood Light Bulb, Blue; or Hydrofarm ${ }^{\circledR}$ PPB1002 PowerPAR LED Bulb-Blue 15W/E27 (available from Amazon)). Solvent was degassed by sonication under mild vacuum for 15 minutes. Photoredox catalysts 3DPAFIPN, 3DPA2FBN, $5 \mathrm{CzBn}, 4 \mathrm{CzIPN}$, and [ $\left.\operatorname{Ir}(\mathrm{ppy})_{2} \mathrm{dtbbpy}\right] \mathrm{PF}_{6}$ were prepared according to literature procedures. ${ }^{1,2}$

\section{I-B. General Analytical Information}

Unless otherwise noted, all yields refer to chromatographically and spectroscopically $\left({ }^{1} \mathrm{H}\right.$ NMR) homogenous materials. New compounds were characterized by ${ }^{1} \mathrm{H}$ NMR, ${ }^{13} \mathrm{C}$ NMR, and LRMS. ${ }^{1} \mathrm{H}$ and ${ }^{13} \mathrm{C}$ NMR spectra were obtained from the Emory University NMR facility and recorded on a Bruker Avance III HD 600 equipped with cryo-probe (600 MHz), Bruker NEO 400 (400 MHz), INOVA $600(600 \mathrm{MHz})$, INOVA $500(500 \mathrm{MHz})$, INOVA 400 (400 MHz), or VNMR 400 (400 $\mathrm{MHz}$ ) and are internally referenced to residual protio solvent signals. Data for ${ }^{1} \mathrm{H}$ NMR are reported as follows: chemical shift $(\mathrm{ppm})$, multiplicity $(\mathrm{s}=$ singlet, $\mathrm{d}=$ doublet, $\mathrm{t}=$ triplet, $\mathrm{q}=$ quartet, $\mathrm{m}=$ multiplet, $\mathrm{dd}=$ doublet of doublets, $\mathrm{dt}=$ doublet of triplets, $\mathrm{ddd}=$ doublet of doublet of doublets, dtd= doublet of triplet of doublets, $\mathrm{b}=$ broad, etc.), coupling constant $(\mathrm{Hz})$, integration, and assignment, when applicable. Data for decoupled ${ }^{13} \mathrm{C}$ NMR are reported in terms of chemical shift and multiplicity when applicable. Gas Chromatography Mass Spectrometry (GCMS) was performed on an Agilent 5977A mass spectrometer with an Agilent 7890A gas chromatography inlet. Liquid Chromatography Mass Spectrometry (LCMS) was performed on an Agilent 6120 mass spectrometer with an Agilent 1220 Infinity liquid chromatography inlet.

\section{I-C. Abbreviations \\ $\mathrm{CV}=$ cyclic voltammogram \\ $\mathrm{DBU}=1,8$-diazabicyclo[5.4.0]undec-7-ene \\ $\mathrm{DCM}=$ dichloromethane}

\footnotetext{
${ }^{1}$ Speckmeier, E.; Fischer, T.; Zeitler, K. A Toolbox Approach to Construct Broadly Applicable Metal-Free Catalysts for Photoredox Chemistry: Deliberate Tuning of Redox Potentials and Importance of Halogens in DonorAcceptor Cyanoarenes. J. Am. Chem. Soc. 2018, 140, 15354-15365.

${ }^{2}$ Slinker, J. D.; Gorodetsky, A. A.; Lowry, M. S.; Wang, J.; Parker, S.; Rohl, R.; Bernhard, S.; Malliaras, G. G. Efficient Yellow Electroluminescence from a Single Layer of a Cyclometalated Iridium Complex. J. Am. Chem. Soc. 2004, 126, 2763-2767.
} 
DIPEA = diisopropylethylamine

$\mathrm{Et}_{2} \mathrm{O}=$ diethyl ether

EtOAc $=$ ethyl acetate

GCMS = gas chromatography mass spectrometry

HPLC $=$ high performance liquid chromatography

LCMS = liquid chromatography mass spectrometry

$m \mathrm{CPBA}=$ meta-chloroperoxybenzoic acid

$\mathrm{MeCN}=$ acetonitrile

$\mathrm{MeOH}=$ methanol

SCE $=$ saturated calomel electrode

TFA $=$ trifluoroacetic acid

$\mathrm{THF}=$ tetrahydrofuran

$\mathrm{TLC}=$ thin layer chromatography

\section{I-D. General Photoredox Reaction Setup}

To run multiple reactions (for optimization), an appropriately sized 3D printed carousel was used, which exposed the reactions to the blue light evenly (photo 1). A $15 \mathrm{~W}$ LED array lamp was used as a blue light source (photo 2,3). These lamps were routinely used for up to 12 reactions at a time (photo 2,3). The reactions were cooled with a line of compressed air (photo 2,3). The blue LEDs were positioned approximately 6 inches above the reaction vials to get good light coverage without overheating the reactions (photo 2,3). Reactions run at elevated temperatures were irradiated in a shallow oil bath (photo 4,5).

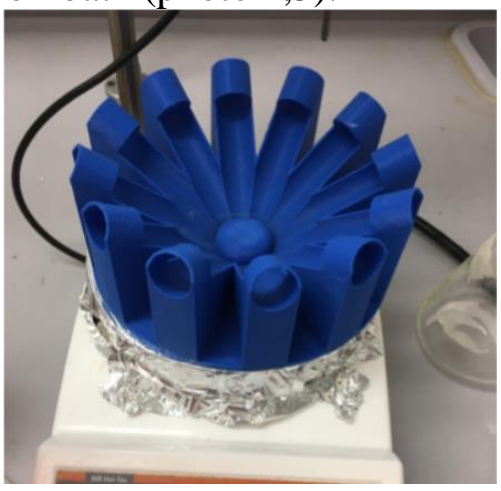

Photo 1

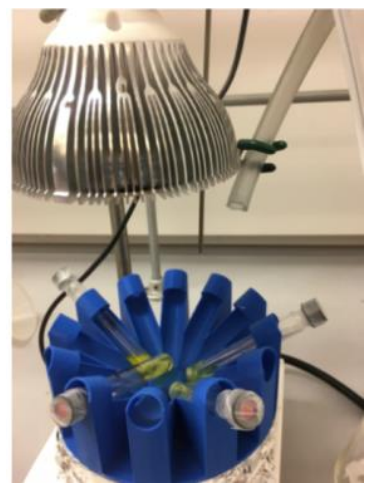

Photo 2

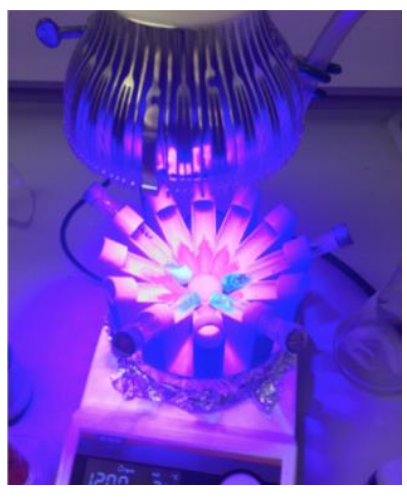

Photo 3

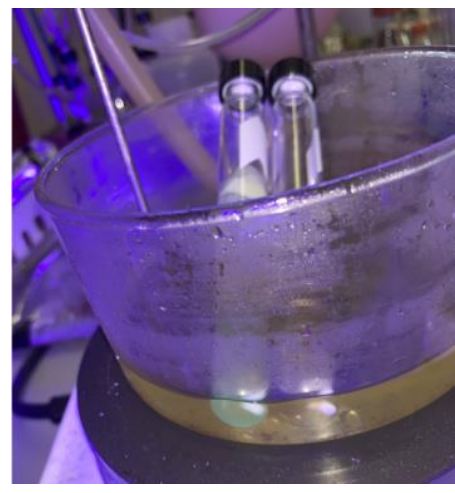

Photo 4

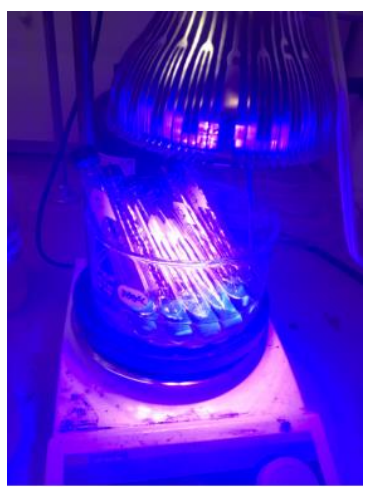

Photo 5 


\section{General Procedures}

\section{II-A. General Reductive Amination Procedure}

To a round bottomed flask charged with benzaldehyde (1.0 equiv) was added $\mathrm{MeOH}(0.2 \mathrm{M})$ and primary amine. After stirring for $2-16$ hours, $\mathrm{NaBH}_{4}$ (1.5 equiv) was added slowly, and the resultant mixture was stirred until bubbling ceased. The reaction mixture was quenched with $1 \mathrm{M}$ $\mathrm{NaOH}(\mathrm{aq})$ and extracted with EtOAc (3x). The combined organic layers were washed with $1 \mathrm{M}$ $\mathrm{HCl}$ (aq), and the resulting aqueous layer was brought to $\mathrm{pH} 14$ with $2 \mathrm{M} \mathrm{NaOH}$ (aq) or $50 \% \mathrm{KOH}$ (aq) and extracted with EtOAc (3x). The combined organic layers were dried over $\mathrm{MgSO}_{4}$ or $\mathrm{Na}_{2} \mathrm{SO}_{4}$, filtered, and concentrated under reduced pressure to afford the desired product.

\section{II-B. General Acylation Procedure}

To a round bottomed flask charged with secondary amine (1.0 equiv) was added $\mathrm{CH}_{2} \mathrm{Cl}_{2}(0.1 \mathrm{M})$, $\mathrm{Et}_{3} \mathrm{~N}$ (1.2 equiv), and chloroacetyl chloride (1.2 equiv). After stirring for 1-2 hours, the reaction mixture was quenched with $\mathrm{MeOH}$, diluted with $\mathrm{H}_{2} \mathrm{O}$, and extracted with $\mathrm{CH}_{2} \mathrm{Cl}_{2}$ (3x). The combined organic layers were dried over $\mathrm{MgSO}_{4} \mathrm{Or} \mathrm{Na}_{2} \mathrm{SO}_{4}$, filtered, and concentrated under reduced pressure. The crude residue was purified on silica using the indicated solvent mixture, if necessary, to afford the desired product.

\section{II-C. General Dearomative Spirolactamization Procedure 1}

A $20 \mathrm{~mL}$ screw-top test tube was charged with 3DPAFIPN (16.2 $\mathrm{mg}, 0.025 \mathrm{mmol}, 5 \mathrm{~mol} \%)$, and substrate $(0.5 \mathrm{mmol}, 1.0$ equiv). The tube was equipped with a stir bar and sealed with a PTFE/silicon septa. The atmosphere was exchanged by applying vacuum and backfilling with nitrogen (this process was conducted a total of three times). Under nitrogen atmosphere, DIPEA ( $0.26 \mathrm{~mL}, 1.5 \mathrm{mmol}, 3.0$ equiv) was added via syringe, followed by degassed solvent $(5 \mathrm{~mL}$ of each $\mathrm{MeCN}$ and $\mathrm{H}_{2} \mathrm{O}$ to give a $0.05 \mathrm{M}$ solution). The resulting mixture was stirred at $50{ }^{\circ} \mathrm{C}$ for 16 $\mathrm{h}$ under irradiation by blue LEDs, unless noted otherwise. The reaction was then extracted with EtOAc (3x), dried over $\mathrm{MgSO}_{4}$ or $\mathrm{Na}_{2} \mathrm{SO}_{4}$, and concentrated under reduced pressure. The crude residue was purified as indicated to afford the desired product (1,4-diene products stain strongly with $\mathrm{KMnO}_{4}$ ).

\section{II-D. General Dearomative Spirolactamization Procedure 2}

A $20 \mathrm{~mL}$ screw-top test tube was charged with 3DPAFIPN (16.2 mg, $0.025 \mathrm{mmol}, 5 \mathrm{~mol} \%$ ), and substrate $(0.5 \mathrm{mmol}, 1.0$ equiv). The tube was equipped with a stir bar and sealed with a PTFE/silicon septa. The atmosphere was exchanged by applying vacuum and backfilling with nitrogen (this process was conducted a total of three times). Under nitrogen atmosphere, DIPEA $(0.26 \mathrm{~mL}, 1.5 \mathrm{mmol}, 3.0$ equiv) was added via syringe, followed by degassed solvent ( $5 \mathrm{~mL}$ of each $\mathrm{MeCN}$ and $\mathrm{H}_{2} \mathrm{O}$ to give a $0.05 \mathrm{M}$ solution). The resulting mixture was stirred at $50{ }^{\circ} \mathrm{C}$ for 16 $\mathrm{h}$ under irradiation by blue LEDs, unless noted otherwise. The reaction was then extracted with EtOAc (3x), dried over $\mathrm{MgSO}_{4}$ or $\mathrm{Na}_{2} \mathrm{SO}_{4}$, and concentrated under reduced pressure. In order to deprotect the $\mathrm{HDH}$ byproduct that is sometimes difficult to separate from the dearomatized product via chromatography, the crude residue was dissolved in $50 \%$ (v/v) TFA $/ \mathrm{CH}_{2} \mathrm{Cl}_{2}(0.1 \mathrm{M})$ for 16 hours. The reaction was quenched with saturated $\mathrm{NaHCO}_{3}$ (aq) then extracted with EtOAc (3x), dried over $\mathrm{MgSO}_{4}$ or $\mathrm{Na}_{2} \mathrm{SO}_{4}$, and concentrated under reduced pressure. The crude residue was purified as indicated to afford the desired product (1,4-diene products stain strongly with $\mathrm{KMnO}_{4}$ ). 


\section{Optimization Details}

\section{III-A. Optimization Procedure}

An $8 \mathrm{~mL}$ screw-top test tube was charged with photocatalyst $(0.005 \mathrm{mmol}, 5 \mathrm{~mol} \%)$ and $N$-benzyl$N$-(tert-butyl)-2-chloroacetamide (S39) $(24.0 \mathrm{mg}, 0.1 \mathrm{mmol}, 1.0$ equiv). The tube was equipped with a stir bar and sealed with a PTFE/silicon septa. The atmosphere was exchanged by applying vacuum and backfilling with nitrogen (this process was conducted a total of three times). Under nitrogen atmosphere, DIPEA ( $52 \mu \mathrm{L}, 0.3 \mathrm{mmol}, 3$ equiv) was added via syringe, followed by degassed solvent. The resulting mixture was stirred at the indicated temperature for $16 \mathrm{~h}$ under irradiation by blue LEDs, unless noted otherwise. The reaction was then extracted with ethyl acetate $(3 \mathrm{x})$ and concentrated under reduced pressure. $\mathrm{CDCl}_{3}$ and an internal standard of dibromomethane $(7 \mu \mathrm{L}, 0.1 \mathrm{mmol})$ were added. The sample was analyzed by ${ }^{1} \mathrm{H}$ NMR $(\mathrm{d} 1=5 \mathrm{~s})$, and the integral values were used to calculate the data given in the Optimization Table.

\section{III-B. Optimization Table}

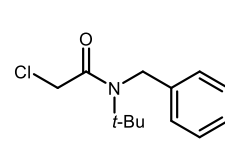

S39

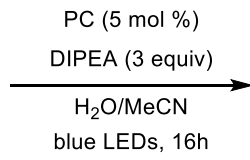

blue LEDs, $16 \mathrm{~h}$

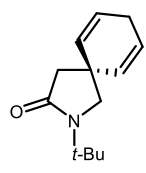

19

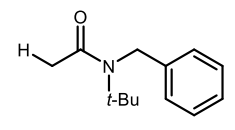

HDH

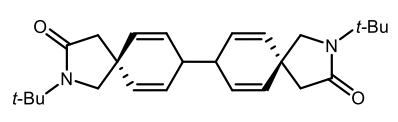

Dimer

\begin{tabular}{|c|c|c|c|c|c|c|c|c|c|}
\hline Entry & PC & Concentration & Temperature & $\%$ water & S39 & 19 & $\mathrm{HDH}$ & Dimer & \\
\hline 1 & 3DPAFIPN & $0.05 \mathrm{M}$ & $23^{\circ} \mathrm{C}$ & $50 \%$ & - & 74 & 25 & - & \\
\hline 2 & 3DPA2FBN & $0.05 \mathrm{M}$ & $23^{\circ} \mathrm{C}$ & $50 \%$ & - & 29 & 14 & 44 & \\
\hline 3 & $5 \mathrm{CzBN}$ & $0.05 \mathrm{M}$ & $23^{\circ} \mathrm{C}$ & $50 \%$ & - & 34 & 65 & - & \\
\hline 4 & 4CzIPN & $0.05 \mathrm{M}$ & $23^{\circ} \mathrm{C}$ & $50 \%$ & 78 & 16 & 7 & - & \\
\hline 5 & {$\left[\operatorname{lr}(\mathrm{ppy})_{2} \mathrm{dtbbpy}\right] \mathrm{PF} \mathrm{F}_{6}$} & $0.05 \mathrm{M}$ & $23^{\circ} \mathrm{C}$ & $50 \%$ & - & 42 & 58 & - & \\
\hline 6 & 3DPAFIPN & $0.1 \mathrm{M}$ & $23^{\circ} \mathrm{C}$ & $50 \%$ & - & 67 & 31 & - & \\
\hline 7 & 3DPAFIPN & $0.033 \mathrm{M}$ & $23^{\circ} \mathrm{C}$ & $50 \%$ & - & 74 & 24 & - & \\
\hline 8 & 3DPAFIPN & $0.05 \mathrm{M}$ & $0^{\circ} \mathrm{C}$ & $50 \%$ & - & 52 & 50 & - & \\
\hline 9 & 3DPAFIPN & $0.05 \mathrm{M}$ & $40^{\circ} \mathrm{C}$ & $50 \%$ & - & 79 & 21 & - & \\
\hline 10 & 3DPAFIPN & $0.05 \mathrm{M}$ & $50^{\circ} \mathrm{C}$ & $50 \%$ & - & 83 & 18 & - & optimized conditions \\
\hline 11 & 3DPAFIPN & $0.05 \mathrm{M}$ & $60^{\circ} \mathrm{C}$ & $50 \%$ & - & 79 & 19 & - & \\
\hline 12 & 3DPAFIPN & $0.05 \mathrm{M}$ & $50^{\circ} \mathrm{C}$ & $0 \%$ & - & 42 & 57 & - & \\
\hline 13 & 3DPAFIPN & $0.05 \mathrm{M}$ & $50^{\circ} \mathrm{C}$ & $10 \%$ & - & 39 & 60 & - & \\
\hline 14 & 3DPAFIPN & $0.05 \mathrm{M}$ & $\begin{array}{c}50^{\circ} \mathrm{C} \\
\text { controls }\end{array}$ & $25 \%$ & - & 54 & 44 & - & \\
\hline 15 & - & $0.05 \mathrm{M}$ & $23^{\circ} \mathrm{C}$ & $25 \%$ & 100 & - & - & - & no PC \\
\hline 16 & 3DPAFIPN & $0.05 \mathrm{M}$ & $23^{\circ} \mathrm{C}$ & $25 \%$ & 97 & - & - & - & no reductant \\
\hline 17 & 3DPAFIPN & $0.05 \mathrm{M}$ & $23^{\circ} \mathrm{C}$ & $25 \%$ & 99 & - & - & - & no light \\
\hline
\end{tabular}




\section{Deuterium Labeling Study}

\section{General Deuterium Labeling Procedure}

An $8 \mathrm{~mL}$ screw-top test tube was charged with 3DPAFIPN ( $3.2 \mathrm{mg}, 0.005 \mathrm{mmol}, 5 \mathrm{~mol} \%$ ) and substrate $(0.1 \mathrm{mmol}, 1.0$ equiv). The tube was equipped with a stir bar and sealed with a PTFE/silicon septa. The atmosphere was exchanged by applying vacuum and backfilling with nitrogen (this process was conducted a total of three times). Under nitrogen atmosphere, degassed solvent was added via syringe ( $1 \mathrm{~mL}$ of each $\mathrm{MeCN}$ and $\mathbf{D}_{2} \mathbf{O}$ to give a $0.05 \mathrm{M}$ solution), followed by DIPEA $\left(0.26 \mathrm{~mL}, 1.5 \mathrm{mmol}, 3.0\right.$ equiv). The resulting mixture was stirred at $50{ }^{\circ} \mathrm{C}$ for $16 \mathrm{~h}$ under irradiation by blue LEDs. The reaction was then extracted with ethyl acetate $(3 \mathrm{x})$ and concentrated. $\mathrm{CDCl}_{3}$ was added and the sample was analyzed by ${ }^{1} \mathrm{H} \mathrm{NMR}(\mathrm{d} 1=5 \mathrm{~s})$ to determine the $\% \mathrm{D}$ incorporation at the indicated position.

\section{Experiment 1 - Deuterium incorporation in dearomatized spirolactam}

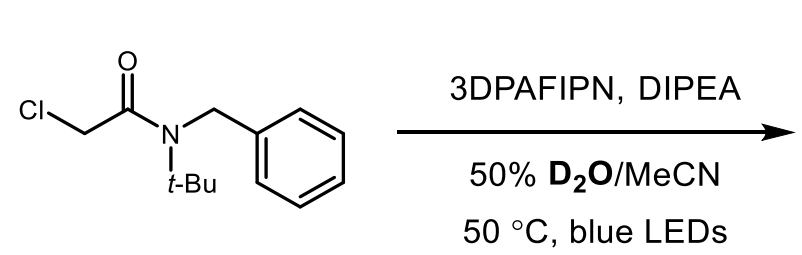

$N$-benzyl- $N$-(tert-butyl)-2-chloroacetamide (S39)

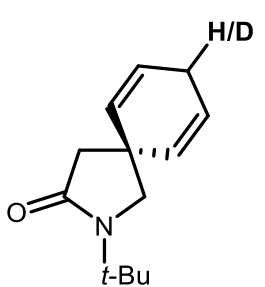

Deuterium Labeling Procedure. ${ }^{1} \mathrm{H}$ NMR analysis showed $>95 \%$ deuterium incorporation.

\section{Experiment 2 - Deuterium incorporation in hydrodehalogenation byproduct}
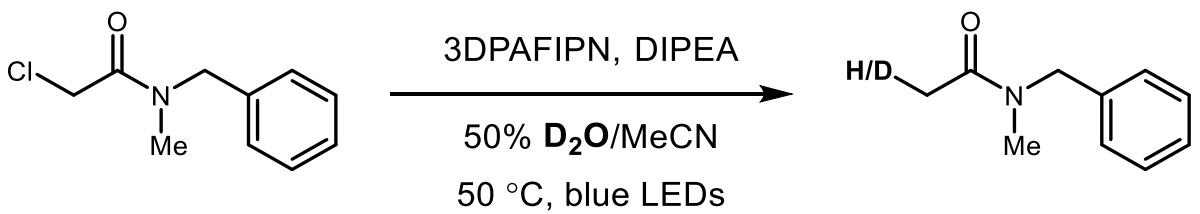

$N$-benzyl-2-chloro- $N$-methylacetamide (S39) $(24.0 \mathrm{mg}$ ) was subjected to the General Deuterium Labeling Procedure. ${ }^{1} \mathrm{H}$ NMR analysis showed $88 \%$ deuterium incorporation.

\section{Electrochemical Measurements}

Electrochemical potentials were obtained with a standard set of conditions according to literature procedure. ${ }^{3}$ Cyclic voltammograms (CVs) were collected with a VersaSTAT 4 Potentiostat. Samples were prepared with $0.05 \mathrm{mmol}$ of substrate in $10 \mathrm{~mL}$ of $0.1 \mathrm{M}$ tetra- $n$-butylammonium hexafluorophosphate in dry, degassed acetonitrile. Measurements employed a glassy carbon working electrode, platinum wire counter electrode, $3 \mathrm{M} \mathrm{NaCl}$ silver-silver chloride reference electrode, and a scan rate of $100 \mathrm{mV} / \mathrm{s}$. Reductions were measured by scanning potentials in the negative direction and oxidations in the positive direction; the glassy carbon electrode was polished between each scan. Data was analyzed using Microsoft Excel by subtracting a background current prior to identifying the maximum current $\left(\mathrm{C}_{\mathrm{p}}\right)$ and determining the potential

\footnotetext{
${ }^{3}$ Roth, H. G.; Romero, N. A.; Nicewicz, D. A. Experimental and Calculated Electrochemical Potentials of Common Organic Molecules for Applications to Single-Electron Redox Chemistry. Synlett. 2016, 27, 714-723.
} 
$\left(\mathrm{E}_{\mathrm{p} / 2}\right)$ at half this value $\left(\mathrm{C}_{\mathrm{p} / 2}\right)$. The obtained value was referenced to $\mathrm{Ag} \mid \mathrm{AgCl}$ and converted to SCE by subtracting $0.035 \mathrm{~V}$.

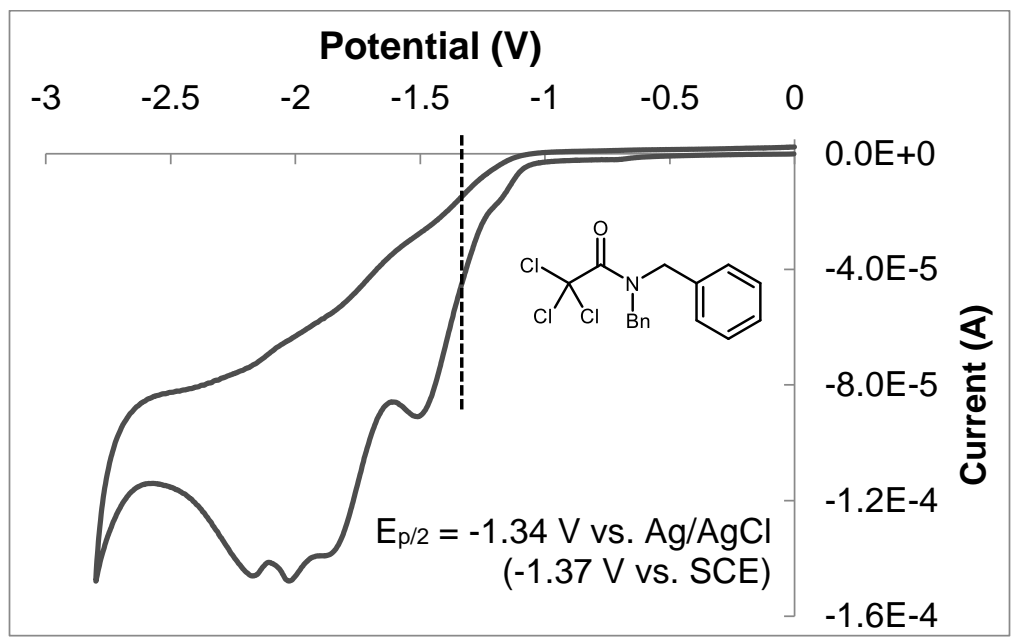

Figure S1: CV of $N, N$-dibenzyl-2,2,2-trichloroacetamide (S50)

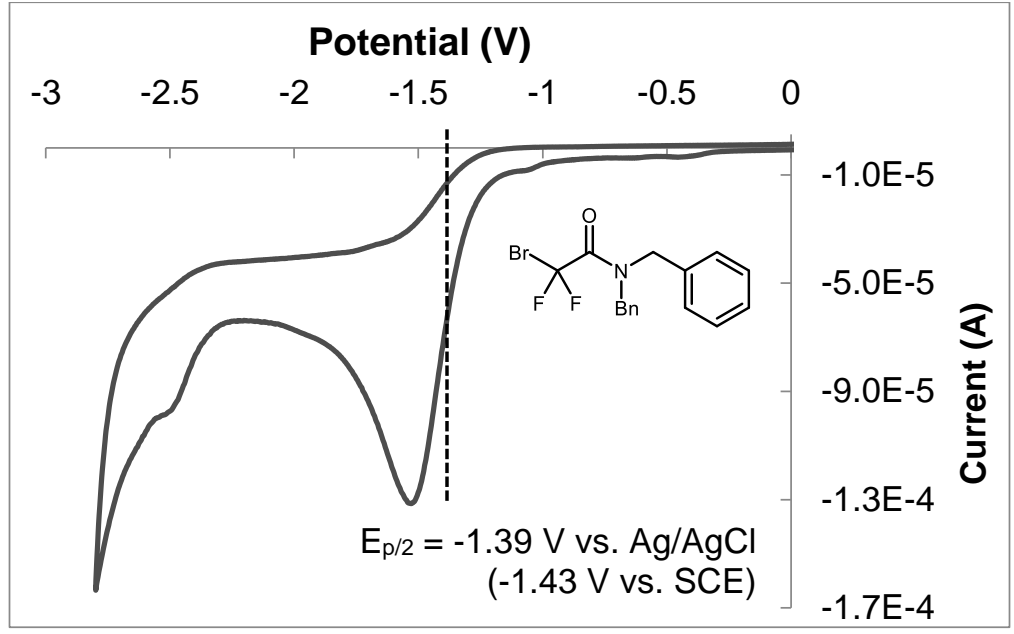

Figure S2: CV of $N, N$-dibenzyl-2-bromo-2,2-difluoroacetamide (S49)

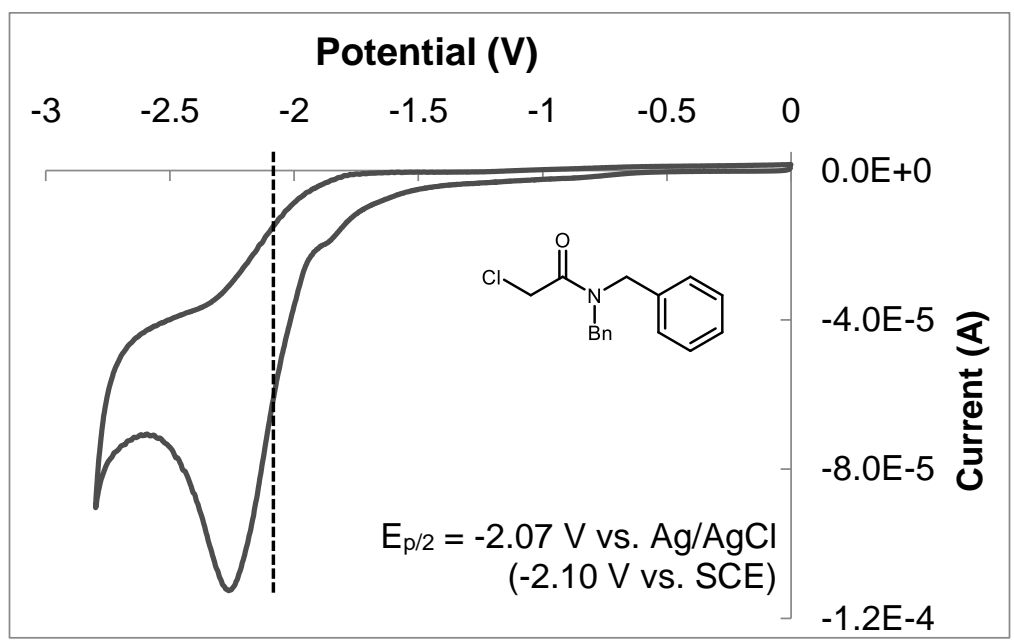

Figure S3: CV of $N, N$-dibenzyl-2-chloroacetamide (S46) 


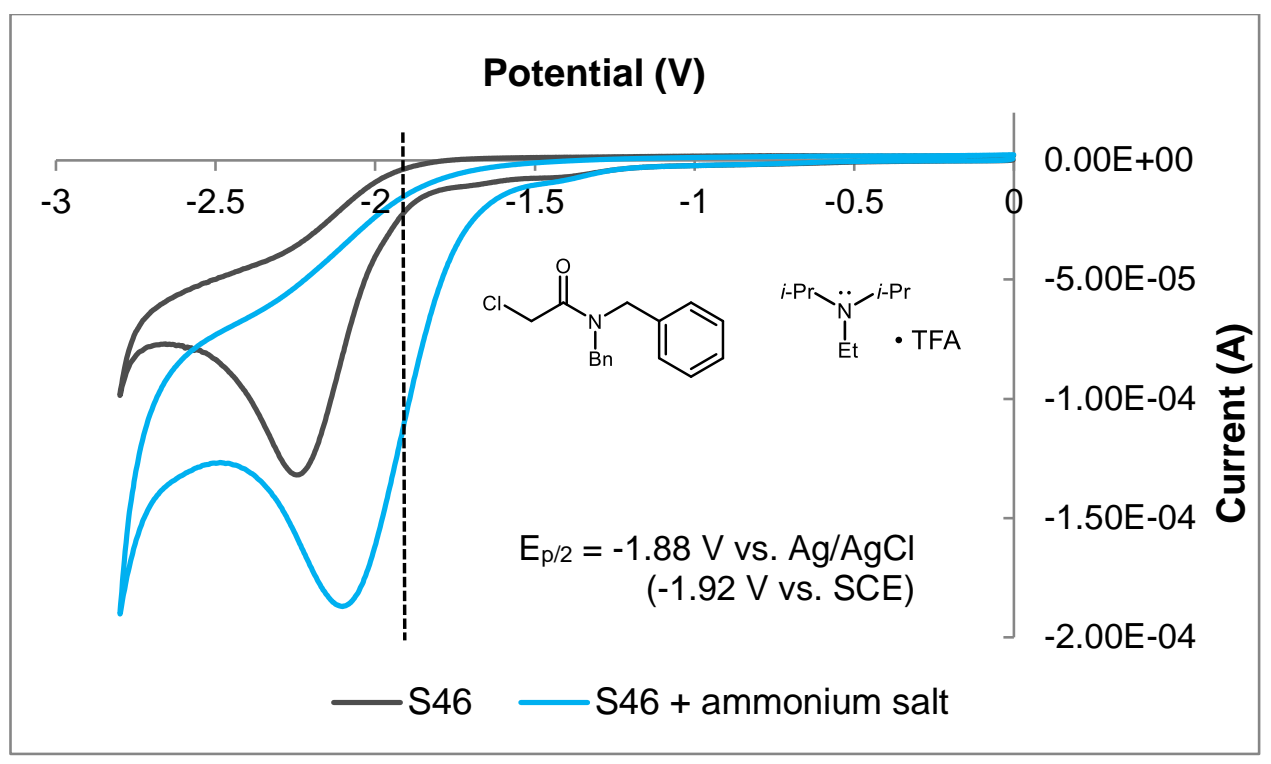

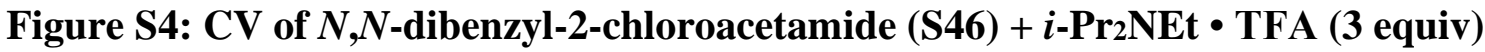

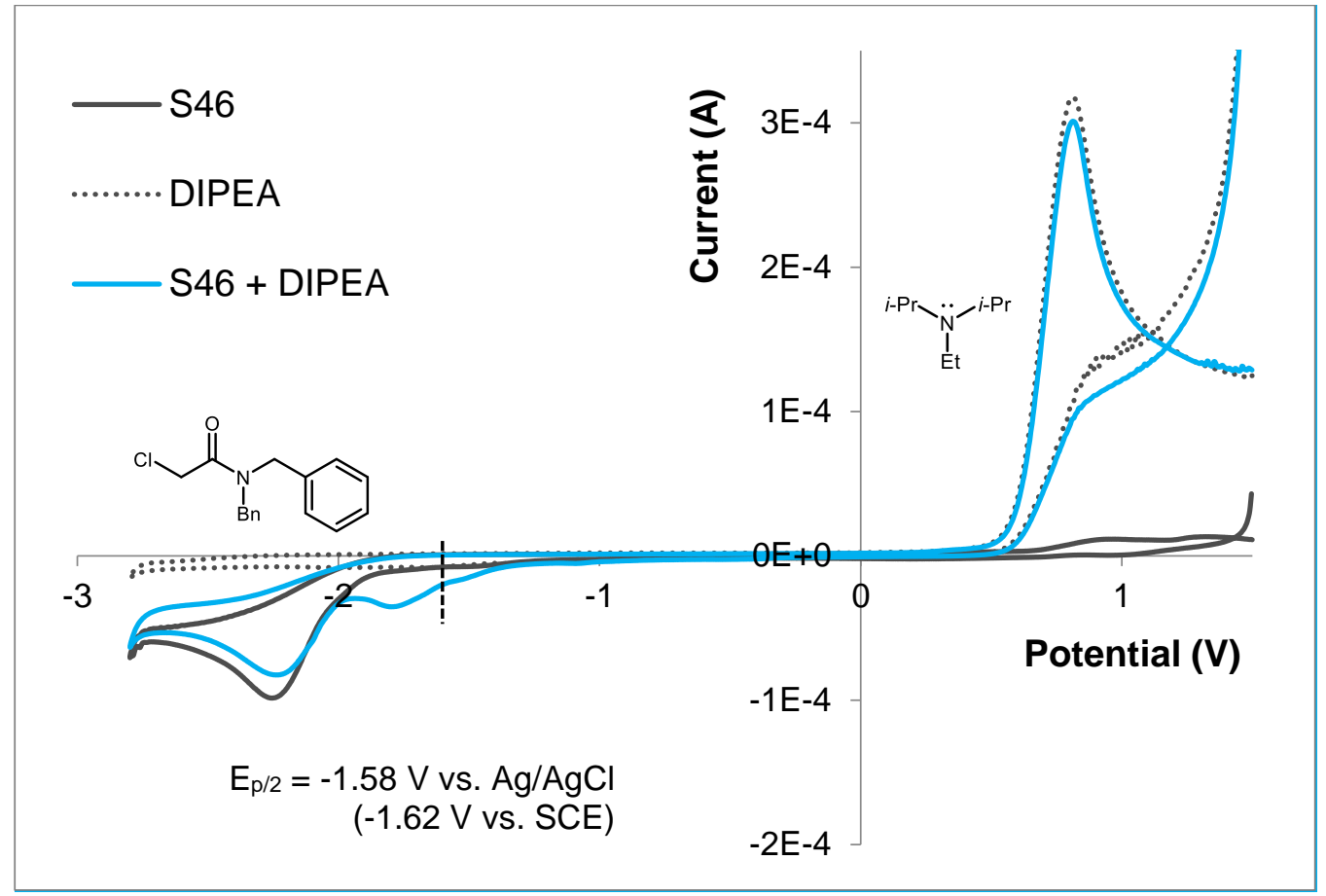

Figure S5: CV of $N, N$-dibenzyl-2-chloroacetamide (S46) $+i$-Pr $2 \mathrm{NEt}$ (3 equiv) 


\section{Computational Details}

All DFT calculations were carried out using the Gaussian 9 software package ${ }^{4}$ at the (U)B3LYP or R(B3LYP $)^{5}$ level of theory with the $6-311+\mathrm{G}(\mathrm{d}, \mathrm{p})^{6}$ basis set. The CPCM formalism for the Self Consistent Reaction Field (SCRF) model of solvation was employed in calculations to account for solvation in $\mathrm{MeCN}$, and the default parameters as implemented in Gaussian were used.

Reduction potentials were calculated using a modified procedure as described by Nicewicz and coworkers. ${ }^{7}$ Geometry optimizations were carried out for the reduced and neutral forms of each molecule, and frequency calculations were performed on the minimized structures to ensure no imaginary frequencies existed. Geometry optimizations that did not converge to an energy minimum upon the initial calculation were sequentially optimized using a tight convergence criteria. Gibbs free energies $\left(\mathrm{G}_{298}\right)$ were obtained from the calculation and employed in the following equation:

$$
E_{1 / 2}^{0, \text { calc }}=-\frac{\left(G_{298}[\text { reduced }]-G_{298}[\text { oxidized }]\right)}{n_{e} \mathcal{F}}-E_{1 / 2}^{0, S H E}+E_{1 / 2}^{0, S C E}
$$

Where $n_{e}$ is the number of electrons transferred $\left(n_{e}=1\right.$ for all calculations here), $\mathcal{F}$ is the Faraday constant (value $23.061 \mathrm{kcal} \mathrm{mol}^{-1} \mathrm{~V}^{-1}$ ), $E_{1 / 2}^{0, S H E}$ is the absolute value for the standard hydrogen electrode (SHE, value $=4.281 \mathrm{~V}$ ) and $E_{1 / 2}^{0, S C E}$ is the potential of the saturated calomel electrode $(\mathrm{SCE})$ relative to the $\mathrm{SHE}$ in $\mathrm{MeCN}$ (value $=-0.141 \mathrm{~V})^{8}$, and $\mathrm{G}_{298}$ [oxidized] and $\mathrm{G}_{298}$ [reduced] are the Gibbs free energies in MeCN obtained from DFT calculations.

Steric effects of the arene substituents on cyclization were observed from the minimized geometries of the relevant compounds. The structural minima were visualized using GaussView $5^{9}$ allowing the interatomic distances between the two carbons which form the spirocyclic center to be obtained.

\footnotetext{
${ }^{4}$ M. J. Frisch, G. W. Trucks, H. B. Schlegel, G. E. Scuseria, M. A. Robb, J. R. Cheeseman, G. Scalmani, V. Barone, G. A. Petersson, H. Nakatsuji, X. Li, M. Caricato, A. Marenich, J. Bloino, B. G. Janesko, R. Gomperts, B.

Mennucci, H. P. Hratchian, J. V. Ortiz, A. F. Izmaylov, J. L. Sonnenberg, D. Williams-Young, F. Ding, F. Lipparini, F. Egidi, J. Goings, B. Peng, A. Petrone, T. Henderson, D. Ranasinghe, V. G. Zakrzewski, J. Gao, N. Rega, G. Zheng, W. Liang, M. Hada, M. Ehara, K. Toyota, R. Fukuda, J. Hasegawa, M. Ishida, T. Nakajima, Y. Honda, O. Kitao, H. Nakai, T. Vreven, K. Throssell, J. A. Montgomery, Jr., J. E. Peralta, F. Ogliaro, M. Bearpark, J. J. Heyd, E. Brothers, K. N. Kudin, V. N. Staroverov, T. Keith, R. Kobayashi, J. Normand, K. Raghavachari, A. Rendell, J. C. Burant, S. S. Iyengar, J. Tomasi, M. Cossi, J. M. Millam, M. Klene, C. Adamo, R. Cammi, J. W. Ochterski, R. L. Martin, K. Morokuma, O. Farkas, J. B. Foresman, and D. J. Fox, Gaussian, Inc., Wallingford CT, 2016.

5(a) Lee, C.; Yang, W.; Parr, R. G. Phys. Rev. B, 1988, 37 (2), 785-789. (b) Becke, A. D. J. Chem. Phys. 1993, 98 (7), 5648-5652.

${ }^{6}$ McLean, A. D.; Chandler, G. S. Contracted Gaussian Basis Sets for Molecular Calculations. I. Second Row Atoms, Z=11-18. J. Chem. Phys. 1980, 72, 5639- 5648.

${ }^{7}$ Roth, H. G.; Romero, N. A.; Nicewicz, D. A. Experimental and Calculated Electrochemical Potentials of Common Organic Molecules for Applications to SingleElectron Redox Chemistry. Synlett 2016, 27 (05), $714-723$.

${ }^{8}$ Isse, A. A.; Gennaro, A. Absolute Potential of the Standard Hydrogen Electrode and the Problem of Interconversion of Potentials in Different Solvents. J. Phys. Chem. B 2010, 114 (23), 7894-7899.

${ }^{9}$ Roy Dennington TK and JM. GaussView, Version 5, Semichem Inc, Shawnee Mission KS, 2009.
} 
Molecular coordinates of optimized structures:

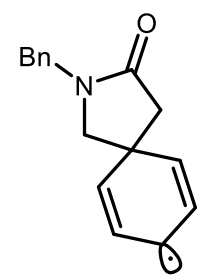

Charge: 0

Multiplicity: 2

Number of imaginary frequencies: 0

Solvation: MeCN

$\mathrm{G}_{298}=-749.250858$ Hartree

$\begin{array}{lrrr}\mathrm{C} & -0.67021525 & 1.67383904 & -3.52647222 \\ \mathrm{C} & 1.62256455 & 1.29631778 & -3.73626915 \\ \mathrm{C} & 0.07672390 & 2.37458623 & -2.38022356 \\ \mathrm{H} & -1.57793076 & 2.15145972 & -3.83115408 \\ \mathrm{H} & -0.90383945 & 0.67001427 & -3.23897339 \\ \mathrm{H} & 1.48810123 & 0.31261662 & -3.33733101 \\ \mathrm{H} & 2.55355683 & 1.31829279 & -4.26321362 \\ \mathrm{O} & -0.45691413 & 2.90442774 & -1.37122951 \\ \mathrm{~N} & 1.53966646 & 2.32415369 & -2.66562245 \\ \mathrm{C} & 1.99332151 & 3.62922748 & -3.16749843 \\ \mathrm{H} & 1.41849308 & 3.89967664 & -4.02850339 \\ \mathrm{H} & 3.02787126 & 3.56890337 & -3.43389629 \\ \mathrm{C} & 1.80692420 & 4.69402465 & -2.07065614 \\ \mathrm{C} & 2.83996131 & 4.94875764 & -1.15858849 \\ \mathrm{C} & 0.60426554 & 5.40825710 & -1.98459730 \\ \mathrm{C} & 2.67034073 & 5.91772490 & -0.16046362 \\ \mathrm{H} & 3.75821850 & 4.40342648 & -1.22429734 \\ \mathrm{C} & 0.43464415 & 6.37722284 & -0.98647109 \\ \mathrm{H} & -0.18448079 & 5.21376361 & -2.68098190 \\ \mathrm{C} & 1.46768203 & 6.63195728 & -0.07440472 \\ \mathrm{H} & 3.45908884 & 6.11222174 & 0.53591803 \\ \mathrm{H} & -0.48361187 & 6.92255620 & -0.92076418 \\ \mathrm{H} & 1.33817319 & 7.37178573 & 0.68768538 \\ \mathrm{C} & 0.41715892 & 1.68130187 & -4.58869847 \\ \mathrm{C} & 0.60945176 & 3.15768691 & -5.12274815 \\ \mathrm{C} & 0.13949927 & 0.65242991 & -5.75754249 \\ \mathrm{C} & 0.94414182 & 3.32317391 & -6.39869152 \\ \mathrm{H} & 0.51126987 & 3.99542129 & -4.46437028 \\ \mathrm{C} & -0.31675236 & 1.12588223 & -6.91301264 \\ \mathrm{H} & 0.27797525 & -0.39785205 & -5.60710243 \\ \mathrm{C} & 0.27516439 & 2.42711245 & -7.42686688 \\ \mathrm{H} & 1.69198010 & 4.03440692 & -6.68115480 \\ \mathrm{H} & -1.10493686 & 0.62654268 & -7.43677329 \\ \mathrm{H} & 0.22350066 & 2.69870937 & -8.46053316\end{array}$




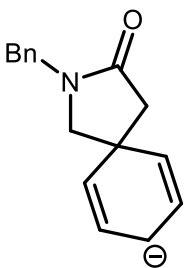

Charge: -1

Multiplicity: 1

Number of imaginary frequencies: 0

Solvation: MeCN

$\mathrm{G}_{298}=-749.361362$ Hartree

$\begin{array}{lrrr}\mathrm{C} & -0.67021525 & 1.67383904 & -3.52647222 \\ \mathrm{C} & 1.62256455 & 1.29631778 & -3.73626915 \\ \mathrm{C} & 0.07672390 & 2.37458623 & -2.38022356 \\ \mathrm{H} & -1.57793076 & 2.15145972 & -3.83115408 \\ \mathrm{H} & -0.90383945 & 0.67001427 & -3.23897339 \\ \mathrm{H} & 1.48810123 & 0.31261662 & -3.33733101 \\ \mathrm{H} & 2.55355683 & 1.31829279 & -4.26321362 \\ \mathrm{O} & -0.45691413 & 2.90442774 & -1.37122951 \\ \mathrm{~N} & 1.53966646 & 2.32415369 & -2.66562245 \\ \mathrm{C} & 1.99332151 & 3.62922748 & -3.16749843 \\ \mathrm{H} & 1.41849308 & 3.89967664 & -4.02850339 \\ \mathrm{H} & 3.02787126 & 3.56890337 & -3.43389629 \\ \mathrm{C} & 1.80692420 & 4.69402465 & -2.07065614 \\ \mathrm{C} & 2.83996131 & 4.94875764 & -1.15858849 \\ \mathrm{C} & 0.60426554 & 5.40825710 & -1.98459730 \\ \mathrm{C} & 2.67034073 & 5.91772490 & -0.16046362 \\ \mathrm{H} & 3.75821850 & 4.40342648 & -1.22429734 \\ \mathrm{C} & 0.43464415 & 6.37722284 & -0.98647109 \\ \mathrm{H} & -0.18448079 & 5.21376361 & -2.68098190 \\ \mathrm{C} & 1.46768203 & 6.63195728 & -0.07440472 \\ \mathrm{H} & 3.45908884 & 6.11222174 & 0.53591803 \\ \mathrm{H} & -0.48361187 & 6.92255620 & -0.92076418 \\ \mathrm{H} & 1.33817319 & 7.37178573 & 0.68768538 \\ \mathrm{C} & 0.41715892 & 1.68130187 & -4.58869847 \\ \mathrm{C} & 0.60945176 & 3.15768691 & -5.12274815 \\ \mathrm{C} & 0.13949927 & 0.65242991 & -5.75754249 \\ \mathrm{C} & 0.94414182 & 3.32317391 & -6.39869152 \\ \mathrm{H} & 0.51126987 & 3.99542129 & -4.46437028 \\ \mathrm{C} & -0.31675236 & 1.12588223 & -6.91301264 \\ \mathrm{H} & 0.27797525 & -0.39785205 & -5.60710243 \\ \mathrm{C} & 0.27516439 & 2.42711245 & -7.42686688 \\ \mathrm{H} & 1.69198010 & 4.03440692 & -6.68115480 \\ \mathrm{H} & -1.10493686 & 0.62654268 & -7.43677329 \\ \mathrm{H} & 0.22350066 & 2.69870937 & -8.46053316\end{array}$


<smiles>CC(C)CN(Cc1ccccc1)C(=O)CCl</smiles>

Charge: 0

Multiplicity: 1

Solvation: MeCN

Number of imaginary frequencies: 0

Interatomic Distance: $3.49 \AA$

$\begin{array}{lrcc}\mathrm{C} & -1.42928837 & 1.11221039 & 0.41076646 \\ \mathrm{O} & -1.33747937 & 1.09112177 & 1.66563575 \\ \mathrm{~N} & -0.21375667 & 1.09002359 & -0.41560538 \\ \mathrm{C} & -2.81505622 & 1.16126145 & -0.25918986 \\ \mathrm{H} & -3.16450854 & 0.16432840 & -0.42921214 \\ \mathrm{H} & -2.74146226 & 1.67760837 & -1.19346486 \\ \mathrm{Cl} & -3.94504256 & 2.00781660 & 0.79155999 \\ \mathrm{C} & 0.84595957 & 0.35633052 & 0.29121565 \\ \mathrm{C} & 0.38046073 & -1.08711028 & 0.55840376 \\ \mathrm{H} & 1.15181881 & -1.62115898 & 1.07289253 \\ \mathrm{H} & 0.17044641 & -1.57212133 & -0.37195008 \\ \mathrm{H} & -0.50431405 & -1.07096071 & 1.15991251 \\ \mathrm{C} & 1.14822317 & 1.05438379 & 1.63022959 \\ \mathrm{H} & 1.91958125 & 0.52033510 & 2.14471837 \\ \mathrm{H} & 0.26344839 & 1.07053337 & 2.23173835 \\ \mathrm{H} & 1.47165419 & 2.05729396 & 1.44458591 \\ \mathrm{C} & 2.11937374 & 0.33308720 & -0.57450723 \\ \mathrm{H} & 2.89073182 & -0.20096150 & -0.06001846 \\ \mathrm{H} & 2.44280476 & 1.33599736 & -0.76015092 \\ \mathrm{H} & 1.90935943 & -0.15192384 & -1.50486107 \\ \mathrm{C} & -0.50228101 & 0.42370000 & -1.69375505 \\ \mathrm{H} & 0.38249377 & 0.40755043 & -2.29526381 \\ \mathrm{H} & -0.82571202 & -0.57921016 & -1.50811136 \\ \mathrm{C} & -1.61245993 & 1.19233084 & -2.43423422 \\ \mathrm{C} & -2.48811378 & 0.50857202 & -3.28841580 \\ \mathrm{C} & -1.74706889 & 2.57554372 & -2.25388869 \\ \mathrm{C} & -3.49837493 & 1.20802651 & -3.96225391 \\ \mathrm{H} & -2.38533832 & -0.54754210 & -3.42611180 \\ \mathrm{C} & -2.75733089 & 3.27499799 & -2.92772574 \\ \mathrm{H} & -1.07848790 & 3.09760871 & -1.60170192 \\ \mathrm{C} & -3.63298329 & 2.59123954 & -3.78190911 \\ \mathrm{H} & -4.16695566 & 0.68596158 & -4.61444100 \\ \mathrm{H} & -2.86010661 & 4.33111204 & -2.79002943 \\ \mathrm{H} & -4.40433884 & 3.12528890 & -4.29640101 \\ & & & \\ & & & \end{array}$




\begin{tabular}{|c|c|c|c|}
\hline \multirow[b]{2}{*}{$\mathrm{C}$} & $\begin{array}{l}\text { Charge } \\
\text { Multip } \\
\text { Numbe } \\
\text { Solvati }\end{array}$ & $\begin{array}{l}0 \\
\text { licity: } 1 \\
\text { r of imaginary } \\
\text { on: } \mathrm{MeCN}\end{array}$ & $\begin{array}{l}\text { frequencies: } 0 \\
3.42 \AA\end{array}$ \\
\hline & -0.43282831 & -0.79332914 & 0.01121010 \\
\hline $\mathrm{O}$ & -1.15350877 & -1.79569780 & -0.23260806 \\
\hline $\mathrm{N}$ & 1.03169377 & -0.91818165 & -0.01085634 \\
\hline $\mathrm{C}$ & -1.08513793 & 0.56414367 & 0.33270619 \\
\hline $\mathrm{H}$ & -1.23097314 & 1.11340606 & -0.57390449 \\
\hline $\mathrm{H}$ & -0.44744177 & 1.12003940 & 0.98785774 \\
\hline $\mathrm{Cl}$ & -2.63967693 & 0.29771101 & 1.11374532 \\
\hline $\mathrm{C}$ & 1.42013417 & -1.96127084 & -0.97105371 \\
\hline $\mathrm{C}$ & 2.95439540 & -2.09206871 & -0.99417094 \\
\hline $\mathrm{H}$ & 3.23713774 & -2.85132411 & -1.69309011 \\
\hline $\mathrm{H}$ & 3.30449213 & -2.35723106 & -0.01845520 \\
\hline $\mathrm{H}$ & 3.38756900 & -1.15853001 & -1.28702948 \\
\hline $\mathrm{C}$ & 0.91625664 & -1.57963532 & -2.37535488 \\
\hline $\mathrm{H}$ & 1.19899898 & -2.33889072 & -3.07427405 \\
\hline $\mathrm{H}$ & 1.34943024 & -0.64609662 & -2.66821341 \\
\hline $\mathrm{H}$ & -0.14975603 & -1.48875628 & -2.35929291 \\
\hline $\mathrm{C}$ & 0.79668805 & -3.30486860 & -0.54955638 \\
\hline $\mathrm{H}$ & 1.07943039 & -4.06412400 & -1.24847556 \\
\hline $\mathrm{H}$ & -0.26932462 & -3.21398956 & -0.53349441 \\
\hline $\mathrm{H}$ & 1.14678478 & -3.57003094 & 0.42615936 \\
\hline $\mathrm{C}$ & 1.62680143 & 0.36434348 & -0.41319471 \\
\hline $\mathrm{H}$ & 1.27670470 & 0.62950582 & -1.38891045 \\
\hline $\mathrm{H}$ & 2.69281410 & 0.27346444 & -0.42925668 \\
\hline $\mathrm{C}$ & 1.21986386 & 1.45710358 & 0.59272635 \\
\hline $\mathrm{C}$ & 1.09241111 & 2.78690766 & 0.16928938 \\
\hline $\mathrm{C}$ & 0.97700342 & 1.12171120 & 1.93155147 \\
\hline $\mathrm{C}$ & 0.72209430 & 3.78131876 & 1.08467673 \\
\hline $\mathrm{C}$ & 0.60668597 & 2.11612218 & 2.84693868 \\
\hline $\mathrm{H}$ & 1.07431902 & 0.10637671 & 2.25485557 \\
\hline $\mathrm{C}$ & 0.47923215 & 3.44592608 & 2.42350147 \\
\hline $\mathrm{H}$ & 0.62478130 & 4.79665368 & 0.76137321 \\
\hline $\mathrm{H}$ & 0.42125398 & 1.86004221 & 3.86916078 \\
\hline $\mathrm{H}$ & 0.19648575 & 4.20518080 & 3.12241974 \\
\hline $\mathrm{C}$ & 1.35929469 & 3.15547139 & -1.30194613 \\
\hline $\mathrm{H}$ & 1.72782650 & 4.15848454 & -1.35716320 \\
\hline $\mathrm{H}$ & 0.44934096 & 3.07862445 & -1.85960082 \\
\hline $\mathrm{H}$ & 2.08614872 & 2.48538517 & -1.71129646 \\
\hline
\end{tabular}




\begin{tabular}{|c|c|c|c|}
\hline \multirow[b]{2}{*}{$\mathrm{C}$} & $\begin{array}{l}\text { Charge } \\
\text { Multip } \\
\text { Numbe } \\
\text { Solvat }\end{array}$ & $\begin{array}{l}0 \\
\text { licity: } 1 \\
\text { of of imaginary } \\
\text { on: MeCN } \\
\text { omic Distance: }\end{array}$ & $\begin{array}{l}\text { frequencies: } 0 \\
2.91 \AA\end{array}$ \\
\hline & -0.46509528 & 0.55862783 & $2.91 \AA$ \\
\hline $\mathrm{O}$ & -1.11792889 & -0.50351715 & 0.00548049 \\
\hline $\mathrm{N}$ & 0.99291720 & 0.56343212 & -0.01084802 \\
\hline $\mathrm{C}$ & -1.19361469 & 1.85342254 & 0.58186015 \\
\hline $\mathrm{H}$ & -1.48812414 & 2.38725389 & -0.29745111 \\
\hline $\mathrm{H}$ & -0.53748551 & 2.46370861 & 1.16662114 \\
\hline $\mathrm{Cl}$ & -2.62102207 & 1.45127518 & 1.52969224 \\
\hline $\mathrm{C}$ & 1.36083931 & -0.44177984 & -1.01836092 \\
\hline $\mathrm{C}$ & 0.67065848 & -0.10673774 & -2.35365012 \\
\hline $\mathrm{H}$ & 0.93846576 & -0.83842258 & -3.08700986 \\
\hline $\mathrm{H}$ & 0.98458438 & 0.86123309 & -2.68438097 \\
\hline $\mathrm{H}$ & -0.39061595 & -0.11023476 & -2.21732540 \\
\hline $\mathrm{C}$ & 0.90902068 & -1.83493418 & -0.54235572 \\
\hline $\mathrm{H}$ & 1.17682788 & -2.56661908 & -1.27571541 \\
\hline $\mathrm{H}$ & -0.15225374 & -1.83843116 & -0.40603096 \\
\hline $\mathrm{H}$ & 1.38856193 & -2.06772318 & 0.38541010 \\
\hline $\mathrm{C}$ & 2.88828097 & -0.43674675 & -1.21456659 \\
\hline $\mathrm{H}$ & 3.15608824 & -1.16843160 & -1.94792629 \\
\hline $\mathrm{H}$ & 3.36782219 & -0.66953570 & -0.28680076 \\
\hline $\mathrm{H}$ & 3.20220688 & 0.53122407 & -1.54529744 \\
\hline $\mathrm{C}$ & 1.42419860 & 1.89326126 & -0.46521659 \\
\hline $\mathrm{H}$ & 0.94465737 & 2.12605024 & -1.39298246 \\
\hline $\mathrm{H}$ & 2.48547299 & 1.89675826 & -0.60154133 \\
\hline $\mathrm{C}$ & 1.03875641 & 2.94634046 & 0.59027312 \\
\hline $\mathrm{C}$ & 0.74834651 & 4.25951975 & 0.19638959 \\
\hline $\mathrm{C}$ & 0.97841390 & 2.59146326 & 1.94465228 \\
\hline $\mathrm{C}$ & 0.39759530 & 5.21782213 & 1.15688535 \\
\hline $\mathrm{C}$ & 0.62766535 & 3.54976632 & 2.90514834 \\
\hline $\mathrm{C}$ & 0.33725629 & 4.86294581 & 2.51126490 \\
\hline $\mathrm{H}$ & 0.17586062 & 6.22046358 & 0.85614650 \\
\hline $\mathrm{H}$ & 0.58159357 & 3.27880998 & 3.93924700 \\
\hline $\mathrm{H}$ & 0.06944982 & 5.59463092 & 3.24462471 \\
\hline $\mathrm{C}$ & 0.81465566 & 4.64949437 & -1.29193930 \\
\hline $\mathrm{H}$ & 1.08524402 & 5.68107847 & -1.37863557 \\
\hline $\mathrm{H}$ & -0.14199254 & 4.49242142 & -1.74476641 \\
\hline $\mathrm{H}$ & 1.54678741 & 4.04593963 & -1.78651457 \\
\hline $\mathrm{C}$ & 1.29754143 & 1.14840803 & 2.37749085 \\
\hline $\mathrm{H}$ & 1.70949193 & 1.15507812 & 3.36498888 \\
\hline $\mathrm{H}$ & 2.00541810 & 0.72069865 & 1.69861295 \\
\hline $\mathrm{H}$ & 0.39944568 & 0.56680505 & 2.3696092 \\
\hline
\end{tabular}


<smiles>CC(C)CN(Cc1cccc2[nH]ccc12)C(=O)CCl</smiles>

Charge: 0

Multiplicity: 1

Number of imaginary frequencies: 0

Solvation: MeCN

Interatomic Distance: $3.41 \AA$

$\begin{array}{lrrr}\mathrm{C} & -0.50779625 & 1.50532687 & 0.33496392 \\ \mathrm{O} & -1.15791776 & 0.45474692 & 0.09581049 \\ \mathrm{~N} & 0.95129954 & 1.44454317 & 0.50302599 \\ \mathrm{C} & -1.24076953 & 2.85467982 & 0.45156918 \\ \mathrm{H} & -1.29019019 & 3.31812571 & -0.51158995 \\ \mathrm{H} & -0.70944740 & 3.49197138 & 1.12718464 \\ \mathrm{Cl} & -2.87111364 & 2.58623869 & 1.05780137 \\ \mathrm{C} & 1.49214433 & 0.34225853 & -0.30527808 \\ \mathrm{C} & 1.15581445 & 0.58071586 & -1.78906391 \\ \mathrm{H} & 1.54949055 & -0.22162741 & -2.37742131 \\ \mathrm{H} & 1.59051752 & 1.50449655 & -2.10931771 \\ \mathrm{H} & 0.09375155 & 0.62495983 & -1.91139483 \\ \mathrm{C} & 0.86649699 & -0.98729501 & 0.15564797 \\ \mathrm{H} & 1.26017309 & -1.78963827 & -0.43270941 \\ \mathrm{H} & -0.19556591 & -0.94305104 & 0.03331709 \\ \mathrm{H} & 1.10018077 & -1.15297640 & 1.18659006 \\ \mathrm{C} & 3.02072084 & 0.27858039 & -0.12921309 \\ \mathrm{H} & 3.41439695 & -0.52376286 & -0.71757049 \\ \mathrm{H} & 3.25440467 & 0.11289902 & 0.90172898 \\ \mathrm{H} & 3.45542381 & 1.20236111 & -0.44946690 \\ \mathrm{C} & 1.54850833 & 2.71366245 & 0.06305114 \\ \mathrm{H} & 1.31482454 & 2.87934380 & -0.96789094 \\ \mathrm{H} & 2.61057125 & 2.66941854 & 0.18538202 \\ \mathrm{C} & 0.98190907 & 3.86843687 & 0.90984587 \\ \mathrm{C} & 0.85691106 & 5.15148359 & 0.38937984 \\ \mathrm{C} & 0.57797504 & 3.66702136 & 2.24537631 \\ \mathrm{C} & 0.28995697 & 6.17897067 & 1.08982434 \\ \mathrm{C} & 0.07149149 & 4.75199263 & 3.00918067 \\ \mathrm{H} & 0.65659470 & 2.69506976 & 2.68586357 \\ \mathrm{C} & -0.07451198 & 6.02921740 & 2.42534016 \\ \mathrm{H} & -0.20387837 & 4.59989838 & 4.03189215 \\ \mathrm{H} & -0.45919004 & 6.85348853 & 2.98881146 \\ \mathrm{C} & 1.30281089 & 5.70566587 & -0.95218599 \\ \mathrm{H} & 1.77466013 & 5.16100102 & -1.74313384 \\ \mathrm{C} & 1.00986056 & 7.03255115 & -0.94292059 \\ \mathrm{H} & 1.35306981 & 7.73623853 & -1.67225692 \\ \mathrm{~N} & 0.14972544 & 7.37497755 & 0.22779087 \\ \mathrm{H} & -0.80086996 & 7.48509673 & -0.06245413\end{array}$




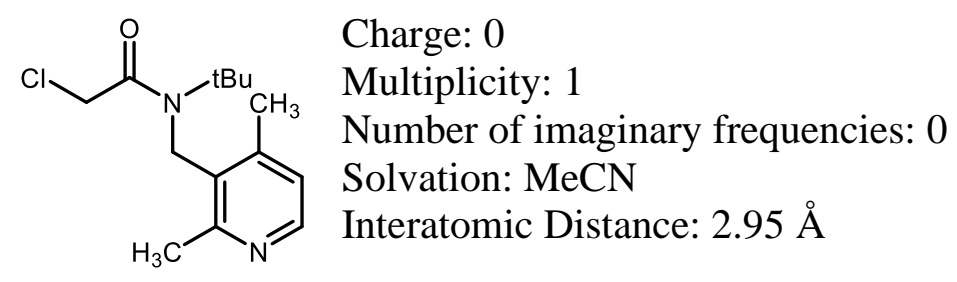

$\begin{array}{lrrr}\mathrm{C} & 2.18821795 & 1.15575564 & -1.92603099 \\ \mathrm{O} & 2.78368334 & 0.66024971 & -2.91772923 \\ \mathrm{~N} & 2.95555049 & 1.83421108 & -0.87161220 \\ \mathrm{C} & 0.65562940 & 1.05138115 & -1.81704346 \\ \mathrm{H} & 0.20600082 & 1.90176989 & -2.28562977 \\ \mathrm{H} & 0.37263454 & 1.02133901 & -0.78558266 \\ \mathrm{Cl} & 0.10916246 & -0.41725914 & -2.61833592 \\ \mathrm{C} & 2.12875659 & 2.88700819 & -0.26421602 \\ \mathrm{H} & 2.68729115 & 3.38084986 & 0.50328614 \\ \mathrm{H} & 1.84790004 & 3.59695636 & -1.01387807 \\ \mathrm{C} & 4.16382720 & 2.43521874 & -1.45449914 \\ \mathrm{C} & 5.02999225 & 1.33228845 & -2.09081892 \\ \mathrm{H} & 5.90948618 & 1.76975660 & -2.51509717 \\ \mathrm{H} & 4.47145770 & 0.83844672 & -2.85832104 \\ \mathrm{H} & 5.31084886 & 0.62234032 & -1.34115685 \\ \mathrm{C} & 3.75960369 & 3.45701324 & -2.53345204 \\ \mathrm{H} & 4.63909764 & 3.89448139 & -2.95773029 \\ \mathrm{H} & 3.15778772 & 4.22333495 & -2.09133377 \\ \mathrm{H} & 3.20106914 & 2.96317151 & -3.30095416 \\ \mathrm{C} & 4.96769935 & 3.14598163 & -0.34986994 \\ \mathrm{H} & 5.84719328 & 3.58344980 & -0.77414818 \\ \mathrm{H} & 5.24855595 & 2.43603351 & 0.39979215 \\ \mathrm{H} & 4.36588336 & 3.91230333 & 0.09224833 \\ \mathrm{C} & 0.86294285 & 2.25738114 & 0.34642737 \\ \mathrm{C} & -0.32966103 & 2.99049776 & 0.41391645 \\ \mathrm{C} & 0.89268568 & 0.95148609 & 0.83775696 \\ \mathrm{C} & -0.27219432 & 0.41145146 & 1.38462852 \\ \mathrm{C} & -1.43705684 & 1.18968051 & 1.42674257 \\ \mathrm{H} & -0.27597075 & -0.58743090 & 1.76819045 \\ \mathrm{H} & -2.33361790 & 0.78175801 & 1.84468694 \\ \mathrm{~N} & -1.43592273 & 2.44433269 & 0.94602106 \\ \mathrm{C} & -0.37241114 & 4.43225234 & -0.12563537 \\ \mathrm{H} & -1.09216047 & 4.99884195 & 0.42739004 \\ \mathrm{H} & -0.64768225 & 4.41814592 & -1.15952455 \\ \mathrm{H} & 0.59290631 & 4.88150770 & -0.01965502 \\ \mathrm{C} & 2.18814983 & 0.12097726 & 0.77752642 \\ \mathrm{H} & 2.20814867 & -0.56851182 & 1.59551472 \\ \mathrm{H} & 2.22281108 & -0.41938888 & -0.14535116\end{array}$


<smiles>CC(C)CN(Cc1ccccc1Cl)C(=O)CCl</smiles>

Charge: 0

Multiplicity: 1

Number of imaginary frequencies: 0

Solvation: MeCN

Interatomic Distance: $3.42 \AA$

$\begin{array}{lrrr}\mathrm{C} & -0.99633452 & 0.18519537 & -0.16020934 \\ \mathrm{O} & -1.62020267 & -0.87186988 & -0.43764791 \\ \mathrm{~N} & 0.46878452 & 0.16454722 & -0.04231174 \\ \mathrm{C} & -1.76774614 & 1.50043808 & 0.05580161 \\ \mathrm{H} & -1.86432111 & 2.01469876 & -0.87753071 \\ \mathrm{H} & -1.23465676 & 2.11684354 & 0.74917022 \\ \mathrm{Cl} & -3.36736441 & 1.14378531 & 0.69737794 \\ \mathrm{C} & 1.01711452 & -0.87224924 & -0.92847612 \\ \mathrm{C} & 0.62362710 & -0.56330213 & -2.38495109 \\ \mathrm{H} & 1.02275166 & -1.31797710 & -3.02998230 \\ \mathrm{H} & 1.01755263 & 0.39100131 & -2.66606963 \\ \mathrm{H} & -0.44282009 & -0.54827252 & -2.47076771 \\ \mathrm{C} & 0.45015628 & -2.24573269 & -0.52387561 \\ \mathrm{H} & 0.84928084 & -3.00040766 & -1.16890682 \\ \mathrm{H} & -0.61629092 & -2.23070308 & -0.60969223 \\ \mathrm{H} & 0.72355338 & -2.46039075 & 0.48809077 \\ \mathrm{C} & 2.55200114 & -0.89388064 & -0.80496435 \\ \mathrm{H} & 2.95112570 & -1.64855561 & -1.44999557 \\ \mathrm{H} & 2.82539824 & -1.10853870 & 0.20700202 \\ \mathrm{H} & 2.94592667 & 0.06042280 & -1.08608289 \\ \mathrm{C} & 1.00997194 & 1.47559960 & -0.42852132 \\ \mathrm{H} & 0.73657483 & 1.69025766 & -1.44048770 \\ \mathrm{H} & 2.07641913 & 1.46056999 & -0.34270470 \\ \mathrm{C} & 0.43553098 & 2.56176732 & 0.49984136 \\ \mathrm{C} & 0.25787743 & 3.86791526 & 0.02409065 \\ \mathrm{C} & 0.09044326 & 2.24403201 & 1.82040211 \\ \mathrm{C} & -0.26486135 & 4.85632852 & 0.86890149 \\ \mathrm{C} & -0.43229680 & 3.23244494 & 2.66521254 \\ \mathrm{H} & 0.22608547 & 1.24675902 & 2.18364826 \\ \mathrm{C} & -0.60994818 & 4.53859343 & 2.18946253 \\ \mathrm{H} & -0.40050316 & 5.85360161 & 0.50565546 \\ \mathrm{H} & -0.69577712 & 2.98984744 & 3.67349056 \\ \mathrm{H} & -1.00906898 & 5.29326936 & 2.83449495 \\ \mathrm{Cl} & 0.69126501 & 4.26695399 & -1.63438555\end{array}$


<smiles>CC(C)CN(Cc1ccccc1F)C(=O)CCl</smiles>

Charge: 0

Multiplicity: 1

Number of imaginary frequencies: 0

Solvation: MeCN

Interatomic Distance: $3.43 \AA$

$\begin{array}{lrcc}\mathrm{C} & -0.61568894 & 0.26849260 & 0.52217769 \\ \mathrm{O} & -1.18280256 & -0.82303094 & 0.78775079 \\ \mathrm{~N} & 0.85162121 & 0.35651459 & 0.53454962 \\ \mathrm{C} & 1.42378791 & -0.94638530 & 0.16579882 \\ \mathrm{C} & 0.94481915 & -1.33665208 & -1.24483252 \\ \mathrm{H} & 1.36129423 & -2.28502138 & -1.51324297 \\ \mathrm{H} & 1.26359618 & -0.59537172 & -1.94753064 \\ \mathrm{H} & -0.12322293 & -1.40072250 & -1.25383794 \\ \mathrm{C} & 0.96498733 & -2.01327478 & 1.17715873 \\ \mathrm{H} & 1.38146241 & -2.96164409 & 0.90874829 \\ \mathrm{H} & -0.10305474 & -2.07734521 & 1.16815331 \\ \mathrm{H} & 1.29777731 & -1.74211540 & 2.15727271 \\ \mathrm{C} & 2.96096997 & -0.85417179 & 0.17875989 \\ \mathrm{H} & 3.37744504 & -1.80254109 & -0.08965056 \\ \mathrm{H} & 3.29375994 & -0.58301240 & 1.15887387 \\ \mathrm{H} & 3.27974699 & -0.11289143 & -0.52393824 \\ \mathrm{C} & 1.28956721 & 1.37490909 & -0.43083939 \\ \mathrm{H} & 0.95677723 & 1.10374971 & -1.41095337 \\ \mathrm{H} & 2.35760929 & 1.43897952 & -0.42183398 \\ \mathrm{C} & -1.45885082 & 1.51205963 & 0.18421457 \\ \mathrm{H} & -1.63090453 & 1.55026933 & -0.87117045 \\ \mathrm{H} & -0.93494532 & 2.39269024 & 0.49229369 \\ \mathrm{Cl} & -3.00121129 & 1.42191558 & 1.02718446 \\ \mathrm{C} & 0.69015448 & 2.73985183 & -0.04452903 \\ \mathrm{C} & 0.40768852 & 3.68743915 & -1.03761171 \\ \mathrm{C} & 0.42715486 & 3.03436241 & 1.30009608 \\ \mathrm{C} & -0.13777457 & 4.92953807 & -0.68606902 \\ \mathrm{C} & -0.11830950 & 4.27646080 & 1.65163863 \\ \mathrm{H} & 0.64282365 & 2.31085836 & 2.05833672 \\ \mathrm{C} & -0.40077329 & 5.22404901 & 0.65855618 \\ \mathrm{H} & -0.35344298 & 5.65304227 & -1.44430962 \\ \mathrm{H} & -0.31911375 & 4.50132690 & 2.67828993 \\ \mathrm{H} & -0.81724459 & 6.17241986 & 0.92696703 \\ \mathrm{~F} & 0.66103916 & 3.40372938 & -2.33291948\end{array}$


<smiles>COc1ccc(F)c(CN(CC(C)C)C(=O)CCl)c1</smiles>

Charge: 0

Multiplicity: 1

Number of imaginary frequencies: 0

Solvation: MeCN

Interatomic Distance: $3.43 \AA$

$\begin{array}{lccc}\mathrm{C} & -0.53706787 & 0.37443739 & 0.68435628 \\ \mathrm{O} & -1.11307434 & -0.69793748 & 1.00341623 \\ \mathrm{~N} & 0.92925254 & 0.42180201 & 0.59183032 \\ \mathrm{C} & 1.43849310 & -0.90045013 & 0.20039280 \\ \mathrm{C} & 0.85021447 & -1.29270053 & -1.16769633 \\ \mathrm{H} & 1.22088617 & -2.25515616 & -1.45262024 \\ \mathrm{H} & 1.13812537 & -0.56830622 & -1.90067668 \\ \mathrm{H} & -0.21710719 & -1.32717681 & -1.10034750 \\ \mathrm{C} & 1.02411667 & -1.94303633 & 1.25533649 \\ \mathrm{H} & 1.39478838 & -2.90549196 & 0.97041258 \\ \mathrm{H} & -0.04320499 & -1.97751261 & 1.32268532 \\ \mathrm{H} & 1.43285572 & -1.67049871 & 2.20589192 \\ \mathrm{C} & 2.97463829 & -0.85083005 & 0.10346084 \\ \mathrm{H} & 3.34531000 & -1.81328569 & -0.18146307 \\ \mathrm{H} & 3.38337735 & -0.57829243 & 1.05401628 \\ \mathrm{H} & 3.26254919 & -0.12643574 & -0.62951951 \\ \mathrm{C} & 1.32479368 & 1.41699793 & -0.41516138 \\ \mathrm{H} & 0.91605462 & 1.14446031 & -1.36571682 \\ \mathrm{H} & 2.39211534 & 1.45147421 & -0.48251022 \\ \mathrm{C} & -1.36831004 & 1.63716418 & 0.39083027 \\ \mathrm{H} & -1.61379233 & 1.66856837 & -0.65015591 \\ \mathrm{H} & -0.80032440 & 2.50622755 & 0.64971473 \\ \mathrm{Cl} & -2.84877388 & 1.59913760 & 1.34181965 \\ \mathrm{C} & 0.79130357 & 2.80221445 & -0.00508398 \\ \mathrm{C} & 0.46464029 & 3.74634028 & -0.98785532 \\ \mathrm{C} & 0.63249084 & 3.11863567 & 1.35085779 \\ \mathrm{C} & -0.02083759 & 5.00688661 & -0.61468493 \\ \mathrm{C} & 0.14701590 & 4.37918311 & 1.72402826 \\ \mathrm{C} & -0.17964861 & 5.32330846 & 0.74125689 \\ \mathrm{H} & -0.27025496 & 5.72774660 & -1.36505274 \\ \mathrm{H} & -0.55031993 & 6.28576425 & 1.02618081 \\ \mathrm{~F} & 0.61762651 & 3.44152402 & -2.29406443 \\ \mathrm{H} & 0.88190600 & 2.39777484 & 2.10122553 \\ \mathrm{O} & -0.01503554 & 4.70206279 & 3.10764239 \\ \mathrm{C} & 0.17093435 & 6.10760855 & 3.29407287 \\ \mathrm{H} & 0.04967908 & 6.34920383 & 4.32936456 \\ \mathrm{H} & -0.55284110 & 6.64295259 & 2.71575130 \\ \mathrm{H} & 1.15511737 & 6.38237129 & 2.97659970\end{array}$


A screw-top test tube was charged with 3DPAFIPN (5 mol\%) and the substrate (1.0 equiv). The tube was equipped with a stir bar and sealed with a PTFE/silicon septa. The atmosphere was exchanged by applying vacuum and backfilling with nitrogen (this process was conducted a total of three times). Under nitrogen atmosphere, DIPEA (3 equiv) was added via syringe, followed by degassed solvent. The resulting mixture was stirred at $50{ }^{\circ} \mathrm{C}$ for $16 \mathrm{~h}$ under irradiation by blue LEDs. The reaction was then extracted with ethyl acetate $(3 \mathrm{x})$ and concentrated under reduced pressure. $\mathrm{CDCl}_{3}$ and an internal standard of dibromomethane (1 equiv) were added. The sample was analyzed by ${ }^{1} \mathrm{H}$ NMR $(\mathrm{d} 1=5 \mathrm{~s})$, and the integral values were used to calculate the ratio of dearomatized product to hydrodehalogenation

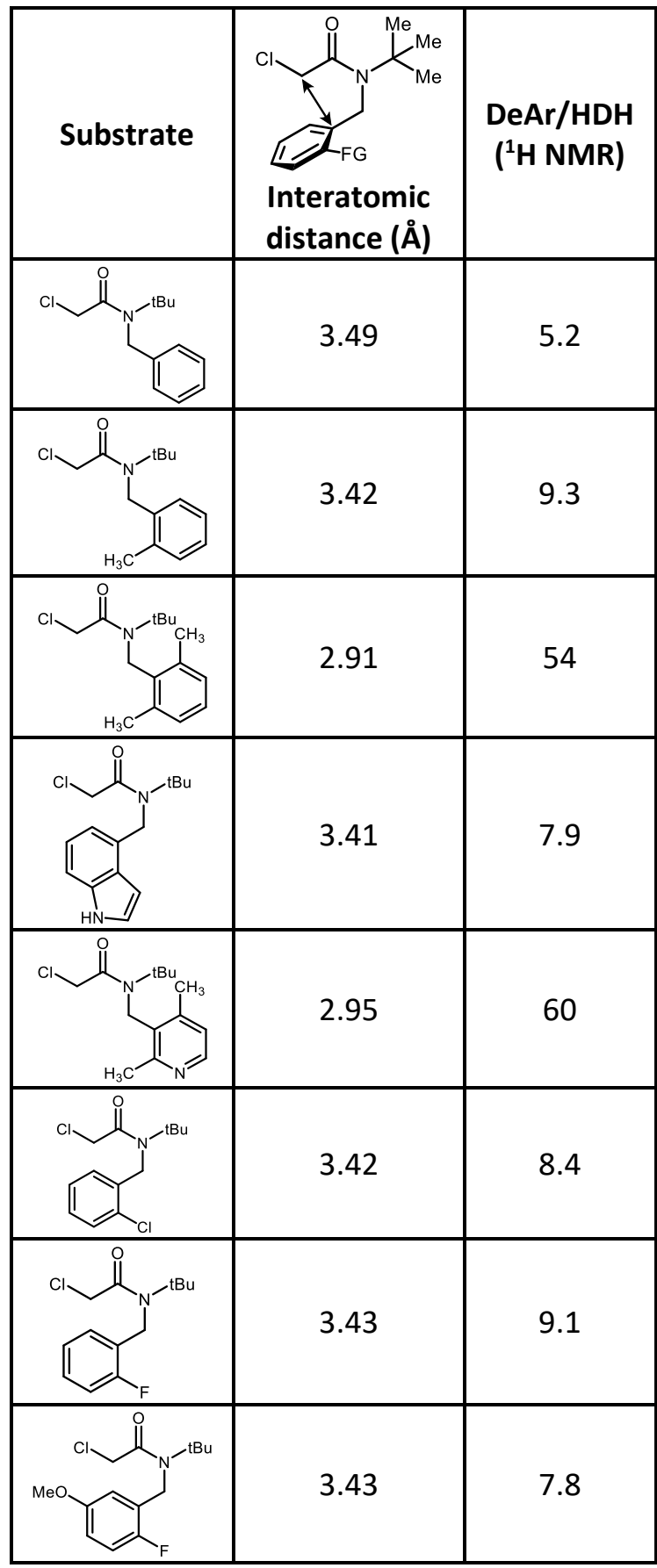

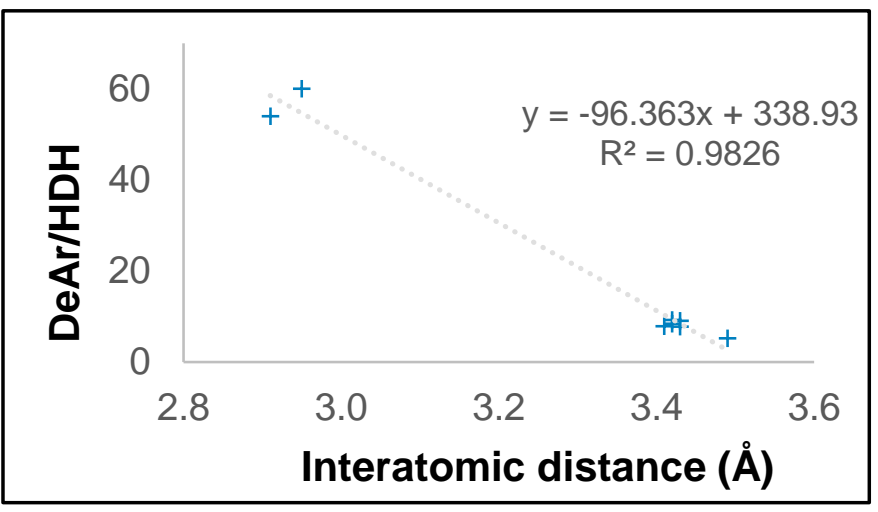




\section{Preparation of Starting Materials}<smiles>CC(C)(C)NCc1cccc2[nH]ccc12</smiles>

\section{$N$-((1H-indol-4-yl)methyl)-2-methylpropan-2-amine (S1):}

Prepared according to General Reductive Amination Procedure. Indole-4-carboxaldehyde (435 $\mathrm{mg}, 3.0 \mathrm{mmol}, 1.0$ equiv) and tert-butyl amine $(0.38 \mathrm{~mL}, 3.6 \mathrm{mmol}, 1.2$ equiv) were stirred in $\mathrm{MeOH}(15 \mathrm{~mL}, 0.2 \mathrm{M})$ for 16 hours. After $\mathrm{NaBH}_{4}(170 \mathrm{mg}, 4.5 \mathrm{mmol}, 1.5$ equiv) was added, the reaction was stirred for an additional $30 \mathrm{~min}$. The title compound was obtained as a light-brown amorphous solid (607 mg, 100\%).

${ }^{1} \mathbf{H}$ NMR $\left(400 \mathrm{MHz}, \mathrm{CDCl}_{3}\right) \delta 8.24(\mathrm{br} \mathrm{s}, 1 \mathrm{H}), 7.29(\mathrm{~d}, \mathrm{~J}=7.8 \mathrm{~Hz}, 1 \mathrm{fH}), 7.24-7.17(\mathrm{~m}, 1 \mathrm{H})$, $7.18-7.08(\mathrm{~m}, 2 \mathrm{H}), 6.65$ (ddd, J = 3.2, 2.1, $1.0 \mathrm{~Hz}, 1 \mathrm{H}), 4.04(\mathrm{~s}, 2 \mathrm{H}), 1.25$ (s, 9H) ppm.

${ }^{13}$ C NMR $\left(151 \mathrm{MHz}, \mathrm{CDCl}_{3}\right) \delta 136.1,133.3,127.1,124.0,122.4,119.40$ 110.0, 100.9, 51.0, 45.3, $29.3 \mathrm{ppm}$.

LRMS (EI) m/z: [M] calc'd. for $\mathrm{C}_{13} \mathrm{H}_{18} \mathrm{~N}_{2}, 202.2$, found 202.2.<smiles>CC(C)(C)N(Cc1cccc2[nH]ccc12)C(=O)CCl</smiles>

\section{$N$-((1H-indol-4-yl)methyl)- $N$-(tert-butyl)-2-chloroacetamide (S2):}

Prepared according to General Acylation Procedure. $N$-((1H-indol-4-yl)methyl)-2-methylpropan2-amine (S1) (474 mg, $2.3 \mathrm{mmol}, 1.0$ equiv), $\mathrm{Et}_{3} \mathrm{~N}$ (0.39 mL, $2.8 \mathrm{mmol}, 1.2 \mathrm{equiv}$ ), and chloroacetyl chloride $\left(0.19 \mathrm{~mL}, 2.3 \mathrm{mmol}, 1.0\right.$ equiv) were stirred in $\mathrm{CH}_{2} \mathrm{Cl}_{2}(23 \mathrm{~mL}, 0.1 \mathrm{M})$ for 30 minutes. Purification on silica gel (30\% EtOAc/hexanes) afforded the title compound as a lightbrown amorphous solid (535 $\mathrm{mg}, 83 \%$ ).

${ }^{1} \mathbf{H}$ NMR (400 MHz, $\left.\mathrm{CDCl}_{3}\right) \delta 8.41(\mathrm{br} \mathrm{s}, 1 \mathrm{H}), 7.36(\mathrm{dt}, \mathrm{J}=8.2,0.9 \mathrm{~Hz}, 1 \mathrm{H}), 7.29$ (dd, J = 3.3, 2.4 $\mathrm{Hz}, 1 \mathrm{H}), 7.21(\mathrm{dd}, \mathrm{J}=8.2,7.3 \mathrm{~Hz}, 1 \mathrm{H}), 6.98(\mathrm{dq}, \mathrm{J}=7.2,1.0 \mathrm{~Hz}, 1 \mathrm{H}), 6.56$ (ddd, J = 3.1, 2.0, 1.0 $\mathrm{Hz}, 1 \mathrm{H}), 4.92$ (s, 2H), 4.01 (s, 2H), $1.51(\mathrm{~s}, 9 \mathrm{H}) \mathrm{ppm}$.

${ }^{13}$ C NMR $\left(151 \mathrm{MHz}, \mathrm{CDCl}_{3}\right) \delta 168.0,135.9,130.4,124.7,124.7,122.6,116.1,110.6,99.9,58.8$, 46.9, 44.5, $28.5 \mathrm{ppm}$.

LRMS (EI) m/z: [M] ${ }^{+}$calc'd. for $\mathrm{C}_{15} \mathrm{H}_{19} \mathrm{ClN}_{2} \mathrm{O}$, 278.1, found 278.1.<smiles>CC(C)(C)NCc1c[nH]c2ccccc12</smiles>

\section{$\mathrm{N}$-((1H-indol-3-yl)methyl)-2-methylpropan-2-amine (S3):}

Prepared according to General Reductive Amination Procedure. Indole-3-carbaldehyde (871 mg, $6.0 \mathrm{mmol}, 1.0$ equiv) and tert-butyl amine $(0.76 \mathrm{~mL}, 7.2 \mathrm{mmol}, 1.2$ equiv) were stirred in $\mathrm{MeOH}$ (30 mL, $0.2 \mathrm{M}$ ) for 16 hours. After $\mathrm{NaBH}_{4}(340 \mathrm{mg}, 9.0 \mathrm{mmol}, 1.5$ equiv) was added, the reaction was stirred for an additional 4 hours. The title compound was obtained as an off-white amorphous solid (627 mg, 52\%). 
${ }^{1} \mathbf{H}$ NMR $\left(600 \mathrm{MHz}, \mathrm{CDCl}_{3}\right) \delta 8.11(\mathrm{br} \mathrm{s}, 1 \mathrm{H}), 7.65(\mathrm{~d}, \mathrm{~J}=7.9 \mathrm{~Hz}, 1 \mathrm{H}), 7.35(\mathrm{~d}, \mathrm{~J}=8.1 \mathrm{~Hz}, 1 \mathrm{H})$, $7.20(\mathrm{t}, \mathrm{J}=7.6 \mathrm{~Hz}, 1 \mathrm{H}), 7.16(\mathrm{~d}, \mathrm{~J}=2.3 \mathrm{~Hz}, 1 \mathrm{H}), 7.13(\mathrm{t}, \mathrm{J}=7.5 \mathrm{~Hz}, 1 \mathrm{H}), 3.96(\mathrm{~s}, 2 \mathrm{H}), 1.25(\mathrm{~s}$, 9H) $\mathrm{ppm}$.

${ }^{13}$ C NMR $\left(151 \mathrm{MHz}, \mathrm{CDCl}_{3}\right) \delta 136.6,127.2,122.5,122.2,119.6,118.9,115.9,111.4,50.8,38.1$, $29.2 \mathrm{ppm}$.

LRMS (APCI) m/z: [M+1] $]^{+}$calc'd. for $\mathrm{C}_{13} \mathrm{H}_{18} \mathrm{~N}_{2}, 203.2$, found 203.2.<smiles>CN(Cc1c[nH]c2ccccc12)C(=O)CCl</smiles>

$N$-((1H-indol-3-yl)methyl)- $N$-(tert-butyl)-2-chloroacetamide (S4):

Prepared according to General Acylation Procedure. $N$-((1H-indol-3-yl)methyl)-2-methylpropan2-amine (S3) $\left(506 \mathrm{mg}, 2.5 \mathrm{mmol}, 1.0\right.$ equiv), $\mathrm{Et}_{3} \mathrm{~N}$ (0.35 mL, $2.5 \mathrm{mmol}, 1.0$ equiv), and chloroacetyl chloride $\left(0.20 \mathrm{~mL}, 2.5 \mathrm{mmol}, 1.0\right.$ equiv) were stirred in $\mathrm{CH}_{2} \mathrm{Cl}_{2}(25 \mathrm{~mL}, 0.1 \mathrm{M})$ for 18 hours. Purification on silica gel (30\% EtOAc/hexanes) afforded the title compound as an offwhite amorphous solid (534 mg, 77\%).

${ }^{1} \mathbf{H}$ NMR $\left(600 \mathrm{MHz}, \mathrm{C}_{6} \mathrm{D}_{6}\right) \delta 7.55(\mathrm{~s}, 1 \mathrm{H}), 7.41(\mathrm{~d}, \mathrm{~J}=7.8 \mathrm{~Hz}, 1 \mathrm{H}), 7.24(\mathrm{t}, \mathrm{J}=7.6 \mathrm{~Hz}, 1 \mathrm{H}), 7.20$ $-7.14(\mathrm{~m}, 2 \mathrm{H}), 6.51(\mathrm{~s}, 1 \mathrm{H}), 4.35(\mathrm{~s}, 2 \mathrm{H}), 3.80(\mathrm{~s}, 2 \mathrm{H}), 1.39(\mathrm{~s}, 9 \mathrm{H}) \mathrm{ppm}$.

${ }^{13} \mathrm{C}$ NMR $\left(151 \mathrm{MHz}, \mathrm{C}_{6} \mathrm{D}_{6}\right) \delta 13 \mathrm{C}$ NMR $(151 \mathrm{MHz}, \mathrm{C} 6 \mathrm{D} 6) \delta$ 167.4, 137.2, 125.7, 123.0, 121.8, $120.1,118.6,114.7,111.9,58.1,44.3,41.8,28.4 \mathrm{ppm}$.<smiles>Nc1ccnc([N+](=O)[O-])c1CO</smiles>

\section{(2,4-dimethylpyridin-3-yl)methanol (S5):}

Lithium aluminum hydride ( $4.0 \mathrm{M}$ in ether, $7.5 \mathrm{~mL}, 10.0 \mathrm{mmol}, 2.0$ equiv) was added to cold, dry THF (12 mL). Ethyl 2,4-dimethylnicotinate ( $896.1 \mathrm{mg}, 5.0 \mathrm{mmol}, 1.0$ equiv) was dissolved in dry THF $(3 \mathrm{~mL})$ and slowly added to the lithium aluminum hydride solution at $0^{\circ} \mathrm{C}$. The reaction mixture was warmed to room temperature and stirred for 2 hours, then cooled to $0^{\circ} \mathrm{C}$, diluted with water, quenched with $1 \mathrm{M} \mathrm{NaOH}(\mathrm{aq})$, filtered through a plug of celite, and extracted with EtOAc (3x). The combined organic layers were dried over $\mathrm{Na}_{2} \mathrm{SO}_{4}$, filtered, and concentrated under reduced pressure to afford the title compound as a tan amorphous solid (519 $\mathrm{mg}, 76 \%)$.

${ }^{1} \mathbf{H}$ NMR $(600 \mathrm{MHz}, \mathrm{CDCl}) \delta 8.26(\mathrm{~d}, \mathrm{~J}=5.0 \mathrm{~Hz}, 1 \mathrm{H}), 6.96(\mathrm{~d}, \mathrm{~J}=5.1 \mathrm{~Hz}, 1 \mathrm{H}), 4.77(\mathrm{~s}, 2 \mathrm{H})$, 2.63 (s, 3H), 2.42 (s, 3H) ppm.

${ }^{13}$ C NMR (151 MHz, CDCl3) $\delta 157.7,148.4,147.0,132.2,124.0,59.0,22.4,19.1 \mathrm{ppm}$.

LRMS (EI) m/z: [M] ${ }^{+}$calc'd. for $\mathrm{C}_{8} \mathrm{H}_{11} \mathrm{NO}, 137.1$, found 137.0.<smiles>Cc1ccnc(C)c1C=O</smiles>

\section{2,4-dimethylnicotinaldehyde (S6):}

(2,4-dimethylpyridin-3-yl)methanol (S5) (478.8 $\mathrm{mg}, 3.5 \mathrm{mmol}, 1.0$ equiv), and manganese dioxide (1520 mg, $20.0 \mathrm{mmol}, 5.0$ equiv) were dissolved in $\mathrm{CH}_{2} \mathrm{Cl}_{2}(32 \mathrm{~mL}, 0.15 \mathrm{M})$ and refluxed at $40^{\circ} \mathrm{C}$ 
for 18 hours. The reaction mixture was cooled to room temperature and filtered through a plug of celite. Purification on silica gel (20-50\% EtOAc/hexanes) afforded the title compound as a brown amorphous solid $(263 \mathrm{mg}, 56 \%)$.

${ }^{1} \mathbf{H}$ NMR $(600 \mathrm{MHz}, \mathrm{CDCl} 3) \delta 10.64(\mathrm{~s}, 1 \mathrm{H}), 8.47(\mathrm{~d}, \mathrm{~J}=5.1 \mathrm{~Hz}, 1 \mathrm{H}), 7.06(\mathrm{~d}, \mathrm{~J}=5.2 \mathrm{~Hz}, 1 \mathrm{H})$, $2.83(\mathrm{~s}, 3 \mathrm{H}), 2.61(\mathrm{~s}, 3 \mathrm{H}) \mathrm{ppm}$.

${ }^{13}$ C NMR (151 MHz, CDCl3) $\delta$ 192.7, 160.9, 152.2, 150.1, 128.6, 125.3, 23.3, 20.4 ppm.

LRMS (EI) m/z: [M] ${ }^{+}$calc'd. for $\mathrm{C}_{8} \mathrm{H}_{9} \mathrm{NO}, 135.1$, found 135.0.<smiles>Cc1ccnc(C)c1CNC(C)(C)C</smiles>

$N$-((2,4-dimethylpyridin-3-yl)methyl)-2-methylpropan-2-amine (S7):

Prepared according to General Reductive Amination Procedure. 2,4-dimethylnicotinaldehyde (S6) (237 mg, $1.8 \mathrm{mmol}, 1.0$ equiv) and tert-butyl amine $(0.28 \mathrm{~mL}, 2.6 \mathrm{mmol}, 1.5$ equiv) were stirred in $\mathrm{MeOH}(9 \mathrm{~mL}, 0.2 \mathrm{M})$ for 16 hours. After $\mathrm{NaBH}_{4}(100 \mathrm{mg}, 2.6 \mathrm{mmol}, 1.5$ equiv) was added, the reaction was stirred for an additional 1 hour. The title compound was obtained as a white amorphous solid (316 mg, 94\%).

${ }^{1} \mathbf{H}$ NMR $(600 \mathrm{MHz}, \mathrm{CDCl} 3) \delta 8.22(\mathrm{~d}, \mathrm{~J}=5.0 \mathrm{~Hz}, 1 \mathrm{H}), 6.92(\mathrm{~d}, \mathrm{~J}=5.0 \mathrm{~Hz}, 1 \mathrm{H}), 3.72(\mathrm{~s}, 2 \mathrm{H})$, $2.61(\mathrm{~s}, 3 \mathrm{H}), 2.38$ (s, 3H), $1.20(\mathrm{~s}, 9 \mathrm{H}) \mathrm{ppm}$.

${ }^{13}$ C NMR (151 MHz, CDCl3) $\delta 157.2,147.3,146.4,132.7,123.7,50.7,40.4,28.9,22.1,18.9$ ppm.

LRMS (EI) m/z: [M] $]^{+}$calc'd. for $\mathrm{C}_{12} \mathrm{H}_{20} \mathrm{~N}_{2}, 192.2$, found 192.2.<smiles>Cc1ccnc(C)c1CN(C(=O)CCl)C(C)(C)C</smiles>

$\boldsymbol{N}$-(tert-butyl)-2-chloro- $\boldsymbol{N}$-((2,4-dimethylpyridin-3-yl)methyl)acetamide (S8):

Prepared according to General Acylation Procedure. N-((2,4-dimethylpyridin-3-yl)methyl)-2methylpropan-2-amine (S7) (289 mg, $1.5 \mathrm{mmol}, 1.0$ equiv), Et ${ }_{3} \mathrm{~N}$ (0.23 mL, $1.7 \mathrm{mmol}, 1.1$ equiv), and chloroacetyl chloride $(0.13 \mathrm{~mL}, 1.7 \mathrm{mmol}, 1.1$ equiv) were stirred in THF (15 mL, $0.1 \mathrm{M})$ for 3 hours. The title compound was afforded as a white amorphous solid (148 mg, 37\%).

${ }^{1} \mathbf{H}$ NMR $(600 \mathrm{MHz}, \mathrm{CDCl} 3) \delta 8.28(\mathrm{~d}, \mathrm{~J}=5.0 \mathrm{~Hz}, 1 \mathrm{H}), 6.94(\mathrm{~d}, \mathrm{~J}=4.9 \mathrm{~Hz}, 1 \mathrm{H}), 4.69$ (s, 2H), $4.15(\mathrm{~s}, 2 \mathrm{H}), 2.59(\mathrm{~s}, 3 \mathrm{H}), 2.38(\mathrm{~s}, 3 \mathrm{H}), 1.38(\mathrm{~s}, 9 \mathrm{H}) \mathrm{ppm}$.

${ }^{13}$ C NMR (151 MHz, CDCl3) $\delta$ 168.6, 155.9, 147.6, 145.4, 130.9, 125.2, 59.4, 46.0, 44.9, 28.4, 23.9, $20.5 \mathrm{ppm}$.

LRMS (EI) m/z: [M] calc'd. for $\mathrm{C}_{14} \mathrm{H}_{21} \mathrm{ClN}_{2} \mathrm{O}$, 268.1, found 268.1.<smiles>CC(C)(C)NCc1cccc(C#N)c1</smiles>

3-((tert-butylamino)methyl)benzonitrile (S9):

Prepared according to General Reductive Amination Procedure. 3-formylbenzonitrile (525 mg, 4.0 mmol, 1.0 equiv) and tert-butyl amine ( $0.73 \mathrm{~mL}, 4.8 \mathrm{mmol}, 1.2$ equiv) were stirred in $\mathrm{MeOH}(20$ 
$\mathrm{mL}, 0.2 \mathrm{M}$ ) for 12 hours. After $\mathrm{NaBH}_{4}(227 \mathrm{mg}, 6.0 \mathrm{mmol}, 1.5$ equiv) was added, the reaction was stirred for an additional 2 hours. The title compound was obtained as a colorless oil (694 mg, 92\%). ${ }^{1} \mathbf{H}$ NMR $(600 \mathrm{MHz}, \mathrm{CDCl} 3) \delta 7.65(\mathrm{~s}, 1 \mathrm{H}), 7.56(\mathrm{~d}, \mathrm{~J}=8.3 \mathrm{~Hz}, 1 \mathrm{H}), 7.47(\mathrm{~d}, \mathrm{~J}=7.7 \mathrm{~Hz}, 1 \mathrm{H})$, $7.37(\mathrm{t}, \mathrm{J}=7.7 \mathrm{~Hz}, 1 \mathrm{H}), 3.73(\mathrm{~s}, 2 \mathrm{H}), 1.14(\mathrm{~s}, 9 \mathrm{H}) \mathrm{ppm}$.

${ }^{13}$ C NMR (151 MHz, CDCl3) $\delta$ 143.4, 132.7, 131.8, 130.5, 129.1, 119.1, 112.3, 50.9, 46.5, 29.2 ppm.

LRMS (EI) m/z: [M] $]^{+}$calc'd. for $\mathrm{C}_{12} \mathrm{H}_{16} \mathrm{~N}_{2}, 188.1$, found 188.2.<smiles>CC(C)(C)N(Cc1cccc(C#N)c1)C(=O)CCl</smiles>

$N$-(tert-butyl)-2-chloro- $N$-(3-cyanobenzyl)acetamide (S10):

Prepared according to General Acylation Procedure. 3-((tert-butylamino)methyl)benzonitrile (S9) (377 mg, $2.0 \mathrm{mmol}, 1.0$ equiv), $\mathrm{Et}_{3} \mathrm{~N}$ (0.31 mL, $2.2 \mathrm{mmol}, 1.1$ equiv), and chloroacetyl chloride $\left(0.18 \mathrm{~mL}, 2.2 \mathrm{mmol}, 1.1\right.$ equiv) were stirred in $\mathrm{CH}_{2} \mathrm{Cl}_{2}(20 \mathrm{~mL}, 0.1 \mathrm{M})$ for 3 hours. The title compound was afforded as a tan soid (526 mg, 99\%).

${ }^{1} \mathbf{H}$ NMR $(600 \mathrm{MHz}, \mathrm{CDCl} 3) \delta 7.59(\mathrm{~d}, \mathrm{~J}=7.6 \mathrm{~Hz}, 1 \mathrm{H}), 7.54-7.49(\mathrm{~m}, 2 \mathrm{H}), 7.47$ (d, J = $7.7 \mathrm{~Hz}$, $1 \mathrm{H}), 4.69$ (s, 2H), $3.92(\mathrm{~s}, 2 \mathrm{H}), 1.43(\mathrm{~s}, 9 \mathrm{H}) \mathrm{ppm}$.

${ }^{13}$ C NMR $(151 \mathrm{MHz}, \mathrm{CDCl} 3) \delta 167.7,140.7,131.4,130.1,130.0,129.2,118.5,113.5,59.1,48.4$, 44.0, $28.6 \mathrm{ppm}$.

LRMS (EI) m/z: [M] ${ }^{+}$calc'd. for $\mathrm{C}_{14} \mathrm{H}_{17} \mathrm{ClN}_{2} \mathrm{O}$, 264.1, found 264.1.<smiles>CC(C)(C)NCc1cccc(C(F)(F)F)c1</smiles>

\section{2-methyl- $\boldsymbol{N}$-(3-(trifluoromethyl)benzyl)propan-2-amine (S11):}

Prepared according to General Reductive Amination Procedure. 3-(trifluoromethyl)benzaldehyde ( $0.54 \mathrm{~mL}, 4.0 \mathrm{mmol}, 1.0$ equiv) and tert-butyl amine $(0.50 \mathrm{~mL}, 4.8 \mathrm{mmol}, 1.2$ equiv) were stirred in $\mathrm{MeOH}\left(20 \mathrm{~mL}, 0.2 \mathrm{M}\right.$ ) for 26 hours. After $\mathrm{NaBH}_{4}(227 \mathrm{mg}, 6.0 \mathrm{mmol}, 1.5$ equiv) was added, the reaction was stirred for an additional $30 \mathrm{~min}$. The title compound was obtained as a lightyellow oil (590 mg, 86\%).

${ }^{1} \mathbf{H}$ NMR $\left(400 \mathrm{MHz}, \mathrm{CDCl}_{3}\right) \delta 7.62(\mathrm{~s}, 1 \mathrm{H}), 7.54(\mathrm{~d}, \mathrm{~J}=7.3 \mathrm{~Hz}, 1 \mathrm{H}), 7.49(\mathrm{~d}, \mathrm{~J}=7.5 \mathrm{~Hz}, 1 \mathrm{H}), 7.44$ $-7.39(\mathrm{~m}, 1 \mathrm{H}), 3.79(\mathrm{~s}, 2 \mathrm{H}), 1.18(\mathrm{~s}, 9 \mathrm{H}) \mathrm{ppm}$.

${ }^{13} \mathbf{C}$ NMR (151 MHz, $\left.\mathrm{CDCl}_{3}\right) 142.5,131.7,130.7\left(\mathrm{q},{ }^{2} \mathrm{~J}_{\mathrm{C}-\mathrm{F}}=32.1 \mathrm{~Hz}\right), 128.8,125.0\left(\mathrm{q},{ }^{3} \mathrm{~J}_{\mathrm{C}-\mathrm{F}}=3.9\right.$ $\mathrm{Hz}), 124.3\left(\mathrm{q},{ }^{1} \mathrm{~J}_{\mathrm{C}-\mathrm{F}}=272.2 \mathrm{~Hz}\right), 123.7\left(\mathrm{q},{ }^{3} \mathrm{~J}_{\mathrm{C}-\mathrm{F}}=3.9 \mathrm{~Hz}\right), 50.9,46.8,29.2 \mathrm{ppm}$.

LRMS (EI) m/z: [M] calc'd. for $\mathrm{C}_{12} \mathrm{H}_{16} \mathrm{~F}_{3} \mathrm{~N}$, 231.1, found 231.2.<smiles>CC(C)(C)N(Cc1cccc(C(F)(F)F)c1)C(=O)CCl</smiles>

$N$-(tert-butyl)-2-chloro- $N$-(3-(trifluoromethyl)benzyl)acetamide (S12):

Prepared according to General Acylation Procedure. 2-methyl- $\mathrm{N}$-(3(trifluoromethyl)benzyl)propan-2-amine (S11) (490 mg, $3.0 \mathrm{mmol}, 1.0$ equiv), $\mathrm{Et}_{3} \mathrm{~N}$ (0.50 mL, 3.6 mmol, 1.2 equiv), and chloroacetyl chloride $\left(0.29 \mathrm{~mL}, 3.6 \mathrm{mmol}, 1.2\right.$ equiv) were stirred in $\mathrm{CH}_{2} \mathrm{Cl}_{2}$ 
(30 mL, $0.1 \mathrm{M}$ ) for 1 hour. The title compound afforded as a light-yellow amorphous solid (685 $\mathrm{mg}, 95 \%)$.

${ }^{1} \mathbf{H}$ NMR $\left(600 \mathrm{MHz}, \mathrm{CDCl}_{3}\right) \delta 7.57(\mathrm{~d}, \mathrm{~J}=7.7 \mathrm{~Hz}, 1 \mathrm{H}), 7.52(\mathrm{t}, \mathrm{J}=7.7 \mathrm{~Hz}, 1 \mathrm{H}), 7.47(\mathrm{~s}, 1 \mathrm{H}), 7.42$ $(\mathrm{d}, \mathrm{J}=7.6 \mathrm{~Hz}, 1 \mathrm{H}), 4.73(\mathrm{~s}, 2 \mathrm{H}), 3.94(\mathrm{~s}, 2 \mathrm{H}), 1.46(\mathrm{~s}, 9 \mathrm{H}) \mathrm{ppm}$.

${ }^{1} \mathrm{H}$ NMR spectrum is consistent with reported values. ${ }^{9}$<smiles>CC(C)(C)NCc1cc(C(F)(F)F)cc(C(F)(F)F)c1</smiles>

\section{$N$-(3,5-bis(trifluoromethyl)benzyl)-2-methylpropan-2-amine (S13):}

Prepared according to General Reductive Amination Procedure. 3,5bis(trifluoromethyl)benzaldehyde $(0.66 \mathrm{~mL}, 4.0 \mathrm{mmol}, 1.0$ equiv) and tert-butyl amine $(0.50 \mathrm{~mL}$, $4.8 \mathrm{mmol}, 1.2$ equiv) were stirred in $\mathrm{MeOH}(20 \mathrm{~mL}, 0.2 \mathrm{M})$ for 17 hours. After $\mathrm{NaBH}_{4}(227 \mathrm{mg}$, $6.0 \mathrm{mmol}, 1.5$ equiv) was added, the reaction was stirred for an additional $30 \mathrm{~min}$. The title compound was obtained as a colorless oil (164 $\mathrm{mg}, 14 \%)$.

${ }^{1} \mathbf{H}$ NMR (400 MHz, CDCl 3 ) $\delta 7.84(\mathrm{~s}, 2 \mathrm{H}), 7.74(\mathrm{~s}, 1 \mathrm{H}), 3.86(\mathrm{~s}, 2 \mathrm{H}), 1.18(\mathrm{~s}, 9 \mathrm{H}) \mathrm{ppm}$.

${ }^{13} \mathbf{C ~ N M R}\left(151 \mathrm{MHz}, \mathrm{CDCl}_{3}\right) \delta 131.6\left(\mathrm{q},{ }^{2} \mathrm{~J}_{\mathrm{C}-\mathrm{F}}=33.1 \mathrm{~Hz}\right), 128.6,123.6\left(\mathrm{q},{ }^{1} \mathrm{~J}_{\mathrm{C}-\mathrm{F}}=272.5 \mathrm{~Hz}\right), 121.0$, 51.4, 46.5, $29.2 \mathrm{ppm}$.

LRMS (EI) m/z: [M] $]^{+}$calc'd. for $\mathrm{C}_{13} \mathrm{H}_{15} \mathrm{~F}_{6} \mathrm{~N}, 299.1$, found 299.1 .<smiles>CN(Cc1cc(C(F)(F)F)cc(C(F)(F)F)c1)C(=O)CCl</smiles>

$N$-(3,5-bis(trifluoromethyl)benzyl)- $N$-(tert-butyl)-2-chloroacetamide (S14):

Prepared according to General Acylation Procedure. $N$-(3,5-bis(trifluoromethyl)benzyl)-2methylpropan-2-amine (S13) (157 mg, $0.52 \mathrm{mmol}, 1.0$ equiv), Et $3 \mathrm{~N}$ ( $84 \mu \mathrm{L}, 0.62 \mathrm{mmol}, 1.2$ equiv), and chloroacetyl chloride ( $49 \mu \mathrm{L}, 0.62 \mathrm{mmol}, 1.2$ equiv) were stirred in $\mathrm{CH}_{2} \mathrm{Cl}_{2}(5 \mathrm{~mL}, 0.1 \mathrm{M})$ for 1 hour. The title compound afforded as a light-yellow amorphous solid (180 mg, 92\%).

${ }^{1} \mathbf{H}$ NMR $\left(600 \mathrm{MHz}, \mathrm{CDCl}_{3}\right) \delta 7.83(\mathrm{~s}, 1 \mathrm{H}), 7.69(\mathrm{~s}, 2 \mathrm{H}), 4.80(\mathrm{~s}, 2 \mathrm{H}), 3.92(\mathrm{~s}, 2 \mathrm{H}), 1.45(\mathrm{~s}, 9 \mathrm{H})$ ppm.

${ }^{13} \mathbf{C}$ NMR $\left(151 \mathrm{MHz}, \mathrm{CDCl}_{3}\right) \delta 167.8,142.0,132.8\left(\mathrm{q},{ }^{2} \mathrm{~J}_{\mathrm{C}-\mathrm{F}}=33.5 \mathrm{~Hz}\right), 125.9\left(\mathrm{q},{ }^{3} \mathrm{~J}_{\mathrm{C}-\mathrm{F}}=3.8 \mathrm{~Hz}\right)$, $123.2\left(\mathrm{q},{ }^{1} \mathrm{~J}_{\mathrm{C}-\mathrm{F}}=272.9 \mathrm{~Hz}\right), 121.8\left(\right.$ hept, $\left.{ }^{3} \mathrm{~J}_{\mathrm{C}-\mathrm{F}}=3.8 \mathrm{~Hz}\right), 59.2,48.6,43.8,28.6 \mathrm{ppm}$.

LRMS (EI) m/z: [M] ${ }^{+}$calc'd. for $\mathrm{C}_{15} \mathrm{H}_{16} \mathrm{ClF}_{6} \mathrm{NO}, 375.1$, found 375.1.

\footnotetext{
${ }^{9}$ Yanagita, H.; Urano, E.; Matsumoto, K.; Ichikawa, R.; Takaesu, Y.; Ogata, M.; Murakami, T.; Wu, H.; Chiba, J.; Komano, J.; Hoshino, T. Structural and biochemical study on the inhibitory activity of derivatives of 5-nitro-furan2-carboxylic acid for RNase H function of HIV-1 reverse transcriptase. Bioorg. Med. Chem. 2011, 19, 816-825.
} 
<smiles>COc1cc(CNC(C)(C)C)cc(OC)c1</smiles>

$N$-(3,5-dimethoxybenzyl)-2-methylpropan-2-amine (S15):

Prepared according to General Reductive Amination Procedure. 3,5-dimethoxybenzaldehyde (665 $\mathrm{mg}, 4.0 \mathrm{mmol}, 1.0$ equiv) and tert-butyl amine $(0.50 \mathrm{~mL}, 4.8 \mathrm{mmol}, 1.2$ equiv) were stirred in $\mathrm{MeOH}\left(20 \mathrm{~mL}, 0.2 \mathrm{M}\right.$ ) for 2 hours. After $\mathrm{NaBH}_{4}(227 \mathrm{mg}, 6.0 \mathrm{mmol}, 1.5$ equiv) was added, the reaction was stirred for an additional 1 hour. The title compound was obtained as a white amorphous solid (701 mg, 78\%).

${ }^{1} \mathbf{H}$ NMR $\left(400 \mathrm{MHz}, \mathrm{CDCl}_{3}\right) \delta 6.51(\mathrm{~d}, \mathrm{~J}=2.1 \mathrm{~Hz}, 2 \mathrm{H}), 6.34(\mathrm{t}, \mathrm{J}=2.3 \mathrm{~Hz}, 1 \mathrm{H}), 3.79(\mathrm{~s}, 6 \mathrm{H}), 3.67$ (s, 2H), 1.17 (s, 9H) ppm.

${ }^{1} \mathrm{H}$ NMR is consistent with reported values. ${ }^{10}$<smiles>COc1cc(CN(C(=O)CCl)C(C)(C)C)cc(OC)c1</smiles>

$N$-(tert-butyl)-2-chloro- $N$-(3,5-dimethoxybenzyl)acetamide (S16):

Prepared according to General Acylation Procedure. N-(3,5-dimethoxybenzyl)-2-methylpropan-2amine (S15) (670 mg, $3.0 \mathrm{mmol}, 1.0$ equiv), $\mathrm{Et}_{3} \mathrm{~N}$ (0.50 mL, $3.6 \mathrm{mmol}, 1.2$ equiv), and chloroacetyl chloride $\left(0.29 \mathrm{~mL}, 3.6 \mathrm{mmol}, 1.2\right.$ equiv) were stirred in $\mathrm{CH}_{2} \mathrm{Cl}_{2}(30 \mathrm{~mL}, 0.1 \mathrm{M})$ for 1 hour. The title compound was obtained as a light-brown amorphous solid (899 $\mathrm{mg}, 100 \%)$.

${ }^{1} \mathbf{H}$ NMR $\left(400 \mathrm{MHz}, \mathrm{CDCl}_{3}\right) \delta 6.36(\mathrm{t}, \mathrm{J}=2.2 \mathrm{~Hz}, 1 \mathrm{H}), 6.34(\mathrm{~d}, \mathrm{~J}=2.2 \mathrm{~Hz}, 2 \mathrm{H}), 4.59(\mathrm{~d}, \mathrm{~J}=0.8$ $\mathrm{Hz}, 2 \mathrm{H}), 3.97$ (s, 2H), 3.78 (s, 6H), 1.47 (s, 9H) ppm.

${ }^{1} \mathrm{H}$ NMR spectrum is consistent with reported values. ${ }^{11}$<smiles>Cc1ccccc1CNC(C)(C)C</smiles>

2-methyl- $N$-(2-methylbenzyl)propan-2-amine (S17):

Prepared according to General Reductive Amination Procedure. 2-methylbenzaldehyde $(0.46 \mathrm{~mL}$, $4.0 \mathrm{mmol}, 1.0$ equiv) and tert-butyl amine $(0.73 \mathrm{~mL}, 4.8 \mathrm{mmol}, 1.2$ equiv) were stirred in $\mathrm{MeOH}$ $(20 \mathrm{~mL}, 0.2 \mathrm{M})$ for 3 hours. After $\mathrm{NaBH}_{4}(227 \mathrm{mg}, 6.0 \mathrm{mmol}, 1.5$ equiv) was added, the reaction was stirred for an additional 2 hours. The title compound was obtained as a white amorphous solid (546 mg, 77\%).

${ }^{1} \mathbf{H}$ NMR (400 MHz, cdcl3) $\delta 7.33-7.24(\mathrm{~m}, 1 \mathrm{H}), 7.21-7.11(\mathrm{~m}, 3 \mathrm{H}), 3.70(\mathrm{~s}, 2 \mathrm{H}), 2.37$ (s, 3H), $1.20(\mathrm{~s}, 9 \mathrm{H}) \mathrm{ppm}$.

\footnotetext{
${ }^{10}$ Padwa, A.; Kuethe, J. T. Additive and Vinylogous Pummerer Reactions of Amido Sulfoxides and Their Use in the Preparation of Nitrogen Containing Heterocycles. J. Org. Chem. 1998, 63, 4256-4268.

${ }^{11}$ Hamada, T.; Okuno, Y.; Ohmori, M.; Nishi, T.; Yonemitsu, O. Photochemical Synthesis of 1,2,3,4-

Tetrahydroisoquinolin-3-ones and Oxindoles from N-Chloroacetyl Derivatives of Benzylamines and Anilines. Role of Intramolecular Exciplex Formation and cis Conformation of Amide Bonds. Chem. Pharm. Bull. 1981, 29, 128136.
} 
${ }^{1} \mathrm{H}$ NMR spectrum is consistent with reported values. ${ }^{12}$<smiles>CC(C)(C)N(Cc1ccccc1[N+](=O)[O-])C(=O)CCl</smiles>

$\boldsymbol{N}$-(tert-butyl)-2-chloro- $\boldsymbol{N}$-(2-methylbenzyl)acetamide (S18):

Prepared according to General Acylation Procedure. 2-methyl- $N$-(2-methylbenzyl)propan-2amine (S17) (319 mg, $1.8 \mathrm{mmol}, 1.0$ equiv), $\mathrm{Et}_{3} \mathrm{~N}$ (0.28 mL, $2.0 \mathrm{mmol}, 1.1$ equiv), and chloroacetyl chloride $\left(0.16 \mathrm{~mL}, 2.0 \mathrm{mmol}, 1.1\right.$ equiv) were stirred in $\mathrm{CH}_{2} \mathrm{Cl}_{2}(18 \mathrm{~mL}, 0.1 \mathrm{M})$ for 3.5 hours, which afforded the title compound as a white amorphous solid (401 $\mathrm{mg}, 88 \%)$.

${ }^{1} \mathbf{H}$ NMR (600 MHz, CDCl3) $\delta 7.25$ - 7.15 (m, 4H), 4.54 (s, 2H), 3.89 (s, 2H), 2.29 (s, 3H), 1.46 (s, 9H) ppm.

${ }^{13}$ C NMR (151 MHz, CDCl3) $\delta 167.8,136.3,134.3,130.7,127.3,127.3,126.7,124.6,58.6,46.7$, 44.1, 28.3, $19.1 \mathrm{ppm}$.

LRMS (EI) m/z: [M] ${ }^{+}$calc'd. for $\mathrm{C}_{14} \mathrm{H}_{20} \mathrm{ClNO}$, 253.1, found 253.2.<smiles>Cc1cccc(C)c1CNC(C)(C)C</smiles>

$N$-(2,6-dimethylbenzyl)-2-methylpropan-2-amine (S19):

Prepared according to General Reductive Amination Procedure. 2,6-dimethylbenzaldehyde (537 $\mathrm{mg}, 4.0 \mathrm{mmol}, 1.0$ equiv) and tert-butyl amine $(1.20 \mathrm{~mL}, 8.0 \mathrm{mmol}, 2.0$ equiv) were stirred in $\mathrm{MeOH}\left(20 \mathrm{~mL}, 0.2 \mathrm{M}\right.$ ) for 6 hours. After $\mathrm{NaBH}_{4}(227 \mathrm{mg}, 6.0 \mathrm{mmol}, 1.5$ equiv) was added, the reaction was stirred for an additional 1.5 hours. The title compound was obtained as a white amorphous solid (708 $\mathrm{mg}, 93 \%)$.

${ }^{1} \mathbf{H}$ NMR (600 MHz, CDCl3) $\delta 7.07-6.98(\mathrm{~m}, 3 \mathrm{H}), 3.72(\mathrm{~s}, 2 \mathrm{H}), 2.39$ (s, 6H), 1.45 (s, 1H), 1.21 $(\mathrm{s}, 9 \mathrm{H})$.

${ }^{1} \mathrm{H}$ NMR spectrum is consistent with reported values. ${ }^{12}$<smiles>Cc1cccc(C)c1CN(C(=O)CCl)C(C)(C)C</smiles>

$\boldsymbol{N}$-(tert-butyl)-2-chloro- $\boldsymbol{N}$-(2,6-dimethylbenzyl)acetamide (S20):

Prepared according to General Acylation Procedure. $N$-(2,6-dimethylbenzyl)-2-methylpropan-2amine (S19) (574 mg, $3.0 \mathrm{mmol}, 1.0$ equiv), $\mathrm{Et}_{3} \mathrm{~N}$ ( $0.46 \mathrm{~mL}, 3.3 \mathrm{mmol}, 1.1$ equiv), and chloroacetyl chloride ( $0.26 \mathrm{~mL}, 3.3 \mathrm{mmol}, 1.1$ equiv) were stirred in $\mathrm{CH}_{2} \mathrm{Cl}_{2}(30 \mathrm{~mL}, 0.1 \mathrm{M})$ for 14 hours, which afforded the title compound as a yellow amorphous solid (654 $\mathrm{mg}, 81 \%)$.

${ }^{1} \mathbf{H}$ NMR (600 MHz, CDCl3) $\delta 7.08$ (t, J = 7.5 Hz, 1H), $7.00(\mathrm{~d}, \mathrm{~J}=7.5 \mathrm{~Hz}, 2 \mathrm{H}), 4.65$ (s, 2H), 4.11 $(\mathrm{s}, 2 \mathrm{H}), 2.36(\mathrm{~s}, 6 \mathrm{H}), 1.39(\mathrm{~s}, 9 \mathrm{H}) \mathrm{ppm}$.

\footnotetext{
${ }^{12}$ Franchi, P.; Casati, C.; Mezzina, E.; Lucarini, M. Kinetic control of the direction of inclusion of nitroxide cyclodextrines. Org. Biomol. Chem., 2011, 9, 6396-6401.
} 
${ }^{13}$ C NMR $(151 \mathrm{MHz}, \mathrm{CDCl} 3) \delta 168.6,136.0,134.8,130.1,127.4,59.5,46.8,45.2,28.3,28.3$, $21.1 \mathrm{ppm}$.

LRMS (EI) m/z: [M] ${ }^{+}$calc'd. for $\mathrm{C}_{15} \mathrm{H}_{22} \mathrm{ClNO}$, 267.1, found 267.1.<smiles>Cc1cccc(CNC(C)(C)C)c1</smiles>

\section{2-methyl- $N$-(3-methylbenzyl)propan-2-amine (S21):}

Prepared according to General Reductive Amination Procedure. 3-methylbenzaldehyde $(0.71 \mathrm{~mL}$, $6.0 \mathrm{mmol}, 1.0$ equiv) and tert-butyl amine $(1.10 \mathrm{~mL}, 7.2 \mathrm{mmol}, 1.2$ equiv) were stirred in $\mathrm{MeOH}$ $(30 \mathrm{~mL}, 0.2 \mathrm{M})$ for 15 hours. After $\mathrm{NaBH}_{4}(340 \mathrm{mg}, 9.0 \mathrm{mmol}, 1.5$ equiv) was added, the reaction was stirred for an additional 2 hours. The title compound was obtained as a light-yellow oil (990 $\mathrm{mg}, 93 \%)$.

${ }^{1} \mathbf{H}$ NMR $(400 \mathrm{MHz}, \mathrm{CDCl} 3) \delta 7.23-7.10(\mathrm{~m}, 3 \mathrm{H}), 7.04(\mathrm{~d}, \mathrm{~J}=7.5 \mathrm{~Hz}, 1 \mathrm{H}), 3.69(\mathrm{~s}, 2 \mathrm{H}), 2.34$ $(\mathrm{d}, \mathrm{J}=0.7 \mathrm{~Hz}, 3 \mathrm{H}), 1.18$ (s, 9H) ppm.

${ }^{1} \mathrm{H}$ NMR spectrum is consistent with reported values. ${ }^{13}$<smiles>Cc1cccc(CN(C)C(=O)CCl)c1</smiles>

$N$-(tert-butyl)-2-chloro- $N$-(3-methylbenzyl)acetamide (S22):

Prepared according to General Reductive Amination Procedure. 2-methyl- $\mathrm{N}$-(3methylbenzyl)propan-2-amine (S21) (443 mg, $2.5 \mathrm{mmol}, 1.0$ equiv) $\mathrm{Et}_{3} \mathrm{~N}(0.38 \mathrm{~mL}, 2.8 \mathrm{mmol}, 1.1$ equiv), and chloroacetyl chloride ( $0.22 \mathrm{~mL}, 2.8 \mathrm{mmol}, 1.1$ equiv) were stirred in $\mathrm{CH}_{2} \mathrm{Cl}_{2}(25 \mathrm{~mL}$, $0.1 \mathrm{M})$ for 1 hour, which afforded the title compound as a white amorphous solid (593 $\mathrm{mg}, 94 \%)$. ${ }^{1} \mathbf{H}$ NMR $(600 \mathrm{MHz}, \mathrm{CDCl} 3) \delta 7.21(\mathrm{t}, \mathrm{J}=7.5 \mathrm{~Hz}, 1 \mathrm{H}), 7.04(\mathrm{~d}, \mathrm{~J}=7.6 \mathrm{~Hz}, 1 \mathrm{H}), 6.97(\mathrm{~s}, 1 \mathrm{H}), 6.96$ (d, J = 8.5 Hz, 1H), 4.59 (s, 2H), $3.95(\mathrm{~s}, 2 \mathrm{H}), 2.31(\mathrm{~s}, 3 \mathrm{H}), 1.42$ (s, 9H) ppm.

${ }^{13}$ C NMR (151 MHz, CDCl3) $\delta 167.5,138.7,138.4,128.9,128.1,126.0,122.3,58.6,48.6,44.2$, 28.3, $21.4 \mathrm{ppm}$.

LRMS (EI) m/z: [M] calc'd. for $\mathrm{C}_{14} \mathrm{H}_{20} \mathrm{ClNO}$, 253.1, found 253.2.<smiles>CC(C)(C)NCc1ccccc1</smiles>

\section{$N$-benzyl-2-methylpropan-2-amine (S23):}

Prepared according to General Reductive Amination Procedure. Benzaldehyde (1.02 mL, 10.0 mmol, 1.0 equiv) and tert-butyl amine $(1.26 \mathrm{~mL}, 12.0 \mathrm{mmol}, 1.2$ equiv) were stirred in $\mathrm{MeOH}$ (50 $\mathrm{mL}, 0.2 \mathrm{M})$ for 4 hours. After $\mathrm{NaBH}_{4}(567 \mathrm{mg}, 15.0 \mathrm{mmol}, 1.5$ equiv) was added, the reaction was stirred for an additional 1 hour. The title compound was obtained as a colorless oil (1061 mg, $100 \%)$.

\footnotetext{
${ }^{13}$ Jankowski, K.; Harvey, R. A general one-pot, three-component mono $\mathrm{N}$-alkylation of amines and amine derivatives in lithium perchlorate/diethyl ether solution. Synthesis, 2005, 4, 627-633.
} 
${ }^{1}$ H NMR (400 MHz, cdcl3) $\delta 7.38-7.28(\mathrm{~m}, 4 \mathrm{H}), 7.25-7.20(\mathrm{~m}, 1 \mathrm{H}), 3.73(\mathrm{~s}, 2 \mathrm{H}), 1.18(\mathrm{~s}, 9 \mathrm{H})$ ppm.

${ }^{1} \mathrm{H}$ NMR spectrum is consistent with reported values. ${ }^{14}$<smiles>[14CH3]N(Cc1ccccc1)C(=O)[CH]Cl</smiles>

\section{$N$-benzyl- $N$-(tert-butyl)-2-chloropropanamide (S24):}

Prepared according to General Acylation Procedure. $N$-benzyl-2-methylpropan-2-amine (S23) (264 mg, $1.6 \mathrm{mmol}, 1.0$ equiv), $\mathrm{Et}_{3} \mathrm{~N}(0.26 \mathrm{~mL}, 1.9 \mathrm{mmol}, 1.2$ equiv), and 2-chloropropionyl chloride $\left(0.18 \mathrm{~mL}, 1.9 \mathrm{mmol}, 1.2\right.$ equiv) were stirred in $\mathrm{CH}_{2} \mathrm{Cl}_{2}(16 \mathrm{~mL}, 0.1 \mathrm{M})$ for 1 hour. Purification on silica gel (10\% EtOAc/hexanes) afforded the title compound as a white amorphous solid (276 mg, 68\%).

${ }^{1} \mathbf{H}$ NMR $\left(600 \mathrm{MHz}, \mathrm{CDCl}_{3}\right) \delta 7.38(\mathrm{t}, \mathrm{J}=7.7 \mathrm{~Hz}, 2 \mathrm{H}), 7.28(\mathrm{t}, \mathrm{J}=7.4 \mathrm{~Hz}, 1 \mathrm{H}), 7.17(\mathrm{~d}, \mathrm{~J}=7.2$ $\mathrm{Hz}, 2 \mathrm{H}), 4.82(\mathrm{~d}, \mathrm{~J}=18.9 \mathrm{~Hz}, 1 \mathrm{H}), 4.62(\mathrm{~d}, \mathrm{~J}=18.9 \mathrm{~Hz}, 1 \mathrm{H}), 4.32(\mathrm{q}, \mathrm{J}=6.4 \mathrm{~Hz}, 1 \mathrm{H}), 1.60(\mathrm{~d}, \mathrm{~J}=$ $6.4 \mathrm{~Hz}, 3 \mathrm{H}), 1.45$ (s, 9H) ppm.

${ }^{13}$ C NMR $\left(151 \mathrm{MHz}, \mathrm{CDCl}_{3}\right) \delta 170.5,139.2,129.1,127.3,125.3,58.5,52.1,48.3,28.4,21.4 \mathrm{ppm}$. LRMS (EI) m/z: [M] calc'd. for $\mathrm{C}_{14} \mathrm{H}_{20} \mathrm{ClNO}$, 253.1, found 253.2.<smiles>CC(NC(C)(C)C)c1ccccc1</smiles>

\section{2-methyl- $N$-(1-phenylethyl)propan-2-amine (S25):}

To a round bottomed flask charged with (1-bromoethyl)benzene $(0.41 \mathrm{~mL}, 3.0 \mathrm{mmol}, 1.0$ equiv) was added $\mathrm{MeCN}(15 \mathrm{~mL}, 0.2 \mathrm{M})$, tert-butylamine (0.38 mL, $3.6 \mathrm{mmol}, 1.2$ equiv), and $\mathrm{K}_{2} \mathrm{CO}_{3}$ ( $829 \mathrm{mg}, 6.0 \mathrm{mmol}, 2.0$ equiv). The resulting suspension was heated at reflux for 16 hours. After cooling to room temperature, the reaction was quenched with $\mathrm{H}_{2} \mathrm{O}$ and extracted with EtOAc (3x). The combined organic layers were washed with $1 \mathrm{M} \mathrm{HCl}(\mathrm{aq})$, and the resulting aqueous layer was brought to $\mathrm{pH} 14$ with $2 \mathrm{M} \mathrm{NaOH}$ (aq) extracted with $\mathrm{CH}_{2} \mathrm{Cl}_{2}$ (3x). The combined organic layers were dried over $\mathrm{MgSO}_{4}$, filtered, and concentrated under reduced pressure to afford the title compound as a colorless oil ( $363 \mathrm{mg}, 68 \%)$.

${ }^{1} \mathbf{H}$ NMR $\left(400 \mathrm{MHz}, \mathrm{CDCl}_{3}\right) \delta 7.41-7.33(\mathrm{~m}, 2 \mathrm{H}), 7.33-7.23(\mathrm{~m}, 2 \mathrm{H}), 7.23-7.14(\mathrm{~m}, 1 \mathrm{H}), 3.95$ $(\mathrm{q}, \mathrm{J}=6.7 \mathrm{~Hz}, 1 \mathrm{H}), 1.31(\mathrm{~d}, \mathrm{~J}=6.7 \mathrm{~Hz}, 3 \mathrm{H}), 1.02(\mathrm{~s}, 9 \mathrm{H}) \mathrm{ppm}$.

${ }^{1} \mathrm{H}$ NMR spectrum is consistent with reported values. ${ }^{15}$

\footnotetext{
${ }^{14}$ Niu, Z.; Zhang, W.; Lan, P. C.; Aguila, B.; Ma, S. Promoting frustrated lewis pairs for heterogeneous chemoselective hydrogenation via the tailored pore environment within metal-organic frameworks. Angew. Chem. Int. Ed. 2019, 58, 7420-7424.

${ }^{15}$ Cliffe, I. A.; Crossley, R.; Shepherd, R. G. Sterically Hindered Lithium Dialkylamides; A Novel Synthesis of Lithium Dialkylamides from $N$ - $t$-Alkyl- $N$-benzylideneamines and the Isolation of Highly Hindered $s$-Alkyl- $t$ alkylamines. Synthesis 1985, 12, 1138-1140.
} 
<smiles>CC(C)(C)N(C(=O)CCl)c1ccccc1</smiles>

\section{$N$-(tert-butyl)-2-chloro- $N$-(1-phenylethyl)acetamide (S26):}

Prepared according to General Acylation Procedure. 2-methyl- $N$-(1-phenylethyl)propan-2-amine (S25) (266 mg, $1.5 \mathrm{mmol}, 1.0$ equiv), $\mathrm{Et}_{3} \mathrm{~N}$ (0.25 mL, $1.8 \mathrm{mmol}, 1.2$ equiv), and chloroacetyl chloride (0.14 mL, $1.8 \mathrm{mmol}, 1.2$ equiv) were stirred in $\mathrm{CH}_{2} \mathrm{Cl}_{2}(15 \mathrm{~mL}, 0.1 \mathrm{M})$ for 4 hours. Purification on silica gel (30\% EtOAc/hexanes) afforded the title compound as a light-yellow oil (292 mg, 77\%).

${ }^{1} \mathbf{H}$ NMR $\left(500 \mathrm{MHz}, \mathrm{CDCl}_{3}\right) \delta 7.38(\mathrm{dd}, \mathrm{J}=8.3,7.2 \mathrm{~Hz}, 2 \mathrm{H}), 7.33-7.26(\mathrm{~m}, 3 \mathrm{H}), 5.13(\mathrm{q}, \mathrm{J}=7.0$ $\mathrm{Hz}, 1 \mathrm{H}), 3.76(\mathrm{~d}, \mathrm{~J}=12.7 \mathrm{~Hz}, 1 \mathrm{H}), 3.45(\mathrm{~d}, \mathrm{~J}=11.9 \mathrm{~Hz}, 1 \mathrm{H}), 1.79(\mathrm{~d}, \mathrm{~J}=7.1 \mathrm{~Hz}, 3 \mathrm{H}), 1.56(\mathrm{~s}, 9 \mathrm{H})$ ppm.

${ }^{13} \mathrm{C}$ NMR $\left(101 \mathrm{MHz}, \mathrm{CDCl}_{3}\right) \delta 169.0,143.1,129.3,127.3,125.6,60.0,53.0,44.7,29.4,21.0 \mathrm{ppm}$. LRMS (EI) m/z: [M] ${ }^{+}$calc'd. for $\mathrm{C}_{14} \mathrm{H}_{20} \mathrm{ClNO}, 253.1$, found 253.1.<smiles>CC(C)(C)NCc1ccccc1F</smiles>

$N$-(2-fluorobenzyl)-2-methylpropan-2-amine (S27):

Prepared according to General Reductive Amination Procedure. 2-fluorobenzaldehyde $(0.42 \mathrm{~mL}$, $4.0 \mathrm{mmol}, 1.0$ equiv) and tert-butyl amine $(0.73 \mathrm{~mL}, 4.8 \mathrm{mmol}, 1.2$ equiv) were stirred in $\mathrm{MeOH}$ $(20 \mathrm{~mL}, 0.2 \mathrm{M})$ for 3 hours. After $\mathrm{NaBH}_{4}(227 \mathrm{mg}, 4.8 \mathrm{mmol}, 1.5$ equiv) was added, the reaction was stirred for an additional 1.5 hours. The title compound was obtained as a colorless oil (508 mg, $70 \%)$.

${ }^{1} \mathbf{H}$ NMR $(600 \mathrm{MHz}, \mathrm{CDCl} 3) \delta 7.39(\mathrm{td}, \mathrm{J}=7.6,1.8 \mathrm{~Hz}, 1 \mathrm{H}), 7.21(\mathrm{tdd}, \mathrm{J}=7.5,5.2,1.8 \mathrm{~Hz}, 1 \mathrm{H})$, 7.09 (td, J = 7.5, 1.2 Hz, 1H), 7.01 (ddd, J = 9.8, 8.2, $1.2 \mathrm{~Hz}, 1 \mathrm{H}), 3.78(\mathrm{~s}, 2 \mathrm{H}), 1.19$ (s, 9H) ppm. ${ }^{13}$ C NMR $(151 \mathrm{MHz}, \mathrm{CDCl} 3) \delta 161.3\left(\mathrm{~d},{ }^{1} \mathrm{~J}_{\mathrm{C}-\mathrm{F}}=245.0 \mathrm{~Hz}\right), 130.6\left(\mathrm{~d},{ }^{3} \mathrm{~J}_{\mathrm{C}-\mathrm{F}}=4.9 \mathrm{~Hz}\right), 128.6(\mathrm{~d}$, $\left.{ }^{3} \mathrm{~J}_{\mathrm{C}-\mathrm{F}}=7.9 \mathrm{~Hz}\right), 128.5,124.3\left(\mathrm{~d},{ }^{4} \mathrm{~J}_{\mathrm{C}-\mathrm{F}}=3.5 \mathrm{~Hz}\right), 115.4\left(\mathrm{~d},{ }^{2} \mathrm{~J}_{\mathrm{C}-\mathrm{F}}=21.9 \mathrm{~Hz}\right), 51.0,40.9\left(\mathrm{~d},{ }^{3} \mathrm{~J}_{\mathrm{C}-\mathrm{F}}=\right.$ $3.5 \mathrm{~Hz}), 29.3 \mathrm{ppm}$.

LRMS (EI) m/z: [M] $]^{+}$calc'd. for $\mathrm{C}_{11} \mathrm{H}_{16} \mathrm{FN}, 181.1$, found 181.2.<smiles>CC(C)(C)N(Cc1ccccc1F)C(=O)CCl</smiles>

$N$-(tert-butyl)-2-chloro- $N$-(2-fluorobenzyl)acetamide (S28):

Prepared according to General Acylation Procedure. $N$-(2-fluorobenzyl)-2-methylpropan-2-amine (S27) $\left(399 \mathrm{mg}, 2.0 \mathrm{mmol}, 1.0\right.$ equiv), $\mathrm{Et}_{3} \mathrm{~N}$ (0.31 mL, $2.2 \mathrm{mmol}, 1.1$ equiv), and chloroacetyl chloride (0.16 mL, $2.0 \mathrm{mmol}, 1.1$ equiv) were stirred in $\mathrm{CH}_{2} \mathrm{Cl}_{2}(20 \mathrm{~mL}, 0.1 \mathrm{M})$ for 3 hours. Purification on silica gel (30\% EtOAc/hexanes) afforded the title compound as a white amorphous solid (473 mg, 92\%).

${ }^{1} \mathbf{H}$ NMR $(600 \mathrm{MHz}, \mathrm{CDCl} 3) \delta 7.29(\mathrm{dtd}, \mathrm{J}=7.4,6.3,5.2,1.8 \mathrm{~Hz}, 1 \mathrm{H}), 7.23(\mathrm{t}, \mathrm{J}=7.2 \mathrm{~Hz}, 1 \mathrm{H})$, $7.21-7.15$ (t, J = 7.2 Hz, 1H), 7.08 (ddd, J = 10.3, 8.2, $1.2 \mathrm{~Hz}, 1 \mathrm{H}), 4.69$ (s, 2H), 3.98 (s, 2H), $1.46(\mathrm{~s}, 9 \mathrm{H}) \mathrm{ppm}$. 
${ }^{13} \mathbf{C}$ NMR $(151 \mathrm{MHz}, \mathrm{CDCl} 3) \delta 167.77,159.79\left(\mathrm{~d},{ }^{1} \mathrm{~J}_{\mathrm{C}-\mathrm{F}}=245.8 \mathrm{~Hz}\right), 129.21\left(\mathrm{~d},{ }^{3} \mathrm{~J}_{\mathrm{C}-\mathrm{F}}=7.9 \mathrm{~Hz}\right)$, $127.06\left(\mathrm{~d},{ }^{3} \mathrm{~J}_{\mathrm{C}-\mathrm{F}}=3.8 \mathrm{~Hz}\right), 125.87\left(\mathrm{~d},{ }^{2} \mathrm{~J}_{\mathrm{C}-\mathrm{F}}=13.9 \mathrm{~Hz}\right), 124.74\left(\mathrm{~d},{ }^{4} \mathrm{~J}_{\mathrm{C}-\mathrm{F}}=3.8 \mathrm{~Hz}\right), 115.80\left(\mathrm{~d},{ }^{2} \mathrm{~J}_{\mathrm{C}-\mathrm{F}}\right.$ $=20.8 \mathrm{~Hz}), 58.89,44.15,42.99\left(\mathrm{~d},{ }^{3} \mathrm{~J}_{\mathrm{C}-\mathrm{F}}=5.9 \mathrm{~Hz}\right), 28.40$.

LRMS (EI) m/z: [M] $]^{+}$calc'd. for $\mathrm{C}_{13} \mathrm{H}_{17} \mathrm{ClFNO}$, 257.1, found 257.1.<smiles>CC(C)(C)NCc1cccc(F)c1</smiles>

\section{$N$-(3-fluorobenzyl)-2-methylpropan-2-amine (S29):}

Prepared according to General Reductive Amination Procedure. 3-Fluorobenzaldehyde $(0.42 \mathrm{~mL}$, $4.0 \mathrm{mmol}, 1.0$ equiv) and tert-butyl amine $(0.50 \mathrm{~mL}, 4.8 \mathrm{mmol}, 1.2$ equiv) were stirred in $\mathrm{MeOH}$ $(20 \mathrm{~mL}, 0.2 \mathrm{M})$ for 3 hours. After $\mathrm{NaBH}_{4}(227 \mathrm{mg}, 6.0 \mathrm{mmol}, 1.5$ equiv) was added, the reaction was stirred for an additional 14 hours. The title compound was obtained as a white amorphous solid (633 mg, 87\%).

${ }^{1}$ H NMR $\left(400 \mathrm{MHz}, \mathrm{CDCl}_{3}\right) \delta 7.30-7.23(\mathrm{~m}, 1 \mathrm{H}), 7.19-7.10(\mathrm{~m}, 2 \mathrm{H}), 6.96-6.88(\mathrm{~m}, 1 \mathrm{H}), 3.75$ (s, 2H), 1.20 (s, 9H) ppm.

${ }^{13} \mathbf{C ~ N M R}\left(151 \mathrm{MHz}, \mathrm{CDCl}_{3}\right) \delta 163.1\left(\mathrm{~d},{ }^{1} \mathrm{~J}_{\mathrm{C}-\mathrm{F}}=245.6 \mathrm{~Hz}\right), 130.0\left(\mathrm{~d},{ }^{3} \mathrm{~J}_{\mathrm{C}-\mathrm{F}}=8.3 \mathrm{~Hz}\right), 124.3,122.4$ $\left(\mathrm{d},{ }^{4} \mathrm{~J}_{\mathrm{C}-\mathrm{F}}=2.9 \mathrm{~Hz}\right), 115.7\left(\mathrm{~d},{ }^{2} \mathrm{~J}_{\mathrm{C}-\mathrm{F}}=21.4 \mathrm{~Hz}\right), 114.1\left(\mathrm{~d},{ }^{2} \mathrm{~J}_{\mathrm{C}-\mathrm{F}}=21.2 \mathrm{~Hz}\right), 64.7,46.7,28.9 \mathrm{ppm}$.

LRMS (EI) m/z: [M] $]^{+}$calc'd. for $\mathrm{C}_{11} \mathrm{H}_{16} \mathrm{FN}, 181.1$, found 181.0.<smiles>CC(C)(C)N(Cc1cccc(F)c1)C(=O)CCl</smiles>

$\boldsymbol{N}$-(tert-butyl)-2-chloro- $\boldsymbol{N}$-(3-fluorobenzyl)acetamide (S30):

Prepared according to General Acylation Procedure. $N$-(3-fluorobenzyl)-2-methylpropan-2-amine (S29) $\left(544 \mathrm{mg}, 3.0 \mathrm{mmol}, 1.0\right.$ equiv), $\mathrm{Et}_{3} \mathrm{~N}$ (0.50 mL, $3.6 \mathrm{mmol}, 1.2$ equiv), and chloroacetyl chloride $\left(0.29 \mathrm{~mL}, 3.6 \mathrm{mmol}, 1.2\right.$ equiv) were stirred in $\mathrm{CH}_{2} \mathrm{Cl}_{2}(30 \mathrm{~mL}, 0.1 \mathrm{M})$ for 2 hours. Purification on silica gel (10-20\% EtOAc/hexanes) afforded the title compound as a white amorphous solid (601 mg, 78\%).

${ }^{1} \mathbf{H}$ NMR $\left(400 \mathrm{MHz}, \mathrm{CDCl}_{3}\right) \delta 7.35(\mathrm{td}, \mathrm{J}=8.0,5.8 \mathrm{~Hz}, 1 \mathrm{H}), 7.03-6.95(\mathrm{~m}, 2 \mathrm{H}), 6.95-6.87(\mathrm{~m}$, $1 \mathrm{H}), 4.65$ (s, 2H), $3.95(\mathrm{~s}, 2 \mathrm{H}), 1.45(\mathrm{~s}, 9 \mathrm{H}) \mathrm{ppm}$.

${ }^{13} \mathrm{C}$ NMR $\left(151 \mathrm{MHz}, \mathrm{CDCl}_{3}\right) \delta 167.7,163.5\left(\mathrm{~d},{ }^{1} \mathrm{~J}_{\mathrm{C}-\mathrm{F}}=247.7 \mathrm{~Hz}\right), 141.7\left(\mathrm{~d},{ }^{3} \mathrm{~J}_{\mathrm{C}-\mathrm{F}}=6.6 \mathrm{~Hz}\right), 130.9$ $\left(\mathrm{d},{ }^{3} \mathrm{~J}_{\mathrm{C}-\mathrm{F}}=8.3 \mathrm{~Hz}\right), 121.1\left(\mathrm{~d},{ }^{4} \mathrm{~J}_{\mathrm{C}-\mathrm{F}}=2.8 \mathrm{~Hz}\right), 114.6\left(\mathrm{~d},{ }^{2} \mathrm{~J}_{\mathrm{C}-\mathrm{F}}=21.1 \mathrm{~Hz}\right), 112.7\left(\mathrm{~d},{ }^{2} \mathrm{~J}_{\mathrm{C}-\mathrm{F}}=22.3 \mathrm{~Hz}\right)$, 59.0, $48.6\left(\mathrm{~d},{ }^{4} \mathrm{~J}_{\mathrm{C}-\mathrm{F}}=2.0 \mathrm{~Hz}\right), 44.2,28.6 \mathrm{ppm}$.

LRMS (EI) m/z: [M] $]^{+}$calc'd. for $\mathrm{C}_{13} \mathrm{H}_{17} \mathrm{ClFNO}$, 257.1, found 257.1.<smiles>CC(C)(C)NCc1ccccc1Cl</smiles>

$N$-(2-chlorobenzyl)-2-methylpropan-2-amine (S31):

Prepared according to General Reductive Amination Procedure. 2-chlorobenzaldehyde $(0.45 \mathrm{~mL}$, $4.0 \mathrm{mmol}, 1.0$ equiv) and tert-butyl amine $(0.73 \mathrm{~mL}, 4.8 \mathrm{mmol}, 1.2$ equiv) were stirred in $\mathrm{MeOH}$ $(20 \mathrm{~mL}, 0.2 \mathrm{M})$ for 3 hours. After $\mathrm{NaBH}_{4}(227 \mathrm{mg}, 6.0 \mathrm{mmol}, 1.5$ equiv) was added, the reaction 
was stirred for an additional 4 hours. The title compound was obtained as a colorless oil (515 mg, $65 \%$ ).

${ }^{1} \mathbf{H}$ NMR $(600 \mathrm{MHz}, \mathrm{CDCl} 3) \delta 7.45(\mathrm{dd}, \mathrm{J}=7.5,1.7 \mathrm{~Hz}, 1 \mathrm{H}), 7.33(\mathrm{dd}, \mathrm{J}=7.9,1.4 \mathrm{~Hz}, 1 \mathrm{H}), 7.22$ $(\mathrm{td}, \mathrm{J}=7.5,1.4 \mathrm{~Hz}, 1 \mathrm{H}), 7.17(\mathrm{td}, \mathrm{J}=7.6,1.8 \mathrm{~Hz}, 1 \mathrm{H}), 3.82(\mathrm{~s}, 2 \mathrm{H}), 1.20$ (s, 9H) ppm.

${ }^{1} \mathrm{H}$ NMR spectrum is consistent with reported values. ${ }^{16}$<smiles>CC(C)(C)N(Cc1ccccc1Cl)C(=O)CCl</smiles>

$\boldsymbol{N}$-(tert-butyl)-2-chloro- $\boldsymbol{N}$-(2-chlorobenzyl)acetamide (S32):

Prepared according to General Acylation Procedure. $N$-(2-chlorobenzyl)-2-methylpropan-2-amine (S31) $\left(395 \mathrm{mg}, 2.0 \mathrm{mmol}, 1.0\right.$ equiv), $\mathrm{Et}_{3} \mathrm{~N}$ (0.31 mL, $2.2 \mathrm{mmol}, 1.1$ equiv), and chloroacetyl chloride (0.18 mL, $2.2 \mathrm{mmol}, 1.1$ equiv) were stirred in $\mathrm{CH}_{2} \mathrm{Cl}_{2}(20 \mathrm{~mL}, 0.1 \mathrm{M})$ for 3.5 hours. Purification on silica gel (10-20\% EtOAc/hexanes) afforded the title compound as a light yellow oil (435 mg, 79\%).

${ }^{1}$ H NMR $(600 \mathrm{MHz}, \mathrm{CDCl} 3) \delta 7.40(\mathrm{~d}, \mathrm{~J}=8.0 \mathrm{~Hz}, 1 \mathrm{H}), 7.32(\mathrm{t}, \mathrm{J}=7.5 \mathrm{~Hz}, 1 \mathrm{H}), 7.29$ - $7.23(\mathrm{~m}$, $2 \mathrm{H}), 4.67$ (s, 2H), $3.92(\mathrm{~s}, 2 \mathrm{H}), 1.46(\mathrm{~s}, 9 \mathrm{H}) \mathrm{ppm}$.

${ }^{13}$ C NMR (151 MHz, CDC13) $\delta 167.8,136.0,132.1,130.1,128.9,127.5,126.8,59.0,47.0,44.1$, $28.4 \mathrm{ppm}$.

LRMS (EI) m/z: [M] ${ }^{+}$calc'd. for $\mathrm{C}_{13} \mathrm{H}_{17} \mathrm{Cl}_{2} \mathrm{NO}$, 273.1, found 273.1.<smiles>CC(C)(C)NCc1cccc(Cl)c1</smiles>

\section{$N$-(3-chlorobenzyl)-2-methylpropan-2-amine (S33):}

Prepared according to General Reductive Amination Procedure. 3-chlorobenzaldehyde $(0.45 \mathrm{~mL}$, $4.0 \mathrm{mmol}, 1.0$ equiv) and tert-butyl amine $(0.73 \mathrm{~mL}, 4.8 \mathrm{mmol}, 1.2$ equiv) were stirred in $\mathrm{MeOH}$ $(20 \mathrm{~mL}, 0.2 \mathrm{M})$ for 4.5 hours. After $\mathrm{NaBH}_{4}(227 \mathrm{mg}, 6.0 \mathrm{mmol}, 1.5$ equiv) was added, the reaction was stirred for an additional 1.5 hours. The title compound was obtained as a light-yellow oil (744 mg, 94\%).

${ }^{1}$ H NMR (400 MHz, CDCl3) $\delta 7.36(\mathrm{~s}, 1 \mathrm{H}), 7.24-7.14(\mathrm{~m}, 3 \mathrm{H}), 3.70$ (s, 2H), 1.17 (s, 9H) ppm. ${ }^{1} \mathrm{H}$ NMR spectrum is consistent with reported values. ${ }^{17}$<smiles>CCCN(Cc1cccc(Cl)c1)C(=O)CCl</smiles>

$\boldsymbol{N}$-(tert-butyl)-2-chloro- $\boldsymbol{N}$-(3-chlorobenzyl)acetamide (S34):

Prepared according to General Acylation Procedure. $N$-(3-chlorobenzyl)-2-methylpropan-2-amine (S33) $(395 \mathrm{mg}, 2.0 \mathrm{mmol}, 1.0$ equiv), Et $3 \mathrm{~N}$ (0.15 mL, $2.2 \mathrm{mmol}, 1.1$ equiv), and chloroacetyl

\footnotetext{
${ }^{16}$ Franchi, P.; Casati, C.; Mezzina, E.; Lucarini, M. Kinetic control of the direction of inclusion of nitroxide cyclodextrines. Org. Biomol. Chem., 2011, 9, 6396-6401.

${ }^{17}$ Jiang, G.; Chen, J.; Huang, J-S.; Che, C-M. Highly efficient oxidation of amines to imines by singlet oxygen and its application in ugi-type reactions. Org. Lett., 2009, 11, 4568-4571.
} 
chloride (0.18 mL, $2.2 \mathrm{mmol}, 1.1$ equiv) were stirred in $\mathrm{CH}_{2} \mathrm{Cl}_{2}(20 \mathrm{~mL}, 0.1 \mathrm{M})$ for 3.5 hours, which afforded the title compound as a white amorphous solid (436 mg, 72\%).

${ }^{1} \mathbf{H}$ NMR $(400 \mathrm{MHz}, \mathrm{CDCl} 3) \delta 7.36-7.23(\mathrm{~m}, 2 \mathrm{H}), 7.19(\mathrm{~s}, 1 \mathrm{H}), 7.09(\mathrm{~d}, \mathrm{~J}=7.8 \mathrm{~Hz}, 1 \mathrm{H}), 4.64$ (s, 2H), 3.95 (s, 2H), 1.45 (s, 9H) ppm.

${ }^{13}$ C NMR (101 MHz, CDCl3) $\delta$ 167.6, 140.9, 135.2, 130.4, 127.8, 125.7, 123.5, 58.9, 48.4, 44.0, $28.4 \mathrm{ppm}$.

LRMS (EI) m/z: [M] ${ }^{+}$calc'd. for $\mathrm{C}_{13} \mathrm{H}_{17} \mathrm{Cl}_{2} \mathrm{NO}$, 273.1, found 273.1.<smiles>CC(C)(C)NCc1ccc(Cl)cc1</smiles>

$N$-(4-chlorobenzyl)-2-methylpropan-2-amine (S35):

Prepared according to General Reductive Amination Procedure. 4-Chlorobenzaldehyde (562 mg, $4.0 \mathrm{mmol}, 1.0$ equiv) and tert-butyl amine $(0.50 \mathrm{~mL}, 4.8 \mathrm{mmol}, 1.2$ equiv) were stirred in $\mathrm{MeOH}$ $(20 \mathrm{~mL}, 0.2 \mathrm{M})$ for 17 hours. After $\mathrm{NaBH}_{4}(227 \mathrm{mg}, 6.0 \mathrm{mmol}, 1.5$ equiv) was added, the reaction was stirred for an additional $30 \mathrm{~min}$. The title compound was obtained as a white amorphous solid (676 mg, 85\%).

${ }^{1} \mathbf{H}$ NMR $\left(600 \mathrm{MHz}, \mathrm{CDCl}_{3}\right) \delta 7.29-7.26(\mathrm{~m}, 4 \mathrm{H}), 3.70(\mathrm{~s}, 2 \mathrm{H}), 1.17(\mathrm{~s}, 9 \mathrm{H}) \mathrm{ppm}$.

${ }^{13}$ C NMR $\left(151 \mathrm{MHz}, \mathrm{CDCl}_{3}\right) \delta 140.2,132.6,129.8,128.6,51.0,46.7,29.3$ ppm.

LRMS (EI) m/z: [M] ${ }^{+}$calc'd. for $\mathrm{C}_{11} \mathrm{H}_{16} \mathrm{ClN}$, 197.1, found 197.1.<smiles>CC(C)(C)N(Cc1ccc(Cl)cc1)C(=O)CCl</smiles>

$N$-(tert-butyl)-2-chloro- $\boldsymbol{N}$-(4-chlorobenzyl)acetamide (S36):

Prepared according to General Acylation Procedure. $N$-(4-chlorobenzyl)-2-methylpropan-2-amine (S35) $\left(593 \mathrm{mg}, 3.0 \mathrm{mmol}, 1.0\right.$ equiv), $\mathrm{Et}_{3} \mathrm{~N}$ (0.50 mL, $3.6 \mathrm{mmol}, 1.2$ equiv), and chloroacetyl chloride $\left(0.29 \mathrm{~mL}, 3.6 \mathrm{mmol}, 1.2\right.$ equiv) were stirred in $\mathrm{CH}_{2} \mathrm{Cl}_{2}(30 \mathrm{~mL}, 0.1 \mathrm{M})$ for 1 hour. The title compound was obtained as a yellow oil $(823 \mathrm{mg}, 100 \%)$.

${ }^{1} \mathbf{H}$ NMR $\left(400 \mathrm{MHz}, \mathrm{CDCl}_{3}\right) \delta 7.38(\mathrm{~d}, \mathrm{~J}=8.5 \mathrm{~Hz}, 2 \mathrm{H}), 7.17(\mathrm{~d}, \mathrm{~J}=8.7 \mathrm{~Hz}, 2 \mathrm{H}), 4.65(\mathrm{~s}, 2 \mathrm{H}), 3.97$ (s, 2H), 1.46 (s, 9H) ppm.

${ }^{1} \mathrm{H}$ NMR spectrum is consistent with reported values. ${ }^{18}$<smiles>COc1ccc(F)c(CNC(C)(C)C)c1</smiles>

$N$-(2-fluoro-5-methoxybenzyl)-2-methylpropan-2-amine (S37):

Prepared according to General Reductive Amination Procedure. 2-fluoro-5-methoxybenzaldehyde $(0.25 \mathrm{~mL}, 2.0 \mathrm{mmol}, 1.0$ equiv) and tert-butyl amine $(0.41 \mathrm{~mL}, 2.4 \mathrm{mmol}, 1.2$ equiv) were stirred in $\mathrm{MeOH}(4 \mathrm{~mL}, 0.5 \mathrm{M})$ for 14 hours. After $\mathrm{NaBH}_{4}(114 \mathrm{mg}, 3.0 \mathrm{mmol}, 1.5$ equiv) was added, the

\footnotetext{
${ }^{18}$ Pedroni, J.; Boghi, M.; Saget, T.; Cramer, N. Access to $\beta$-Lactams by Enantioselective Palladium(0)-Catalyzed $\mathrm{C}\left(\mathrm{sp}^{3}\right)$-H Alkylation. Angew. Chem. Int. Ed. 2014, 53, 9064-9067.
} 
reaction was stirred for an additional 1 hour. The title compound was obtained as a light-yellow oil (386 mg, 46\%).

${ }^{1}$ H NMR $(400 \mathrm{MHz}, \mathrm{CDCl} 3) \delta 6.95-6.86(\mathrm{~m}, 2 \mathrm{H}), 6.69(\mathrm{ddd}, \mathrm{J}=8.9,4.0,3.2 \mathrm{~Hz}, 1 \mathrm{H}), 3.75(\mathrm{~s}$, $3 \mathrm{H}), 3.72(\mathrm{~s}, 2 \mathrm{H}), 1.17$ (s, 9H) ppm.

${ }^{13} \mathbf{C}$ NMR $(101 \mathrm{MHz}, \mathrm{CDCl} 3) \delta 155.8\left(\mathrm{~d},{ }^{4} \mathrm{~J}_{\mathrm{C}-\mathrm{F}}=2.0 \mathrm{~Hz}\right), 155.5\left(\mathrm{~d},{ }^{1} \mathrm{~J}_{\mathrm{C}-\mathrm{F}}=237.4 \mathrm{~Hz}\right), 129.1(\mathrm{~d}$, $\left.{ }^{2} \mathrm{~J}_{\mathrm{C}-\mathrm{F}}=16.8 \mathrm{~Hz}\right), 115.7\left(\mathrm{~d},{ }^{2} \mathrm{~J}_{\mathrm{C}-\mathrm{F}}=23.8 \mathrm{~Hz}\right), 115.4\left(\mathrm{~d},{ }^{3} \mathrm{~J}_{\mathrm{C}-\mathrm{F}}=4.7 \mathrm{~Hz}\right), 113.2\left(\mathrm{~d},{ }^{3} \mathrm{~J}_{\mathrm{C}-\mathrm{F}}=8.2 \mathrm{~Hz}\right)$, $55.8,50.9,41.0\left(\mathrm{~d},{ }^{3} \mathrm{~J}_{\mathrm{C}-\mathrm{F}}=2.9 \mathrm{~Hz}\right), 29.1 \mathrm{ppm}$.

LRMS (EI) m/z: [M] $]^{+}$calc'd. for $\mathrm{C}_{12} \mathrm{H}_{18} \mathrm{FNO}, 211.1$, found 211.2.<smiles>COc1ccc(F)c(CN(C)C(=O)CCl)c1</smiles>

$N$-(tert-butyl)-2-chloro- $N$-(2-fluoro-5-methoxybenzyl)acetamide (S38):

Prepared according to General Acylation Procedure. N-(2-fluoro-5-methoxybenzyl)-2methylpropan-2-amine (S37) (211 mg, $1.0 \mathrm{mmol}, 1.0$ equiv), $\mathrm{Et}_{3} \mathrm{~N}(0.15 \mathrm{~mL}, 1.1 \mathrm{mmol}, 1.1$ equiv), and chloroacetyl chloride $\left(0.09 \mathrm{~mL}, 1.1 \mathrm{mmol}, 1.1\right.$ equiv) were stirred in $\mathrm{CH}_{2} \mathrm{Cl}_{2}(10 \mathrm{~mL}, 0.1 \mathrm{M})$ for 4.5 hours, which afforded the title compound as a tan amorphous solid $(263 \mathrm{mg}, 91 \%)$.

${ }^{1} \mathbf{H}$ NMR 1H NMR $(400 \mathrm{MHz}, \mathrm{CDCl} 3) \delta 6.99(\mathrm{t}, \mathrm{J}=9.1 \mathrm{~Hz}, 1 \mathrm{H}), 6.80-6.72(\mathrm{~m}, 1 \mathrm{H}), 6.76-6.70$ $(\mathrm{m}, 1 \mathrm{H}), 4.64(\mathrm{~s}, 2 \mathrm{H}), 3.98(\mathrm{~s}, 2 \mathrm{H}), 3.76(\mathrm{~s}, 3 \mathrm{H}), 1.46(\mathrm{~s}, 9 \mathrm{H}) \mathrm{ppm}$.

${ }^{13} \mathbf{C}$ NMR $(101 \mathrm{MHz}, \mathrm{CDCl} 3) \delta 167.8,156.4\left(\mathrm{~d},{ }^{4} \mathrm{~J}_{\mathrm{C}-\mathrm{F}}=2.0 \mathrm{~Hz}\right), 154.0\left(\mathrm{~d},{ }^{1} \mathrm{~J}_{\mathrm{C}-\mathrm{F}}=238.2 \mathrm{~Hz}\right), 126.8$ $\left(\mathrm{d},{ }^{2} \mathrm{~J}_{\mathrm{C}-\mathrm{F}}=15.7 \mathrm{~Hz}\right), 116.4\left(\mathrm{~d},{ }^{2} \mathrm{~J}_{\mathrm{C}-\mathrm{F}}=22.9 \mathrm{~Hz}\right), 113.3\left(\mathrm{~d},{ }^{3} \mathrm{~J}_{\mathrm{C}-\mathrm{F}}=7.9 \mathrm{~Hz}\right), 112.6\left(\mathrm{~d},{ }^{3} \mathrm{~J}_{\mathrm{C}-\mathrm{F}}=3.8 \mathrm{~Hz}\right)$, $58.9,55.9,44.1,43.2\left(\mathrm{~d},{ }^{3} \mathrm{~J}_{\mathrm{C}-\mathrm{F}}=5.1 \mathrm{~Hz}\right), 28.4 \mathrm{ppm}$.

LRMS (EI) m/z: [M] $]^{+}$calc'd. for $\mathrm{C}_{14} \mathrm{H}_{19} \mathrm{ClFNO}_{2}$, 287.1, found 287.1.<smiles>CN(Cc1ccccc1)C(=O)CCl</smiles>

$N$-benzyl- $N$-(tert-butyl)-2-chloroacetamide (S39):

Prepared according to General Acylation Procedure. $N$-benzyl-2-methylpropan-2-amine (S23) (688 mg, $4.2 \mathrm{mmol}, 1.0$ equiv), $\mathrm{Et}_{3} \mathrm{~N}(0.64 \mathrm{~mL}, 4.6 \mathrm{mmol}, 1.1$ equiv), and chloroacetyl chloride (0.37 mL, $4.6 \mathrm{mmol}, 1.1$ equiv) were stirred in $\mathrm{CH}_{2} \mathrm{Cl}_{2}(42 \mathrm{~mL}, 0.1 \mathrm{M})$ for 3.5 hours. The title compound was afforded as a white amorphous solid $(946 \mathrm{mg}, 99 \%)$.

${ }^{1}$ H NMR $(400 \mathrm{MHz}, \mathrm{cdcl} 3) \delta 7.38(\mathrm{t}, \mathrm{J}=7.4 \mathrm{~Hz}, 2 \mathrm{H}), 7.29(\mathrm{t}, \mathrm{J}=7.3 \mathrm{~Hz}, 1 \mathrm{H}), 7.20(\mathrm{~d}, \mathrm{~J}=6.9 \mathrm{~Hz}$, $2 \mathrm{H}), 4.66(\mathrm{~s}, 2 \mathrm{H}), 3.98(\mathrm{~s}, 2 \mathrm{H}), 1.46(\mathrm{~s}, 9 \mathrm{H}) \mathrm{ppm}$.

${ }^{1} \mathrm{H}$ NMR spectrum is consistent with reported values. ${ }^{19}$

\footnotetext{
${ }^{19}$ Pedroni, J.; Boghi, M.; Saget, T.; Cramer, N. Access to $\beta$-Lactams by Enantioselective Palladium(0)-Catalyzed $\mathrm{C}\left(\mathrm{sp}^{3}\right)$-H Alkylation. Angew. Chem. Int. Ed. 2014, 126, 9210-9213.
} 
<smiles>CC(C)(NCc1ccccc1)c1ccccc1</smiles>

\section{$N$-benzyl-2-phenylpropan-2-amine (S40):}

Prepared according to General Reductive Amination Procedure. Benzaldehyde $(0.20 \mathrm{~mL}, 2.0$ mmol, 1.0 equiv) and 2-phenylpropan-2-amine $(0.35 \mathrm{~mL}, 2.4 \mathrm{mmol}, 1.2$ equiv) were stirred in $\mathrm{MeOH}\left(10 \mathrm{~mL}, 0.2 \mathrm{M}\right.$ ) for 14 hours. After $\mathrm{NaBH}_{4}(114 \mathrm{mg}, 3.0 \mathrm{mmol}, 1.5$ equiv) was added, the reaction was stirred for an additional 2 hours. The title compound was obtained as a colorless oil (414 mg, 92\%).

${ }^{1} \mathbf{H}$ NMR $(600 \mathrm{MHz}, \mathrm{CDCl} 3) \delta 7.49(\mathrm{~d}, \mathrm{~J}=7.7 \mathrm{~Hz}, 2 \mathrm{H}), 7.32(\mathrm{t}, \mathrm{J}=7.6 \mathrm{~Hz}, 2 \mathrm{H}), 7.28-7.22(\mathrm{~m}$, $4 \mathrm{H}), 7.21-7.15(\mathrm{~m}, 2 \mathrm{H}), 3.44(\mathrm{~s}, 2 \mathrm{H}), 1.49$ (s, 6H) ppm.

${ }^{1} \mathrm{H}$ NMR spectrum is consistent with reported values. ${ }^{20}$<smiles>CC(C)(c1ccccc1)N(Cc1ccccc1)C(=O)CCl</smiles>

\section{$N$-benzyl-2-chloro- $N$-(2-phenylpropan-2-yl)acetamide (S41):}

Prepared according to General Acylation Procedure. $N$-benzyl-2-phenylpropan-2-amine (S40) (225 mg, $1.0 \mathrm{mmol}, 1.0$ equiv), $\mathrm{Et}_{3} \mathrm{~N}$ ( $0.15 \mathrm{~mL}, 1.1 \mathrm{mmol}, 1.1$ equiv), and chloroacetyl chloride ( $0.09 \mathrm{~mL}, 1.1 \mathrm{mmol}, 1.1$ equiv) were stirred in $\mathrm{CH}_{2} \mathrm{Cl}_{2}(10 \mathrm{~mL}, 0.1 \mathrm{M})$ for 16 hours. Purification on silica gel (10\% EtOAc/hexanes) afforded the title compound as a white amorphous solid (138 $\mathrm{mg}, 46 \%)$.

${ }^{1} \mathbf{H}$ NMR $(600 \mathrm{MHz}, \mathrm{CDCl} 3) \delta 7.40(\mathrm{t}, \mathrm{J}=7.5 \mathrm{~Hz}, 2 \mathrm{H}), 7.32$ (dd, J = 9.4, $\left.5.9 \mathrm{~Hz}, 7 \mathrm{H}\right), 7.22$ (hept, $\mathrm{J}=4.2 \mathrm{~Hz}, 1 \mathrm{H}), 4.92(\mathrm{~s}, 2 \mathrm{H}), 3.89(\mathrm{~s}, 2 \mathrm{H}), 1.70(\mathrm{~s}, 6 \mathrm{H}) \mathrm{ppm}$.

${ }^{13}$ C NMR (151 MHz, CDCl3) $\delta 167.9,148.1,138.7,129.2,128.7,127.7,126.8,126.4,124.4$, 63.1, 49.5, 43.7, 29.4 ppm.

LRMS (EI) m/z: [M] ${ }^{+}$calc'd. for $\mathrm{C}_{18} \mathrm{H}_{20} \mathrm{ClNO}, 301.1$, found 301.1.<smiles>CNCc1ccccc1</smiles>

\section{$N$-methyl-1-phenylmethanamine (S42):}

Prepared according to General Reductive Amination Procedure. Benzaldehyde $(0.41 \mathrm{~mL}, 4.0$ mmol, 1.0 equiv) and methanamine $(0.37 \mathrm{~mL}, 4.8 \mathrm{mmol}, 1.2$ equiv) were stirred in $\mathrm{MeOH}(20 \mathrm{~mL}$, $0.2 \mathrm{M})$ for 2 hours. After $\mathrm{NaBH}_{4}(227 \mathrm{mg}, 6.0 \mathrm{mmol}, 1.5$ equiv) was added, the reaction was stirred for an additional $30 \mathrm{~min}$. The title compound was obtained as a white amorphous solid (479 $\mathrm{mg}$, $99 \%$ ).

${ }^{1}$ H NMR (400 MHz, CDCl3) $\delta 7.36-7.30(\mathrm{~m}, 4 \mathrm{H}), 7.29-7.22(\mathrm{~m}, 1 \mathrm{H}), 3.75(\mathrm{~s}, 2 \mathrm{H}), 2.46$ (s, $3 \mathrm{H}), 1.59$ (s, 1H) ppm.

\footnotetext{
${ }^{20}$ Milburn, R. R.; Snieckus, V. ortho-Anisylsufonyl as a protecting group for secondary amines: mild $\mathrm{Ni}^{0}$-catalyzed hydrodesulfonylation. Angew. Chem. Int. Ed. 2004, 43, 892-893.
} 
${ }^{1} \mathrm{H}$ NMR spectrum is consistent with reported values. ${ }^{21}$<smiles>O=C(CCl)N(Cc1ccccc1)C(=O)CCl</smiles>

$N$-benzyl-2-chloro- $N$-methylacetamide (S43):

Prepared according to General Acylation Procedure. N-methyl-1-phenylmethanamine (S42) (364 $\mathrm{mg}, 3.0 \mathrm{mmol}, 1.0$ equiv), $\mathrm{Et}_{3} \mathrm{~N}$ (0.46 mL, $3.3 \mathrm{mmol}, 1.1$ equiv), and chloroacetyl chloride (0.26 $\mathrm{mL}, 1.1 \mathrm{mmol}, 1.1$ equiv) were stirred in $\mathrm{CH}_{2} \mathrm{Cl}_{2}(30 \mathrm{~mL}, 0.1 \mathrm{M})$ for $30 \mathrm{~min}$. Purification on silica gel (10-30\% EtOAc/hexanes) afforded the title compound as a colorless oil (401 $\mathrm{mg}, 68 \%$ ).

${ }^{1}$ H NMR $(600 \mathrm{MHz}, \mathrm{CDCl} 3) \delta 7.40-7.26(\mathrm{~m}, 3 \mathrm{H}), 7.26-7.24(\mathrm{~m}, 1 \mathrm{H}), 7.19(\mathrm{~d}, \mathrm{~J}=7.5 \mathrm{~Hz}, 1 \mathrm{H})$, $4.60(\mathrm{~s}, 2 \mathrm{H}), 4.12(\mathrm{~d}, \mathrm{~J}=22.3 \mathrm{~Hz}, 2 \mathrm{H}), 2.99(\mathrm{~d}, \mathrm{~J}=16.5 \mathrm{~Hz}, 3 \mathrm{H}) \mathrm{ppm}$.

${ }^{1} \mathrm{H}$ NMR spectrum is consistent with reported values. ${ }^{22}$<smiles>CC(C)NCc1ccccc1</smiles>

\section{$N$-benzylpropan-2-amine (S44):}

Prepared according to General Reductive Amination Procedure. Benzaldehyde $(0.41 \mathrm{~mL}, 4.0$ mmol, 1.0 equiv) and propan-2-amine $(0.39 \mathrm{~mL}, 4.8 \mathrm{mmol}, 1.2$ equiv) were stirred in $\mathrm{MeOH}(20$ $\mathrm{mL}, 0.2 \mathrm{M})$ for 2 hours. After $\mathrm{NaBH}_{4}(227 \mathrm{mg}, 6.0 \mathrm{mmol}, 1.5$ equiv) was added, the reaction was stirred for an additional $30 \mathrm{~min}$. The title compound was obtained as a yellow amorphous solid (557 mg, 93\%).

${ }^{1} \mathbf{H}$ NMR $(400 \mathrm{MHz}, \mathrm{CDCl} 3) \delta 7.32(\mathrm{~d}, \mathrm{~J}=4.4 \mathrm{~Hz}, 4 \mathrm{H}), 7.26-7.22(\mathrm{~m}, 1 \mathrm{H}), 3.78(\mathrm{~s}, 2 \mathrm{H}), 2.85$ $(\mathrm{h}, \mathrm{J}=6.2 \mathrm{~Hz}, 1 \mathrm{H}), 1.10(\mathrm{~d}, \mathrm{~J}=6.2 \mathrm{~Hz}, 6 \mathrm{H}) \mathrm{ppm}$.

${ }^{1} \mathrm{H}$ NMR spectrum is consistent with reported values. ${ }^{23}$<smiles>CC(C)N(Cc1ccccc1)C(=O)CCl</smiles>

\section{$N$-benzyl-2-chloro- $N$-isopropylacetamide (S45):}

Prepared according to General Acylation Procedure. $N$-benzylpropan-2-amine (S44) (448 mg, 3.0 mmol, 1.0 equiv), $\mathrm{Et}_{3} \mathrm{~N}$ (0.46 mL, $3.3 \mathrm{mmol}, 1.1$ equiv), and chloroacetyl chloride $(0.26 \mathrm{~mL}, 3.3$ mmol, 1.1 equiv) were stirred in $\mathrm{CH}_{2} \mathrm{Cl}_{2}(30 \mathrm{~mL}, 0.1 \mathrm{M})$ for $30 \mathrm{~min}$. Purification on silica gel (10$30 \%$ EtOAc/hexanes) afforded the title compound as a colorless oil (414 $\mathrm{mg}, 61 \%)$. Mixture of rotamers $\left(\mathrm{H}^{*}\right.$ denotes major rotamer and $\mathrm{H}$ denotes minor rotamer).

\footnotetext{
${ }^{21}$ Ji, P.; Manna, K.; Lin, Z.; Feng, X.; Urban, A.; Song, Y.; Lin, W. Single-site cobalt catalysts at new $\mathrm{Zr}_{12}\left(\mu_{3^{-}}\right.$ $\mathrm{O})_{8}\left(\mu_{3}-\mathrm{OH}\right)_{8}\left(\mu_{2}-\mathrm{OH}\right)_{6}$ metal-organic framework nodes for highly active e hydrogenation of nitroarenes, nitriles and isocyanides. J. Am. Chem. Soc. 2017, 139, 7004-7011.

${ }^{22}$ Pedroni, J.; Boghi, M.; Saget, T.; Cramer, N. Access to $\beta$-Lactams by Enantioselective Palladium(0)-Catalyzed $\mathrm{C}\left(\mathrm{sp}^{3}\right)-\mathrm{H}$ Alkylation. Angew. Chem. Int. Ed. 2014, 126, 9210-9213.

${ }^{23}$ Rauser, M.; Eckert, R.; Gerbershagen, M.; Niggemann, M. Catalyst-free reductive coupling of aromatic and aliphatic nitro compounds with organohalides. Angew. Chem. Int. Ed. 2019, 58, 6713-6717.
} 
${ }^{1} \mathbf{H}$ NMR $(600 \mathrm{MHz}, \mathrm{CDCl} 3) \delta 7.36\left(\mathrm{t}, \mathrm{J}=7.6 \mathrm{~Hz}, 2\left(\mathrm{H}+\mathrm{H}^{*}\right)\right), 7.29\left(\mathrm{t}, \mathrm{J}=7.7 \mathrm{~Hz}, 4\left(\mathrm{H}+\mathrm{H}^{*}\right)\right), 7.22$ $\left(\mathrm{t}, \mathrm{J}=8.1 \mathrm{~Hz}, 4\left(\mathrm{H}+\mathrm{H}^{*}\right)\right), 4.79\left(\mathrm{spt}, \mathrm{J}=6.8 \mathrm{~Hz}, 1 \mathrm{H}^{*}\right), 4.54\left(\mathrm{~m}, 4\left(\mathrm{H}+\mathrm{H}^{*}\right)\right), 4.22(\mathrm{spt}, \mathrm{J}=6.8 \mathrm{~Hz}$, $1 \mathrm{H}), 4.20\left(\mathrm{~s}, 2 \mathrm{H}^{*}\right), 3.91(\mathrm{~s}, 2 \mathrm{H}), 1.20(\mathrm{~d}, \mathrm{~J}=6.6 \mathrm{~Hz}, 3 \mathrm{H}), 1.15\left(\mathrm{~d}, \mathrm{~J}=6.8 \mathrm{~Hz}, 3 \mathrm{H}^{*}\right) \mathrm{ppm}$.

${ }^{1} \mathrm{H}$ NMR spectrum is consistent with reported values. ${ }^{22}$<smiles>O=C(CCl)N(Br)Cc1ccccc1</smiles>

$N, N$-dibenzyl-2-chloroacetamide (S46):

Prepared according to General Acylation Procedure. Dibenzylamine $(0.22 \mathrm{~mL}, 2.0 \mathrm{mmol}, 1.0$ equiv), $\mathrm{Et}_{3} \mathrm{~N}$ (0.31 mL, $2.2 \mathrm{mmol}, 1.1$ equiv), and chloroacetyl chloride $(0.18 \mathrm{~mL}, 2.2 \mathrm{mmol}, 1.1$ equiv) were stirred in $\mathrm{CH}_{2} \mathrm{Cl}_{2}(20 \mathrm{~mL}, 0.1 \mathrm{M})$ for $20 \mathrm{~min}$. Purification on silica gel (10-30\% EtOAc/hexanes) afforded the title compound as a colorless oil. (307 mg, 56\%).

${ }^{1} \mathbf{H}$ NMR $\left(400 \mathrm{MHz}, \mathrm{CDCl}_{3}\right) \delta 7.42-7.27(\mathrm{~m}, 6 \mathrm{H}), 7.23(\mathrm{~d}, \mathrm{~J}=6.4 \mathrm{~Hz}, 2 \mathrm{H}), 7.18-7.15(\mathrm{~d}, \mathrm{~J}=$ $6.4 \mathrm{~Hz}, 2 \mathrm{H}), 4.62(\mathrm{~s}, 2 \mathrm{H}), 4.52(\mathrm{~s}, 2 \mathrm{H}), 4.15(\mathrm{~s}, 2 \mathrm{H})$.

${ }^{1} \mathrm{H}$ NMR spectrum is consistent with reported values. ${ }^{24}$<smiles>CC(C)(C)c1cccc(CNCc2cccc(C(F)(F)F)c2)c1</smiles>

$N$-(3-(tert-butyl)benzyl)-1-(3-(trifluoromethyl)phenyl)methanamine (S47):

3-(trifluoromethyl)benzylamine $(0.29 \mathrm{~mL}, 2.0 \mathrm{mmol}, 1.0$ equiv) was dissolved in DCM ( $8 \mathrm{~mL}$, $0.25 \mathrm{M})$. 3-tert-butylbenzaldehyde (324 mg, $2.0 \mathrm{mmol}, 1.0$ equiv), AcOH (0.11 mL, $2.0 \mathrm{mmol}, 1.0$ equiv), and sodium triacetoxyborohydride $(593 \mathrm{mg}, 2.8 \mathrm{mmol}, 1.4$ equiv) were added and reaction was stirred for 16 hours. $\mathrm{NaBH}_{4}(76 \mathrm{mg}, 2.0 \mathrm{mmol}, 1.0$ equiv) was added and the reaction was stirred for an additional 4 hours. The reaction was quenched with $1 \mathrm{M} \mathrm{NaOH}(\mathrm{aq})$, extracted with DCM (3x), washed with brine, dried over $\mathrm{MgSO}_{4}$, and concentrated under reduced pressure. Purification on silica gel (50\% EtOAc/hexanes) afforded the title compound as a colorless oil (361 $\mathrm{mg}, 56 \%)$.

${ }^{1}$ H NMR $\left(600 \mathrm{MHz}, \mathrm{CDCl}_{3}\right) \delta 7.64(\mathrm{~s}, 1 \mathrm{H}), 7.55(\mathrm{~d}, \mathrm{~J}=7.6 \mathrm{~Hz}, 1 \mathrm{H}), 7.52(\mathrm{~d}, \mathrm{~J}=7.7 \mathrm{~Hz}, 1 \mathrm{H}), 7.44$ $(\mathrm{t}, \mathrm{J}=7.7 \mathrm{~Hz}, 1 \mathrm{H}), 7.35(\mathrm{~s}, 1 \mathrm{H}), 7.32-7.27(\mathrm{~m}, 2 \mathrm{H}), 7.16(\mathrm{dt}, \mathrm{J}=7.0,1.7 \mathrm{~Hz}, 1 \mathrm{H}), 3.88(\mathrm{~s}, 2 \mathrm{H})$, $3.81(\mathrm{~s}, 2 \mathrm{H}), 1.33$ (s, 9H) ppm.

${ }^{13} \mathbf{C ~ N M R}\left(151 \mathrm{MHz}, \mathrm{CDCl}_{3}\right) \delta 151.6,141.5,139.7,131.7,130.9\left(\mathrm{q},{ }^{2} \mathrm{~J}_{\mathrm{C}-\mathrm{F}}=32.0 \mathrm{~Hz}\right), 129.0,128.4$, $125.5,125.3,125.3\left(\mathrm{q},{ }^{1} \mathrm{~J}_{\mathrm{C}-\mathrm{F}}=272.0 \mathrm{~Hz}\right), 125.1\left(\mathrm{q},{ }^{3} \mathrm{~J}_{\mathrm{C}-\mathrm{F}}=3.8 \mathrm{~Hz}\right), 124.3,124.0\left(\mathrm{q},{ }^{3} \mathrm{~J}_{\mathrm{C}-\mathrm{F}}=3.8 \mathrm{~Hz}\right)$, 53.6, 52.7, 34.9, $31.6 \mathrm{ppm}$.

LRMS (EI) m/z: [M] $]^{+}$calc'd. for $\mathrm{C}_{19} \mathrm{H}_{22} \mathrm{~F}_{3} \mathrm{~N}, 321.2$, found 321.3 .<smiles>O=C(CCl)N(Cc1cccc(Br)c1)Cc1cccc(C(F)(F)F)c1</smiles>

${ }^{24}$ Pedroni, J.; Boghi, M.; Saget, T.; Cramer, N. Access to $\beta$-Lactams by Enantioselective Palladium(0)-Catalyzed $\mathrm{C}\left(\mathrm{sp}^{3}\right)$-H Alkylation. Angew. Chem. Int. Ed. 2014, 126, 9210-9213. 


\section{$N$-(3-(tert-butyl)benzyl)-2-chloro- $N$-(3-(trifluoromethyl)benzyl)acetamide (S48):}

Prepared according to General Acylation Procedure. N-(3-(tert-butyl)benzyl)-1-(3(trifluoromethyl)phenyl)methanamine (S47) $\left(321 \mathrm{mg}, 1.0 \mathrm{mmol}, 1.0\right.$ equiv), $\mathrm{Et}_{3} \mathrm{~N}$ (0.17 mL, 1.2 mmol, 1.2 equiv), and chloroacetyl chloride $\left(0.10 \mathrm{~mL}, 1.2 \mathrm{mmol}, 1.2\right.$ equiv) were stirred in $\mathrm{CH}_{2} \mathrm{Cl}_{2}$ $(10 \mathrm{~mL}, 0.1 \mathrm{M})$ for 16 hours. The title compound was obtained as a colorless oil (390 mg, 98\%). Mixture of rotamers $\left(*\right.$ denotes major rotamer ${ }^{\#}$ denotes minor isomer).

${ }^{1} \mathbf{H}$ NMR $\left(600 \mathrm{MHz}, \mathrm{CDCl}_{3}\right) \delta 7.62-7.27^{(*+\#)}(\mathrm{m}, 6 \mathrm{H}), 7.16^{(*+\#)}(\mathrm{d}, \mathrm{J}=27.3 \mathrm{~Hz}, 1 \mathrm{H}), 7.00^{(*+\#)}$ $(\mathrm{dd}, \mathrm{J}=23.3,7.5 \mathrm{~Hz}, 1 \mathrm{H}), 4.65^{*}(\mathrm{~s}, 1 \mathrm{H}), 4.61^{\#}(\mathrm{~s}, 1 \mathrm{H}), 4.58^{\#}(\mathrm{~s}, 1 \mathrm{H}), 4.54^{*}(\mathrm{~s}, 1 \mathrm{H}), 4.18^{*}(\mathrm{~s}, 1 \mathrm{H})$, $4.13^{\#}(\mathrm{~s}, 1 \mathrm{H}), 1.30^{*}(\mathrm{~s}, 9 \mathrm{H}), 1.29^{\#}(\mathrm{~s}, 9 \mathrm{H}) \mathrm{ppm}$.

${ }^{13}$ C NMR $\left(151 \mathrm{MHz}, \mathrm{CDCl}_{3}\right) \delta 167.7^{*}, 167.4^{\#}, 152.6^{*}, 152.1^{\#}, 137.8^{*}, 137.2^{\#}, 135.7^{\#}, 135.0^{*}$, $131.7^{*}, 131.3^{\#}, 130.0^{\#}, 129.8^{\#}, 129.5^{*}, 129.1^{*}, 128.8^{\#}, 125.5^{\#}, 125.4^{*}, 125.4^{\#}, 125.1(\mathrm{~m}), 124.67$ $\left(\mathrm{q},{ }^{3} \mathrm{~J}_{\mathrm{C}-\mathrm{F}}=3.8 \mathrm{~Hz}\right), 123.9^{*}, 123.7^{*}, 51.4^{*}, 50.2^{\#}, 49.1^{\#}, 48.6^{*}, 41.4^{(*+\#)}, 34.9^{*}, 34.8^{\#}, 31.5^{(*+\#)} \mathrm{ppm}$.

LRMS (APCI) m/z: $[\mathrm{M}+\mathrm{H}]^{+}$calc'd. for $\mathrm{C}_{21} \mathrm{H}_{24} \mathrm{~F}_{3} \mathrm{NO}, 398.1$, found 398.2 .<smiles>O=C(N([18F])Cc1ccccc1)C(F)(F)Br</smiles>

\section{$N, N$-dibenzyl-2-bromo-2,2-difluoroacetamide (S49):}

An oven-dried reaction vial was cooled under $\mathrm{N}_{2}$. Dibenzylamine $(0.19 \mathrm{~mL}, 1.0 \mathrm{mmol}, 1.0$ equiv), ethyl bromodifluoroacetate $\left(0.15 \mathrm{~mL}, 1.2 \mathrm{mmol}, 1.2\right.$ equiv), and $\mathrm{La}(\mathrm{OTf})_{3}(59 \mathrm{mg}, 0.1 \mathrm{mmol}, 10$ mol \%) were added sequentially. The reaction was stirred for 20 hours then quenched with $1 \mathrm{M}$ $\mathrm{HCl}$ (aq), extracted with EtOAc (3x), filtered through a plug of silica with EtOAc, and concentrated under reduced pressure. The crude residue was purified on silica gel (20\% EtOAc/hexanes) to afford the title compound as a white amorphous solid (104 $\mathrm{mg}, 29 \%)$.

${ }^{1} \mathbf{H}$ NMR $\left(400 \mathrm{MHz}, \mathrm{CDCl}_{3}\right) \delta 7.43-7.29(\mathrm{~m}, 6 \mathrm{H}), 7.19$ (ddd, J = 7.4, 5.5, $\left.1.6 \mathrm{~Hz}, 4 \mathrm{H}\right), 4.63(\mathrm{~s}$, $2 \mathrm{H}), 4.55$ (s, 2H) ppm.

${ }^{13} \mathbf{C}$ NMR $\left(151 \mathrm{MHz}, \mathrm{CDCl}_{3}\right) \delta 160.1\left(\mathrm{t},{ }^{2} \mathrm{~J}_{\mathrm{C}-\mathrm{F}}=26.3 \mathrm{~Hz}\right), 135.5,134.8,129.2,129.1,128.4,128.4$, $128.2,127.5,111.3\left(\mathrm{t},{ }^{1} \mathrm{~J}_{\mathrm{C}-\mathrm{F}}=315.1 \mathrm{~Hz}\right), 50.6\left(\mathrm{t},{ }^{4} \mathrm{~J}_{\mathrm{C}-\mathrm{F}}=3.8 \mathrm{~Hz}\right), 48.8 \mathrm{ppm}$.

LRMS (EI) $\mathrm{m} / \mathrm{z}$ : $[\mathrm{M}]^{+}$calc'd. for $\mathrm{C}_{16} \mathrm{H}_{14} \mathrm{BrF}_{2} \mathrm{NO}, 353.0$, found 353.1.<smiles>O=C(N(Cc1ccccc1)Cc1ccccc1)C(Cl)(Cl)Cl</smiles>

\section{$N, N$-dibenzyl-2,2,2-trichloroacetamide (S50):}

Prepared according to General Acylation Procedure. Dibenzylamine $(0.66 \mathrm{~mL}, 3.3 \mathrm{mmol}, 1.1$ equiv), $\mathrm{Et}_{3} \mathrm{~N}(0.46 \mathrm{~mL}, 3.3 \mathrm{mmol}, 1.1$ equiv), and trichloroacetyl chloride $(0.33 \mathrm{~mL}, 3.0 \mathrm{mmol}, 1.0$ equiv) were stirred in $\mathrm{CH}_{2} \mathrm{Cl}_{2}(30 \mathrm{~mL}, 0.1 \mathrm{M})$ for 23 hours. Purification on silica gel $(0-10 \%$ EtOAc/hexanes) afforded the title compound as a white amorphous solid $(654 \mathrm{mg}, 64 \%)$.

${ }^{1} \mathbf{H}$ NMR $\left(400 \mathrm{MHz}, \mathrm{CDCl}_{3}\right) \delta 7.43-7.30(\mathrm{~m}, 6 \mathrm{H}), 7.24(\mathrm{~d}, \mathrm{~J}=6.9 \mathrm{~Hz}, 2 \mathrm{H}), 7.16(\mathrm{~d}, \mathrm{~J}=6.9 \mathrm{~Hz}$, $2 \mathrm{H}), 4.91(\mathrm{~s}, 2 \mathrm{H}), 4.58(\mathrm{~s}, 2 \mathrm{H}) \mathrm{ppm}$.

${ }^{1} \mathrm{H}$ NMR spectrum is consistent with reported values. ${ }^{25}$

\footnotetext{
${ }^{25}$ Diaba, F.; Montiel, J.A.; Martínez-Laporta, A.; Bonjoch, J. Dearomative radical spirocyclization from Nbenzyltrichloroacetamides revisited using a copper(I)-mediated atom transfer reaction leading to 2azaspiro[4.5]decanes. Tetrahedron Lett. 2013, 54, 2619-2622.
} 
VIII. Preparation of Spirolactam Products<smiles>CC(C)(C)N1CC2(C=CCc3[nH]ccc32)CC1=O</smiles>

1'-(tert-butyl)-1,7-dihydrospiro[indole-4,3' -pyrrolidin]-5' -one (1):

Prepared according to General Dearomative Spirolactamization Procedure 1 using $\mathrm{N}$-( ( $1 \mathrm{H}$-indol4-yl)methyl)- $N$-(tert-butyl)-2-chloroacetamide (S2) (139 mg, $0.5 \mathrm{mmol}, 1.0$ equiv), 3DPAFIPN (16.2 mg, $0.025 \mathrm{mmol}, 5 \mathrm{~mol} \%$ ), DIPEA (0.26 mL, $1.5 \mathrm{mmol}, 3.0$ equiv), and $10 \% \mathrm{H}_{2} \mathrm{O} / \mathrm{MeCN}$ $(10 \mathrm{~mL}, 0.05 \mathrm{M})$. Purification on silica gel $\left(0-10 \%\right.$ acetone $\left./ \mathrm{CH}_{2} \mathrm{Cl}_{2}\right)$ afforded the title compound as a yellow oil $(96.3 \mathrm{mg}, 79 \%)$.

Rf: 0.21 (5\% acetone/DCM)

${ }^{1} \mathbf{H}$ NMR $\left(400 \mathrm{MHz}, \mathrm{CDCl}_{3}\right) \delta 7.91(\mathrm{~s}, 1 \mathrm{H}), 6.72(\mathrm{dd}, \mathrm{J}=3.0,2.4 \mathrm{~Hz}, 1 \mathrm{H}), 6.16(\mathrm{t}, \mathrm{J}=2.7 \mathrm{~Hz}, 1 \mathrm{H})$, $5.86(\mathrm{dt}, \mathrm{J}=10.0,3.0 \mathrm{~Hz}, 1 \mathrm{H}), 5.81(\mathrm{dt}, \mathrm{J}=10.0,1.8 \mathrm{~Hz}, 1 \mathrm{H}), 3.59(\mathrm{~d}, \mathrm{~J}=9.6 \mathrm{~Hz}, 1 \mathrm{H}), 3.44(\mathrm{~d}, \mathrm{~J}$ $=9.7 \mathrm{~Hz}, 1 \mathrm{H}), 3.28-3.22(\mathrm{~m}, 2 \mathrm{H}), 2.64(\mathrm{~d}, \mathrm{~J}=16.4 \mathrm{~Hz}, 1 \mathrm{H}), 2.48(\mathrm{~d}, \mathrm{~J}=16.5 \mathrm{~Hz}, 1 \mathrm{H}), 1.43(\mathrm{~s}$, 9H) ppm.

${ }^{13} \mathrm{C}$ NMR $\left(151 \mathrm{MHz}, \mathrm{CDCl}_{3}\right) \delta 174.1,132.4,124.1,122.6,120.9,117.3,104.4,59.9,54.2,48.7$, 37.3, 28.0, $24.1 \mathrm{ppm}$.

HRMS (APCI) m/z: $[\mathrm{M}+\mathrm{H}]^{+}$calc'd. for $\mathrm{C}_{15} \mathrm{H}_{21} \mathrm{~N}_{2} \mathrm{O}, 245.16484$, found 245.16491 .

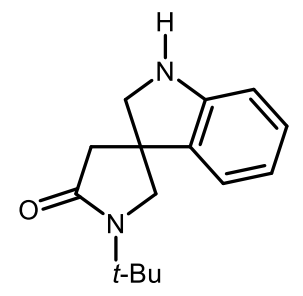

1'-(tert-butyl)spiro[indoline-3,3'-pyrrolidin]-5' -one (2):

Prepared according to General Dearomative Spirolactamization Procedure 1 using $\mathrm{N}$-( ( $1 \mathrm{H}$-indol3-yl)methyl)- $N$-(tert-butyl)-2-chloroacetamide (S4) $(56 \mathrm{mg}, 0.2 \mathrm{mmol}, 1.0$ equiv), 3DPAFIPN (6.5 mg, $0.01 \mathrm{mmol}, 5 \mathrm{~mol} \%)$, DIPEA $(0.10 \mathrm{~mL}, 0.6 \mathrm{mmol}, 3.0$ equiv), cyclohexanethiol $(1.2 \mu \mathrm{L}$, $0.01 \mathrm{mmol}, 5 \mathrm{~mol} \%$ ), and $50 \% \mathrm{H}_{2} \mathrm{O} / \mathrm{MeCN}(4 \mathrm{~mL}, 0.05 \mathrm{M}$ ). Purification on silica gel (10-50\% EtOAc/hexanes) afforded the title compound as a yellow oil (34.4 mg, 70\%).

Rf: 0.15 (30\% EtOAc/hexanes)

${ }^{1} \mathbf{H}$ NMR $\left(600 \mathrm{MHz}, \mathrm{CDCl}_{3}\right) \delta 7.15(\mathrm{~d}, \mathrm{~J}=7.4 \mathrm{~Hz}, 1 \mathrm{H}), 7.14-7.08(\mathrm{~m}, 1 \mathrm{H}), 6.83-6.77(\mathrm{~m}, 1 \mathrm{H})$, $6.71(\mathrm{~d}, \mathrm{~J}=7.8 \mathrm{~Hz}, 1 \mathrm{H}), 3.63(\mathrm{~d}, \mathrm{~J}=9.8 \mathrm{~Hz}, 1 \mathrm{H}), 3.58(\mathrm{~d}, \mathrm{~J}=9.1 \mathrm{~Hz}, 1 \mathrm{H}), 3.51(\mathrm{~d}, \mathrm{~J}=9.1 \mathrm{~Hz}$, $1 \mathrm{H}), 3.49(\mathrm{~d}, \mathrm{~J}=9.8 \mathrm{~Hz}, 1 \mathrm{H}), 2.80(\mathrm{~d}, \mathrm{~J}=16.7 \mathrm{~Hz}, 1 \mathrm{H}), 2.52(\mathrm{~d}, \mathrm{~J}=16.7 \mathrm{~Hz}, 1 \mathrm{H}), 1.42(\mathrm{~s}, 9 \mathrm{H})$ ppm.

${ }^{13} \mathrm{C}$ NMR $\left(151 \mathrm{MHz}, \mathrm{CDCl}_{3}\right) \delta 173.6,150.4,133.3,128.8,122.4,119.9,110.5,60.2,57.7,54.4$, 45.6, 45.5, $28.0 \mathrm{ppm}$.

HRMS (APCI) m/z: [M+H] $]^{+}$calc'd. for $\mathrm{C}_{15} \mathrm{H}_{21} \mathrm{~N}_{2} \mathrm{O}, 245.16484$, found 245.16487. 


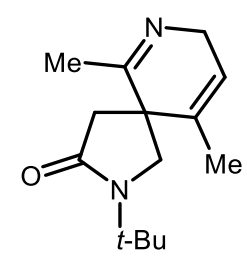

2-(tert-butyl)-6,10-dimethyl-2,7-diazaspiro[4.5]deca-6,9-dien-3-one (3):

Prepared according to General Dearomative Spirolactamization Procedure 1 using N-(tert-butyl)2-chloro-N-((2,4-dimethylpyridin-3-yl)methyl)acetamide (S8) (26.9 mg, $0.1 \mathrm{mmol}, 1.0 \mathrm{equiv})$, 3DPAFIPN (3.2 mg, $0.005 \mathrm{mmol}, 5 \mathrm{~mol} \%$ ), DIPEA (0.05 mL, $0.3 \mathrm{mmol}, 3.0$ equiv), and $50 \%$ $\mathrm{H}_{2} \mathrm{O} / \mathrm{MeCN}$ ( $2 \mathrm{~mL}, 0.05 \mathrm{M}$ ). Purification on silica gel (50-70\% acetone/DCM) afforded the title compound as a white amorphous solid (23 mg, 97\%).

Rf: 0.24 (50\% acetone/DCM)

${ }^{1} \mathbf{H}$ NMR $(500 \mathrm{MHz}, \mathrm{CDCl} 3) \delta 5.55(\mathrm{dq}, \mathrm{J}=3.1,1.5 \mathrm{~Hz}, 1 \mathrm{H}), 4.16-4.08(\mathrm{~m}, 2 \mathrm{H}), 3.49(\mathrm{~d}, \mathrm{~J}=$ $10.9 \mathrm{~Hz}, 1 \mathrm{H}), 3.42(\mathrm{~d}, \mathrm{~J}=10.9 \mathrm{~Hz}, 1 \mathrm{H}), 2.55(\mathrm{~d}, \mathrm{~J}=9.6 \mathrm{~Hz}, 2 \mathrm{H}), 2.06(\mathrm{~d}, \mathrm{~J}=1.8 \mathrm{~Hz}, 3 \mathrm{H}), 1.73(\mathrm{t}$, $\mathrm{J}=1.8 \mathrm{~Hz}, 3 \mathrm{H}), 1.42(\mathrm{~s}, 9 \mathrm{H}) \mathrm{ppm}$.

${ }^{13}$ C NMR $(101 \mathrm{MHz}, \mathrm{CDCl} 3) \delta 173.0,167.0,131.7,121.4,54.7,54.3,50.3,41.6,39.1,27.8,22.3$, $18.2 \mathrm{ppm}$.

HRMS (APCI) m/z: [M+H] ${ }^{+}$calc'd. for $\mathrm{C}_{14} \mathrm{H}_{23} \mathrm{~N}_{2} \mathrm{O}, 235.18049$, found 235.18090.

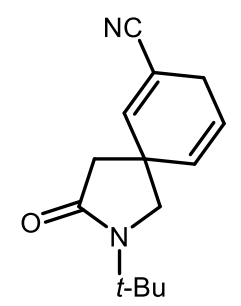

\section{2-(tert-butyl)-3-oxo-2-azaspiro[4.5]deca-6,9-diene-7-carbonitrile (4):}

Prepared according to General Dearomative Spirolactamization Procedure 2 using N-(tert-butyl)2-chloro-N-(3-cyanobenzyl)acetamide (S10) (132.4 mg, $0.5 \mathrm{mmol}, 1.0$ equiv), 3DPAFIPN (16.2 $\mathrm{mg}, 0.025 \mathrm{mmol}, 5 \mathrm{~mol} \%)$, DIPEA ( $0.26 \mathrm{~mL}, 1.5 \mathrm{mmol}, 3.0$ equiv), and $50 \% \mathrm{H}_{2} \mathrm{O} / \mathrm{MeCN}(10 \mathrm{~mL}$, $0.05 \mathrm{M})$. Purification on silica gel (0-30\% EtOAc/hexanes) afforded the title compound as a yellow amorphous solid (73 mg, 63\%).

Rf: 0.13 (20\% EtOAc/hexanes)

${ }^{1} \mathbf{H}$ NMR (600 MHz, CDCl3) $\delta 6.50$ (q, J = 2.0 Hz, 1H), 5.77 (dt, J = 10.3, 3.4 Hz, 1H), 5.67 (dq, $\mathrm{J}=10.2,2.2 \mathrm{~Hz}, 1 \mathrm{H}), 3.33(\mathrm{q}, \mathrm{J}=12 \mathrm{~Hz}, 2 \mathrm{H}), 2.85(\mathrm{dq}, \mathrm{J}=3.4,1.7 \mathrm{~Hz}, 2 \mathrm{H}), 2.35(\mathrm{~d}, \mathrm{~J}=1.6 \mathrm{~Hz}$, 2H), 1.37 (s, 9H) ppm.

${ }^{13}$ C NMR (151 MHz, CDCl3) $\delta 172.0,145.4,145.4,128.8,122.6,122.6,118.5,110.8,57.3,54.4$, 46.7, 37.6, 27.8, 278, 27.8, 27.8, $27.7 \mathrm{ppm}$.

HRMS (APCI) m/z: [M+H] ${ }^{+}$calc'd. for $\mathrm{C}_{14} \mathrm{H}_{19} \mathrm{~N}_{2} \mathrm{O}, 231.14919$, found 231.14940.

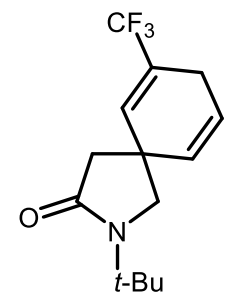

2-(tert-butyl)-7-(trifluoromethyl)-2-azaspiro[4.5]deca-6,9-dien-3-one (5): 
Prepared according to General Dearomative Spirolactamization Procedure 2 using $N$-(tert-butyl)2-chloro- $N$-(3-(trifluoromethyl)benzyl)acetamide (S12) (120 mg, 0.5 mmol, 1.0 equiv), 3DPAFIPN (16.2 mg, $0.025 \mathrm{mmol}, 5 \mathrm{~mol} \%)$, DIPEA (0.26 mL, $1.5 \mathrm{mmol}, 3.0$ equiv), and $50 \%$ $\mathrm{H}_{2} \mathrm{O} / \mathrm{MeCN}$ (10 mL, $0.05 \mathrm{M}$ ). Purification on silica gel (10-20\% EtOAc/hexanes) afforded the title compound as a yellow amorphous solid $(75 \mathrm{mg}, 55 \%)$.

Rf: 0.27 (20\% EtOAc/hexanes)

${ }^{z 1} \mathbf{H}$ NMR $\left(400 \mathrm{MHz}, \mathrm{CDCl}_{3}\right) \delta 6.26(\mathrm{p}, \mathrm{J}=1.7 \mathrm{~Hz}, 1 \mathrm{H}), 5.83(\mathrm{dt}, \mathrm{J}=10.1,3.4 \mathrm{~Hz}, 1 \mathrm{H}), 5.70(\mathrm{dq}$, $\mathrm{J}=10.0,2.1 \mathrm{~Hz}, 1 \mathrm{H}), 3.36(\mathrm{~d}, \mathrm{~J}=10.1 \mathrm{~Hz}, 1 \mathrm{H}), 3.33(\mathrm{~d}, \mathrm{~J}=10.3 \mathrm{~Hz}, 1 \mathrm{H}), 2.84-2.77(\mathrm{~m}, 2 \mathrm{H})$, $2.38(\mathrm{~s}, 2 \mathrm{H}), 1.40(\mathrm{~s}, 9 \mathrm{H}) \mathrm{ppm}$.

${ }^{13} \mathrm{C}$ NMR $\left(151 \mathrm{MHz}, \mathrm{CDCl}_{3}\right) \delta 72.7,132.1\left(\mathrm{q},{ }^{3} \mathrm{~J}_{\mathrm{C}-\mathrm{F}}=5.6 \mathrm{~Hz}\right), 129.2,126.6\left(\mathrm{q},{ }^{2} \mathrm{~J}_{\mathrm{C}-\mathrm{F}}=31.2 \mathrm{~Hz}\right)$, $123.5\left(\mathrm{q},{ }^{1} \mathrm{~J}_{\mathrm{C}-\mathrm{F}}=272.1 \mathrm{~Hz}\right), 122.8,57.7,54.4,47.0,37.0,27.9,23.7 \mathrm{ppm}$.

HRMS (APCI) m/z: [M+H] $]^{+}$calc'd. for $\mathrm{C}_{14} \mathrm{H}_{19} \mathrm{~F}_{3} \mathrm{NO}, 274.14133$, found 274.14162.<smiles>CC(C)(C)N1CC2(C=C(C(F)(F)F)CC(C(F)(F)F)=C2)CC1=O</smiles>

2-(tert-butyl)-7,9-bis(trifluoromethyl)-2-azaspiro[4.5]deca-6,9-dien-3-one (6):

Prepared according to General Dearomative Spirolactamization Procedure 1 using $N-(3,5-$ bis(trifluoromethyl)benzyl)- $N$-(tert-butyl)-2-chloroacetamide (S14) (174 mg, $0.46 \mathrm{mmol}, 1.0$ equiv), 3DPAFIPN (14.9 mg, $0.023 \mathrm{mmol}, 5 \mathrm{~mol} \%)$, DIPEA (0.24 mL, $1.38 \mathrm{mmol}, 3.0$ equiv), and $50 \% \mathrm{H}_{2} \mathrm{O} / \mathrm{MeCN}(9 \mathrm{~mL}, 0.05 \mathrm{M}$ ). Purification on silica gel (10\% EtOAc/hexanes) afforded the title compound as a yellow amorphous solid (103 $\mathrm{mg}, 66 \%)$.

Rf: 0.46 (20\% EtOAc/hexanes)

${ }^{1} \mathbf{H}$ NMR $\left(400 \mathrm{MHz}, \mathrm{CDCl}_{3}\right) \delta 6.30(\mathrm{p}, \mathrm{J}=1.8 \mathrm{~Hz}, 2 \mathrm{H}), 3.41(\mathrm{~s}, 2 \mathrm{H}), 2.97(\mathrm{~s}, 2 \mathrm{H}), 2.45(\mathrm{~s}, 2 \mathrm{H})$, $1.41(\mathrm{~s}, 9 \mathrm{H}) \mathrm{ppm}$.

${ }^{13} \mathbf{C ~ N M R}\left(151 \mathrm{MHz}, \mathrm{CDCl}_{3}\right) \delta 171.6,131.2\left(\mathrm{q},{ }^{3} \mathrm{~J}_{\mathrm{C}-\mathrm{F}}=5.4 \mathrm{~Hz}\right), 125.4\left(\mathrm{q},{ }^{2} \mathrm{~J}_{\mathrm{C}-\mathrm{F}}=31.8 \mathrm{~Hz}\right), 123.0$ $\left(\mathrm{q},{ }^{1} \mathrm{~J}_{\mathrm{C}-\mathrm{F}}=272.4 \mathrm{~Hz}\right), 56.7,54.7,46.1,37.8,27.8,21.4 \mathrm{ppm}$.

HRMS (APCI) m/z: [M+H] $]^{+}$calc'd. for $\mathrm{C}_{15} \mathrm{H}_{18} \mathrm{~F}_{6} \mathrm{NO}, 342.12871$, found 342.12899.

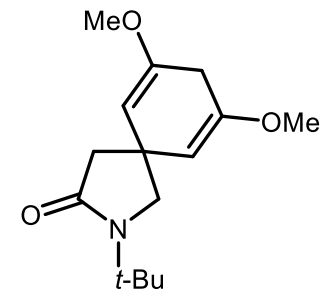

2-(tert-butyl)-7,9-dimethoxy-2-azaspiro[4.5]deca-6,9-dien-3-one (7):

Prepared according to General Dearomative Spirolactamization Procedure 1 using $N$-(3,5dimethoxybenzyl)-2-methylpropan-2-amine (S16) (60 mg, $0.2 \mathrm{mmol}, 1.0$ equiv), 3DPAFIPN (6.5 $\mathrm{mg}, 0.01 \mathrm{mmol}, 5 \mathrm{~mol} \%)$, DIPEA $\left(0.10 \mathrm{~mL}, 0.6 \mathrm{mmol}, 3.0\right.$ equiv), and $50 \% \mathrm{H}_{2} \mathrm{O} / \mathrm{MeCN}(4 \mathrm{~mL}$, $0.05 \mathrm{M})$. Purification by preparatory TLC $(20 \%$ acetone/hexanes eluent) afforded the title compound as an off-white amorphous solid (28 $\mathrm{mg}, 54 \%)$.

Rf: 0.29 (20\% acetone/hexanes) 
${ }^{1} \mathbf{H}$ NMR $\left(600 \mathrm{MHz}, \mathrm{C}_{6} \mathrm{D}_{6}\right) \delta 4.45(\mathrm{t}, \mathrm{J}=1.3 \mathrm{~Hz}, 2 \mathrm{H}), 3.13(\mathrm{~s}, 6 \mathrm{H}), 3.03(\mathrm{~s}, 2 \mathrm{H}), 2.95(\mathrm{qt}, \mathrm{J}=8.0$, $1.3 \mathrm{~Hz}, 2 \mathrm{H}), 2.35$ (s, 2H), 1.40 (s, 9H) ppm.

${ }^{13}$ C NMR (151z MHz, $\left.\mathrm{C}_{6} \mathrm{D}_{6}\right) \delta 173.3,152.9,98.4,60.1,54.0,53.6,49.5,39.4,32.0,28.0 \mathrm{ppm}$.

HRMS (APCI) m/z: [M+H] $]^{+}$calc'd. for $\mathrm{C}_{15} \mathrm{H}_{24} \mathrm{NO}_{3}, 266.17507$, found 266.17541 .

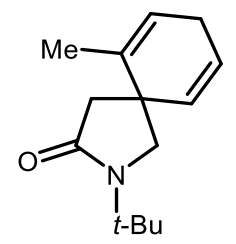

2-(tert-butyl)-6-methyl-2-azaspiro[4.5]deca-6,9-dien-3-one (8):

Prepared according to General Dearomative Spirolactamization Procedure 1 using $N$-(tert-butyl)2-chloro- $N$-(2-methylbenzyl)acetamide (S18) (126.6 mg, $0.5 \mathrm{mmol}, 1.0$ equiv), 3DPAFIPN (16.2 $\mathrm{mg}, 0.025 \mathrm{mmol}, 5 \mathrm{~mol} \%)$, DIPEA $\left(0.26 \mathrm{~mL}, 1.5 \mathrm{mmol}, 3.0\right.$ equiv), and $50 \% \mathrm{H}_{2} \mathrm{O} / \mathrm{MeCN}(10 \mathrm{~mL}$, $0.05 \mathrm{M})$. Purification on silica gel $(5-10 \%$ acetone/hexanes) afforded the title compound as a yellow oil (72 mg, 66\%).

Rf: 0.27 (10\% acetone/hexanes)

${ }^{1}$ H NMR (600 MHz, CDCl3) $\delta 5.70(\mathrm{dtd}, \mathrm{J}=9.8,3.3,1.5 \mathrm{~Hz}, 1 \mathrm{H}), 5.57(\mathrm{dt}, \mathrm{J}=9.9,2.1 \mathrm{~Hz}, 1 \mathrm{H})$, $5.46(\mathrm{tt}, \mathrm{J}=3.4,1.6 \mathrm{~Hz}, 1 \mathrm{H}), 3.45(\mathrm{~d}, \mathrm{~J}=10.2 \mathrm{~Hz}, 1 \mathrm{H}), 3.21(\mathrm{~d}, \mathrm{~J}=10.2 \mathrm{~Hz}, 1 \mathrm{H}), 2.59(\mathrm{tq}, \mathrm{J}=3.7$, $1.9 \mathrm{~Hz}, 2 \mathrm{H}), 2.49(\mathrm{~d}, \mathrm{~J}=17.1 \mathrm{~Hz}, 1 \mathrm{H}), 2.23(\mathrm{~d}, \mathrm{~J}=17.1 \mathrm{~Hz}, 1 \mathrm{H}), 1.72(\mathrm{q}, \mathrm{J}=1.8 \mathrm{~Hz}, 3 \mathrm{H}), 1.37$ (s, 9H) $\mathrm{ppm}$.

${ }^{13}$ C NMR (151 MHz, CDCl3) $\delta 173.7,134.7,131.9,123.6,121.6,57.4,54.2,45.8,37.9,27.8$, 27.0, $19.0 \mathrm{ppm}$.

HRMS (APCI) m/z: [M+H] ${ }^{+}$calc'd. for $\mathrm{C}_{14} \mathrm{H}_{22} \mathrm{NO}, 220.16959$, found 220.16959.

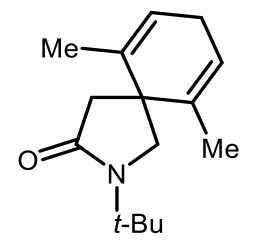

2-(tert-butyl)-6,10-dimethyl-2-azaspiro[4.5]deca-6,9-dien-3-one (9):

Prepared according to General Dearomative Spirolactamization Procedure 1 using $N$-(tert-butyl)2-chloro- $N$-(2,6-dimethylbenzyl)acetamide (S20) (133.9 mg, $0.5 \mathrm{mmol}, 1.0$ equiv), 3DPAFIPN (16.2 mg, $0.025 \mathrm{mmol}, 5 \mathrm{~mol} \%$ ), DIPEA (0.26 mL, $1.5 \mathrm{mmol}, 3.0$ equiv), and $50 \% \mathrm{H}_{2} \mathrm{O} / \mathrm{MeCN}$ $(10 \mathrm{~mL}, 0.05 \mathrm{M})$. Purification on silica gel (10-15\% acetone/hexanes) afforded the title compound as a yellow oil (98 $\mathrm{mg}, 84 \%)$.

Rf: 0.24 (10\% acetone/hexanes)

${ }^{1} \mathbf{H}$ NMR (600 MHz, CDCl3) $\delta 5.47(\mathrm{t}, \mathrm{J}=3.5 \mathrm{~Hz}, 2 \mathrm{H}), 3.39(\mathrm{~s}, 2 \mathrm{H}), 2.59(\mathrm{tt}, \mathrm{J}=3.7,1.9 \mathrm{~Hz}, 2 \mathrm{H})$, $2.47(\mathrm{~s}, 2 \mathrm{H}), 1.75-1.72(\mathrm{~m}, 6 \mathrm{H}), 1.41(\mathrm{~s}, 9 \mathrm{H}) \mathrm{ppm}$.

${ }^{13}$ C NMR (151 MHz, CDCl3) $\delta 174.1,134.8,121.3,55.6,54.5,42.5,39.8,27.8,27.3,18.9$ ppm. HRMS (APCI) m/z: [M+H] $]^{+}$calc'd. for $\mathrm{C}_{15} \mathrm{H}_{24} \mathrm{NO}, 234.18524$, found 234.18562 . 


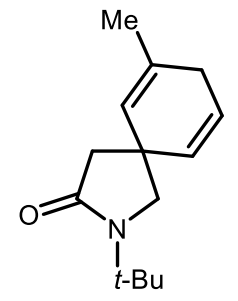

2-(tert-butyl)-7-methyl-2-azaspiro[4.5]deca-6,9-dien-3-one (10):

Prepared according to General Dearomative Spirolactamization Procedure 1 using $N$-(tert-butyl)2-chloro-N-(3-methylbenzyl)acetamide (S22) (126.9 mg, $0.5 \mathrm{mmol}, 1.0$ equiv), 3DPAFIPN (16.2 $\mathrm{mg}, 0.025 \mathrm{mmol}, 5 \mathrm{~mol} \%)$, DIPEA ( $0.26 \mathrm{~mL}, 1.5 \mathrm{mmol}, 3.0$ equiv), and $50 \% \mathrm{H}_{2} \mathrm{O} / \mathrm{MeCN}$ (10 mL, $0.05 \mathrm{M})$. Purification on silica gel (10-20\% EtOAc/hexanes) afforded the title compound as a yellow oil (65 mg, $59 \%)$.

Rf: 0.34 (20\% EtOAc/hexanes)

${ }^{1} \mathbf{H}$ NMR $(600 \mathrm{MHz}, \mathrm{CDCl} 3) \delta 5.74(\mathrm{dt}, \mathrm{J}=10.0,3.3 \mathrm{~Hz}, 1 \mathrm{H}), 5.65(\mathrm{dq}, \mathrm{J}=10.0,2.1 \mathrm{~Hz}, 1 \mathrm{H})$, $5.35(\mathrm{~s}, 1 \mathrm{H}), 3.24(\mathrm{~s}, 2 \mathrm{H}), 2.54-2.50(\mathrm{~m}, 2 \mathrm{H}), 2.26(\mathrm{~s}, 2 \mathrm{H}), 1.69(\mathrm{~s}, 3 \mathrm{H}), 1.37(\mathrm{~s}, 9 \mathrm{H}) \mathrm{ppm}$.

${ }^{13} \mathrm{C}$ NMR $(151 \mathrm{MHz}, \mathrm{CDCl} 3) \delta 173.8,132.1,130.2,125.0,124.3,58.7,54.0,47.9,37.6,31.2$, 27.9, $23.2 \mathrm{ppm}$.

HRMS (APCI) m/z: [M+H] ${ }^{+}$calc'd. for $\mathrm{C}_{14} \mathrm{H}_{22} \mathrm{NO}, 220.16959$, found 220.16966.<smiles>CC1C(=O)N(C(C)(C)C)CC12C=CCC=C2</smiles>

2-(tert-butyl)-4-methyl-2-azaspiro[4.5]deca-6,9-dien-3-one (11):

Prepared according to General Dearomative Spirolactamization Procedure 1 using $N$-benzyl- $N$ (tert-butyl)-2-chloropropanamide (S24) (127 mg, $0.5 \mathrm{mmol}, 1.0$ equiv), 3DPAFIPN (16.2 mg, $0.025 \mathrm{mmol}, 5 \mathrm{~mol} \%)$, DIPEA (0.26 mL, $1.5 \mathrm{mmol}, 3.0$ equiv), and 50\% $\mathrm{H}_{2} \mathrm{O} / \mathrm{MeCN}(10 \mathrm{~mL}, 0.05$ M). Purification on silica gel (2-3\% acetone/hexanes) afforded the title compound as an off-white amorphous solid (94 mg, 85\%).

Rf: 0.26 (5\% acetone/hexanes)

${ }^{1} \mathbf{H}$ NMR $\left(400 \mathrm{MHz}, \mathrm{CDCl}_{3}\right) \delta 5.93-5.81(\mathrm{~m}, 2 \mathrm{H}), 5.55(\mathrm{dq}, \mathrm{J}=9.6,1.9 \mathrm{~Hz}, 1 \mathrm{H}), 5.48(\mathrm{dq}, \mathrm{J}=$ 10.0, 2.1 Hz, 1H), 3.24 (d, J = 9.7 Hz, 1H), 3.19 (d, J = 9.8 Hz, 1H), $2.70-2.62(\mathrm{~m}, 1 \mathrm{H}), 2.24$ (q, $\mathrm{J}=7.3 \mathrm{~Hz}, 1 \mathrm{H}), 1.38(\mathrm{~s}, 9 \mathrm{H}), 0.94(\mathrm{~d}, \mathrm{~J}=7.3 \mathrm{~Hz}, 3 \mathrm{H}) \mathrm{ppm}$.

${ }^{13} \mathrm{C}$ NMR $\left(151 \mathrm{MHz}, \mathrm{CDCl}_{3}\right) \delta 175.9,130.0,127.2,127.0,125.8,56.6,53.9,49.3,41.9,27.9,26.8$, $9.7 \mathrm{ppm}$.

HRMS (APCI) m/z: [M+H] ${ }^{+}$calc'd. for $\mathrm{C}_{14} \mathrm{H}_{22} \mathrm{NO}, 220.16959$, found 220.16969 .

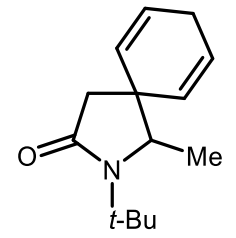

2-(tert-butyl)-1-methyl-2-azaspiro[4.5]deca-6,9-dien-3-one (12):

Prepared according to General Dearomative Spirolactamization Procedure 2 using $N$-(tert-butyl)2-chloro- $N$-(1-phenylethyl)acetamide (S26) (254 mg, $1.0 \mathrm{mmol}, 1.0$ equiv), 3DPAFIPN (32.4 mg, 
$0.05 \mathrm{mmol}, 5 \mathrm{~mol} \%)$, DIPEA (0.52 mL, $3.0 \mathrm{mmol}, 3.0$ equiv), and 50\% $\mathrm{H}_{2} \mathrm{O} / \mathrm{MeCN}(20 \mathrm{~mL}, 0.05$ M). Purification on silica gel (10-20\% EtOAc/hexanes) afforded the title compound as a yellow amorphous solid (89.7 mg, 41\%).

Rf: 0.45 (20\% EtOAc/hexanes)

${ }^{1} \mathbf{H}$ NMR $\left(400 \mathrm{MHz}, \mathrm{CDCl}_{3}\right) \delta 5.88(\mathrm{dtd}, \mathrm{J}=10.3,3.4,1.7 \mathrm{~Hz}, 1 \mathrm{H}), 5.81(\mathrm{dq}, \mathrm{J}=10.2,2.0 \mathrm{~Hz}$, $1 \mathrm{H}), 5.72(\mathrm{dtd}, \mathrm{J}=10.2,3.3,1.7 \mathrm{~Hz}, 1 \mathrm{H}), 5.60(\mathrm{dq}, \mathrm{J}=10.3,2.1 \mathrm{~Hz}, 1 \mathrm{H}), 3.49(\mathrm{q}, \mathrm{J}=6.5 \mathrm{~Hz}, 1 \mathrm{H})$, $2.64(\mathrm{~m}, 2 \mathrm{H}), 2.51(\mathrm{~d}, \mathrm{~J}=16.6 \mathrm{~Hz}, 1 \mathrm{H}), 2.11(\mathrm{~d}, \mathrm{~J}=16.6 \mathrm{~Hz}, 1 \mathrm{H}), 1.43(\mathrm{~s}, 9 \mathrm{H}), 1.20(\mathrm{~d}, \mathrm{~J}=6.4$ $\mathrm{Hz}, 3 \mathrm{H}) \mathrm{ppm}$.

${ }^{13}$ C NMR $\left(151 \mathrm{MHz}, \mathrm{CDCl}_{3}\right) \delta$ 73.0, 131.3, 127.9, 127.0, 123.3, 64.9, 54.2, 45.5, 40.2, 28.4, 26.7, $17.7 \mathrm{ppm}$.

HRMS (APCI) m/z: [M+H] ${ }^{+}$calc'd. for $\mathrm{C}_{14} \mathrm{H}_{22} \mathrm{NO}, 220.16959$, found 220.16983.<smiles>CC(C)(C)N1CC2(C=CCC=C2F)CC1=O</smiles>

2-(tert-butyl)-6-fluoro-2-azaspiro[4.5] deca-6,9-dien-3-one (13):

Prepared according to General Dearomative Spirolactamization Procedure 1 using $N$-(tert-butyl)2-chloro- $N$-(2-fluorobenzyl)acetamide (S28) (128.6 mg, $0.5 \mathrm{mmol}, 1.0$ equiv), 3DPAFIPN (16.2 $\mathrm{mg}, 0.025 \mathrm{mmol}, 5 \mathrm{~mol} \%$ ), DIPEA ( $0.26 \mathrm{~mL}, 1.5 \mathrm{mmol}, 3.0$ equiv), and $50 \% \mathrm{H}_{2} \mathrm{O} / \mathrm{MeCN}$ (10 mL, $0.05 \mathrm{M})$. Purification on silica gel (0-20\% EtOAc/hexanes) afforded the title compound as a yellow amorphous solid (81 $\mathrm{mg}, 72 \%)$.

Rf: 0.32 (20\% EtOAc/hexanes)

${ }^{1}$ H NMR $(600 \mathrm{MHz}, \mathrm{CDCl} 3) \delta 5.72-5.64(\mathrm{~m}, 2 \mathrm{H}), 5.33(\mathrm{dt}, \mathrm{J}=17.5,3.9 \mathrm{~Hz}, 1 \mathrm{H}), 3.71(\mathrm{~d}, \mathrm{~J}=$ $9.8 \mathrm{~Hz}, 1 \mathrm{H}), 3.27(\mathrm{~d}, \mathrm{~J}=9.9 \mathrm{~Hz}, 1 \mathrm{H}), 2.83-2.77(\mathrm{~m}, 3 \mathrm{H}), 2.25(\mathrm{~d}, \mathrm{~J}=16.7 \mathrm{~Hz}, 1 \mathrm{H}), 1.40(\mathrm{~d}, \mathrm{~J}=$ $0.8 \mathrm{~Hz}, 9 \mathrm{H}) \mathrm{ppm}$.

${ }^{13} \mathbf{C ~ N M R}(151 \mathrm{MHz}, \mathrm{CDCl} 3) \delta 172.6,158.4\left(\mathrm{~d},{ }^{1} \mathrm{~J}_{\mathrm{C}-\mathrm{F}}=254.7 \mathrm{~Hz}\right), 130.2\left(\mathrm{~d},{ }^{3} \mathrm{~J}_{\mathrm{C}-\mathrm{F}}=6.4 \mathrm{~Hz}\right), 123.2$ $\left(\mathrm{d},{ }^{4} \mathrm{~J}_{\mathrm{C}-\mathrm{F}}=2.7 \mathrm{~Hz}\right), 101.7\left(\mathrm{~d},{ }^{2} \mathrm{~J}_{\mathrm{C}-\mathrm{F}}=17.2 \mathrm{~Hz}\right), 55.4,54.4,43.6,37.8\left(\mathrm{~d},{ }^{2} \mathrm{~J}_{\mathrm{C}-\mathrm{F}}=23.2 \mathrm{~Hz}\right), 27.8,26.4$ $\left(\mathrm{d},{ }^{3} \mathrm{~J}_{\mathrm{C}-\mathrm{F}}=7.0 \mathrm{~Hz}\right) \mathrm{ppm}$.

HRMS (APCI) m/z: [M+H] ${ }^{+}$calc'd. for $\mathrm{C}_{13} \mathrm{H}_{19} \mathrm{FNO}, 224.14452$, found 224.14472.<smiles>CC(C)(C)N1CC2(C=CCC(F)=C2)CC1=O</smiles>

\section{2-(tert-butyl)-7-fluoro-2-azaspiro[4.5]deca-6,9-dien-3-one (14):}

Prepared according to General Dearomative Spirolactamization Procedure 2 using $N$-(tert-butyl)2-chloro- $N$-(3-fluorobenzyl)acetamide (S30) (129 mg, $0.5 \mathrm{mmol}, 1.0$ equiv), 3DPAFIPN (16.2 $\mathrm{mg}, 0.025 \mathrm{mmol}, 5 \mathrm{~mol} \%)$, DIPEA ( $0.26 \mathrm{~mL}, 1.5 \mathrm{mmol}, 3.0$ equiv), and $50 \% \mathrm{H}_{2} \mathrm{O} / \mathrm{MeCN}(10 \mathrm{~mL}$, $0.05 \mathrm{M})$. Purification on silica gel (10-20\% EtOAc/hexanes) afforded the title compound as a yellow amorphous solid (84.7 $\mathrm{mg}, 76 \%)$.

Rf: 0.26 (20\% EtOAc/hexanes) 
${ }^{1} \mathbf{H}$ NMR $\left(600 \mathrm{MHz}, \mathrm{CDCl}_{3}\right) \delta 5.69(\mathrm{ddt}, \mathrm{J}=9.9,7.8,3.3 \mathrm{~Hz}, 1 \mathrm{H}), 5.64(\mathrm{ddq}, \mathrm{J}=9.8,3.7,1.9 \mathrm{~Hz}$, $1 \mathrm{H}), 5.24(\mathrm{dq}, \mathrm{J}=17.0,1.5 \mathrm{~Hz}, 1 \mathrm{H}), 3.34(\mathrm{dd}, \mathrm{J}=9.9,1.1 \mathrm{~Hz}, 1 \mathrm{H}), 3.32(\mathrm{~d}, \mathrm{~J}=9.9 \mathrm{~Hz}, 1 \mathrm{H}), 2.86$ $-2.82(\mathrm{~m}, 2 \mathrm{H}), 2.36(\mathrm{~d}, \mathrm{~J}=1.0 \mathrm{~Hz}, 1 \mathrm{H}), 2.35(\mathrm{~d}, \mathrm{~J}=16.6 \mathrm{~Hz}, 1 \mathrm{H}), 1.39(\mathrm{~s}, 9 \mathrm{H}) \mathrm{ppm}$.

${ }^{13} \mathbf{C}$ NMR $\left(151 \mathrm{MHz}, \mathrm{CDCl}_{3}\right) \delta 173.2,158.5\left(\mathrm{~d},{ }^{1} \mathrm{~J}_{\mathrm{C}-\mathrm{F}}=256.0 \mathrm{~Hz}\right), 130.5\left(\mathrm{~d},{ }^{4} \mathrm{~J}_{\mathrm{C}-\mathrm{F}}=2.8 \mathrm{~Hz}\right), 121.9$ $\left(\mathrm{d},{ }^{3} \mathrm{~J}_{\mathrm{C}-\mathrm{F}}=10.6 \mathrm{~Hz}\right), 106.3\left(\mathrm{~d},{ }^{2} \mathrm{~J}_{\mathrm{C}-\mathrm{F}}=15.0 \mathrm{~Hz}\right), 58.4\left(\mathrm{~d},{ }^{4} \mathrm{~J}_{\mathrm{C}-\mathrm{F}}=2.6 \mathrm{~Hz}\right), 54.2,47.6\left(\mathrm{~d},{ }^{4} \mathrm{~J}_{\mathrm{C}-\mathrm{F}}=2.0\right.$ $\mathrm{Hz}), 39.5\left(\mathrm{~d},{ }^{3} \mathrm{~J}_{\mathrm{C}-\mathrm{F}}=7.8 \mathrm{~Hz}\right), 27.9,27.2\left(\mathrm{~d},{ }^{2} \mathrm{~J}_{\mathrm{C}-\mathrm{F}}=26.4 \mathrm{~Hz}\right) \mathrm{ppm}$.

HRMS (APCI) m/z: $[\mathrm{M}+\mathrm{H}]^{+}$calc'd. for $\mathrm{C}_{13} \mathrm{H}_{19} \mathrm{FNO}, 224.14452$, found 224.14468.

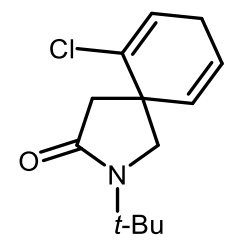

2-(tert-butyl)-6-chloro-2-azaspiro[4.5]deca-6,9-dien-3-one (15):

Prepared according to General Dearomative Spirolactamization Procedure 1 using $N$-(tert-butyl)2-chloro- $N$-(2-chlorobenzyl)acetamide (S32) (137.1 mg, $0.5 \mathrm{mmol}, 1.0$ equiv), 3DPAFIPN (16.2 $\mathrm{mg}, 0.025 \mathrm{mmol}, 5 \mathrm{~mol} \%$ ), DIPEA ( $0.26 \mathrm{~mL}, 1.5 \mathrm{mmol}, 3.0$ equiv), and $50 \% \mathrm{H}_{2} \mathrm{O} / \mathrm{MeCN}(10 \mathrm{~mL}$, $0.05 \mathrm{M})$. Purification on silica gel (5-10\% acetone/hexanes) afforded the title compound as a yellow oil (57 mg, 47\%).

Rf: 0.22 (10\% acetone/hexanes)

${ }^{1}$ H NMR (600 MHz, CDCl3) $\delta 5.93(\mathrm{t}, \mathrm{J}=3.7 \mathrm{~Hz}, 1 \mathrm{H}), 5.75-5.67(\mathrm{~m}, 2 \mathrm{H}), 3.79$ (d, J = 10.1 Hz, $1 \mathrm{H}), 3.27(\mathrm{~d}, \mathrm{~J}=10.1 \mathrm{~Hz}, 1 \mathrm{H}), 2.92(\mathrm{~d}, \mathrm{~J}=17.0 \mathrm{~Hz}, 1 \mathrm{H}), 2.79(\mathrm{dq}, \mathrm{J}=4.0,2.4,2.0 \mathrm{~Hz}, 2 \mathrm{H}), 2.27$ $(\mathrm{d}, \mathrm{J}=16.9 \mathrm{~Hz}, 1 \mathrm{H}), 1.41(\mathrm{~s}, 9 \mathrm{H}) \mathrm{ppm}$.

${ }^{13}$ C NMR (151 MHz, CDCl3) $\delta$ 172.8, 134.4, 130.9, 124.8, 122.0, 56.82, 54.5, 45.2, 40.4, 28.5, $27.8 \mathrm{ppm}$.

HRMS (APCI) m/z: $[\mathrm{M}+\mathrm{H}]^{+}$calc'd. for $\mathrm{C}_{13} \mathrm{H}_{19} \mathrm{ClNO}, 240.11497$, found 240.11541 .

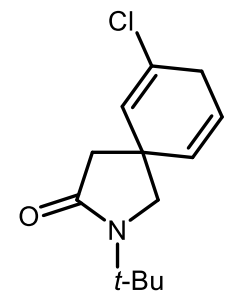

\section{2-(tert-butyl)-7-chloro-2-azaspiro[4.5]deca-6,9-dien-3-one (16):}

Prepared according to General Dearomative Spirolactamization Procedure 1 using $N$-(tert-butyl)2-chloro- $N$-(3-chlorobenzyl)acetamide (S34) (137.1 mg, $0.5 \mathrm{mmol}, 1.0$ equiv), 3DPAFIPN (16.2 $\mathrm{mg}, 0.025 \mathrm{mmol}, 5 \mathrm{~mol} \%)$, DIPEA ( $0.26 \mathrm{~mL}, 1.5 \mathrm{mmol}, 3.0$ equiv), and $50 \% \mathrm{H}_{2} \mathrm{O} / \mathrm{MeCN}(10 \mathrm{~mL}$, $0.05 \mathrm{M})$. Purification on silica gel $(10 \% \mathrm{EtOAc/hexanes)}$ afforded the title compound as a yellow amorphous solid (84 mg, 70\%).

Rf: 0.31 (20\% EtOAc/hexanes)

${ }^{1} \mathbf{H}$ NMR (600 MHz, CDCl3) $\delta 5.78(\mathrm{q}, \mathrm{J}=1.8 \mathrm{~Hz}, 1 \mathrm{H}), 5.69$ (dt, J = 10.0, $\left.3.3 \mathrm{~Hz}, 1 \mathrm{H}\right), 5.63$ (dq, $\mathrm{J}=10.0,2.0 \mathrm{~Hz}, 1 \mathrm{H}), 3.30(\mathrm{dd}, \mathrm{J}=18.0,12.0 \mathrm{~Hz}, 2 \mathrm{H}), 2.89(\mathrm{dt}, \mathrm{J}=3.4,2.0 \mathrm{~Hz}, 2 \mathrm{H}), 2.33(\mathrm{~d}, \mathrm{~J}=$ $1.9 \mathrm{~Hz}, 2 \mathrm{H}), 1.36(\mathrm{~s}, 9 \mathrm{H}) \mathrm{ppm}$.

${ }^{13}$ C NMR (151 MHz, CDCl3) $\delta$ 173.0, 131.0, 129.4, 127.2, 123.4, 57.9, 54.3, 47.1, 40.0, 33.6, $27.8 \mathrm{ppm}$.

HRMS (APCI) m/z: $[\mathrm{M}+\mathrm{H}]^{+}$calc'd. for $\mathrm{C}_{13} \mathrm{H}_{19} \mathrm{ClNO}, 240.11497$, found 240.11508 . 


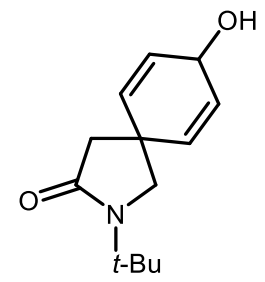

2-(tert-butyl)-8-hydroxy-2-azaspiro[4.5]deca-6,9-dien-3-one (17):

Prepared according to General Dearomative Spirolactamization Procedure 1 using $N$-(tert-butyl)2-chloro- $N$-(4-chlorobenzyl)acetamide (S36) (137 mg, $0.5 \mathrm{mmol}, 1.0$ equiv), 3DPAFIPN (16.2 $\mathrm{mg}, 0.025 \mathrm{mmol}, 5 \mathrm{~mol} \%)$, DIPEA ( $0.26 \mathrm{~mL}, 1.5 \mathrm{mmol}$, 3.0 equiv), and $50 \% \mathrm{H}_{2} \mathrm{O} / \mathrm{MeCN}(10 \mathrm{~mL}$, $0.05 \mathrm{M})$. Purification on silica gel (50-75\% EtOAc/hexanes) afforded the title compound (72.2 mg, $65 \%, 1: 1$ d.r.).

Diastereomer 1 (elutes first, $36.2 \mathrm{mg}$, yellow amorphous solid):

Rf: 0.30 (75\% EtOAc/hexanes)

${ }^{1} \mathbf{H}$ NMR (400 MHz, $\left.\mathrm{CDCl}_{3}\right) \delta 5.93(\mathrm{dq}, \mathrm{J}=10.3,3.0 \mathrm{~Hz}, 2 \mathrm{H}), 5.88(\mathrm{dd}, \mathrm{J}=10.3,1.3 \mathrm{~Hz}, 2 \mathrm{H})$, $4.54(\mathrm{~s}, 1 \mathrm{H}), 3.33(\mathrm{~s}, 2 \mathrm{H}), 2.32(\mathrm{~s}, 2 \mathrm{H}), 1.39$ (s, 9H) ppm.

${ }^{13} \mathrm{C}$ NMR $\left(101 \mathrm{MHz}, \mathrm{CDCl}_{3}\right) \delta 173.0,132.9,128.1,62.3,56.5,54.3,46.2,37.2,27.9 \mathrm{ppm}$.

HRMS (APCI) m/z: [M+H] ${ }^{+}$calc'd. for $\mathrm{C}_{13} \mathrm{H}_{20} \mathrm{NO}_{2}, 222.14886$, found 222.14877.

Diastereomer 2 (elutes second, 36.0 mg, yellow amorphous solid):

Rf: 0.22 (75\% EtOAc/hexanes)

${ }^{1} \mathbf{H}$ NMR $\left(400 \mathrm{MHz}, \mathrm{CDCl}_{3}\right) \delta 5.94(\mathrm{dq}, \mathrm{J}=10.3,3.1 \mathrm{~Hz}, 2 \mathrm{H}), 5.87$ (dq, J = 10.2, $\left.1.5 \mathrm{~Hz}, 2 \mathrm{H}\right)$, $4.51(\mathrm{~s}, 1 \mathrm{H}), 3.28(\mathrm{~s}, 2 \mathrm{H}), 2.37(\mathrm{~s}, 2 \mathrm{H}), 1.39(\mathrm{~s}, 9 \mathrm{H}) \mathrm{ppm}$.

${ }^{13} \mathrm{C} \mathrm{NMR}\left(101 \mathrm{MHz}, \mathrm{CDCl}_{3}\right) \delta 173.2,132.9,128.2,62.2,57.0,54.3,45.7,37.2,27.9 \mathrm{ppm}$.

HRMS (APCI) m/z: [M+H] ${ }^{+}$calc'd. for $\mathrm{C}_{13} \mathrm{H}_{20} \mathrm{NO}_{2}, 222.14886$, found 222.14897.

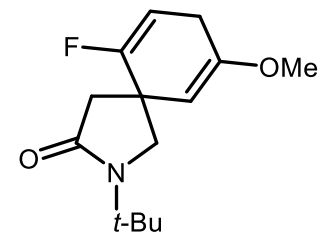

2-(tert-butyl)-6-fluoro-9-methoxy-2-azaspiro[4.5]deca-6,9-dien-3-one (18):

Prepared according to General Dearomative Spirolactamization Procedure 1 using $N$-(tert-butyl)2-chloro- $N$-(2-fluoro-5-methoxybenzyl)acetamide (S38) (143.9 mg, $0.5 \mathrm{mmol}, 1.0$ equiv), 3DPAFIPN (16.2 mg, $0.025 \mathrm{mmol}, 5 \mathrm{~mol} \%)$, DIPEA (0.26 mL, $1.5 \mathrm{mmol}, 3.0$ equiv), and 50\% $\mathrm{H}_{2} \mathrm{O} / \mathrm{MeCN}$ (10 mL, $\left.0.05 \mathrm{M}\right)$. Purification on silica gel (10-20\% EtOAc/hexanes) afforded the title compound as a yellow oil (88 $\mathrm{mg}, 69 \%)$.

Rf: 0.26 (20\% EtOAc/hexanes)

${ }^{1}$ H NMR (600 MHz, C6D6) $\delta 4.86$ (dt, J = 16.1, 3.7 Hz, 1H), $4.36(\mathrm{~d}, \mathrm{~J}=7.3 \mathrm{~Hz}, 1 \mathrm{H}), 3.52(\mathrm{dd}, \mathrm{J}$ $=9.6,1.0 \mathrm{~Hz}, 1 \mathrm{H}), 3.01(\mathrm{~s}, 3 \mathrm{H}), 2.95(\mathrm{~d}, \mathrm{~J}=9.6 \mathrm{~Hz}, 1 \mathrm{H}), 2.87(\mathrm{~d}, \mathrm{~J}=16.5 \mathrm{~Hz}, 1 \mathrm{H}), 2.61-2.48$ $(\mathrm{m}, 2 \mathrm{H}), 2.23(\mathrm{~d}, \mathrm{~J}=16.5 \mathrm{~Hz}, 1 \mathrm{H}), 1.32(\mathrm{~s}, 9 \mathrm{H}) \mathrm{ppm}$.

${ }^{13} \mathbf{C}$ NMR $(151 \mathrm{MHz}, \mathrm{C} 6 \mathrm{D} 6) \delta 172.0,159.1\left(\mathrm{~d},{ }^{1} \mathrm{~J}_{\mathrm{C}-\mathrm{F}}=254.5 \mathrm{~Hz}\right), 153.3\left(\mathrm{~d},{ }^{4} \mathrm{~J}_{\mathrm{C}-\mathrm{F}}=2.8 \mathrm{~Hz}\right), 99.8$ $\left(\mathrm{d},{ }^{2} \mathrm{~J}_{\mathrm{C}-\mathrm{F}}=20.1 \mathrm{~Hz}\right), 98.5\left(\mathrm{~d},{ }^{3} \mathrm{~J}_{\mathrm{C}-\mathrm{F}}=7.4 \mathrm{~Hz}\right), 56.39,54.2,44.7,38.8\left(\mathrm{~d},{ }^{2} \mathrm{~J}_{\mathrm{C}-\mathrm{F}}=23.8 \mathrm{~Hz}\right), 28.3(\mathrm{~d}$, $\left.{ }^{3} \mathrm{~J}_{\mathrm{C}-\mathrm{F}}=8.2 \mathrm{~Hz}\right), 27.7 \mathrm{ppm}$.

HRMS (APCI) m/z: [M+H] $]^{+}$calc'd. for $\mathrm{C}_{14} \mathrm{H}_{21} \mathrm{FNO}_{2}, 254.15508$, found 254.15549. 


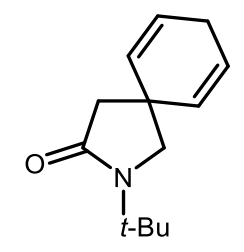

2-(tert-butyl)-2-azaspiro[4.5]deca-6,9-dien-3-one (19):

Prepared according to General Dearomative Spirolactamization Procedure 1 using $N$-benzyl- $N$ (tert-butyl)-2-chloroacetamide (S39) (120 mg, 0.5 mmol, 1.0 equiv), 3DPAFIPN (16.2 mg, 0.025 mmol, $5 \mathrm{~mol} \%)$, DIPEA (0.26 mL, $1.5 \mathrm{mmol}, 3.0$ equiv), and $50 \% \mathrm{H}_{2} \mathrm{O} / \mathrm{MeCN}(10 \mathrm{~mL}, 0.05 \mathrm{M})$. Purification on silica gel (10\% EtOAc/hexanes) afforded the title compound as a white amorphous solid (103 mg, 73\%).

Rf: 0.28 (20\% EtOAc/hexanes)

${ }^{1} \mathbf{H}$ NMR (600 MHz, CDCl3) $\delta 5.77(\mathrm{dt}, \mathrm{J}=10.1,3.3 \mathrm{~Hz}, 2 \mathrm{H}), 5.66(\mathrm{dt}, \mathrm{J}=10.3,2.1 \mathrm{~Hz}, 2 \mathrm{H}), 3.28$ $(\mathrm{s}, 2 \mathrm{H}), 2.63(\mathrm{p}, \mathrm{J}=2.7 \mathrm{~Hz}, 2 \mathrm{H}), 2.31(\mathrm{~s}, 2 \mathrm{H}), 1.38(\mathrm{~s}, 9 \mathrm{H}) \mathrm{ppm}$.

${ }^{13}$ C NMR (151 MHz, CDCl3) $\delta$ 173.7, 130.3, 124.7, 58.8, 54.1, 48.0, 36.4, 27.9, 26.4 ppm.

HRMS (APCI) m/z: [M+H] ${ }^{+}$calc'd. for $\mathrm{C}_{13} \mathrm{H}_{20} \mathrm{NO}, 206.15394$, found 206.15423.

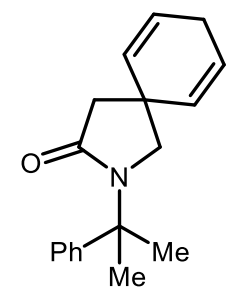

\section{2-(2-phenylpropan-2-yl)-2-azaspiro[4.5]deca-6,9-dien-3-one (S51):}

Prepared according to General Dearomative Spirolactamization Procedure 1 using $N$-benzyl-2chloro- $N$-(2-phenylpropan-2-yl)acetamide (S41) (121 mg, 0.4 mmol, 1.0 equiv), 3DPAFIPN (13.0 $\mathrm{mg}, 0.020 \mathrm{mmol}, 5 \mathrm{~mol} \%)$, DIPEA $\left(0.21 \mathrm{~mL}, 1.2 \mathrm{mmol}, 3.0\right.$ equiv), and $50 \% \mathrm{H}_{2} \mathrm{O} / \mathrm{MeCN}(8 \mathrm{~mL}$, $0.05 \mathrm{M})$. Purification on silica gel (10-20\% EtOAc/hexanes) afforded the title compound as a white amorphous solid (63 mg, 59\%).

Rf: 0.36 (20\% EtOAc/hexanes)

${ }^{1} \mathbf{H}$ NMR $(600 \mathrm{MHz}, \mathrm{CDCl} 3) \delta 7.37-7.28(\mathrm{~m}, 4 \mathrm{H}), 7.23(\mathrm{tt}, \mathrm{J}=6.6,2.1 \mathrm{~Hz}, 1 \mathrm{H}), 5.78(\mathrm{dt}, \mathrm{J}=$ 10.3, 3.3 Hz, 2H), $5.70(\mathrm{dt}, \mathrm{J}=10.3,2.1 \mathrm{~Hz}, 2 \mathrm{H}), 3.22(\mathrm{~s}, 2 \mathrm{H}), 2.63(\mathrm{dh}, \mathrm{J}=7.4,2.2 \mathrm{~Hz}, 2 \mathrm{H}), 2.36$ (s, 2H), $1.75(\mathrm{~s}, 6 \mathrm{H}) \mathrm{ppm}$.

${ }^{13}$ C NMR (151 MHz, CDCl3) $\delta 173.5,146.7,130.2,128.5,126.9,125.2,124.8,59.6,59.0,47.9$, 36.7, 27.9, 26.4.

HRMS (APCI) m/z: [M+H] $]^{+}$calc'd. for $\mathrm{C}_{18} \mathrm{H}_{22} \mathrm{NO}, 268.16959$, found 268.17006. 
<smiles>CN1CC2(C=CCC=C2)CC1=O</smiles>

\section{2-methyl-2-azaspiro[4.5]deca-6,9-dien-3-one (S52):}

Prepared according to General Dearomative Spirolactamization Procedure 1 using $N$-benzyl-2chloro- $N$-methylacetamide (S43) (20 mg, $0.1 \mathrm{mmol}, 1.0$ equiv), 3DPAFIPN (3.2 mg, $0.005 \mathrm{mmol}$, $5 \mathrm{~mol} \%$ ), DIPEA (52 $\mu \mathrm{L}, 0.3 \mathrm{mmol}, 3.0$ equiv), and $50 \% \mathrm{H}_{2} \mathrm{O} / \mathrm{MeCN}(2 \mathrm{~mL}, 0.05 \mathrm{M}) . \mathrm{CDCl}_{3}$ and an internal standard of dibromomethane $(7 \mu \mathrm{L}, 0.1 \mathrm{mmol})$ were added to the crude residue. The sample was analyzed by ${ }^{1} \mathrm{H}$ NMR $(\mathrm{d} 1=5 \mathrm{~s})$, and the integral values were used to calculate the yield of the title compound (12\%) and the hydrodehalogenation byproduct (93\%). ${ }^{1} \mathrm{H}$ NMR spectrum of HDH byproduct is consistent with reported values. ${ }^{26}$

\section{Characteristic peaks}

${ }^{1} \mathbf{H}$ NMR $\left(400 \mathrm{MHz} \mathrm{CDCl}_{3}\right) \delta 5.82-5.75(\mathrm{~m}, 2 \mathrm{H}), 5.66(\mathrm{dt}, \mathrm{J}=10.3,2.0 \mathrm{~Hz}, 2 \mathrm{H}), 3.23(\mathrm{~s}, 2 \mathrm{H})$, $2.84(\mathrm{t}, \mathrm{J}=0.8 \mathrm{~Hz}, 3 \mathrm{H}), 2.64(\mathrm{ddd}, \mathrm{J}=5.4,3.4,2.0 \mathrm{~Hz}, 2 \mathrm{H}), 2.35(\mathrm{~d}, \mathrm{~J}=0.9 \mathrm{~Hz}, 2 \mathrm{H}) \mathrm{ppm}$.

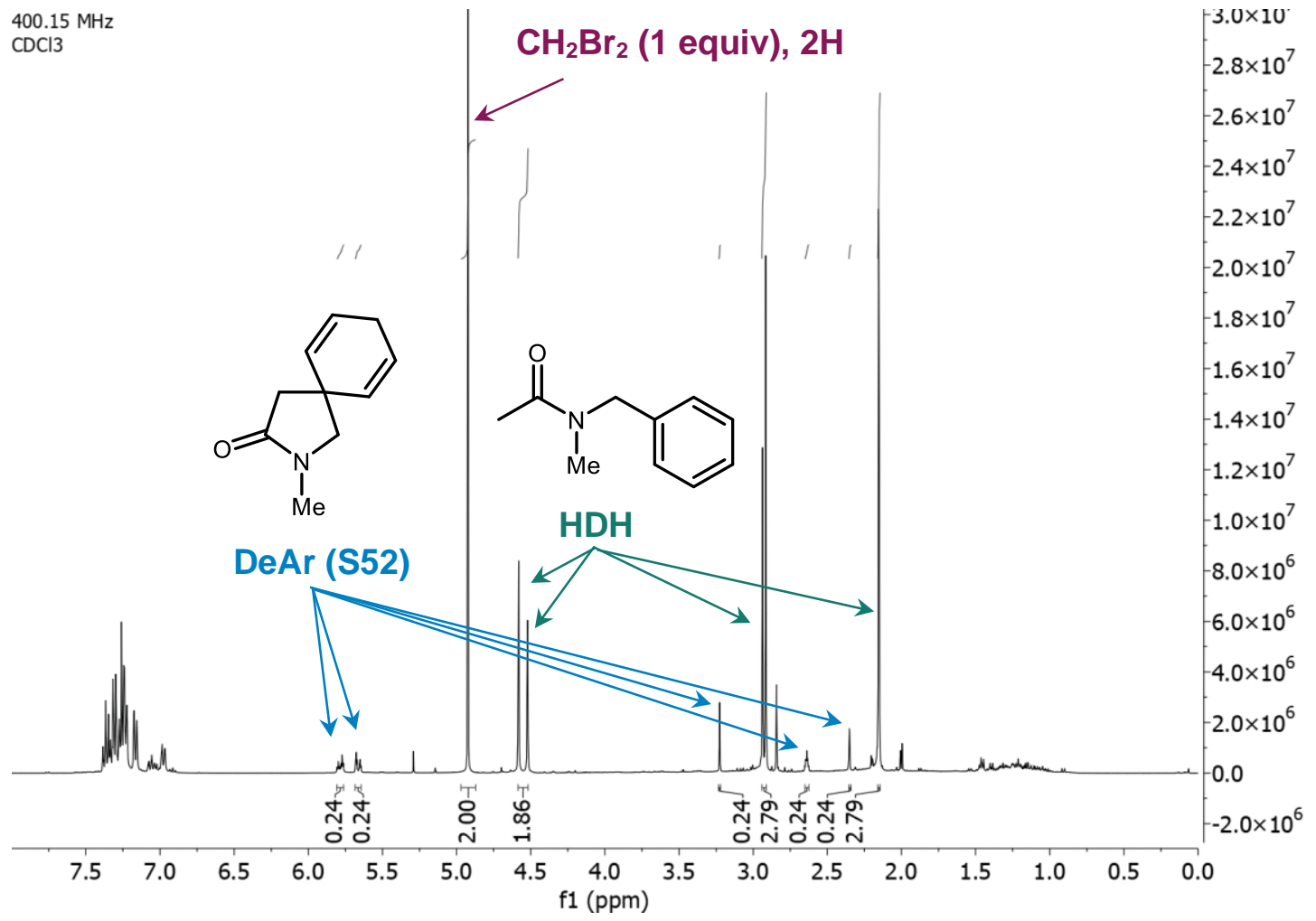

\footnotetext{
${ }^{26}$ Rauser, M.; Ascheberg, A.; Niggemann, M. Direct Reductive N-Functionalization of Aliphatic Nitro Compounds. Chem. Eur. J. 2018, 24, 3970-3974.
} 
<smiles>CC(C)N1CC2(C=CCC=C2)CC1=O</smiles>

\section{2-isopropyl-2-azaspiro[4.5]deca-6,9-dien-3-one (S53):}

Prepared according to General Dearomative Spirolactamization Procedure 1 using $N$-benzyl-2chloro- $N$-isopropylacetamide (S45) $(23 \mathrm{mg}, 0.1 \mathrm{mmol}, 1.0$ equiv), 3DPAFIPN (3.2 mg, 0.005 mmol, $5 \mathrm{~mol} \%$ ), DIPEA ( $52 \mu \mathrm{L}, 0.3 \mathrm{mmol}, 3.0$ equiv), and $50 \% \mathrm{H}_{2} \mathrm{O} / \mathrm{MeCN}(2 \mathrm{~mL}, 0.05 \mathrm{M})$. $\mathrm{CDCl}_{3}$ and an internal standard of dibromomethane $(7 \mu \mathrm{L}, 0.1 \mathrm{mmol})$ were added to the crude residue. The sample was analyzed by ${ }^{1} \mathrm{H}$ NMR $(\mathrm{d} 1=5 \mathrm{~s})$, and the integral values were used to calculate the yield of the title compound (37\%) and the hydrodehalogenation byproduct $(61 \%) .{ }^{1} \mathrm{H}$ NMR spectrum of HDH byproduct is consistent with reported values. ${ }^{27}$

\section{Characteristic peaks}

${ }^{1} \mathbf{H}$ NMR $\left(400 \mathrm{MHz}, \mathrm{CDCl}_{3}\right) \delta 5.86-5.74(\mathrm{~m}, 2 \mathrm{H}), 5.65(\mathrm{dt}, \mathrm{J}=10.4,2.0 \mathrm{~Hz}, 2 \mathrm{H}), 4.12(\mathrm{p}, \mathrm{J}=$ $6.7 \mathrm{~Hz}, 1 \mathrm{H}), 3.15$ (s, 2H), 2.65 (tt, J = 3.4, $2.0 \mathrm{~Hz}, 2 \mathrm{H}), 2.35$ (s, 2H) ppm.

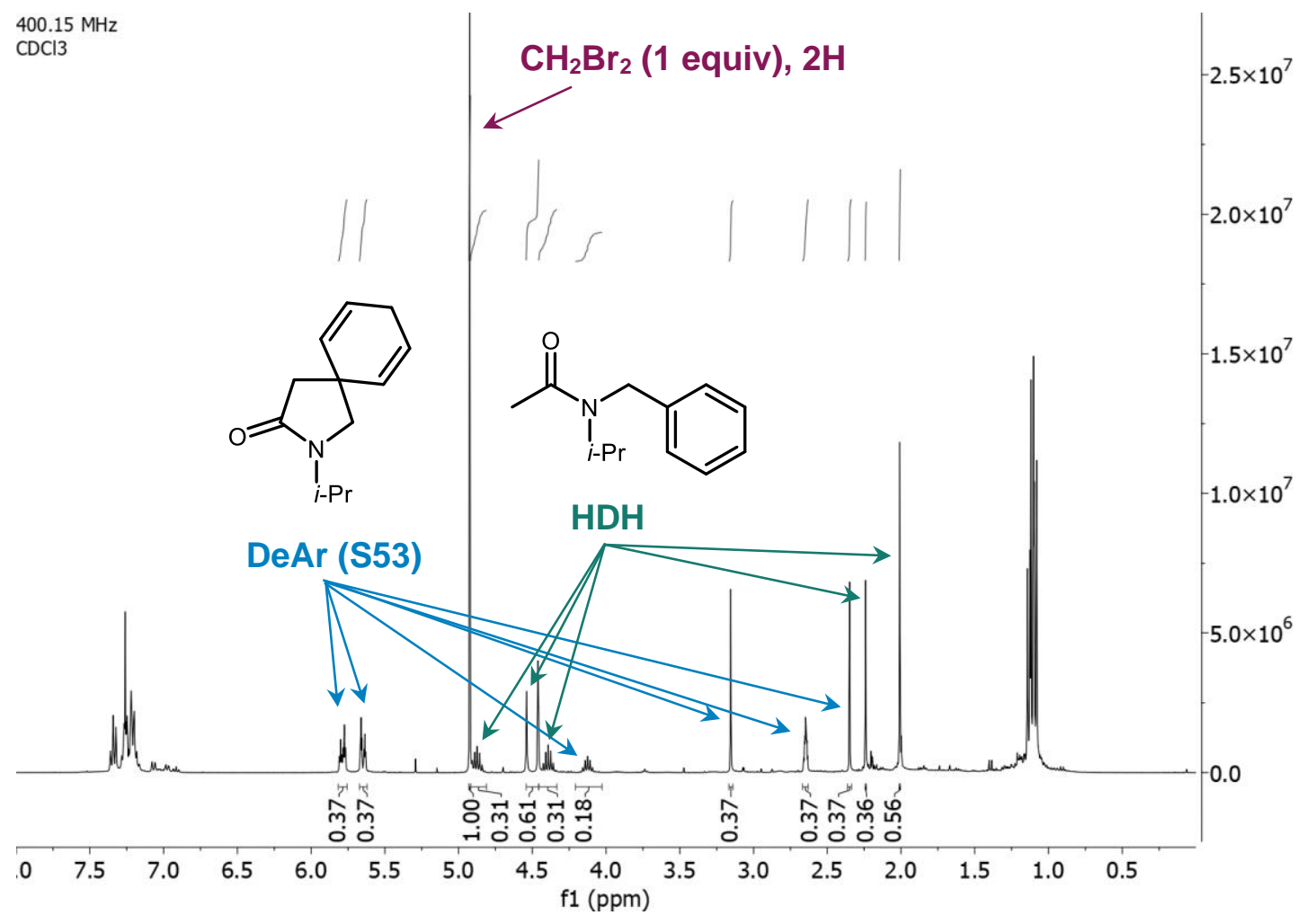

\footnotetext{
${ }^{27}$ Rauser, M.; Ascheberg, A.; Niggemann, M. Direct Reductive N-Functionalization of Aliphatic Nitro Compounds. Chem. Eur. J. 2018, 24, 3970-3974.
} 
<smiles>O=C1CC2(C=CCC=C2)CN1Cc1ccccc1</smiles>

\section{2-benzyl-2-azaspiro[4.5]deca-6,9-dien-3-one (S54):}

Prepared according to General Dearomative Spirolactamization Procedure 1 using $N, N$-dibenzyl2-chloroacetamide (S46) (27 mg, $0.1 \mathrm{mmol}, 1.0$ equiv), 3DPAFIPN (3.2 mg, $0.005 \mathrm{mmol}, 5$ mol\%), DIPEA (52 $\mu \mathrm{L}, 0.3 \mathrm{mmol}, 3.0$ equiv), and $50 \% \mathrm{H}_{2} \mathrm{O} / \mathrm{MeCN}(2 \mathrm{~mL}, 0.05 \mathrm{M}) . \mathrm{CDCl}_{3}$ and an internal standard of dibromomethane $(7 \mu \mathrm{L}, 0.1 \mathrm{mmol})$ were added to the crude residue. The sample was analyzed by ${ }^{1} \mathrm{H}$ NMR $(\mathrm{d} 1=5 \mathrm{~s})$, and the integral values were used to calculate the yield of the title compound (42\%) and the hydrodehalogenation byproduct (52\%). ${ }^{1} \mathrm{H} \mathrm{NMR}$ spectrum of $\mathrm{HDH}$ byproduct is consistent with reported values. ${ }^{28}$

\section{Characteristic peaks}

${ }^{1}$ H NMR (400 MHz, $\left.\mathrm{CDCl}_{3}\right) \delta 5.77-5.69(\mathrm{~m}, 2 \mathrm{H}), 5.61(\mathrm{dt}, \mathrm{J}=10.4,2.0 \mathrm{~Hz}, 2 \mathrm{H}), 4.45(\mathrm{~s}, 2 \mathrm{H})$, $3.10(\mathrm{~s}, 2 \mathrm{H}), 2.60(\mathrm{dtt}, \mathrm{J}=4.1,3.3,2.1 \mathrm{~Hz}, 2 \mathrm{H}), 2.42(\mathrm{~s}, 2 \mathrm{H}) \mathrm{ppm}$.

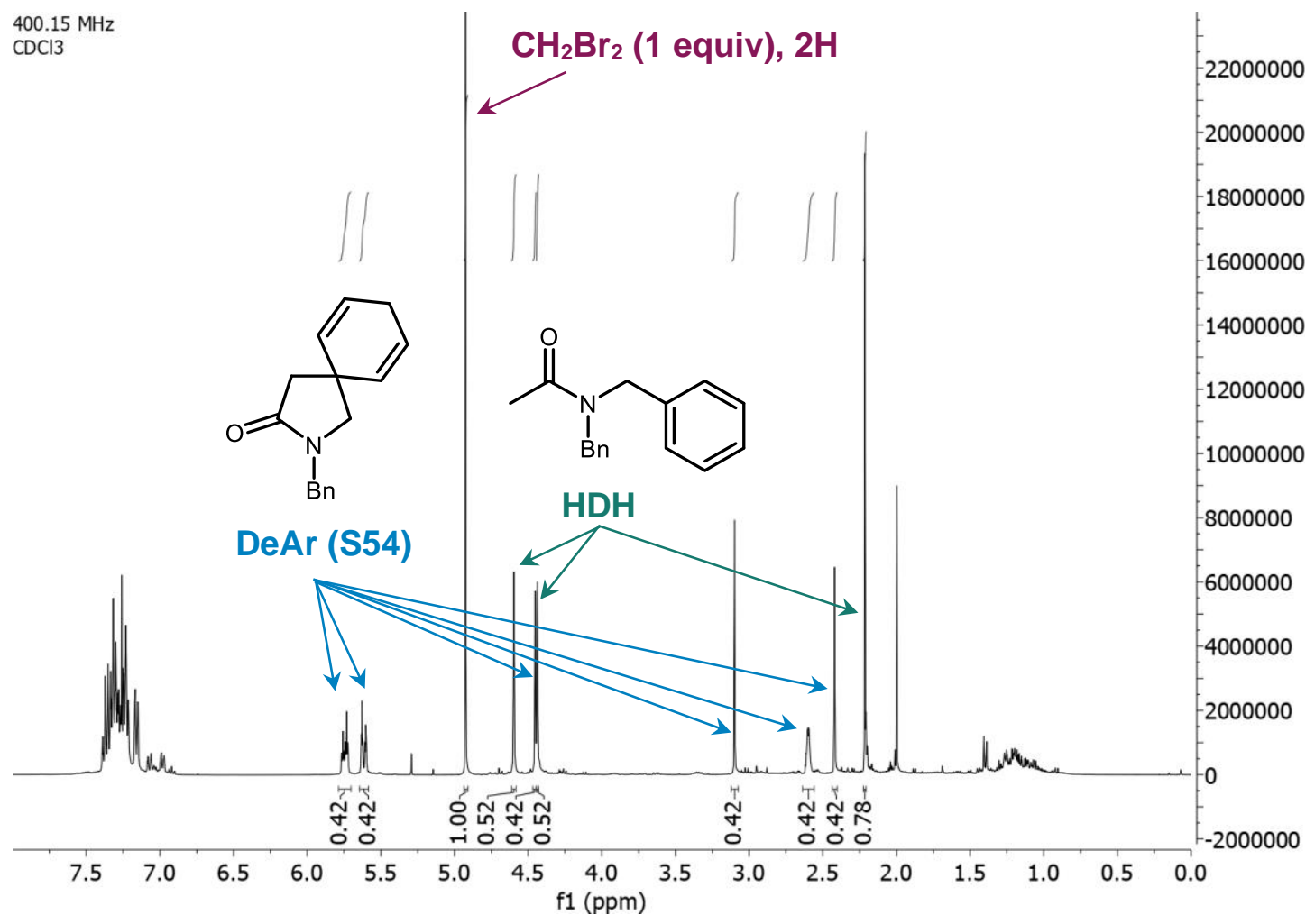

${ }^{28}$ Zhou, S.; Junge, K.; Addis, D.; Das, S.; Beller, M. A Convenient and General Iron-Catalyzed Reduction of Amides to Amines. Angew. Chem. Int. Ed. 2009, 48, 9507-9510. 
<smiles>CC(C)(C)c1cccc(CN(Cc2cccc(C(F)(F)F)c2)C(=O)CCl)c1</smiles>

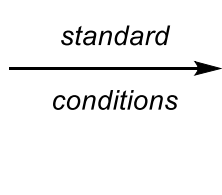

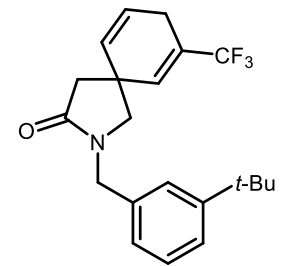

$10 \%$<smiles>CC(C)(C)C1=CC2(C=CC1)CC(=O)N(Cc1cccc(C(F)(F)F)c1)C2</smiles>

$11 \%$

2-(3-tert-butylbenzyl)-7-(trifluoromethyl)-2-azaspiro[4.5]deca-6,9-dien-3-one (S55) and 7tert-butyl-2-(3-(trifluoromethyl)benzyl)-2-azaspiro[4.5]deca-6,9-dien-3-one (S56):

Prepared according to General Dearomative Spirolactamization Procedure 1 using $\mathrm{N}$-(3-(tertbutyl)benzyl)-2-chloro- $N$-(3-(trifluoromethyl)benzyl)acetamide (S48) (39.8 mg, $0.1 \mathrm{mmol}, 1.0$ equiv), 3DPAFIPN (3.2 mg, $0.005 \mathrm{mmol}, 5 \mathrm{~mol} \%$ ), DIPEA (52 $\mu \mathrm{L}, 0.3 \mathrm{mmol}, 3.0$ equiv), and $50 \% \mathrm{H}_{2} \mathrm{O} / \mathrm{MeCN}(2 \mathrm{~mL}, 0.05 \mathrm{M}) . \mathrm{CDCl}_{3}$ and an internal standard of dibromomethane $(7 \mu \mathrm{L}, 0.1$ mmol) were added to the crude residue. The sample was analyzed by ${ }^{1} \mathrm{H}$ NMR $(\mathrm{d} 1=5 \mathrm{~s})$, and the integral values were used to calculate the yield of the title compounds $(10 \%, 11 \%)$ and the hydrodehalogenation byproduct (74\%).

S55 (characteristic peaks):

${ }^{1} \mathbf{H}$ NMR (400 MHz, $\left.\mathrm{CDCl}_{3}\right) \delta 6.26-6.18(\mathrm{~m}, 1 \mathrm{H}), 2.81-2.74(\mathrm{~m}, 2 \mathrm{H}) \mathrm{ppm}$.

S56 (characteristic peaks):

${ }^{\mathbf{1}} \mathbf{H}$ NMR $\left(400 \mathrm{MHz}, \mathrm{CDCl}_{3}\right) \delta 5.41$ (q, J = 1.7 Hz, 1H), $2.65(\mathrm{tt}, \mathrm{J}=3.8,1.9 \mathrm{~Hz}, 2 \mathrm{H}) \mathrm{ppm}$.

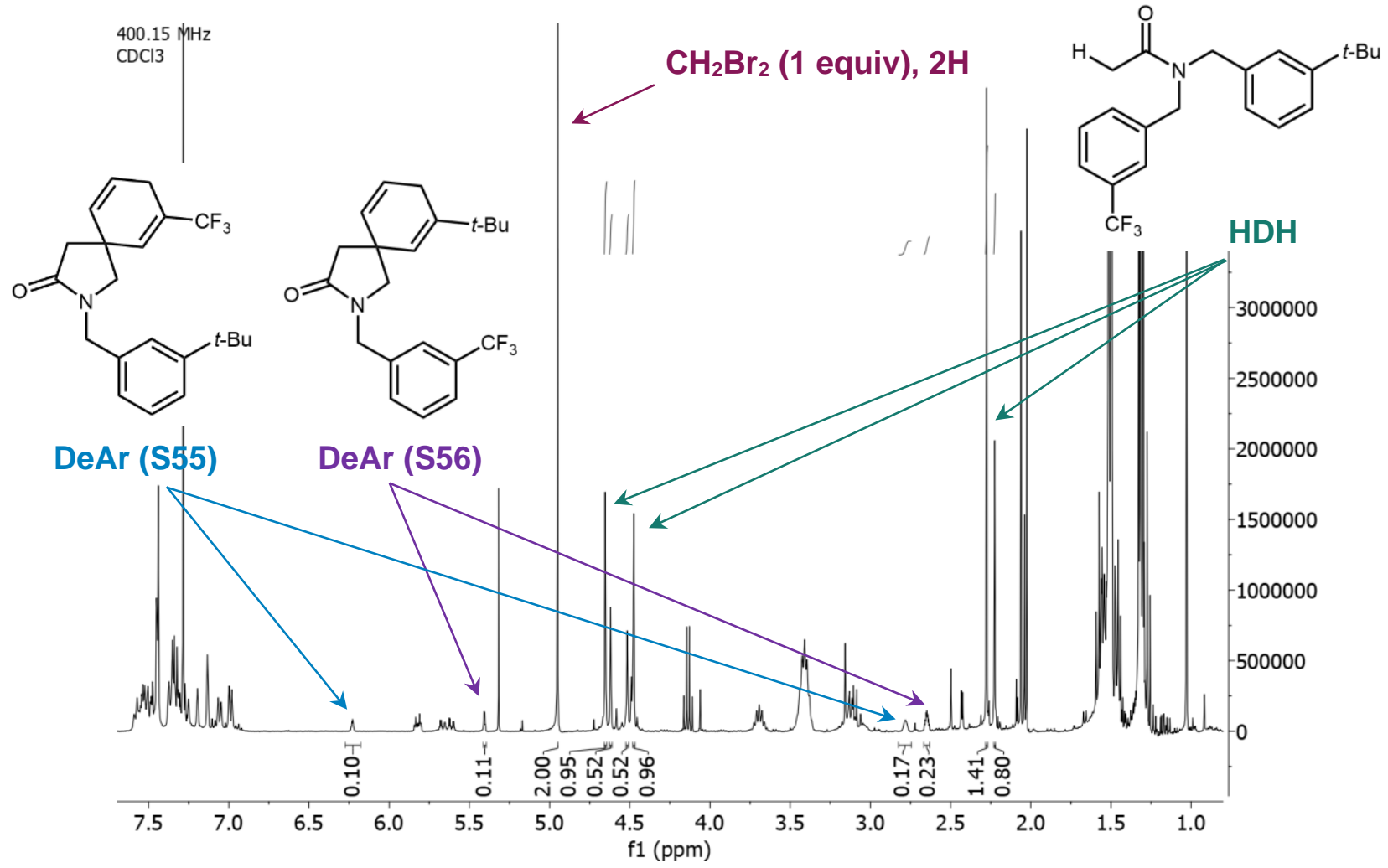


<smiles>O=C1N(Cc2ccccc2)CC12C=CC(C1C=CC3(C=C1)CN(Cc1ccccc1)C(=O)C3(F)F)C=C2</smiles>

2,2'-dibenzyl-4,4,4',4' 'tetrafluoro-2,2'-diaza[8,8' -bispiro[4.5]decane-6,6' ,9,9' '-tetraene]-3,3'dione (S57):

Prepared according to General Dearomative Spirolactamization Procedure 1 using $N, N$-dibenzyl2-bromo-2,2-difluoroacetamide (S49) (35.4 mg, $0.1 \mathrm{mmol}, 1.0$ equiv), 3DPAFIPN (3.2 mg, 0.005 mmol, $5 \mathrm{~mol} \%$ ), DIPEA ( $52 \mu \mathrm{L}, 0.3 \mathrm{mmol}, 3.0$ equiv), and $50 \% \mathrm{H}_{2} \mathrm{O} / \mathrm{MeCN}(2 \mathrm{~mL}, 0.05 \mathrm{M})$. $\mathrm{CDCl}_{3}$ and an internal standard of dibromomethane $(7 \mu \mathrm{L}, 0.1 \mathrm{mmol})$ were added to the crude residue. The sample was analyzed by ${ }^{1} \mathrm{H}$ NMR $(\mathrm{d} 1=5 \mathrm{~s})$, and the integral values were used to calculate the yield of the title compound $(98 \%)$.

$399.75 \mathrm{MHz}$

cdcl3
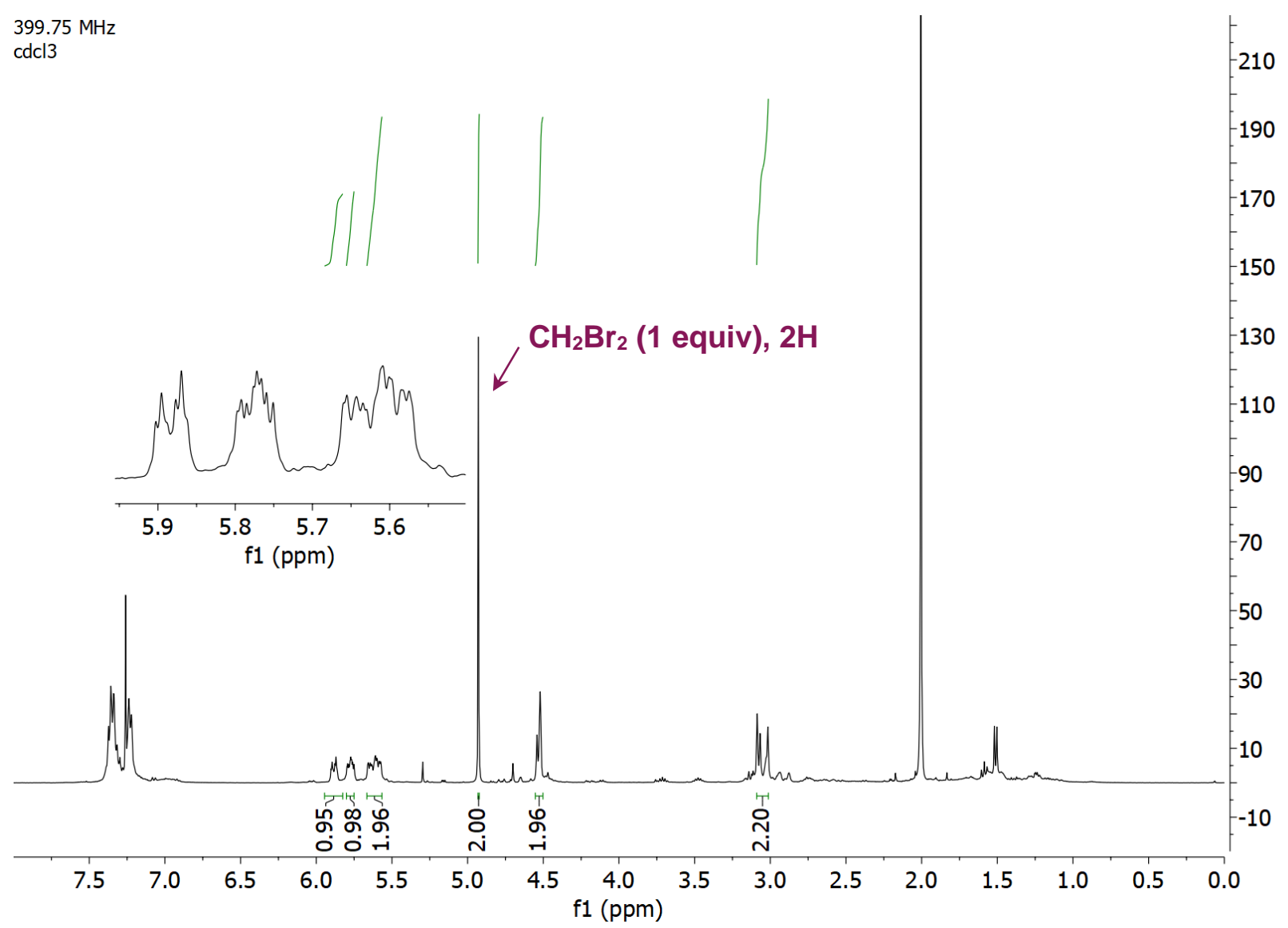
<smiles></smiles>

2,2'-dibenzyl-2,2'-diaza[8,8'-bispiro[4.5]decane-6,6',9,9'-tetraene]-3,3'-dione (S58):

Prepared according to General Dearomative Spirolactamization Procedure 1 using $N, N$-dibenzyl2,2,2-trichloroacetamide (S50) (34.3 mg, $0.1 \mathrm{mmol}, 1.0$ equiv), 3DPAFIPN ( $3.2 \mathrm{mg}, 0.005 \mathrm{mmol}$, $5 \mathrm{~mol} \%$ ), DIPEA ( $52 \mu \mathrm{L}, 0.3 \mathrm{mmol}, 3.0$ equiv), and $50 \% \mathrm{H}_{2} \mathrm{O} / \mathrm{MeCN}(2 \mathrm{~mL}, 0.05 \mathrm{M}) . \mathrm{CDCl}_{3}$ and an internal standard of dibromomethane $(7 \mu \mathrm{L}, 0.1 \mathrm{mmol})$ were added to the crude residue. The sample was analyzed by ${ }^{1} \mathrm{H}$ NMR $(\mathrm{d} 1=5 \mathrm{~s})$, and the integral values were used to calculate the yield of the title compound (81\%).

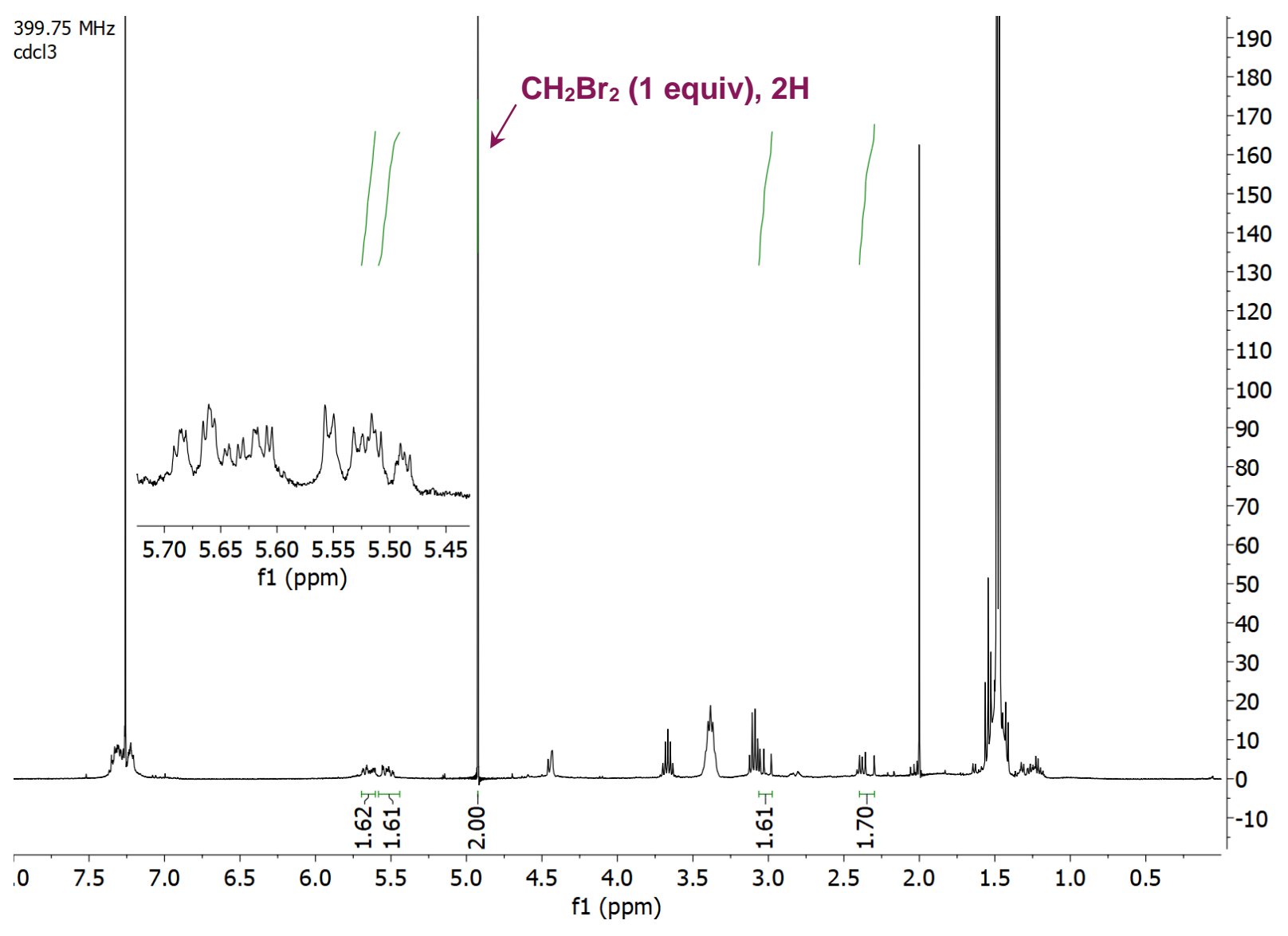


VIII-A. Derivatization

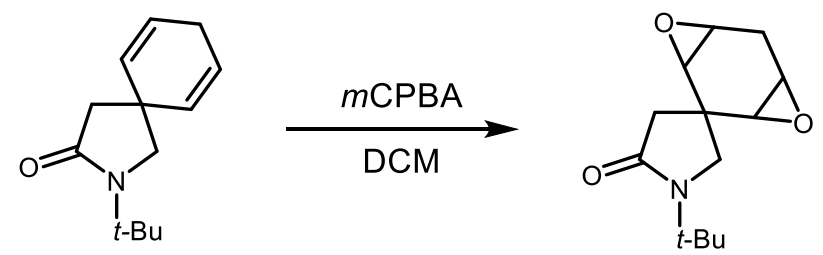

1-(tert-butyl)-4',8'-dioxaspiro[pyrrolidine-3,2'-tricyclo[5.1.0.0 ${ }^{3,5}$ ]octan]-5-one (20):

To an reaction vial charged with 2-(tert-butyl)-2-azaspiro[4.5]deca-6,9-dien-3-one (19) (62 mg, $0.3 \mathrm{mmol}, 1.0$ equiv) cooled to $0{ }^{\circ} \mathrm{C}$ was added $\mathrm{CH}_{2} \mathrm{Cl}_{2}(6 \mathrm{~mL}, 0.05 \mathrm{M})$ and $m \mathrm{CPBA}(75 \%, 172$ $\mathrm{mg}, 0.75 \mathrm{mmol}, 2.5$ equiv). The resulting solution was allowed to stir at $23{ }^{\circ} \mathrm{C}$ for 17 hours. The precipitate was filtered and washed with $\mathrm{CH}_{2} \mathrm{Cl}_{2}$ and $1 \mathrm{M} \mathrm{NaOH}$ (aq). The filtrate was extracted with $\mathrm{CH}_{2} \mathrm{Cl}_{2}(3 \mathrm{x})$, washed with brine, dried over $\mathrm{MgSO}_{4}$, and concentrated under reduced pressure. The crude residue was purified on silica gel (10-50\% acetone/hexanes) to yield the title compound (57.0 mg, 82\%, 5:6:1 d.r.; diastereomers 2 and 3 isolated as a mixture).<smiles>CC(C)(C)N1CC2(CC1=O)C1OC1CC1OC12</smiles>

Diastereomer 1 (elutes first, $23.8 \mathrm{mg}$, white amorphous solid): (1'R,3'R,5'S,7'S)-1-(tert-butyl)4',8'-dioxaspiro[pyrrolidine-3,2'-tricyclo[5.1.0.0 ${ }^{3,5}$ ]octan]-5-one:

Rf: 0.55 (50\% acetone/hexanes)

${ }^{1} \mathbf{H}$ NMR (400 MHz, $\left.\mathrm{CDCl}_{3}\right) \delta 3.68(\mathrm{~d}, \mathrm{~J}=10.5 \mathrm{~Hz}, 1 \mathrm{H}), 3.37(\mathrm{~d}, \mathrm{~J}=10.5 \mathrm{~Hz}, 1 \mathrm{H}), 3.18(\mathrm{tq}, \mathrm{J}=$ 4.0, $1.8 \mathrm{~Hz}, 2 \mathrm{H}), 2.94(\mathrm{dt}, \mathrm{J}=5.8,2.9 \mathrm{~Hz}, 2 \mathrm{H}), 2.71(\mathrm{~d}, \mathrm{~J}=17.0 \mathrm{~Hz}, 1 \mathrm{H}), 2.42$ (d, J = 16.9 Hz, $1 \mathrm{H}), 2.34(\mathrm{t}, \mathrm{J}=2.3 \mathrm{~Hz}, 2 \mathrm{H}), 1.43(\mathrm{~s}, 9 \mathrm{H}) \mathrm{ppm}$.

${ }^{13} \mathrm{C}$ NMR $\left(151 \mathrm{MHz}, \mathrm{CDCl}_{3}\right) \delta 172.8,56.5,55.9,54.5,52.4,51.0,50.8,41.6,34.8,27.9,23.9$ ppm.

HRMS (APCI) m/z: [M+H] $]^{+}$calc'd. for $\mathrm{C}_{13} \mathrm{H}_{20} \mathrm{NO}_{3}, 238.14377$, found 238.14375.<smiles>CC(C)(C)N1CC2(CC1=O)C1OC1CC1OC12</smiles>

Diastereomer 2 (elutes second, $29.0 \mathrm{mg}$, white amorphous solid): (1'R,3r,3'S,5'R,7'S)-1-(tertbutyl)-4', $8^{\prime}$-dioxaspiro[pyrrolidine-3,2'-tricyclo[5.1.0.0 $\left.{ }^{3,5}\right]$ octan]-5-one one:

Rf: 0.35 (50\% acetone/hexanes)

${ }^{1} \mathbf{H}$ NMR (400 MHz, $\left.\mathrm{CDCl}_{3}\right) \delta 3.78(\mathrm{~s}, 2 \mathrm{H}), 3.23$ (ddd, J = 4.1, 2.9, $\left.1.2 \mathrm{~Hz}, 2 \mathrm{H}\right), 3.05-3.01(\mathrm{~m}$, 2H), $2.76(\mathrm{dt}, \mathrm{J}=17.3,1.3 \mathrm{~Hz}, 1 \mathrm{H}), 2.43(\mathrm{~s}, 2 \mathrm{H}), 2.24(\mathrm{dt}, \mathrm{J}=17.3,3.0 \mathrm{~Hz}, 1 \mathrm{H}), 1.44(\mathrm{~s}, 9 \mathrm{H}) \mathrm{ppm}$. ${ }^{13}$ C NMR $\left(151 \mathrm{MHz}, \mathrm{CDCl}_{3}\right) \delta 172.0,55.8,54.7,53.1,51.3,41.0,34.4,27.9,23.0$ ppm.

HRMS (APCI) m/z: [M+H] $]^{+}$calc'd. for $\mathrm{C}_{13} \mathrm{H}_{20} \mathrm{NO}_{3}, 238.14377$, found 238.14384. 
<smiles>CC(C)(C)N1C[C@]2(CC1=O)[C@@H]1O[C@H]1C[C@@H]1O[C@@H]12</smiles>

Diastereomer 3 (elutes second, $5.1 \mathrm{mg}$, white amorphous solid): (1'R,3s,3'S,5'R,7'S)-1-(tertbutyl)-4',8'-dioxaspiro[pyrrolidine-3,2'-tricyclo[5.1.0.0 ${ }^{3,5}$ ]octan]-5-one one (characteristic peaks):

Rf: 0.35 (50\% acetone/hexanes)

${ }^{1} \mathbf{H}$ NMR $\left(400 \mathrm{MHz} \mathrm{CDCl}_{3}\right) \delta 3.43(\mathrm{~s}, 2 \mathrm{H}), 3.20(\mathrm{ddd}, \mathrm{J}=3.9,2.9,1.2 \mathrm{~Hz}, 2 \mathrm{H}), 3.01$ - 2.99 (m, 2H), $2.79(\mathrm{~s}, 2 \mathrm{H}), 2.21(\mathrm{dt}, \mathrm{J}=17.2,3.0 \mathrm{~Hz}, 2 \mathrm{H}), 1.43(\mathrm{~s}, 9 \mathrm{H}) \mathrm{ppm}$.

${ }^{13} \mathrm{C}$ NMR $\left(151 \mathrm{MHz}, \mathrm{CDCl}_{3}\right) \delta 172.8,56.0,54.5,52.0,41.6,34.2,27.9 \mathrm{ppm}$.

HRMS (APCI) m/z: $[\mathrm{M}+\mathrm{H}]^{+}$calc'd. for $\mathrm{C}_{13} \mathrm{H}_{20} \mathrm{NO}_{3}, 238.14377$, found 238.14384.<smiles>CC(C)(C)N1CC2(C=CCC=C2)CC1=O</smiles>
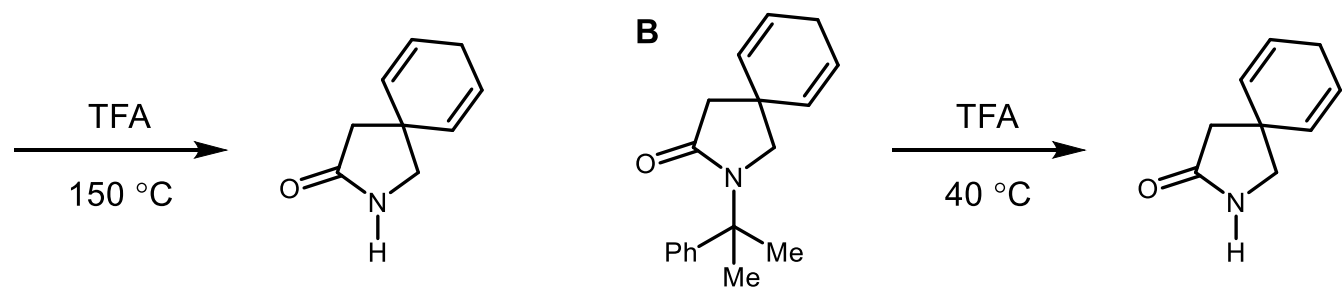

2-azaspiro[4.5]deca-6,9-dien-3-one (21):

Procedure A: Dissolved 2-(tert-butyl)-2-azaspiro[4.5]deca-6,9-dien-3-one (19) (20.5 mg, 0.1 mmol, 1.0 equiv) in TFA $(4 \mathrm{~mL})$. The reaction was heated to $150{ }^{\circ} \mathrm{C}$ in a pressure tube. After 16 hours, quenched with $1 \mathrm{M} \mathrm{NaOH}(\mathrm{aq})$, extracted with $\mathrm{CH}_{2} \mathrm{Cl}_{2}(3 \mathrm{x})$ and concentrated under reduced pressure. The crude residue was purified on silica gel (10-100\% ethyl acetate/hexanes) to yield the title compound as an off-white amorphous solid (14.9 mg, 100\%).

Procedure B: Dissolved 2-(2-phenylpropan-2-yl)-2-azaspiro[4.5]deca-6,9-dien-3-one (S51) (57.8 $\mathrm{mg}, 0.22 \mathrm{mmol}, 1.0$ equiv) in TFA $(1 \mathrm{~mL})$. The reaction was heated to $40{ }^{\circ} \mathrm{C}$. After 2 hours, quenched with $1 \mathrm{M} \mathrm{NaOH}(\mathrm{aq})$, extracted with $\mathrm{CH}_{2} \mathrm{Cl}_{2}(3 \mathrm{x})$ and concentrated under reduced pressure. The crude residue was purified on silica gel (20-100\% ethyl acetate/hexanes) to yield the title compound as an off-white amorphous solid (19.0 mg, 59\%).

Rf: 0.29 (100\% EtOAc)

${ }^{1}$ H NMR (600 MHz, CDCl3) $\delta 6.55$ (s, 1H), $5.78(\mathrm{dt}, \mathrm{J}=10.4,3.3 \mathrm{~Hz}, 2 \mathrm{H}), 5.72$ (dt, J = 10.4, 2.0 $\mathrm{Hz}, 2 \mathrm{H}), 3.24$ (s, 2H), 2.64 (tt, J = 3.5, $1.9 \mathrm{~Hz}, 2 \mathrm{H}), 2.28$ (s, 2H) ppm.

${ }^{13}$ C NMR 13C NMR (151 MHz, CDCl3) $\delta$ 177.3, 130.1, 124.7, 55.4, 45.3, 39.9, 26.3 ppm.

HRMS (APCI) m/z: $[\mathrm{M}+\mathrm{H}]^{+}$calc'd. for $\mathrm{C}_{9} \mathrm{H}_{12} \mathrm{NO}, 150.09134$, found 150.09157 .<smiles>CC(C)(C)N1CC2(C=CCC=C2)CC1C(C)(C)C(C)(C)C</smiles>

2-(tert-butyl)-2-azaspiro[4.5]deca-6,9-diene (22):

To an oven-dried reaction vial charged with 2-(tert-butyl)-2-azaspiro[4.5]deca-6,9-dien-3-one (19) (20.5 mg, $0.1 \mathrm{mmol}, 1.0$ equiv) was added THF $(1 \mathrm{~mL}, 0.1 \mathrm{M})$. The solution was cooled to $0{ }^{\circ} \mathrm{C}$ and $\mathrm{LiAlH}_{4}(1 \mathrm{M}$ in THF, $0.25 \mathrm{~mL}, 0.25 \mathrm{mmol}, 2.5$ equiv) was added dropwise. The resulting 
solution was allowed to warm to $23{ }^{\circ} \mathrm{C}$ and stir for 20 hours. The reaction was cooled to $0{ }^{\circ} \mathrm{C}$ and diluted with $\mathrm{Et}_{2} \mathrm{O}$. Then $0.01 \mathrm{~mL} \mathrm{H}_{2} \mathrm{O}, 0.01 \mathrm{~mL} 15 \% \mathrm{NaOH}(\mathrm{aq})$, and $0.03 \mathrm{~mL} \mathrm{H}_{2} \mathrm{O}$ were added sequentially, and the resulting solution was stirred for 15 minutes at $23{ }^{\circ} \mathrm{C}$. Anhydrous $\mathrm{MgSO}_{4}$ was added and the resulting suspension was stirred for an additional 15 minutes. After filtration and concentration under reduced pressure, the title compound was obtained as a white amorphous solid (19.1 mg, 100\%).

Rf: 0.19 (5\% MeOH/DCM)

${ }^{1} \mathbf{H}$ NMR $\left(600 \mathrm{MHz}, \mathrm{C}_{6} \mathrm{D}_{6}\right) \delta 5.83(\mathrm{dt}, \mathrm{J}=10.4,2.1 \mathrm{~Hz}, 2 \mathrm{H}), 5.60(\mathrm{dt}, \mathrm{J}=10.2,3.3 \mathrm{~Hz}, 2 \mathrm{H}), 2.66$ $(\mathrm{t}, \mathrm{J}=7.0 \mathrm{~Hz}, 2 \mathrm{H}), 2.63(\mathrm{~s}, 2 \mathrm{H}), 2.49(\mathrm{tt}, \mathrm{J}=3.5,2.1 \mathrm{~Hz}, 2 \mathrm{H}), 1.75(\mathrm{t}, \mathrm{J}=7.0 \mathrm{~Hz}, 2 \mathrm{H}), 1.00(\mathrm{~s}, 9 \mathrm{H})$ ppm.

${ }^{13}$ C NMR $\left(151 \mathrm{MHz}, \mathrm{C}_{6} \mathrm{D}_{6}\right) \delta$ 134.2, 122.0, 60.8, 52.0, 45.6, 42.1, 41.3, 26.8, $26.2 \mathrm{ppm}$.

HRMS (APCI) m/z: [M+H] $]^{+}$calc'd. for $\mathrm{C}_{13} \mathrm{H}_{22} \mathrm{~N}, 192.17468$, found 192.17484 .

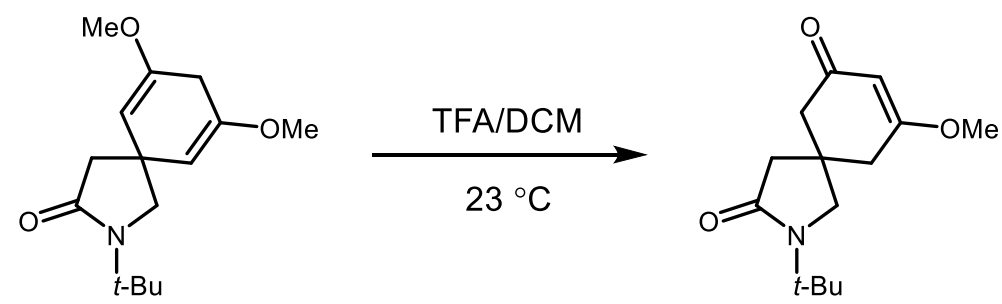

2-(tert-butyl)-9-methoxy-2-azaspiro[4.5]dec-8-ene-3,7-dione (24):

Dissolved 2-(tert-butyl)-7,9-dimethoxy-2-azaspiro[4.5]deca-6,9-dien-3-one (7) (2.8 mg, 0.011 mmol) in $\mathrm{CH}_{2} \mathrm{Cl}_{2}(0.5 \mathrm{~mL})$ and added TFA $(0.1 \mathrm{~mL})$. After 18 hours, quenched with saturated $\mathrm{NaHCO}_{3}(\mathrm{aq})$, extracted with EtOAc (3x) and concentrated under reduced pressure to obtain the title compound as a yellow oil (2.7 $\mathrm{mg}, 100 \%)$.

Rf: 0.14 (20\% EtOAc/hexanes)

${ }^{1} \mathbf{H}$ NMR $\left(400 \mathrm{MHz}, \mathrm{CDCl}_{3}\right) \delta 5.48(\mathrm{~s}, 1 \mathrm{H}), 3.75(\mathrm{~s}, 3 \mathrm{H}), 3.34(\mathrm{~s}, 2 \mathrm{H}), 2.60-2.43(\mathrm{~m}, 4 \mathrm{H}), 2.47$ (s, 2H), 1.39 (s, 9H) ppm.

${ }^{13} \mathrm{C}$ NMR $\left(151 \mathrm{MHz}, \mathrm{CDCl}_{3}\right) \delta 197.6,176.6,174.2,102.2,56.7,56.5,55.3,47.2,44.9,40.0,36.2$, $27.9 \mathrm{ppm}$.

HRMS (APCI) m/z: [M+H] ${ }^{+}$calc'd. for $\mathrm{C}_{14} \mathrm{H}_{22} \mathrm{NO}_{3}, 252.15942$, found 252.15975.

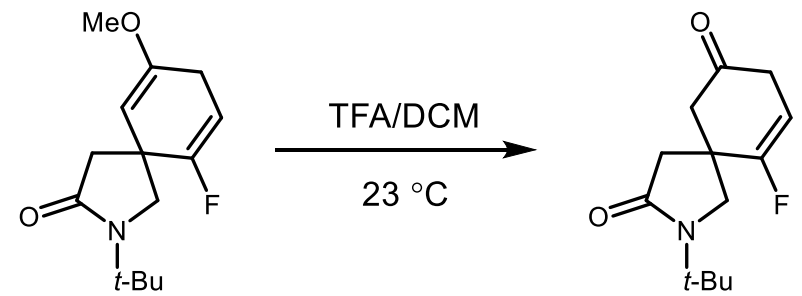

2-(tert-butyl)-10-fluoro-2-azaspiro[4.5]dec-9-ene-3,7-dione (25):

Dissolved 2-(tert-butyl)-6-fluoro-9-methoxy-2-azaspiro[4.5]deca-6,9-dien-3-one (18) (6.0 mg, $0.024 \mathrm{mmol})$ in $\mathrm{CH}_{2} \mathrm{Cl}_{2}(1.0 \mathrm{~mL})$ and added TFA $(0.2 \mathrm{~mL})$. After 16 hours, quenched with $1 \mathrm{M}$ $\mathrm{NaOH}(\mathrm{aq})$, extracted with DCM (3x) and concentrated under reduced pressure to obtain the title compound as a yellow oil (5.7 $\mathrm{mg}, 100 \%)$.

Rf: 0.58 (100\% EtOAc)

${ }^{1} \mathbf{H}$ NMR (400 MHz, CDCl3) $\delta 5.40(\mathrm{dt}, \mathrm{J}=15.5,3.9 \mathrm{~Hz}, 1 \mathrm{H}), 3.58(\mathrm{dt}, \mathrm{J}=10.0,0.8 \mathrm{~Hz}, 1 \mathrm{H}), 3.21$ (dt, J = 10.1, 0.6 Hz, 1H), 3.05 (ddd, J = 20.9, 5.5, 3.6 Hz, 1H), 2.93 (d, J = 16.6 Hz, 1H), 2.89 (dt, $\mathrm{J}=20.9,4.6 \mathrm{~Hz}, 1 \mathrm{H}), 2.77-2.62(\mathrm{~m}, 2 \mathrm{H}), 2.19(\mathrm{~d}, \mathrm{~J}=16.6 \mathrm{~Hz}, 1 \mathrm{H}), 1.38(\mathrm{~s}, 9 \mathrm{H}) \mathrm{ppm}$. 
${ }^{13} \mathbf{C}$ NMR $(101 \mathrm{MHz}, \mathrm{CDCl} 3) \delta 204.6\left(\mathrm{~d},{ }^{4} \mathrm{~J}_{\mathrm{C}-\mathrm{F}}=2.5 \mathrm{~Hz}\right), 171.8,158.6\left(\mathrm{~d},{ }^{1} \mathrm{~J}_{\mathrm{C}-\mathrm{F}}=260.1 \mathrm{~Hz}\right), 101.8$ $\left(\mathrm{d},{ }^{2} \mathrm{~J}_{\mathrm{C}-\mathrm{F}}=22.2 \mathrm{~Hz}\right), 54.6,53.4\left(\mathrm{~d},{ }^{3} \mathrm{~J}_{\mathrm{C}-\mathrm{F}}=1.5 \mathrm{~Hz}\right), 50.0\left(\mathrm{~d},{ }^{3} \mathrm{~J}_{\mathrm{C}-\mathrm{F}}=5.3 \mathrm{~Hz}\right), 42.2\left(\mathrm{~d},{ }^{3} \mathrm{~J}_{\mathrm{C}-\mathrm{F}}=2.5 \mathrm{~Hz}\right)$, $40.6\left(\mathrm{~d},{ }^{2} \mathrm{~J}_{\mathrm{C}-\mathrm{F}}=23.9 \mathrm{~Hz}\right), 37.4\left(\mathrm{~d},{ }^{3} \mathrm{~J}_{\mathrm{C}-\mathrm{F}}=8.1 \mathrm{~Hz}\right), 27.8 \mathrm{ppm}$.

HRMS (APCI) m/z: [M+H] ${ }^{+}$calc'd. for $\mathrm{C}_{13} \mathrm{H}_{19} \mathrm{FNO}_{2}, 240.13943$, found 240.13983 .<smiles>CN1CC2(C=CCC=C2)CC1=O</smiles>

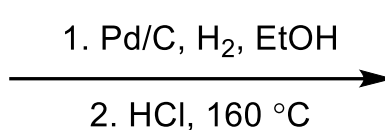<smiles>NCC1(CC(=O)O)CCCCC1</smiles>

\section{2-(1-(aminomethyl)cyclohexyl)acetic acid hydrochloride (23):}

Dissolved 2-(tert-butyl)-2-azaspiro[4.5]deca-6,9-dien-3-one (19) (102.6 mg, $0.50 \mathrm{mmol}, 1.0$ equiv) in methanol $(20 \mathrm{~mL}, 0.025 \mathrm{M})$. Added palladium (5\% on carbon, wet basis, $5 \mathrm{mg}$ ), and evacuated and backfilled with hydrogen. After 16 hours, the reaction mixture was filtered through a plug of celite to afford a white solid, which was added to a microwave vial with hydrochloric acid $\left(37 \%\right.$ in water, $5 \mathrm{~mL}$ ), and microwaved at $160^{\circ} \mathrm{C}$ for 16 hours. The resulting reaction mixture was extracted with $\mathrm{CH}_{2} \mathrm{Cl}_{2}(4 \mathrm{x})$ and $\mathrm{Et}_{2} \mathrm{O}(1 \mathrm{x})$. The aqueous phase was azeotroped with acetonitrile under reduced pressure. The resulting white solid was sonicated in $\mathrm{Et}_{2} \mathrm{O}$ and vacuum filtered to afford the title compound as a white solid (103 mg, 52\%).

${ }^{1}$ H NMR (600 MHz, D2O) $\delta 3.12$ (s, 2H), 2.56 (s, 2H), $1.57-1.35$ (m, 10H) ppm.

${ }^{1} \mathrm{H}$ NMR spectrum is consistent with reported values. ${ }^{29}$

${ }^{29}$ Nagatomo, M.; Nishiyama, H.; Fujino, M. Angew. Chem. Int. Ed. 2015, 54, 1537-1541. 


\section{NMR Spectra}
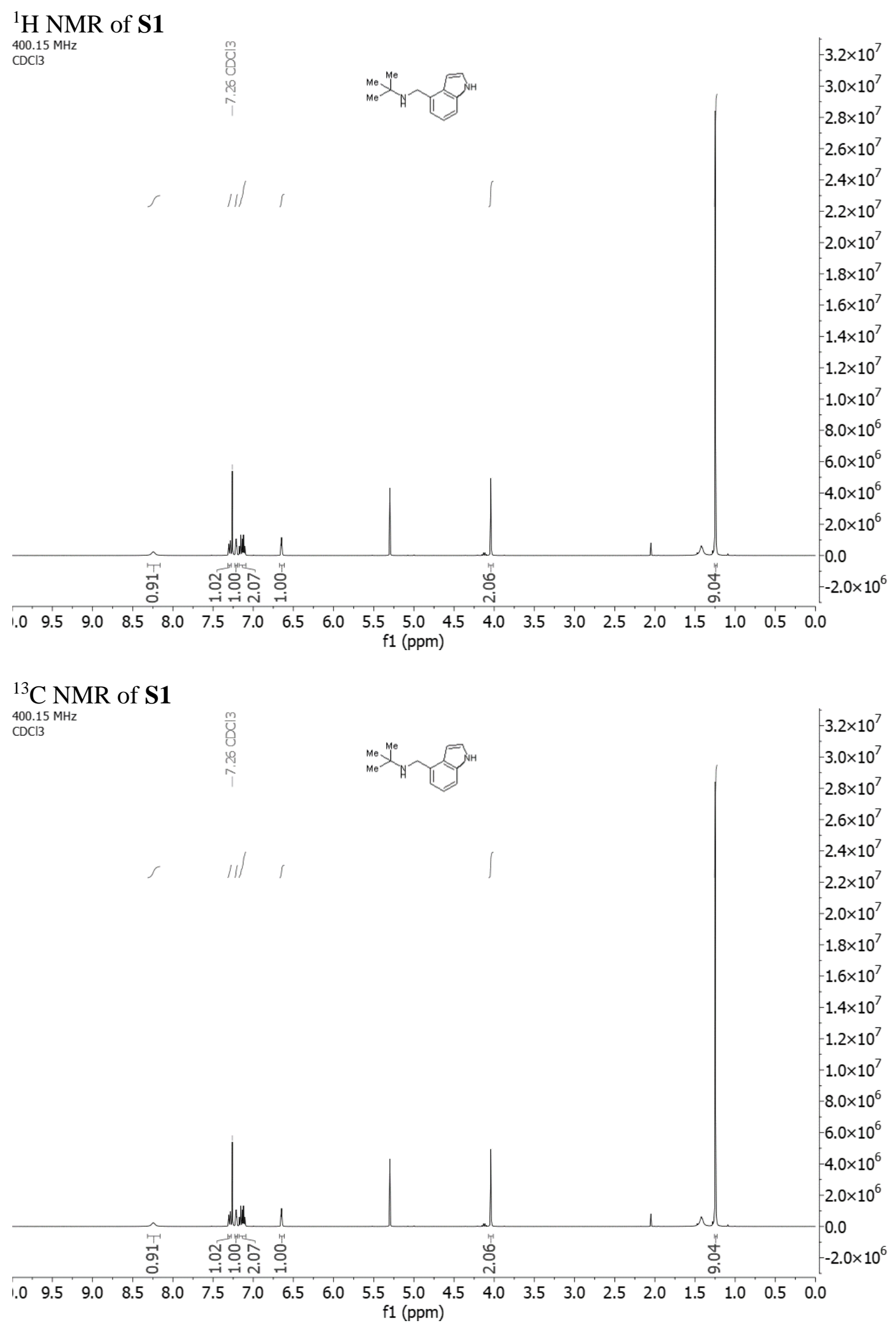
${ }^{1} \mathrm{H}$ NMR of $\mathbf{S 2}$ $400.15 \mathrm{MHz}$
$\mathrm{CDCl} 3$

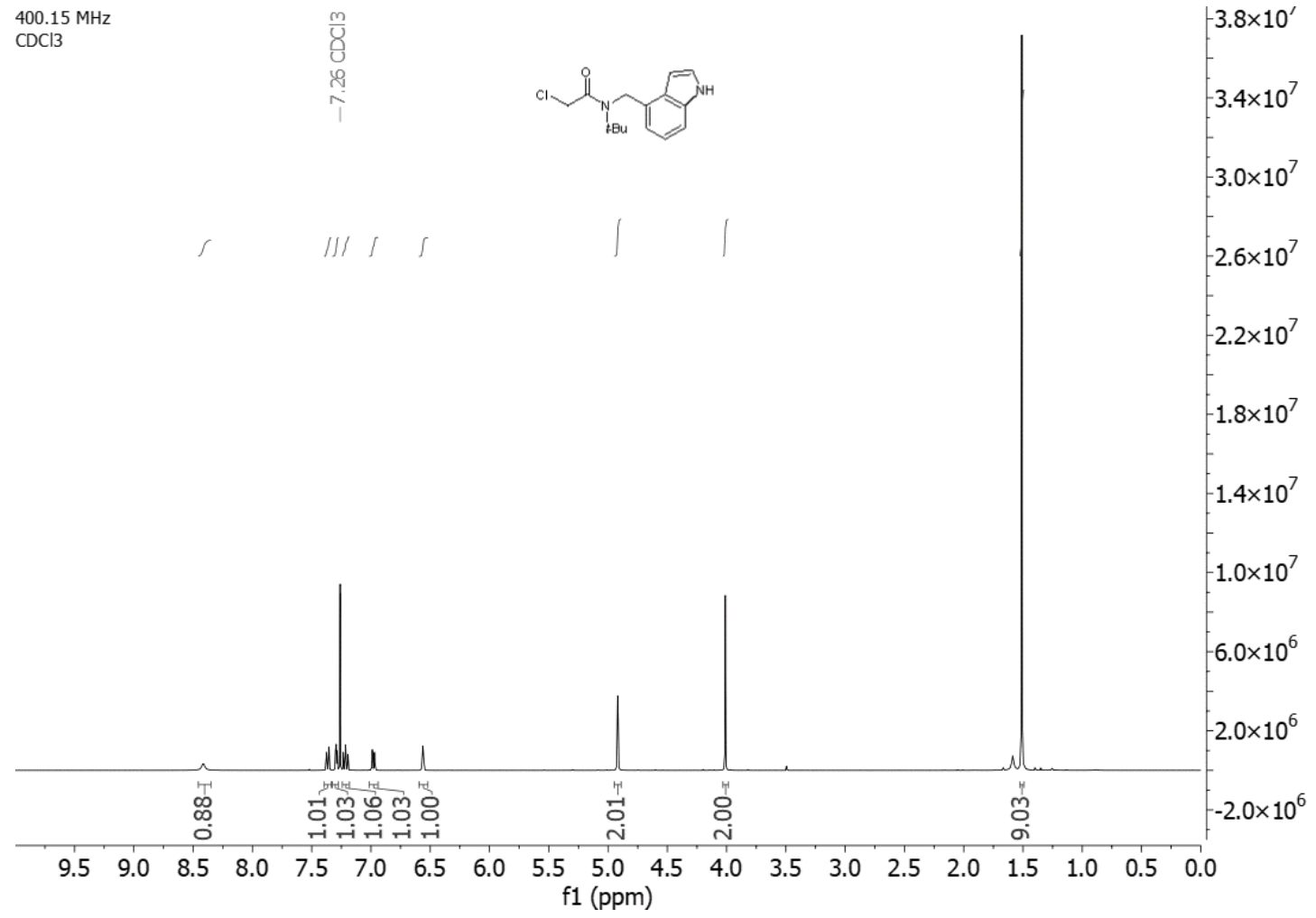

${ }^{13} \mathrm{C}$ NMR of $\mathbf{S 2}$

$150.93 \mathrm{MHz}$

$\mathrm{CDCl} 3$

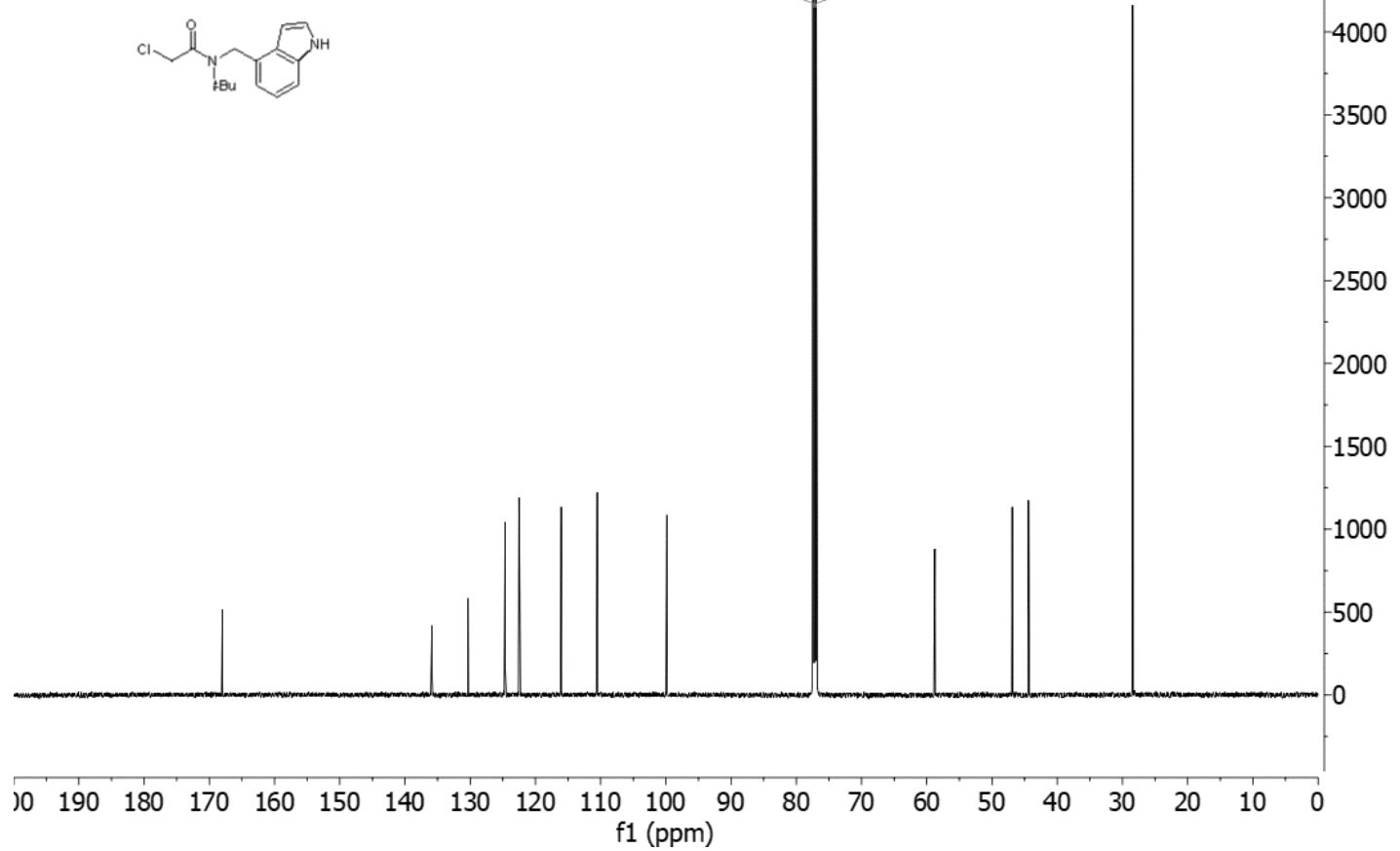




\section{${ }^{1} \mathrm{H}$ NMR of $\mathbf{S 3}$}

$600.18 \mathrm{MHz}$

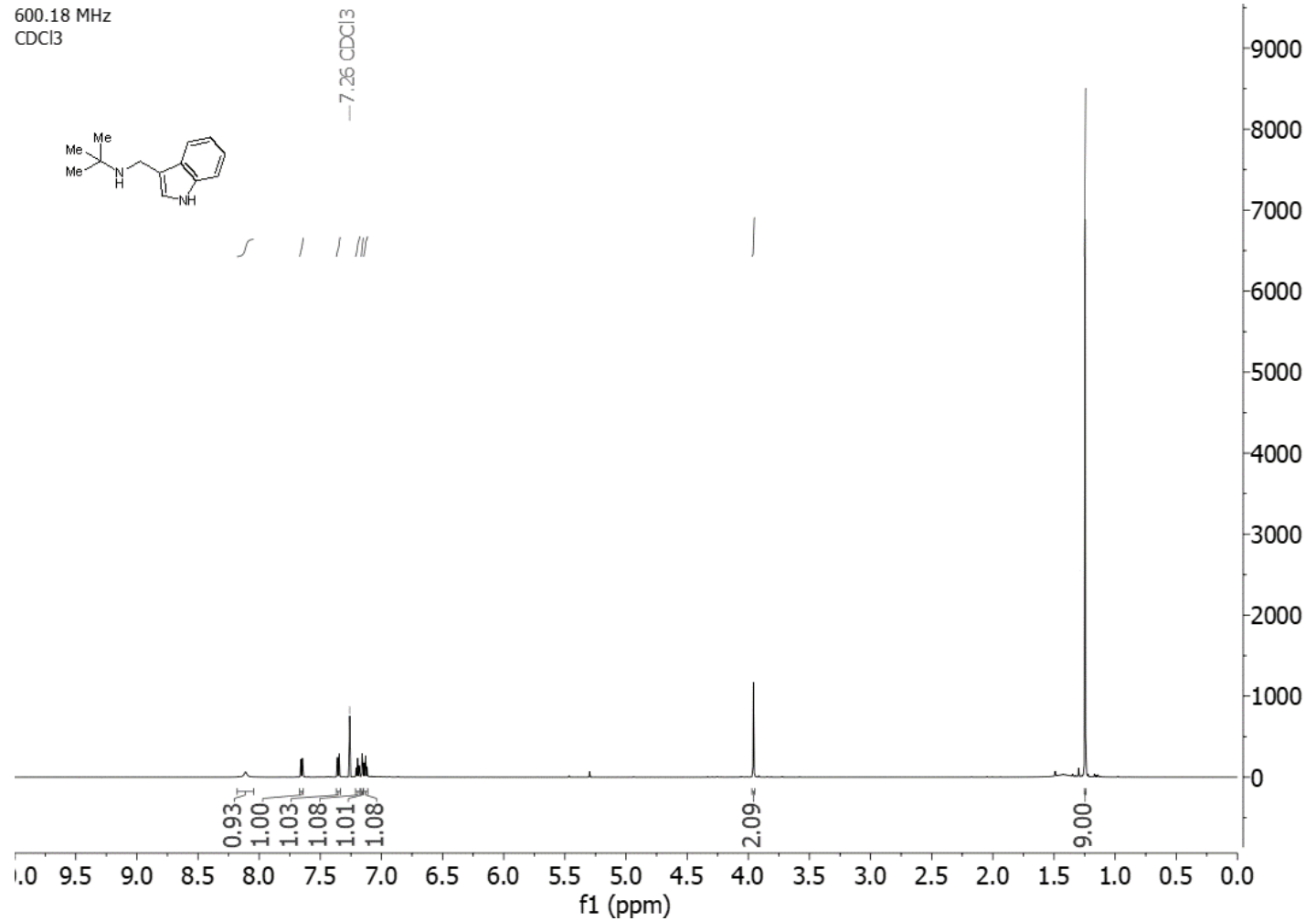

${ }^{13} \mathrm{C}$ NMR of $\mathbf{S 3}$

$150.93 \mathrm{MHz}$
$\mathrm{CDCl} 3$
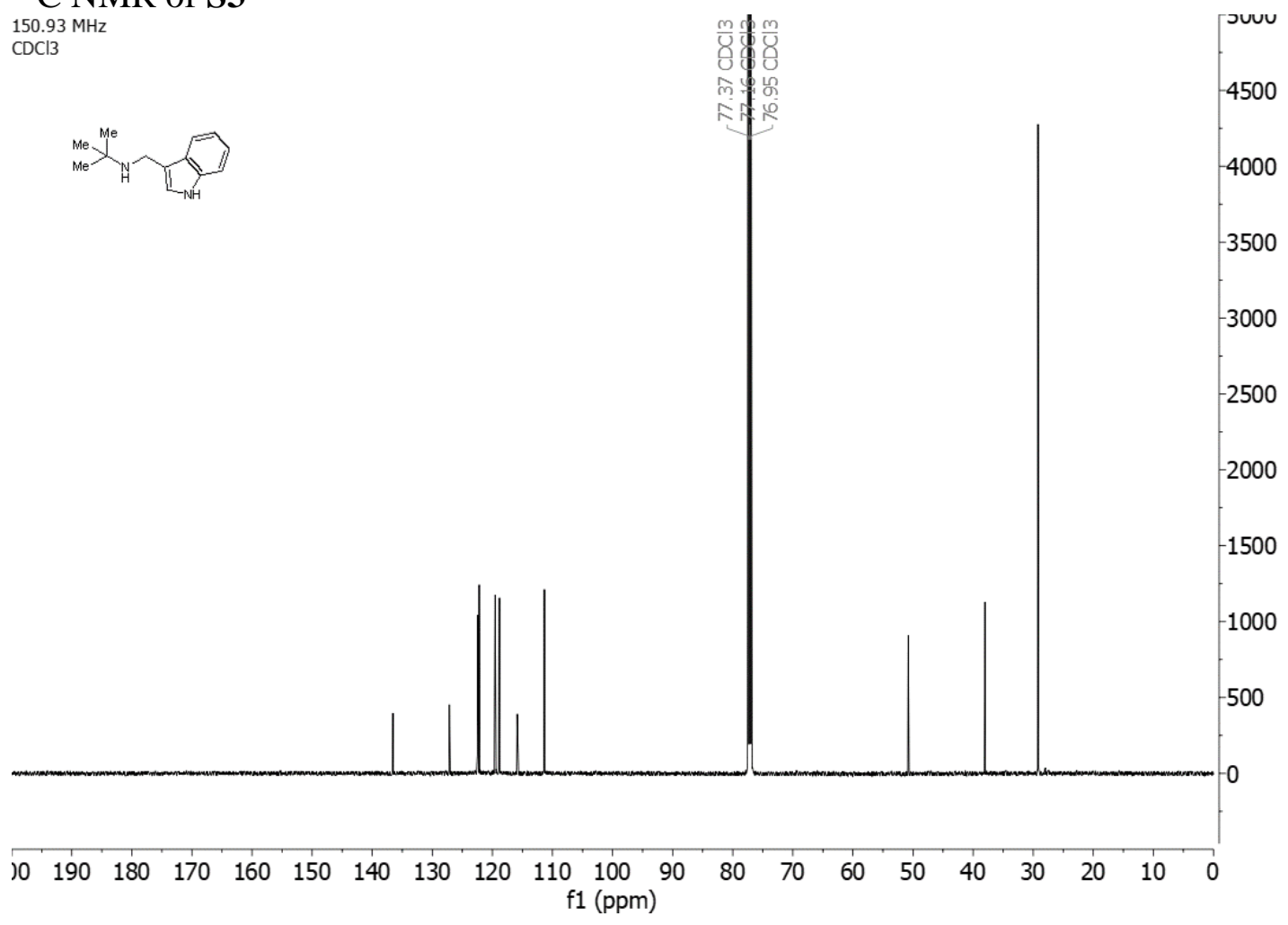
${ }^{1} \mathrm{H}$ NMR of $\mathbf{S 4}$

$150.93 \mathrm{MHz}$

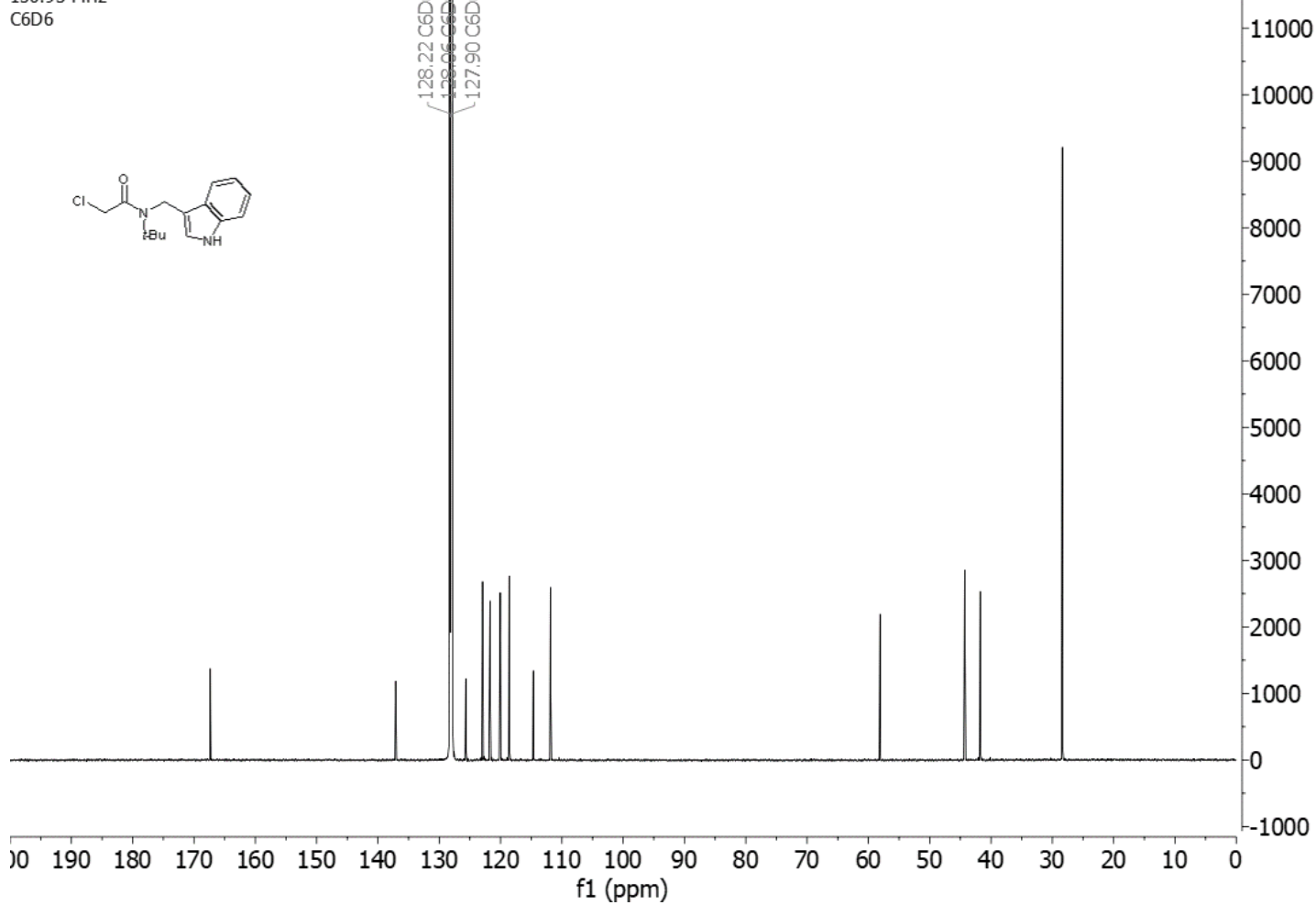

\section{${ }^{13} \mathrm{C}$ NMR of S4} $600.18 \mathrm{MHz}$
$\mathrm{C} 6 \mathrm{D} 6$<smiles>CN(Cc1cccc2ccccc12)C(=O)CCl</smiles>

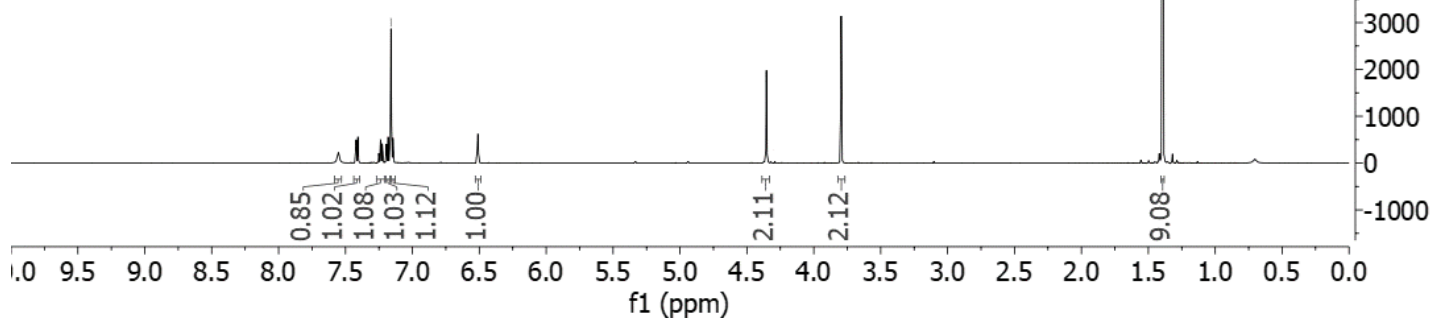


${ }^{1} \mathrm{H}$ NMR of $\mathbf{S 5}$

$600.18 \mathrm{MHz}$

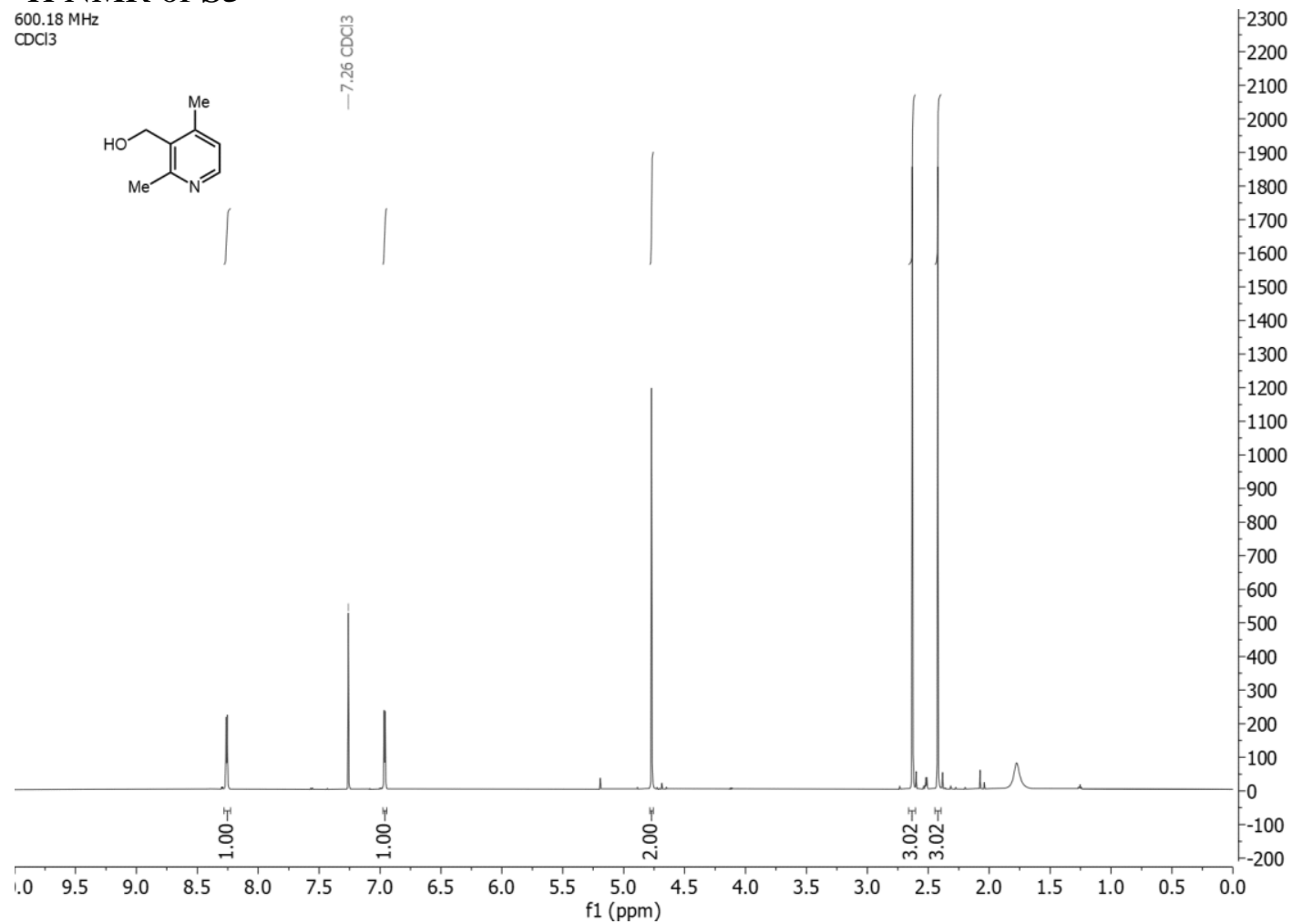

${ }^{13} \mathrm{C}$ NMR of $\mathbf{S 5}$ $150.93 \mathrm{MHz}$

$\mathrm{CDCl} 3$

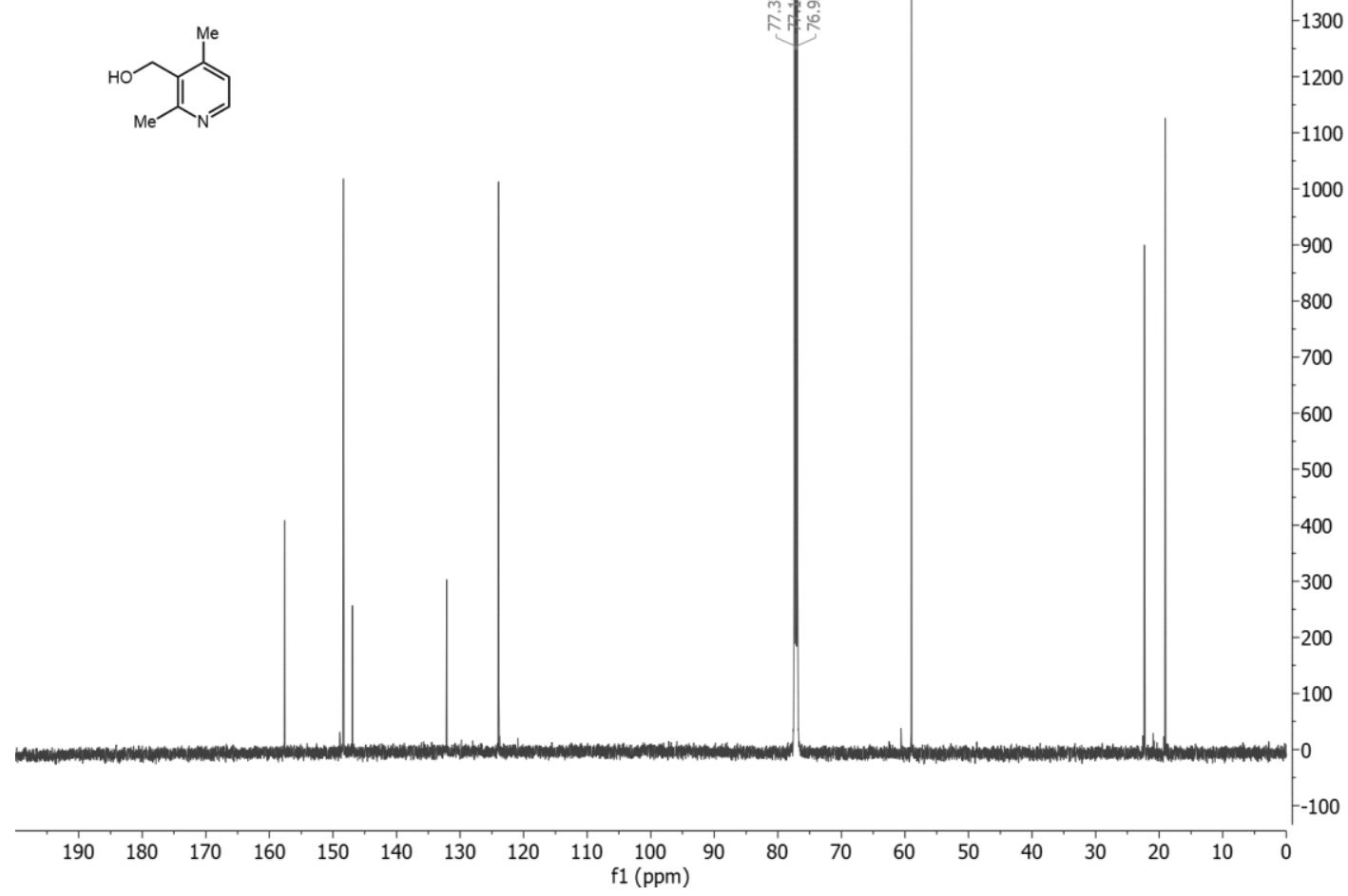


${ }^{1} \mathrm{H}$ NMR of S6

$600.18 \mathrm{MHz}$
$\mathrm{CDCl} 3$

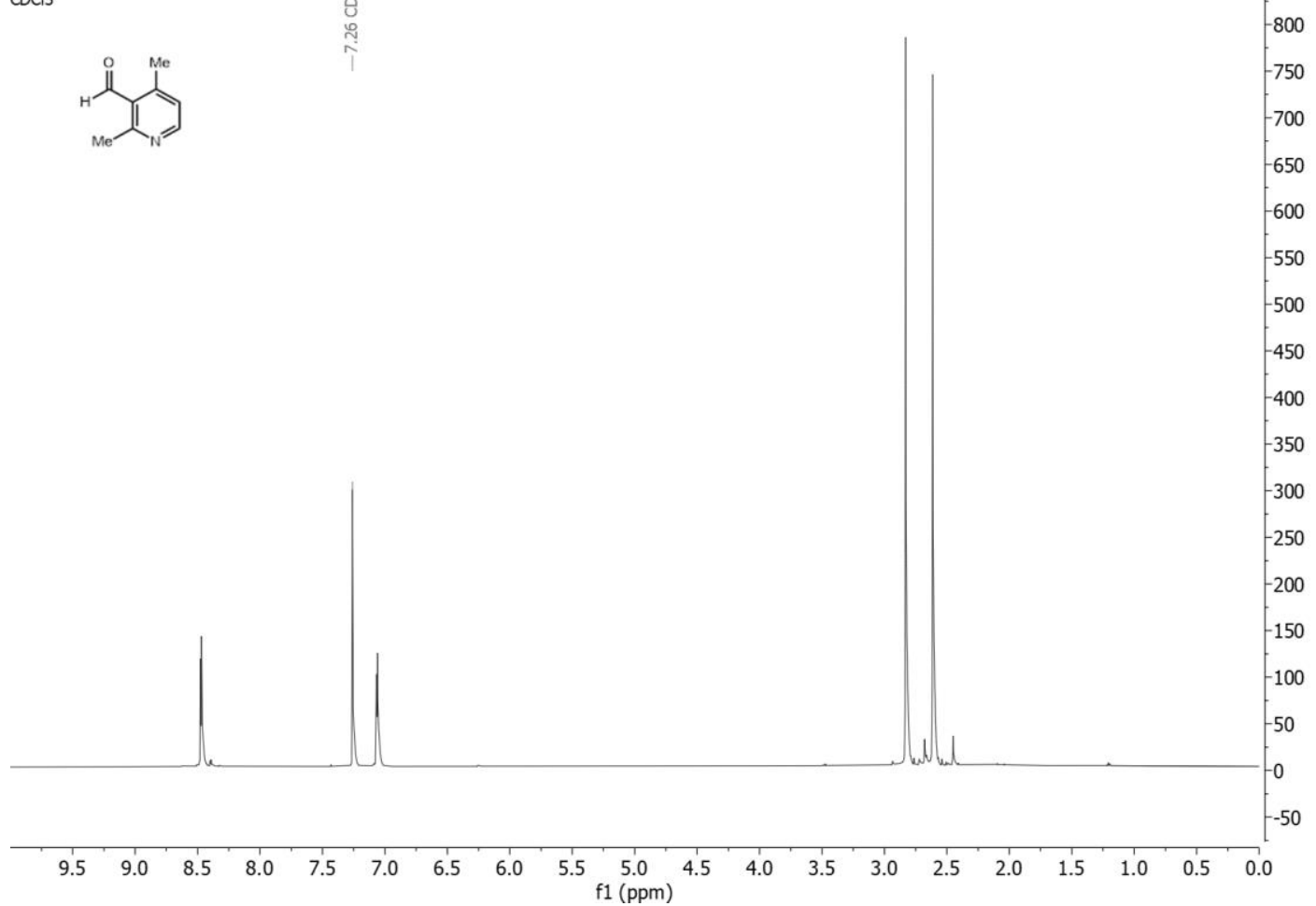

${ }^{13} \mathrm{C}$ NMR of S6

$150.93 \mathrm{MHz}$
$\mathrm{CDCl} 3$

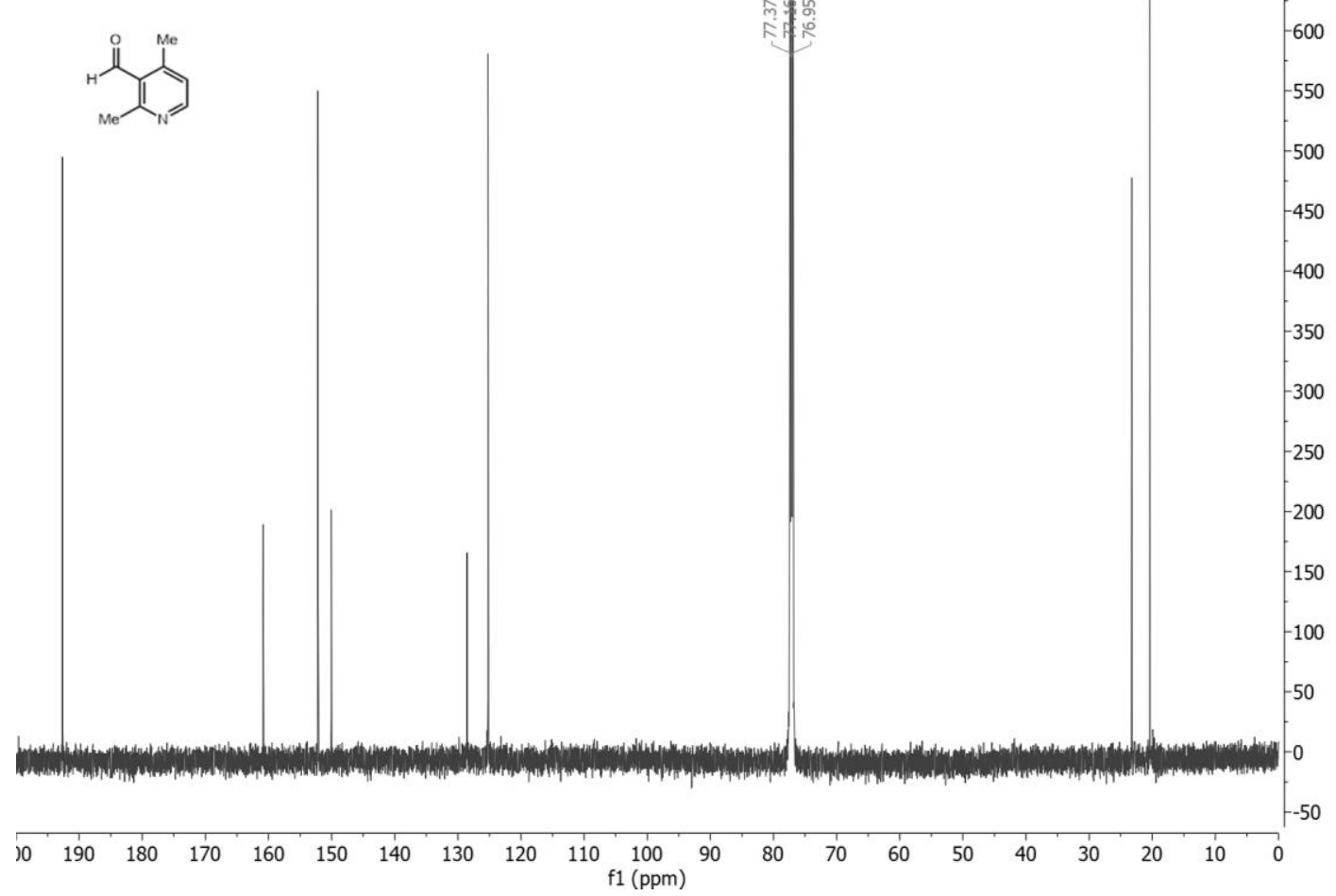




\section{${ }^{1} \mathrm{H}$ NMR of $\mathbf{S 7}$}

$600.18 \mathrm{MHz}$
$\mathrm{CDCl} 3 \mathrm{H} 1$<smiles>Cc1ccnc(C)c1CNC(C)(C)C</smiles>
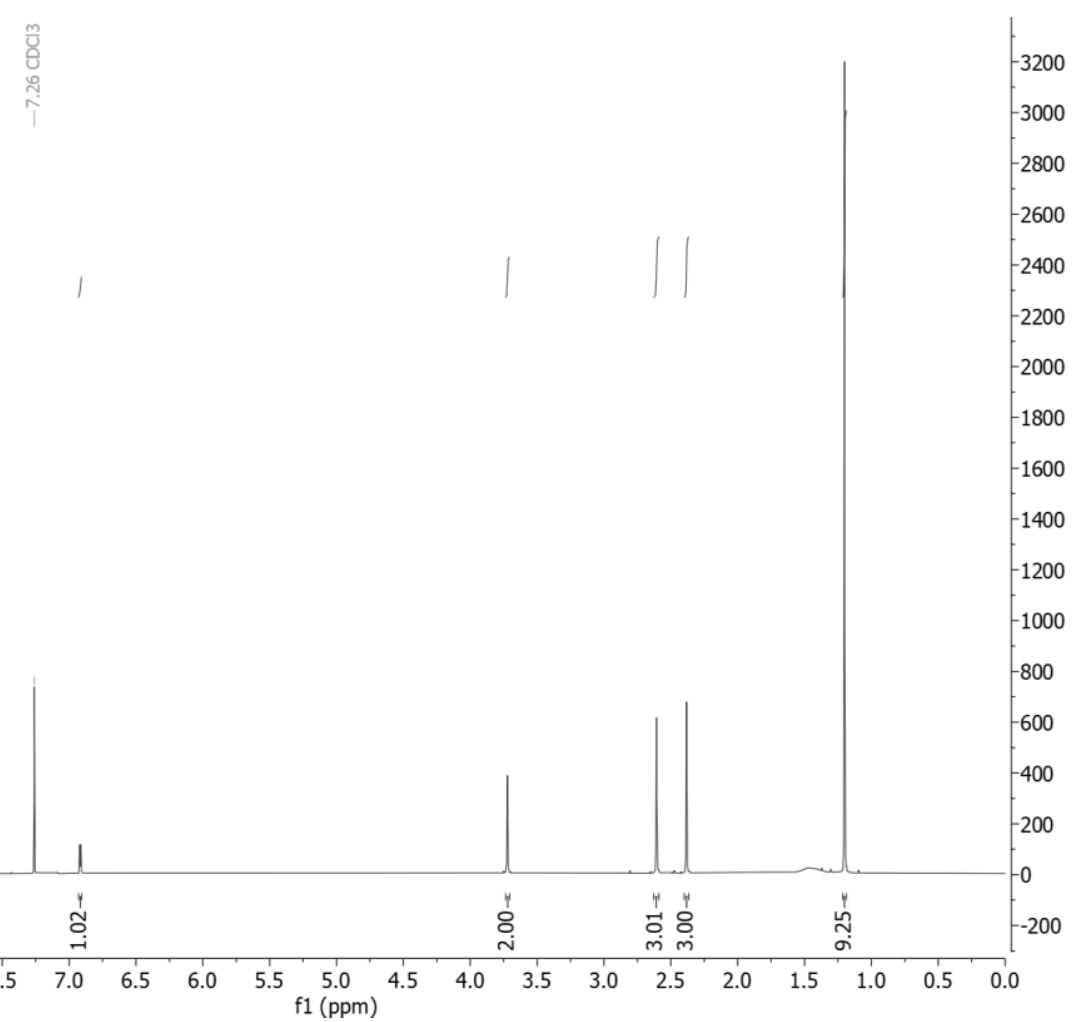

\section{${ }^{13} \mathrm{C}$ NMR of $\mathbf{S 7}$} $150.93 \mathrm{MHz}$
$\mathrm{CDCl} 3 \mathrm{C} 13$
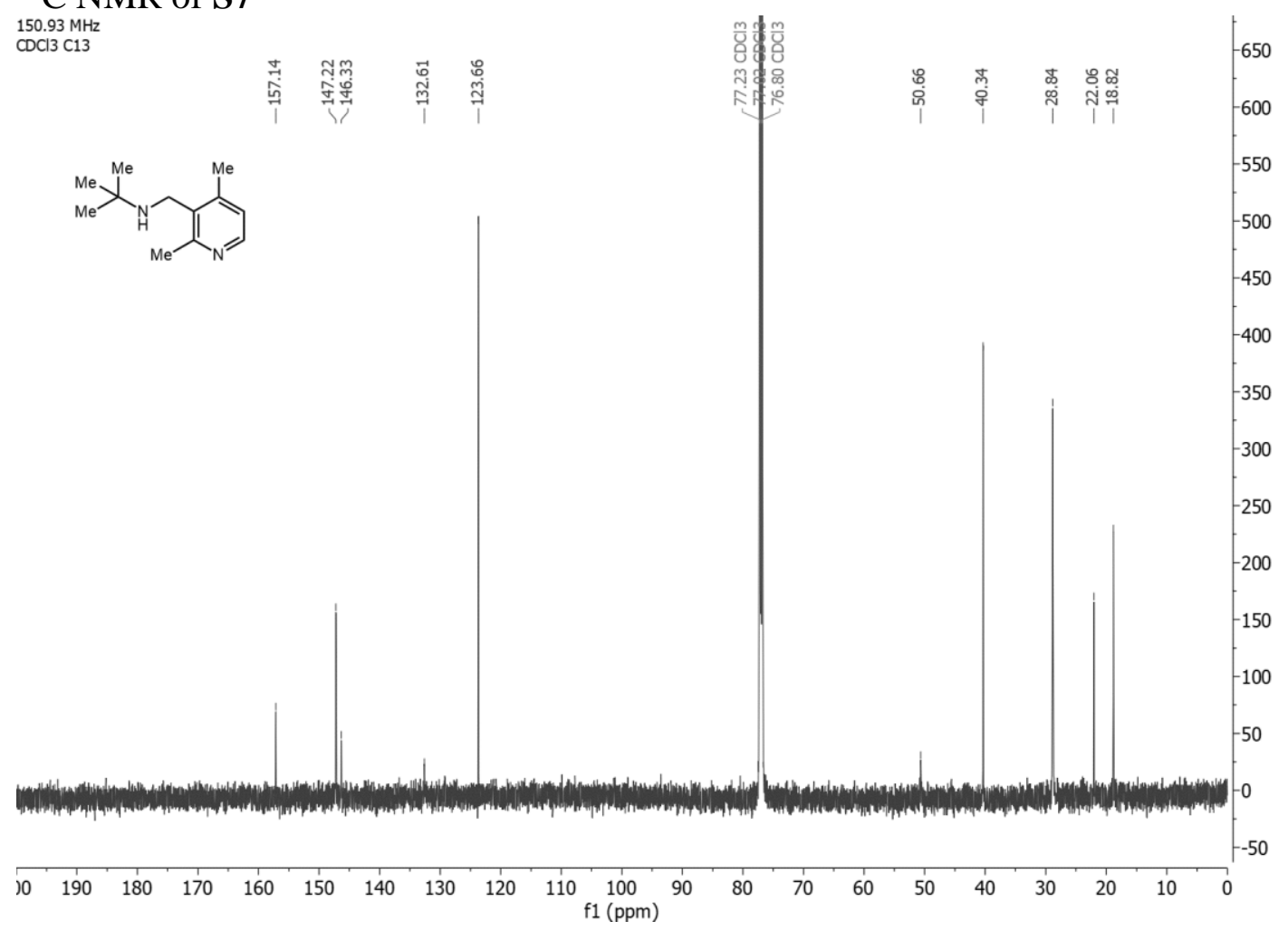
${ }^{1} \mathrm{H}$ NMR of $\mathbf{S 8}$

$600.18 \mathrm{MHz}$

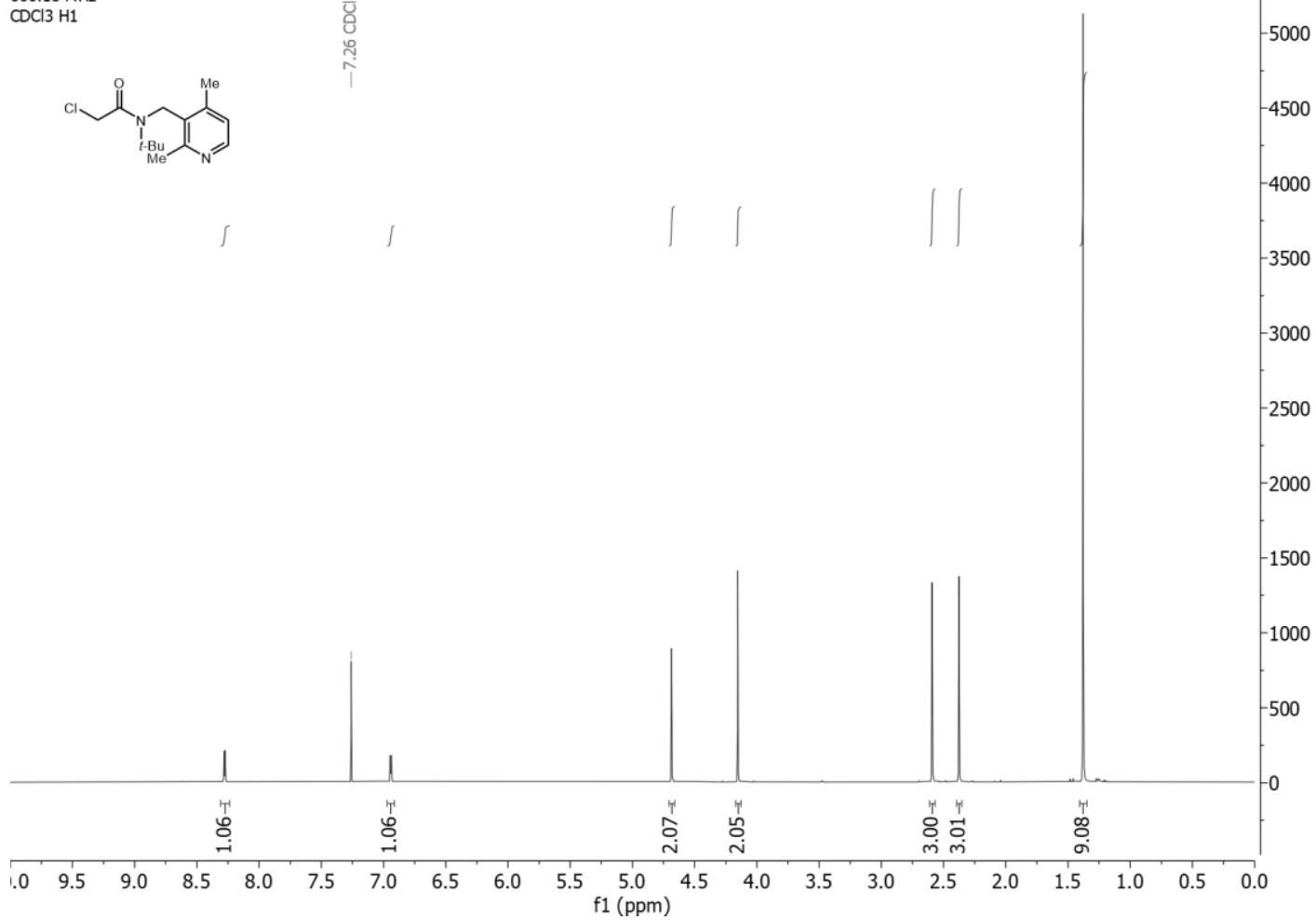

${ }^{13} \mathrm{C}$ NMR of $\mathbf{S 8}$

$150.93 \mathrm{MHz}$

CDCl3 C13

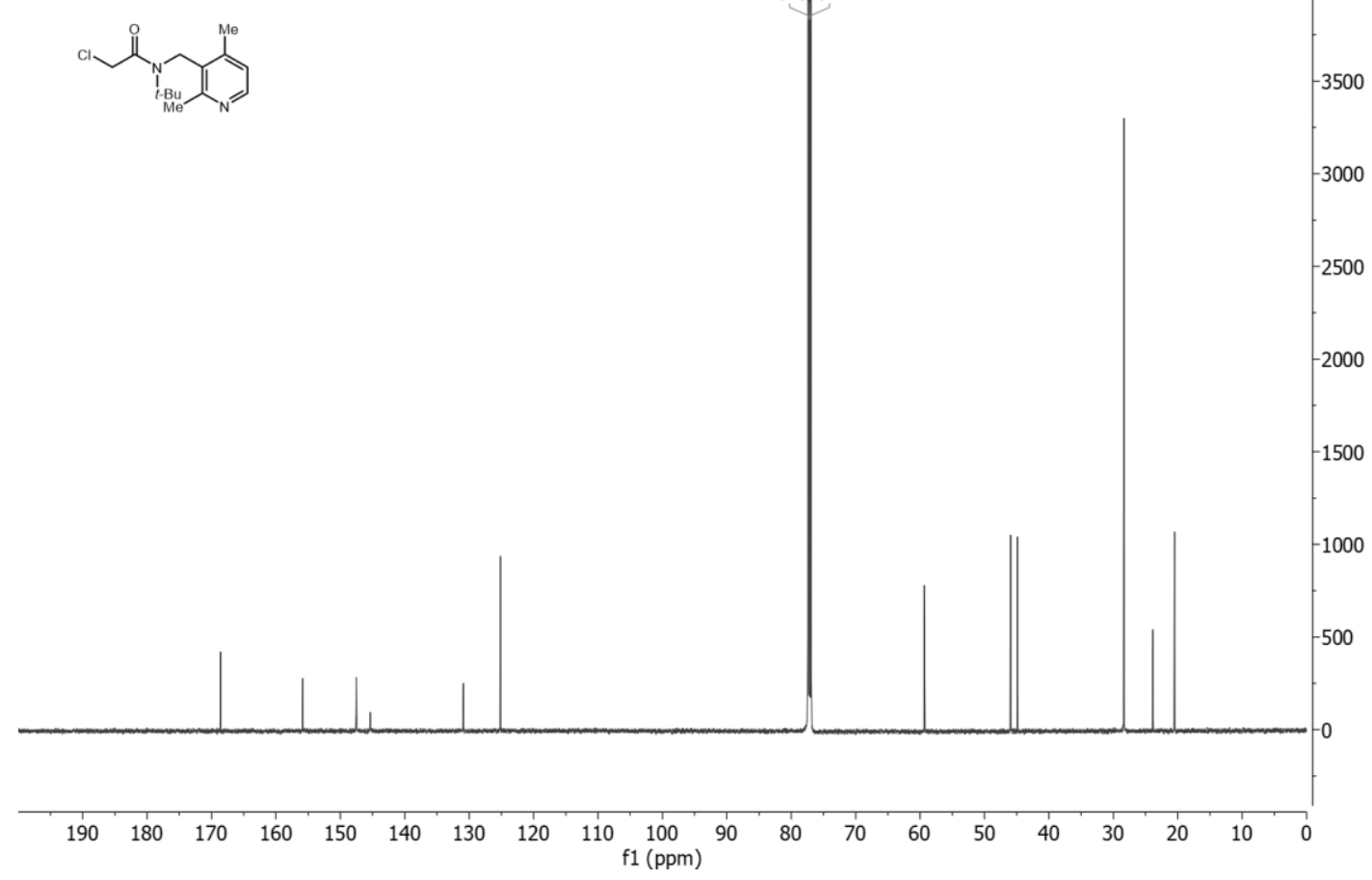




\section{${ }^{1}$ H NMR of S9}

$600.18 \mathrm{MHz}$
$\mathrm{CDCl} 1 \mathrm{HH}$

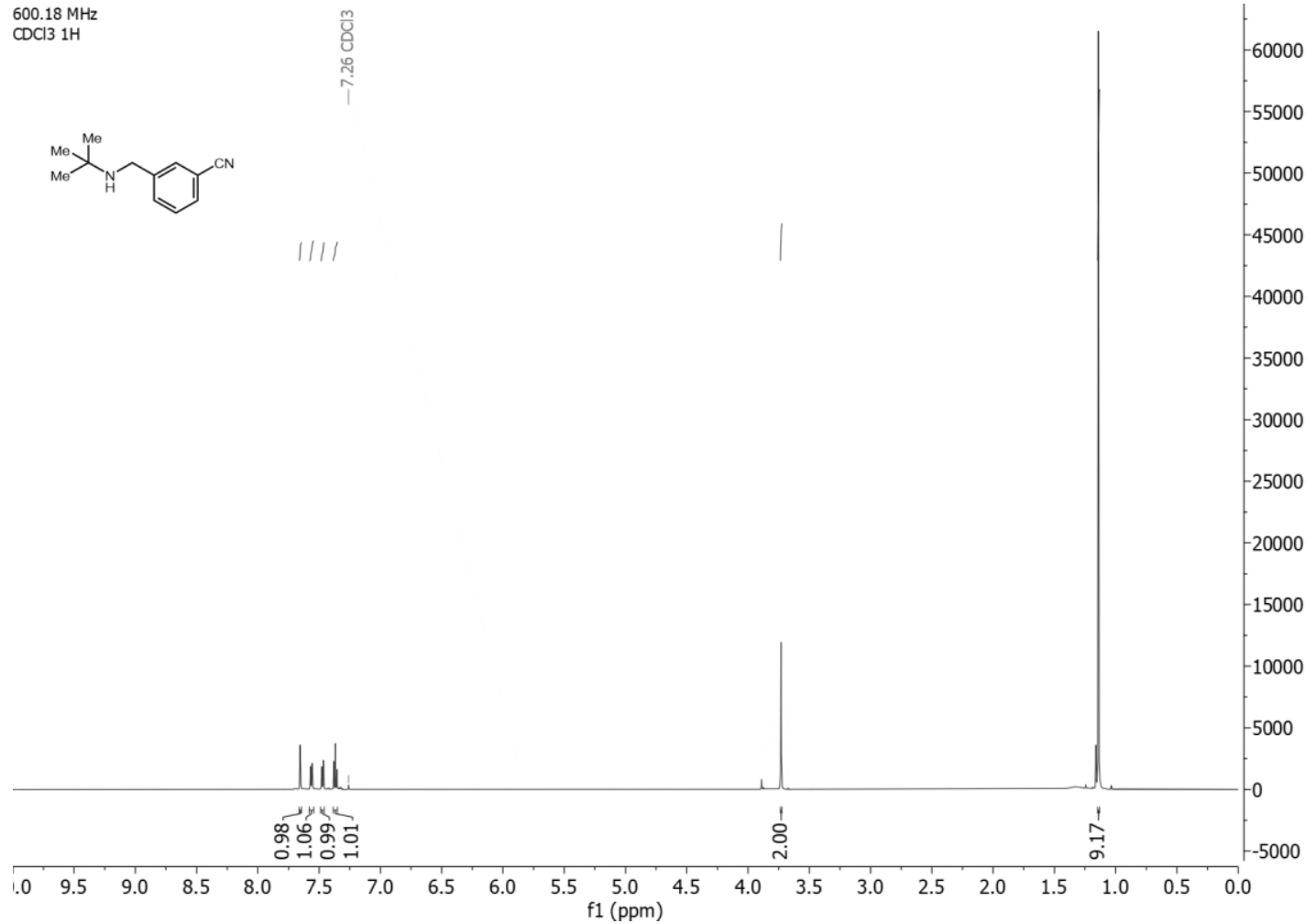

${ }^{13} \mathrm{C}$ NMR of $\mathbf{S 9}$

$150.93 \mathrm{MHz}$
$\mathrm{CDCl} 3 \mathrm{13C}$

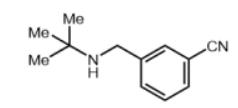

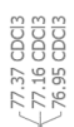

年 
${ }^{1} \mathrm{H}$ NMR of S10

${ }_{600.18 \mathrm{MHz}}^{6013 \mathrm{H} 1}$

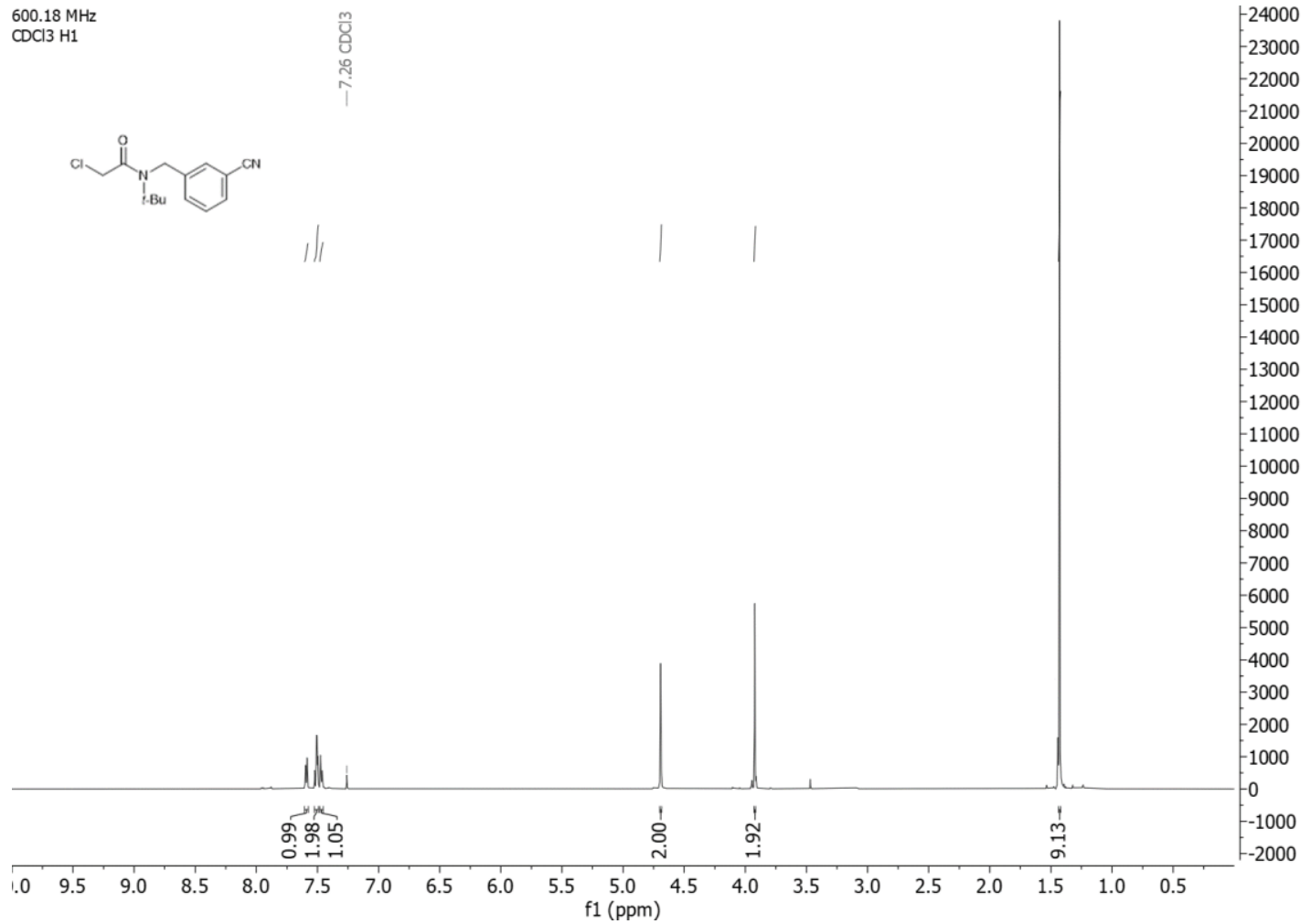

\section{${ }^{13} \mathrm{C}$ NMR of $\mathbf{S 1 0}$}

$150.93 \mathrm{MHz}$
$\mathrm{CDCl} 3 \mathrm{C} 13$

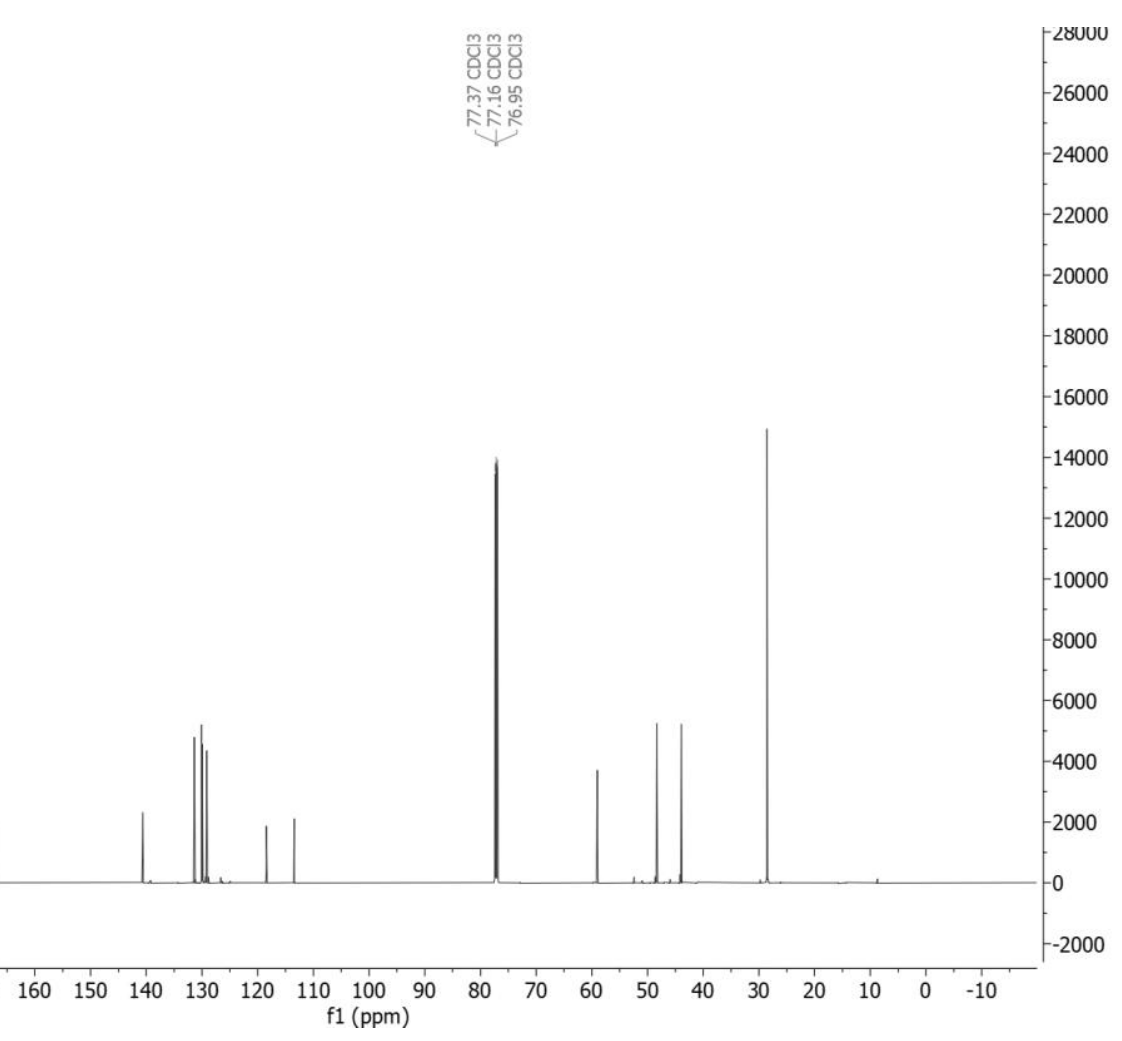

$\begin{array}{llllllllllll}20 & 210 & 200 & 190 & 180 & 170 & 160 & 150 & 140 & 130 & 120 & 110 \\ \mathrm{f} 1(\mathrm{ppm})\end{array}$ 

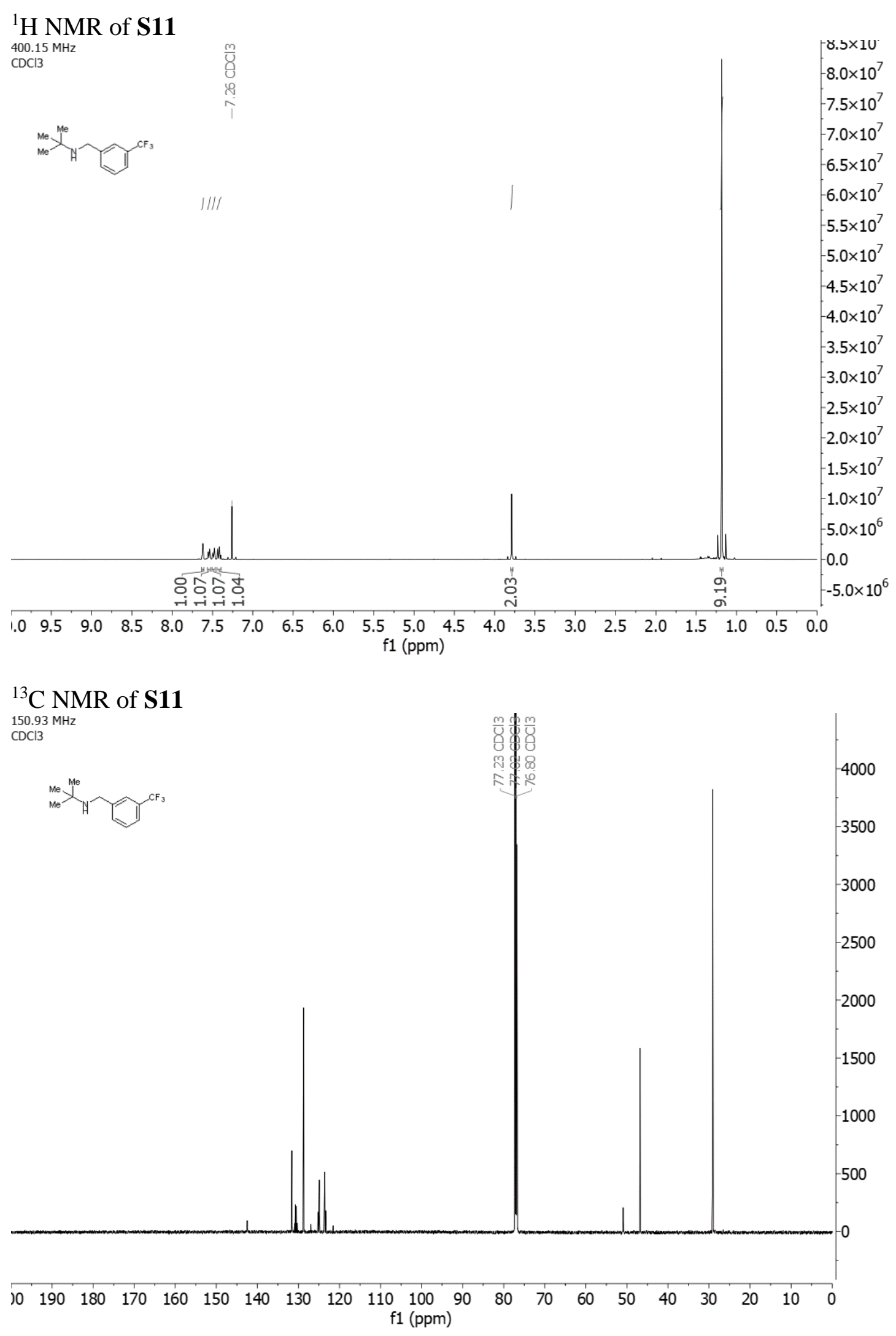
${ }^{1} \mathrm{H}$ NMR of $\mathbf{S 1 3}$

$400.15 \mathrm{MHz}$

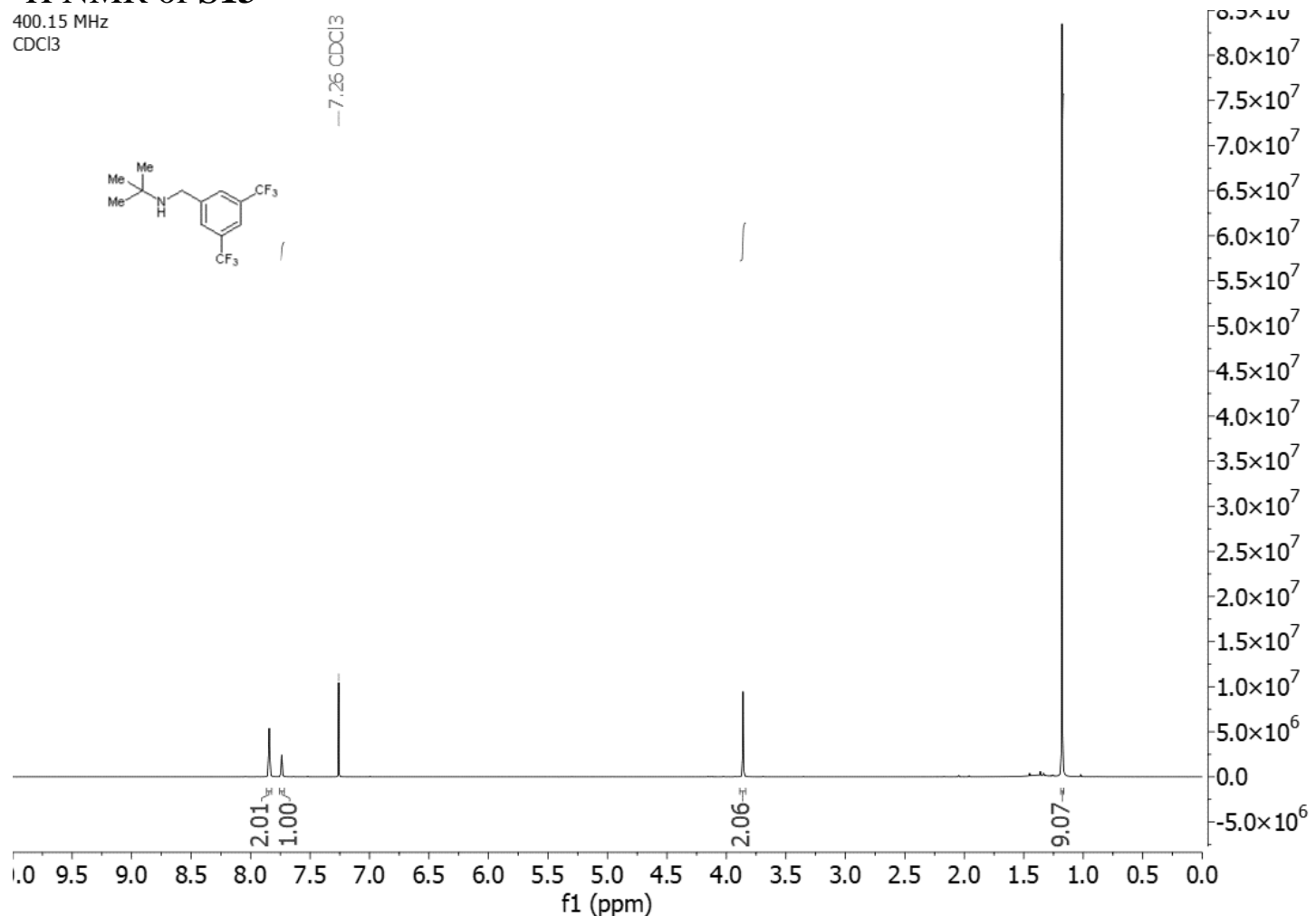

${ }^{13} \mathrm{C}$ NMR of $\mathbf{S 1 3}$

$150.93 \mathrm{MHz}$
$\mathrm{CDCl} 3$

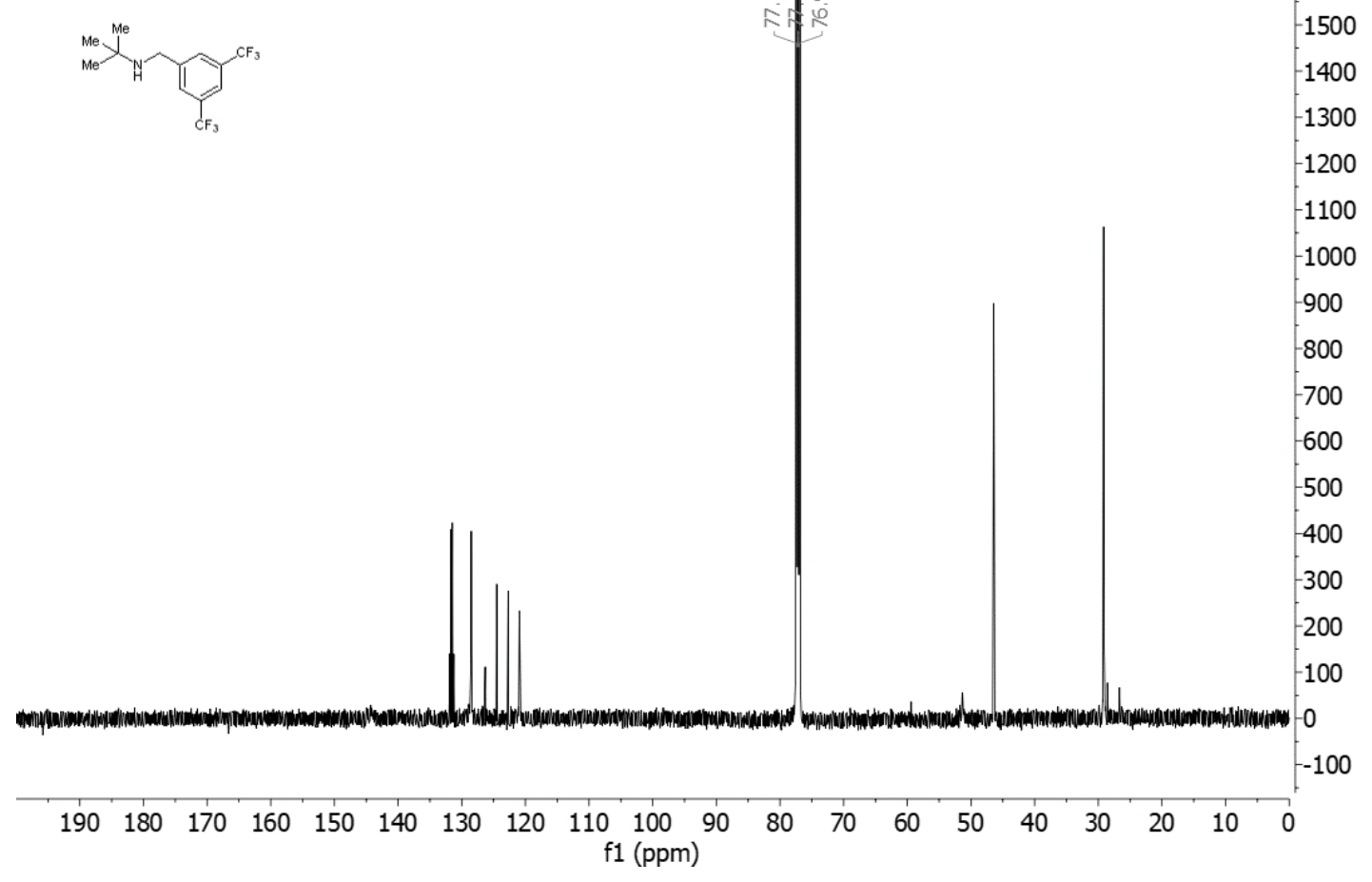



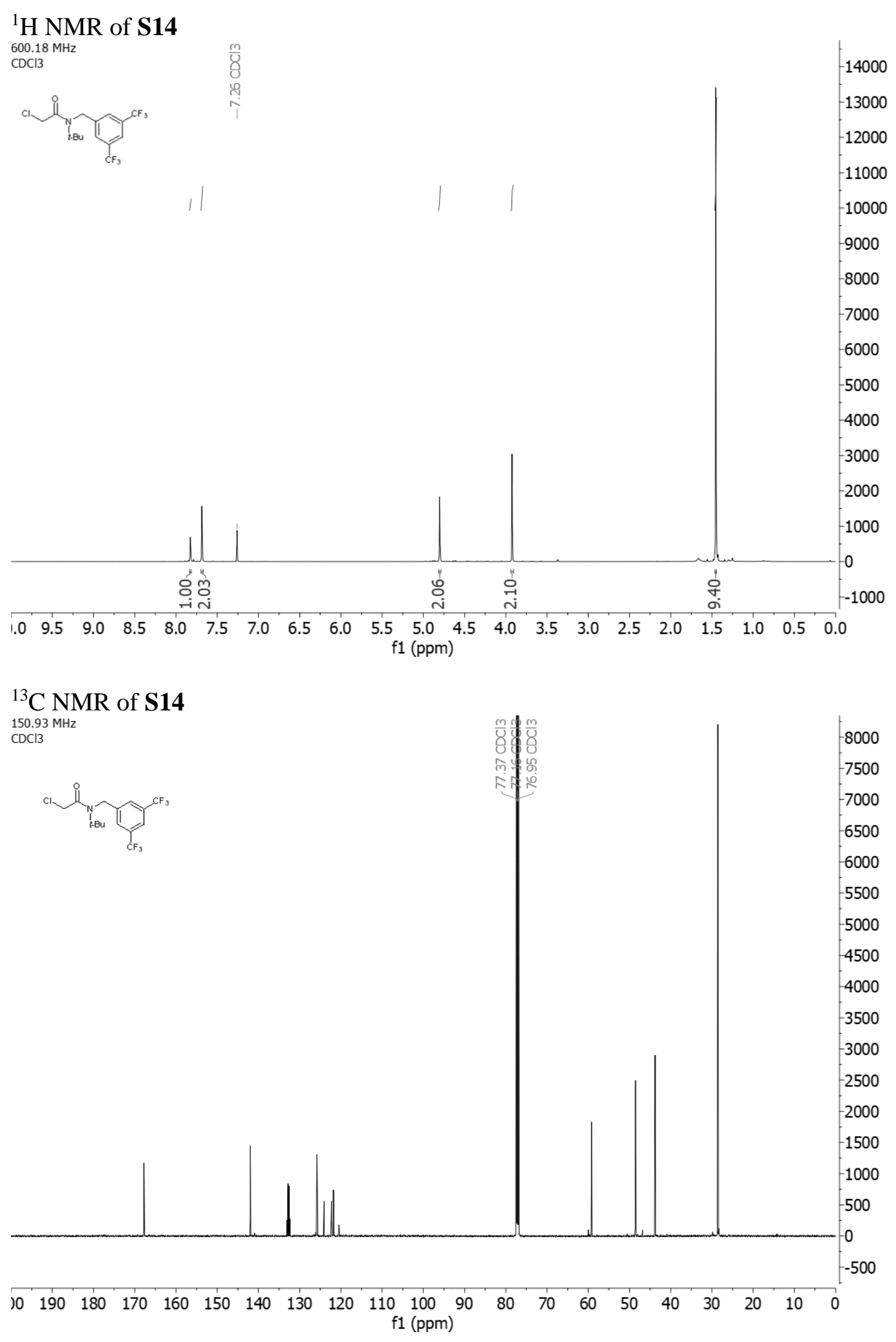
${ }^{1} \mathrm{H}$ NMR of $\mathbf{S 1 8}$

$600.18 \mathrm{MHz}$
$\mathrm{CDCl} 3 \mathrm{H} 1$

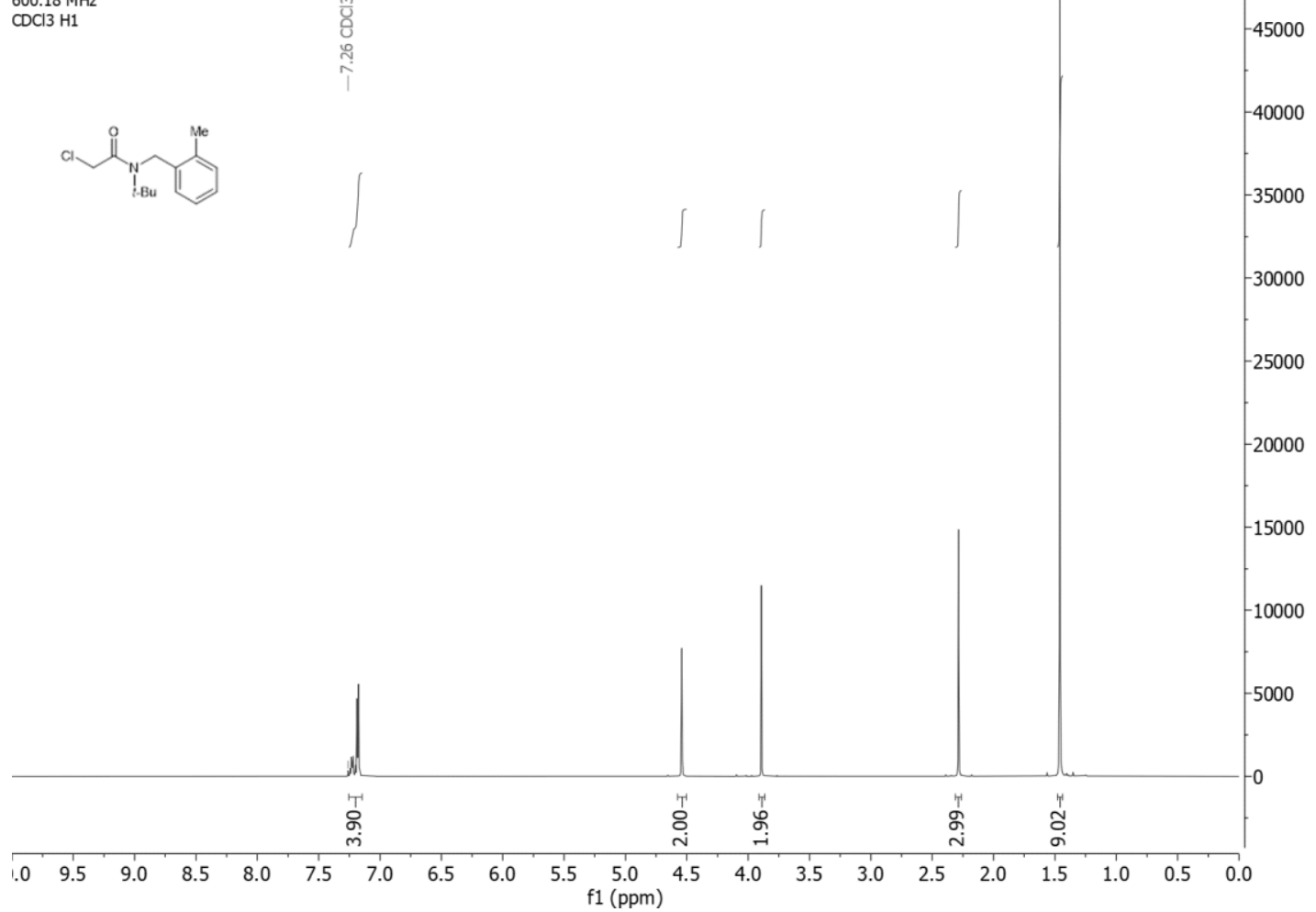

\section{${ }^{13} \mathrm{C}$ NMR of $\mathbf{S 1 8}$} $150.93 \mathrm{MHz}$
$\mathrm{CDCl} 3 \mathrm{C} 13$

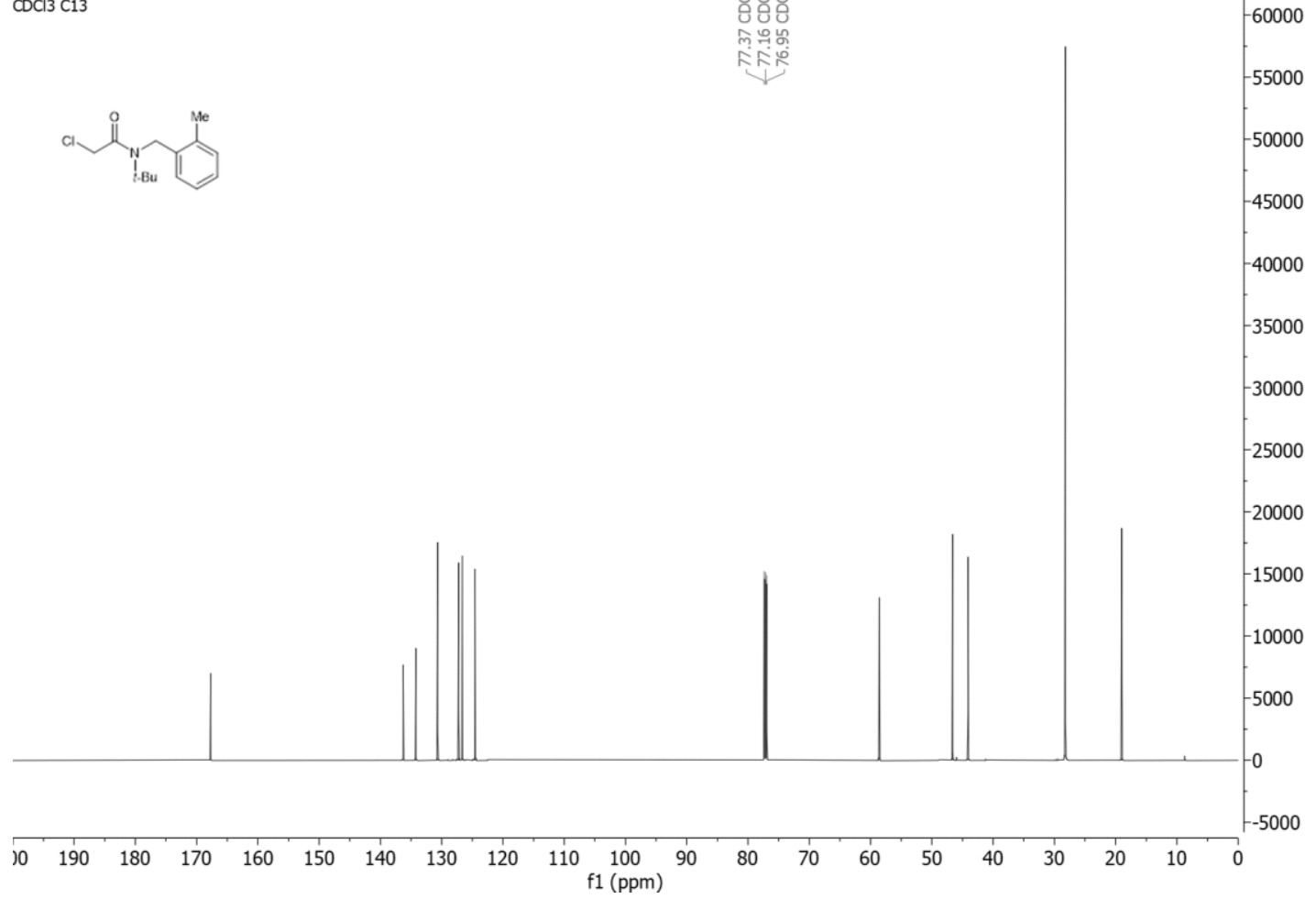


${ }^{1} \mathrm{H}$ NMR of $\mathbf{S 2 0}$

${ }_{600.18 \mathrm{MHz}}$

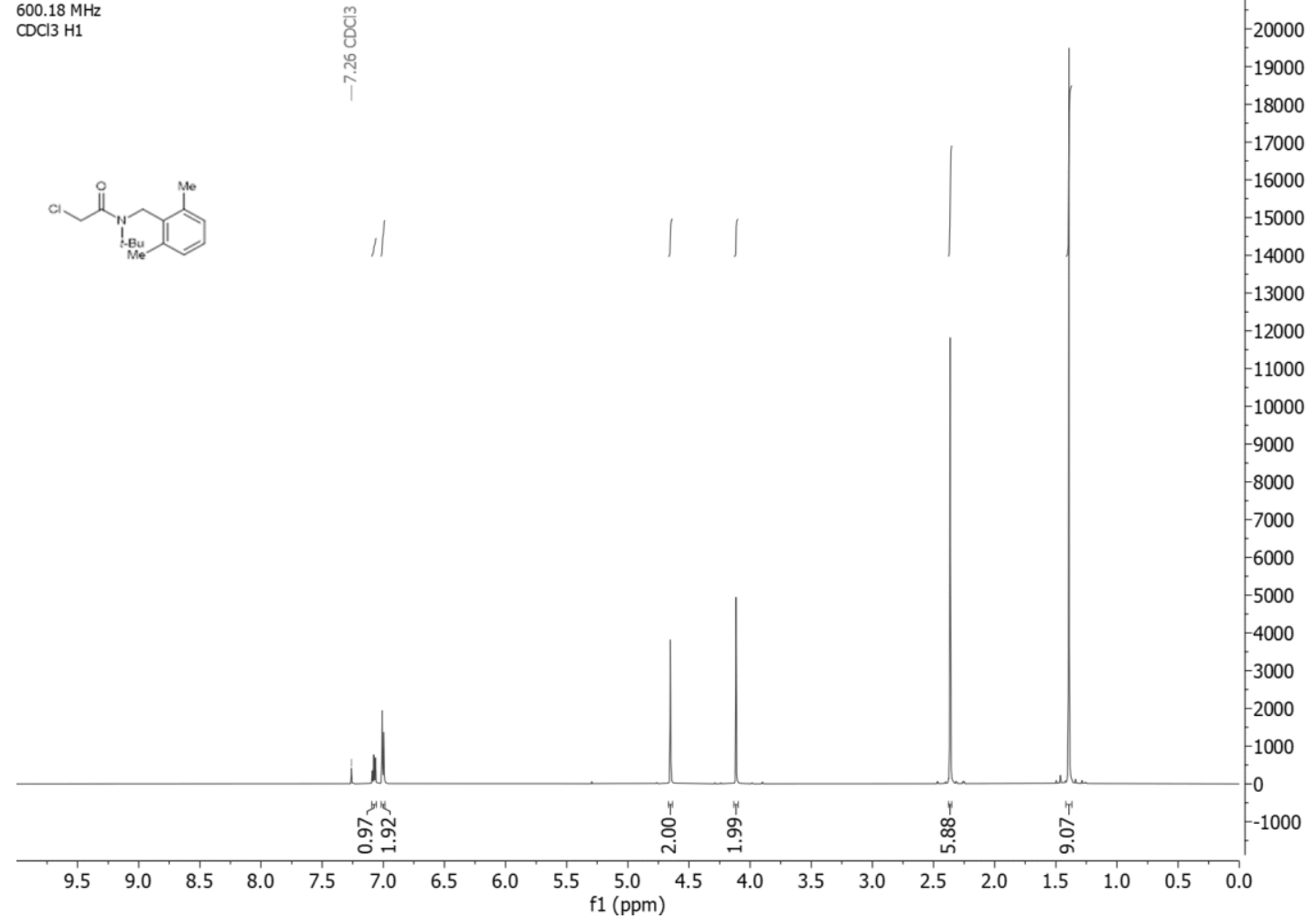

\section{${ }^{13} \mathrm{C}$ NMR of $\mathbf{S 2 0}$}

${ }_{150.93 \mathrm{MHz}}$

$\mathrm{CDCl} 3 \mathrm{C} 13$

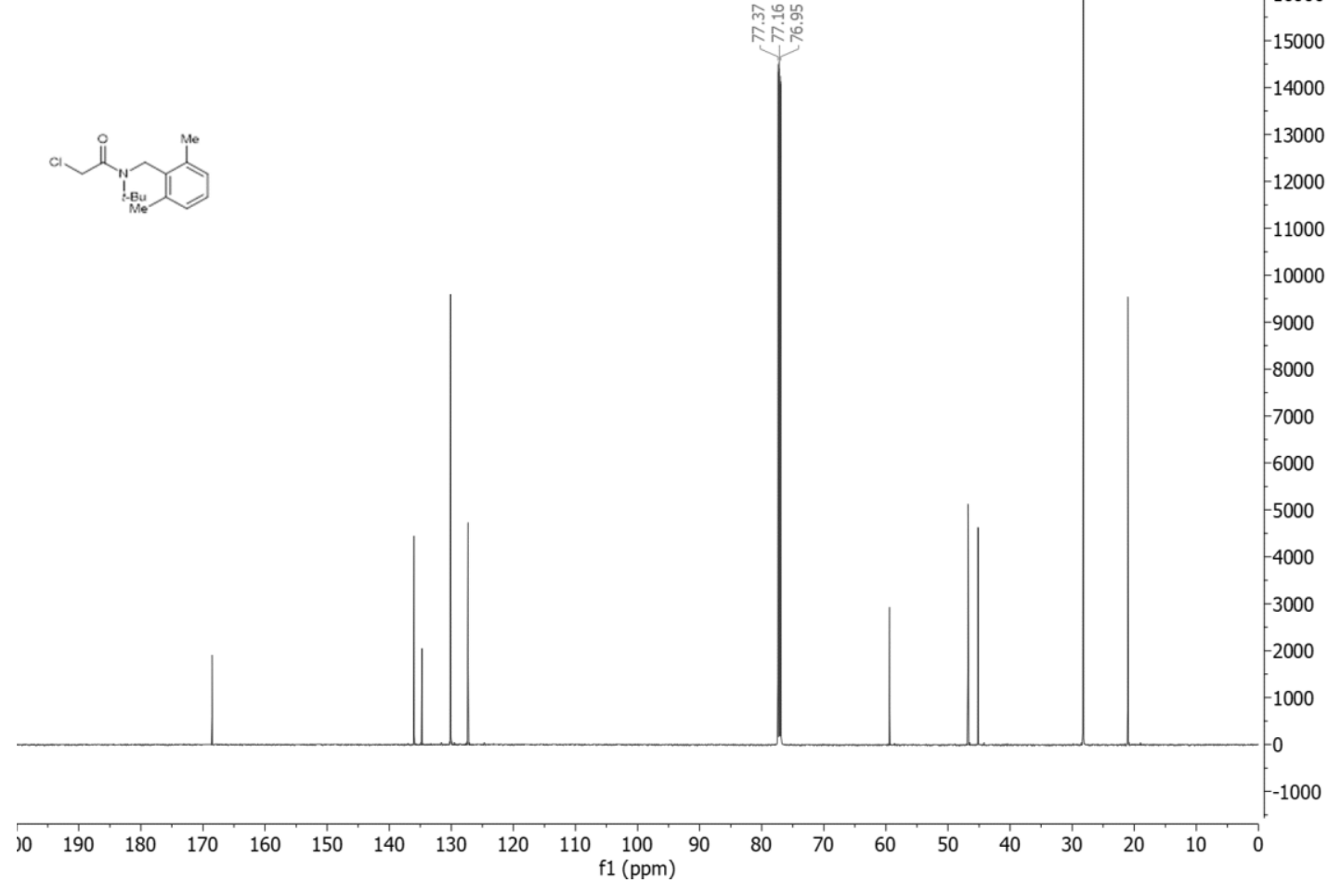


${ }^{1} \mathrm{H}$ NMR of $\mathbf{S 2 2}$

$600.18 \mathrm{MHz}$
$\mathrm{CDCl} 3 \mathrm{H} 1$

$\mathrm{Hz}$
0
0

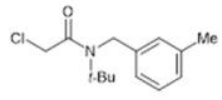

m
0
ñ
$i$

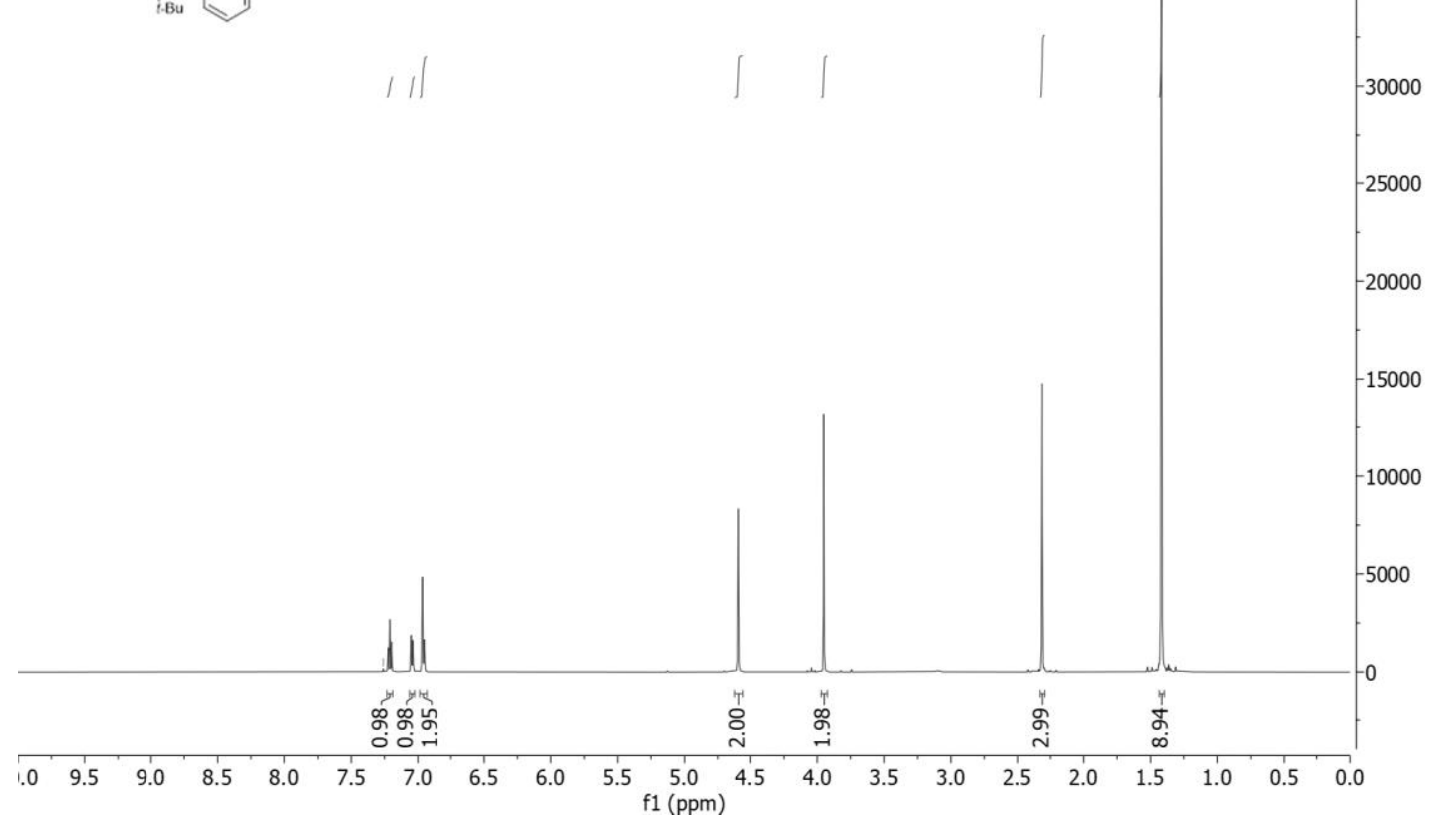

\section{${ }^{13} \mathrm{C}$ NMR of $\mathbf{S 2 2}$}

$150.93 \mathrm{MHz}$
$\mathrm{CDCl} 3 \mathrm{C} 13$

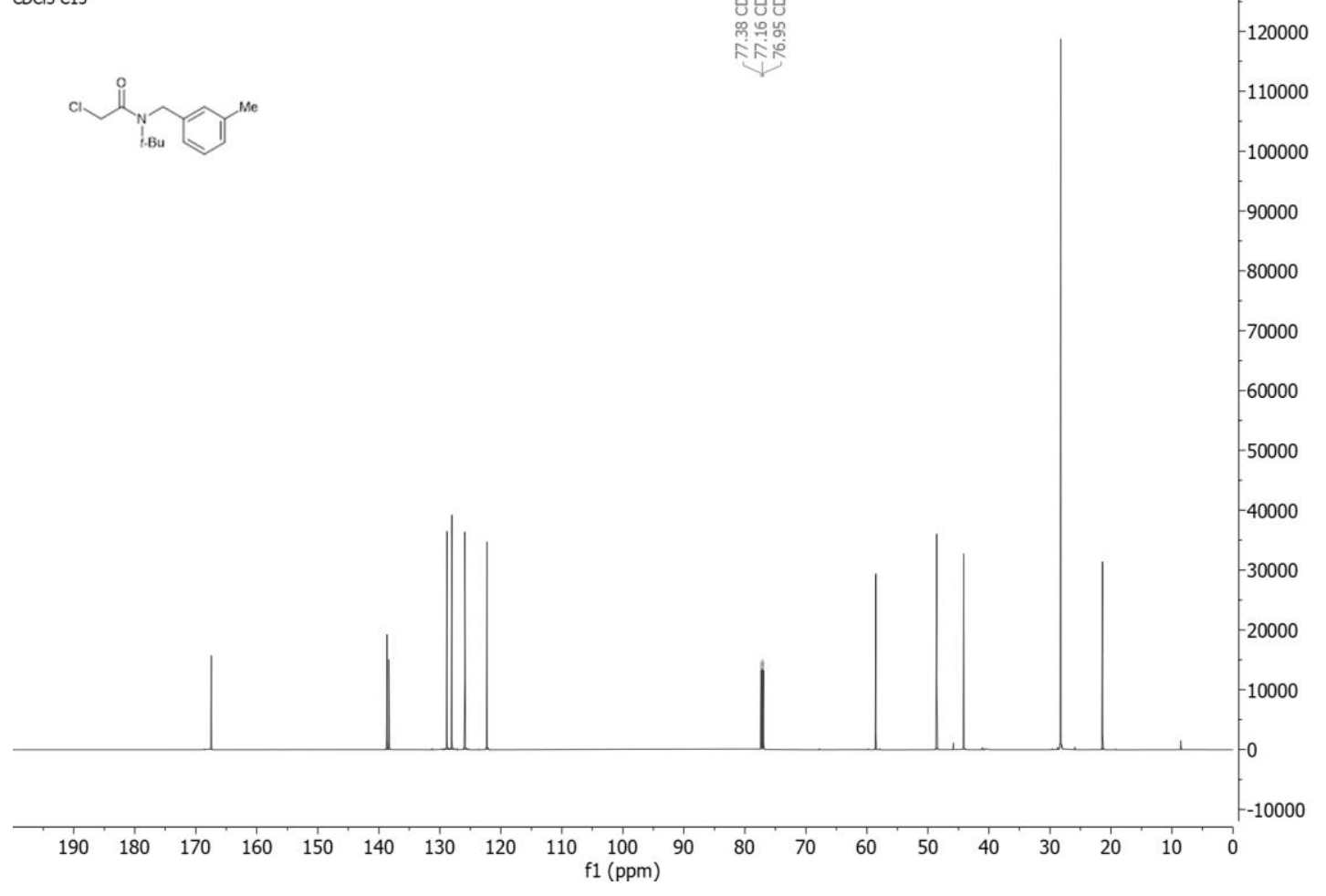


${ }^{1} \mathrm{H}$ NMR of $\mathbf{S 2 4}$

$600.18 \mathrm{MHz}$

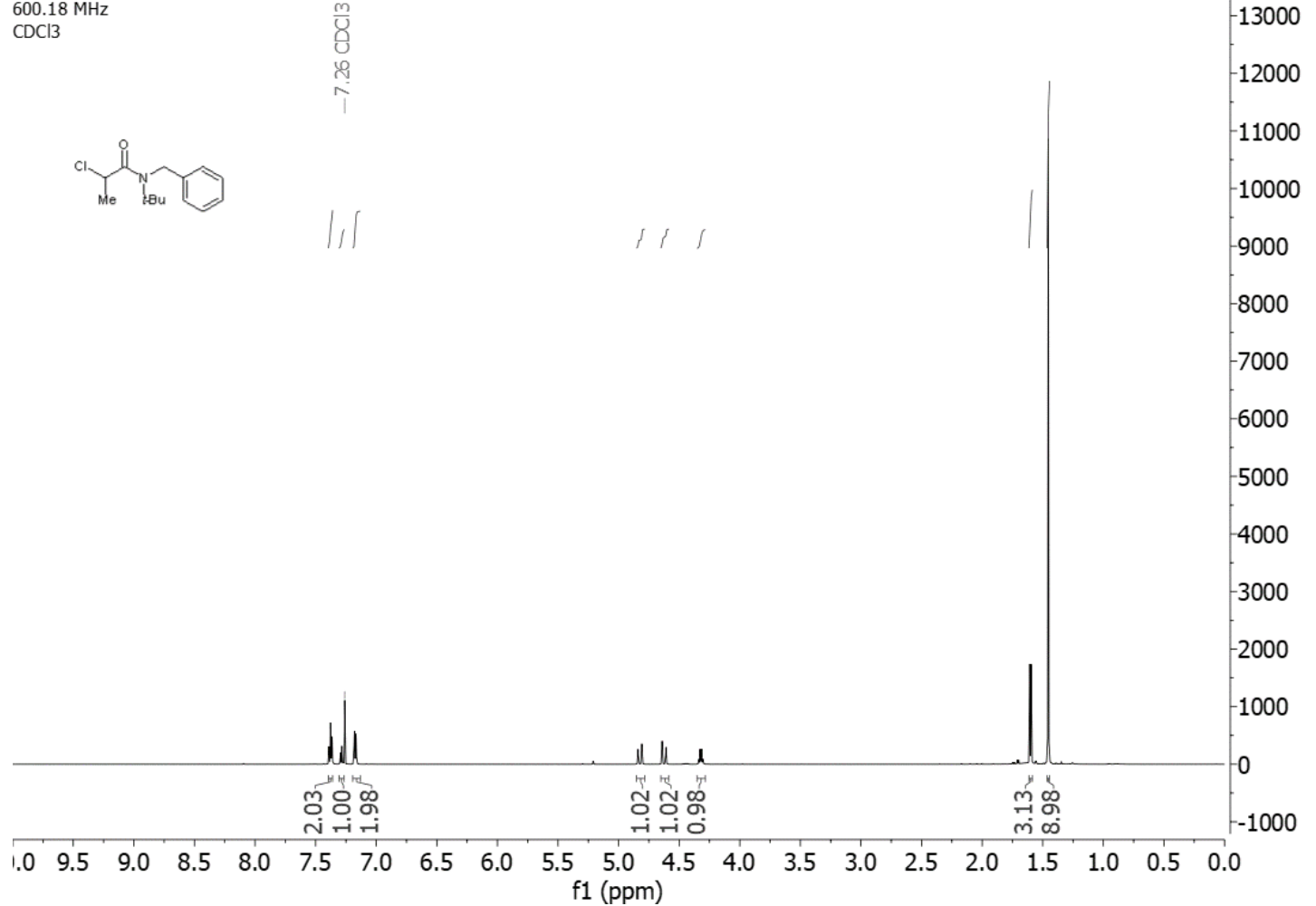

${ }^{13} \mathrm{C}$ NMR of $\mathbf{S 2 4}$

$150.93 \mathrm{MHz}$

$\mathrm{CDCl} 3$

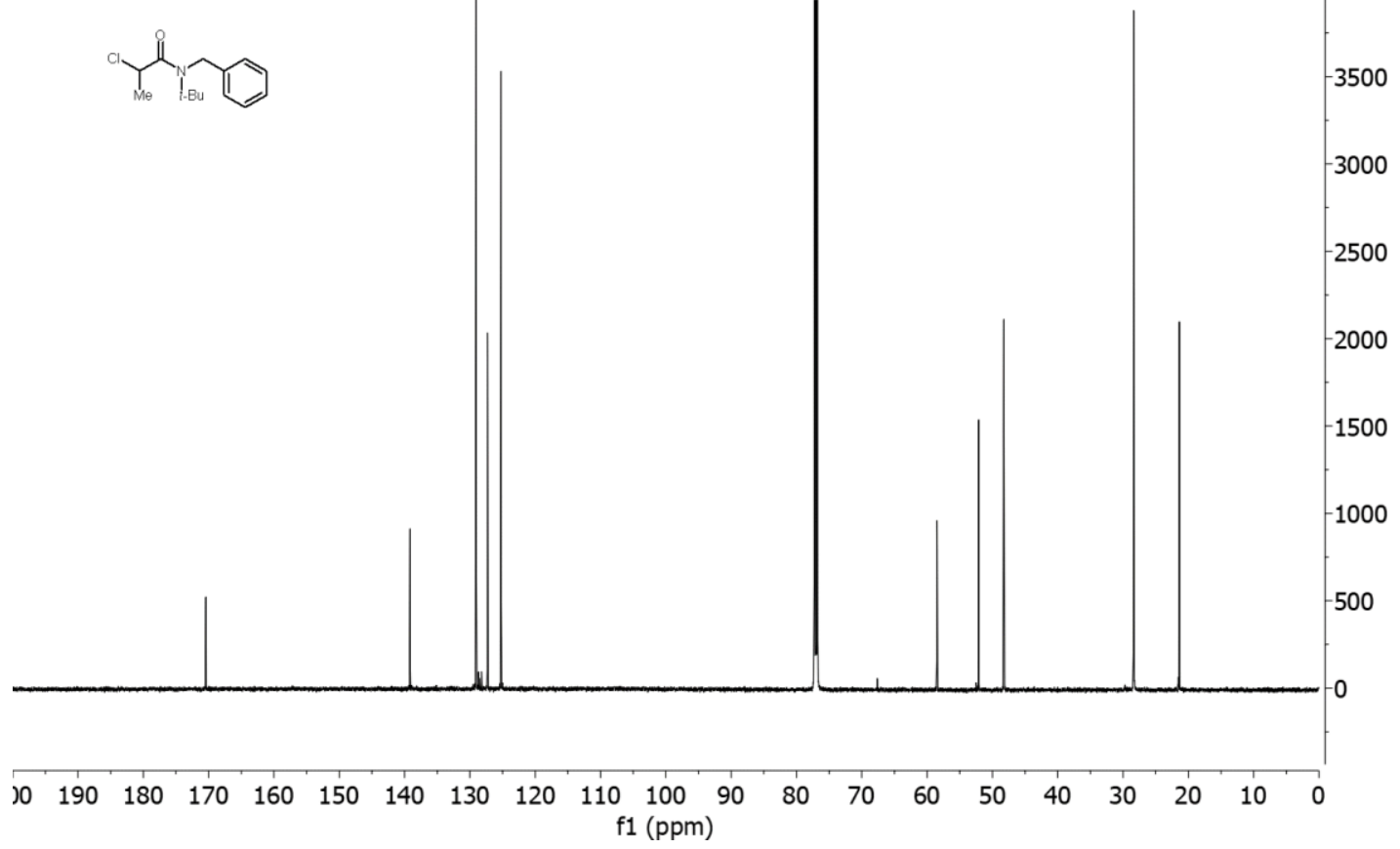


${ }^{1} \mathrm{H}$ NMR of $\mathbf{S 2 6}$

$500.29 \mathrm{MHz}$

cdcl3

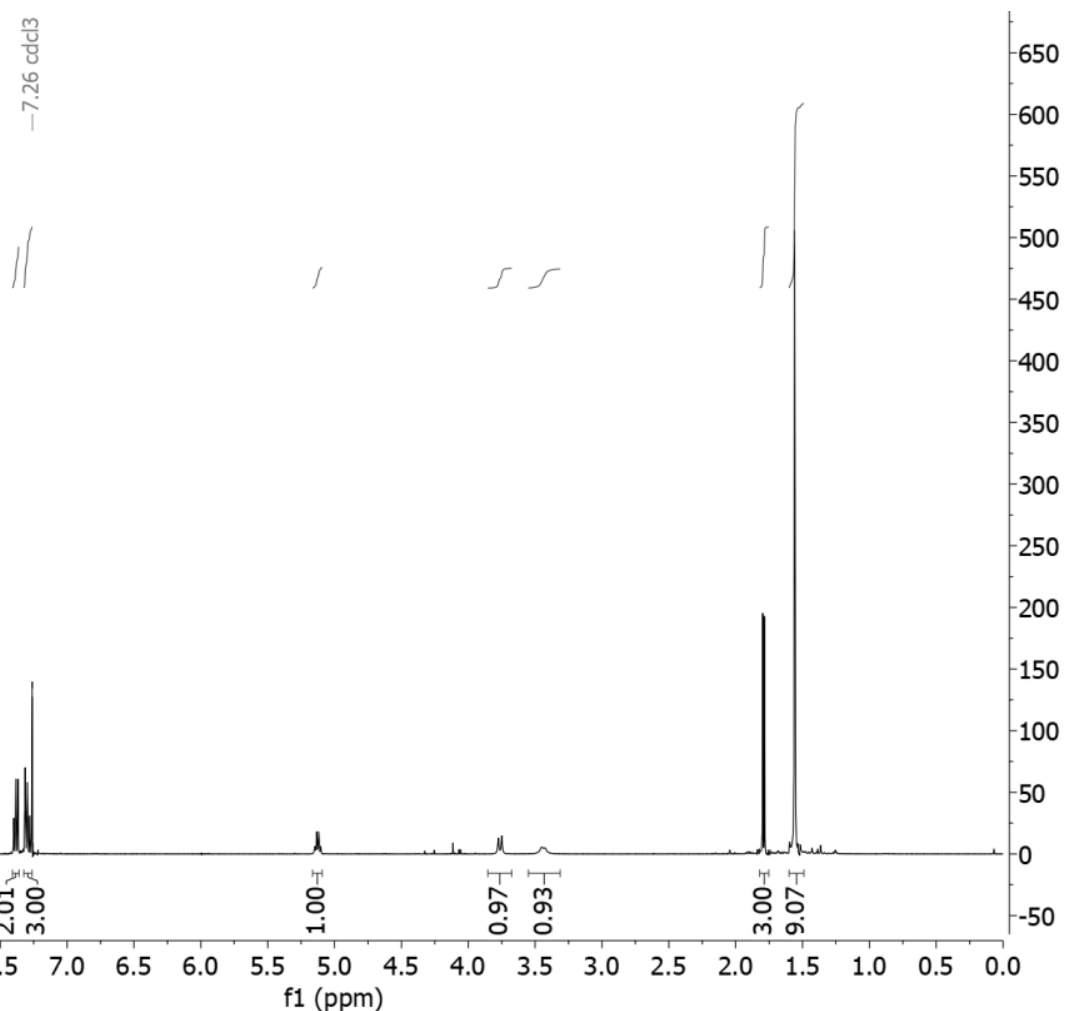

${ }^{13} \mathrm{C}$ NMR of $\mathbf{S 2 6}$

$100.54 \mathrm{MHz}$

$\mathrm{CDCl} 3$

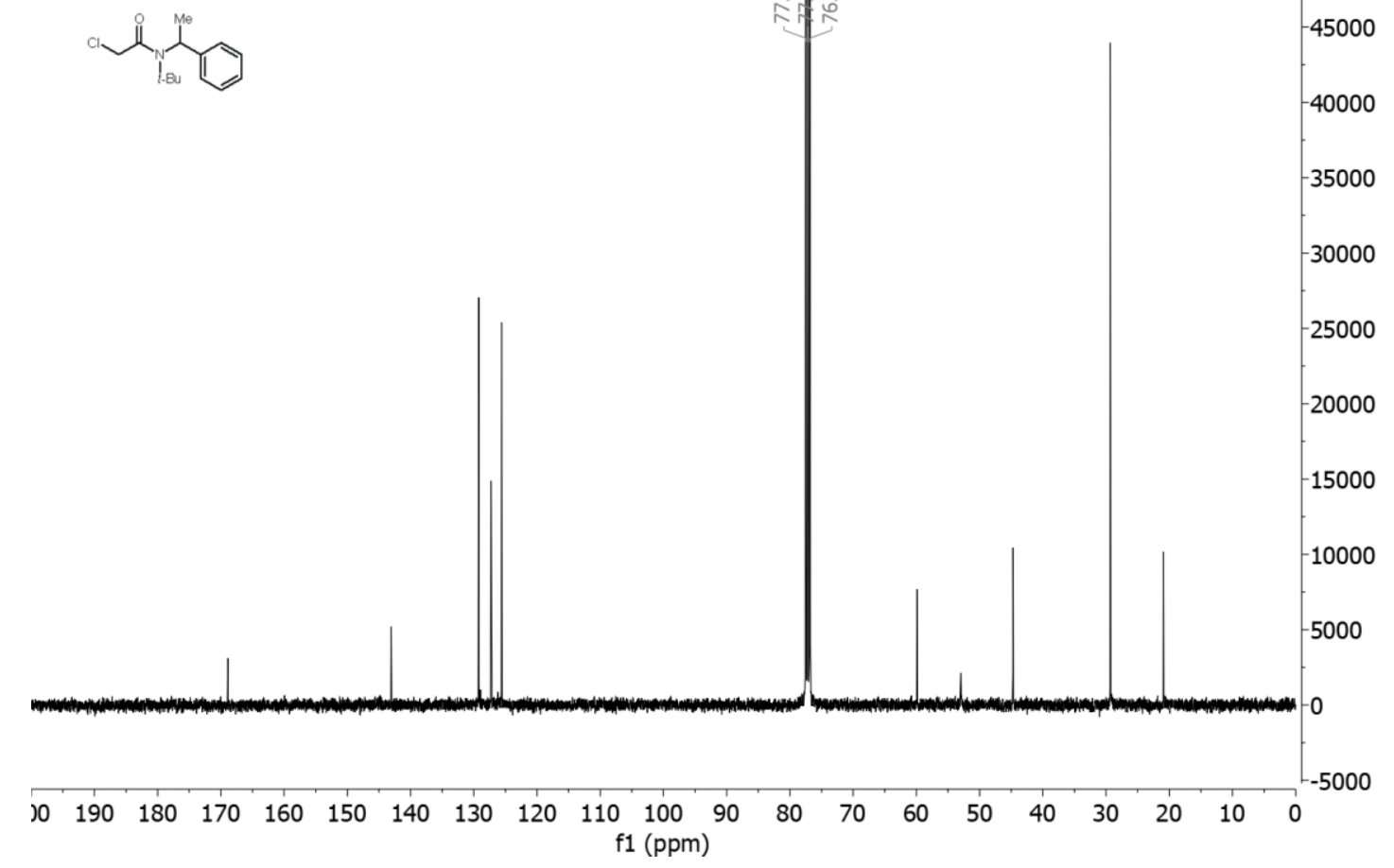

f1 (ppm) 
${ }^{1} \mathrm{H}$ NMR of $\mathbf{S 2 7}$

$600.18 \mathrm{MHz}$
$\mathrm{CDCl} 3 \mathrm{H} 1$

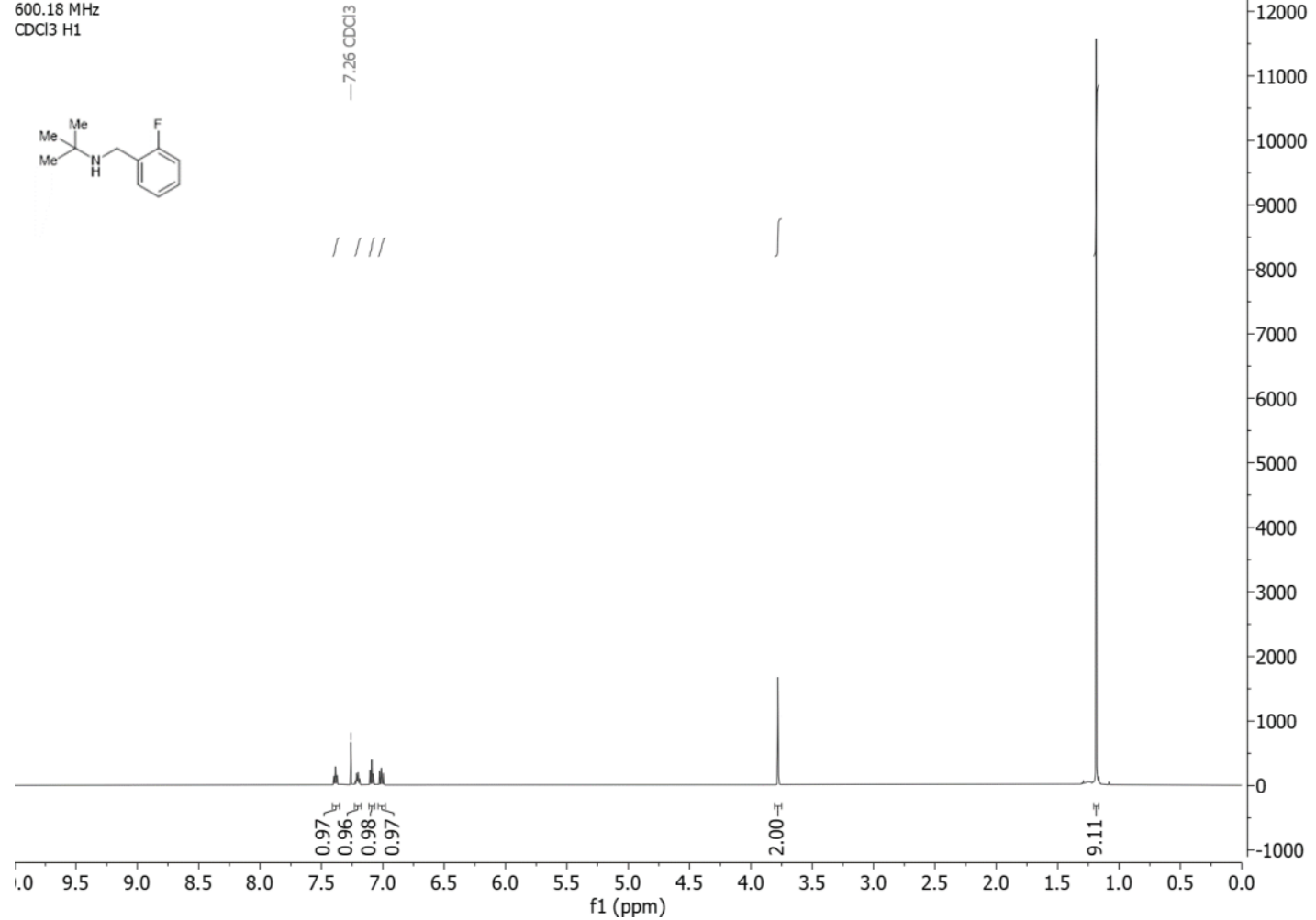

${ }^{13} \mathrm{C}$ NMR of $\mathbf{S 2 7}$

$150.93 \mathrm{MHz}$

CDCl3 $\mathrm{C} 13$

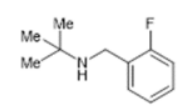

- IYOU0

$-18000$

$-17000$

$-16000$

$-15000$

$-14000$

$-13000$

$-12000$

$-11000$

$-10000$

$-9000$

$-8000$

$-7000$

$-6000$

$-5000$

$-4000$

$-3000$

$-2000$

$-1000$

$-1000$

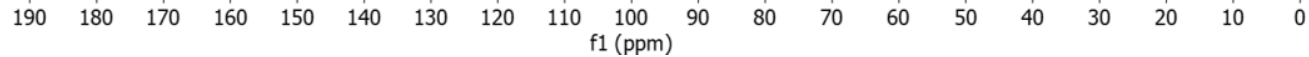




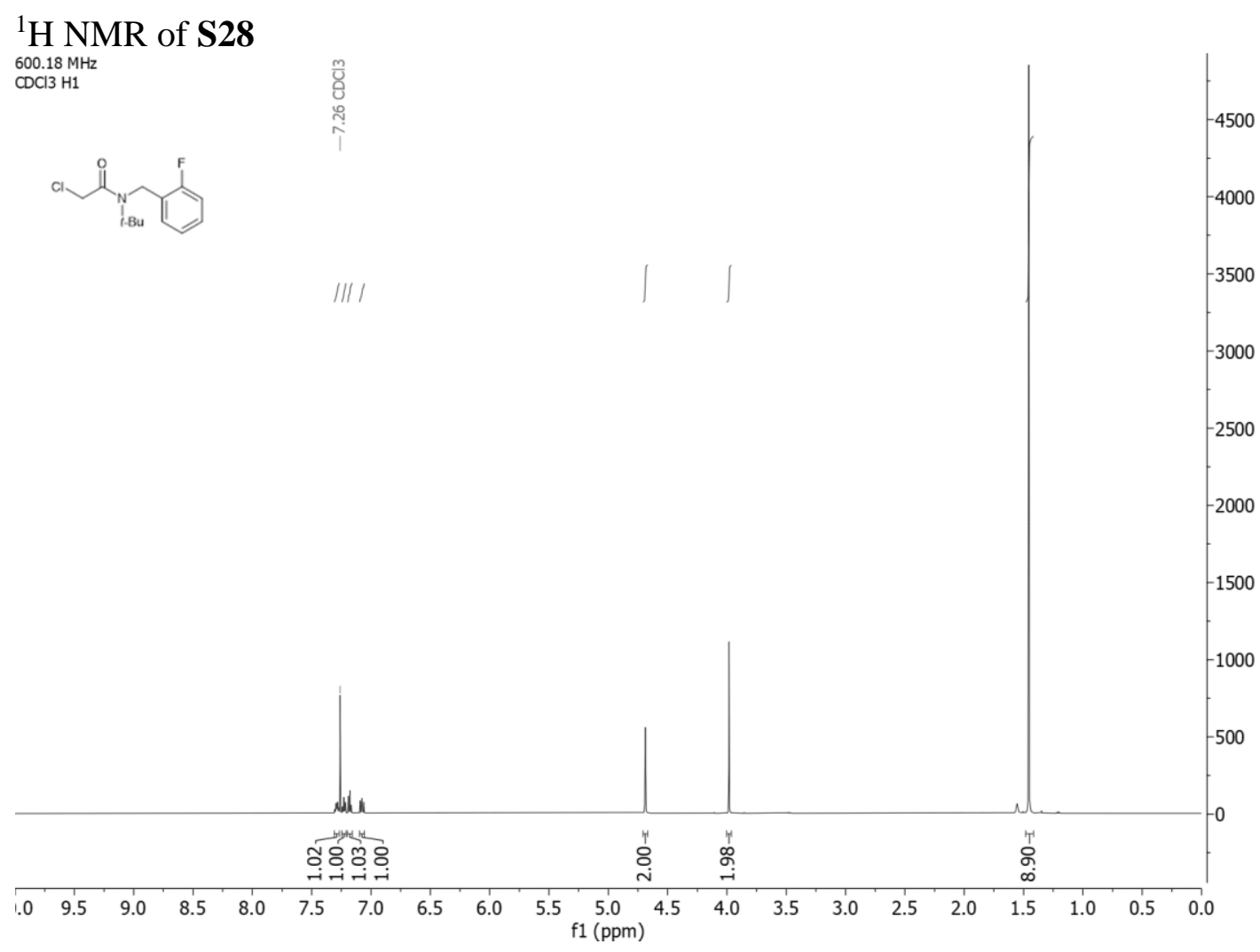

\section{${ }^{13} \mathrm{C}$ NMR of $\mathbf{S 2 8}$}

$150.93 \mathrm{MHz}$

$\mathrm{CDCl} 3 \mathrm{C} 13$

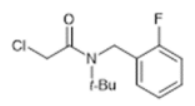

.



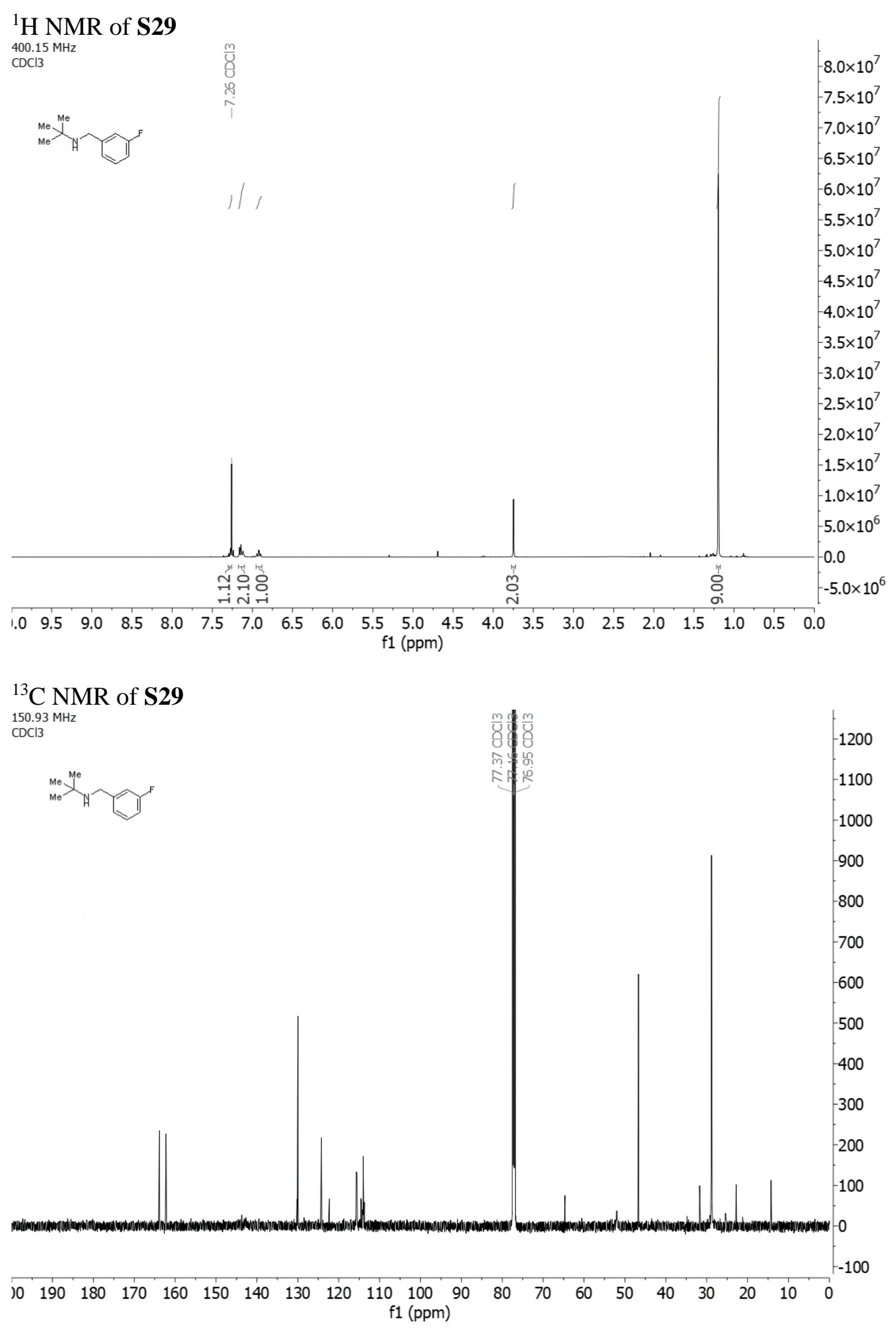
${ }^{1} \mathrm{H}$ NMR of $\mathbf{S 3 0}$

$399.75 \mathrm{MHz}$

cddl3<smiles>C[C@H](CCl)C(=O)Cc1cccc(I)c1</smiles>

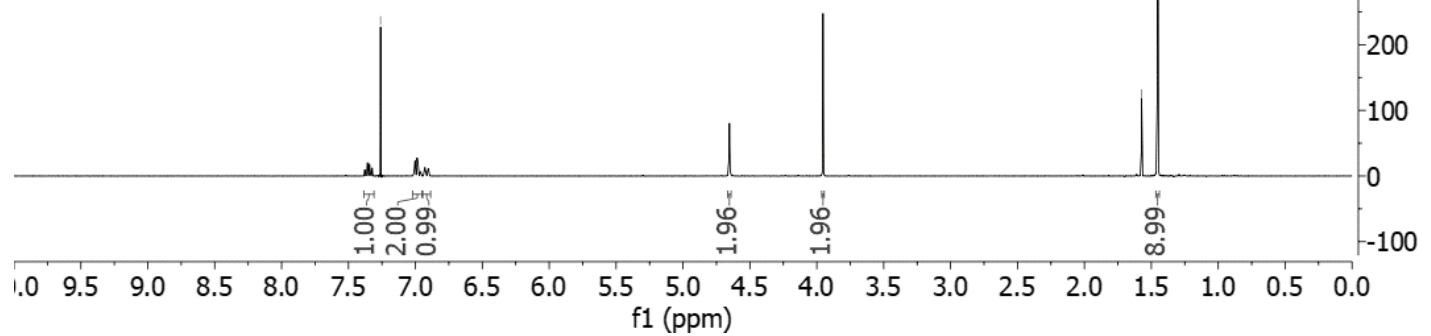

${ }^{13} \mathrm{C}$ NMR of $\mathbf{S 3 0}$ $150.93 \mathrm{MHz}$
$\mathrm{CDCl} 3$<smiles>Cc1cccc(CN(C)C(=O)CCl)c1</smiles>
f1 (ppm)

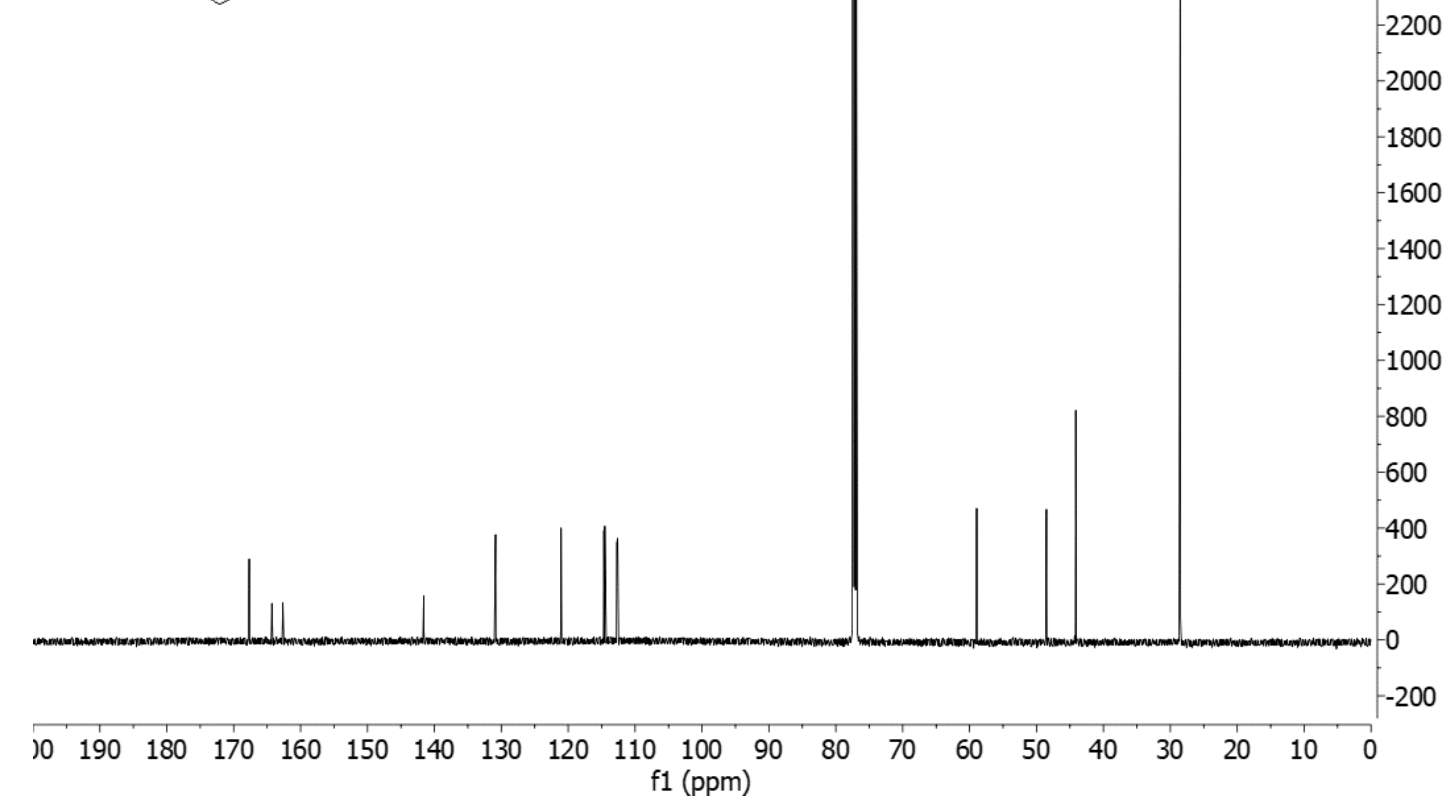


${ }^{1} \mathrm{H}$ NMR of $\mathbf{S 3 2}$

$600.18 \mathrm{MHz}$
$\mathrm{CDCl} 3 \mathrm{H} 1$

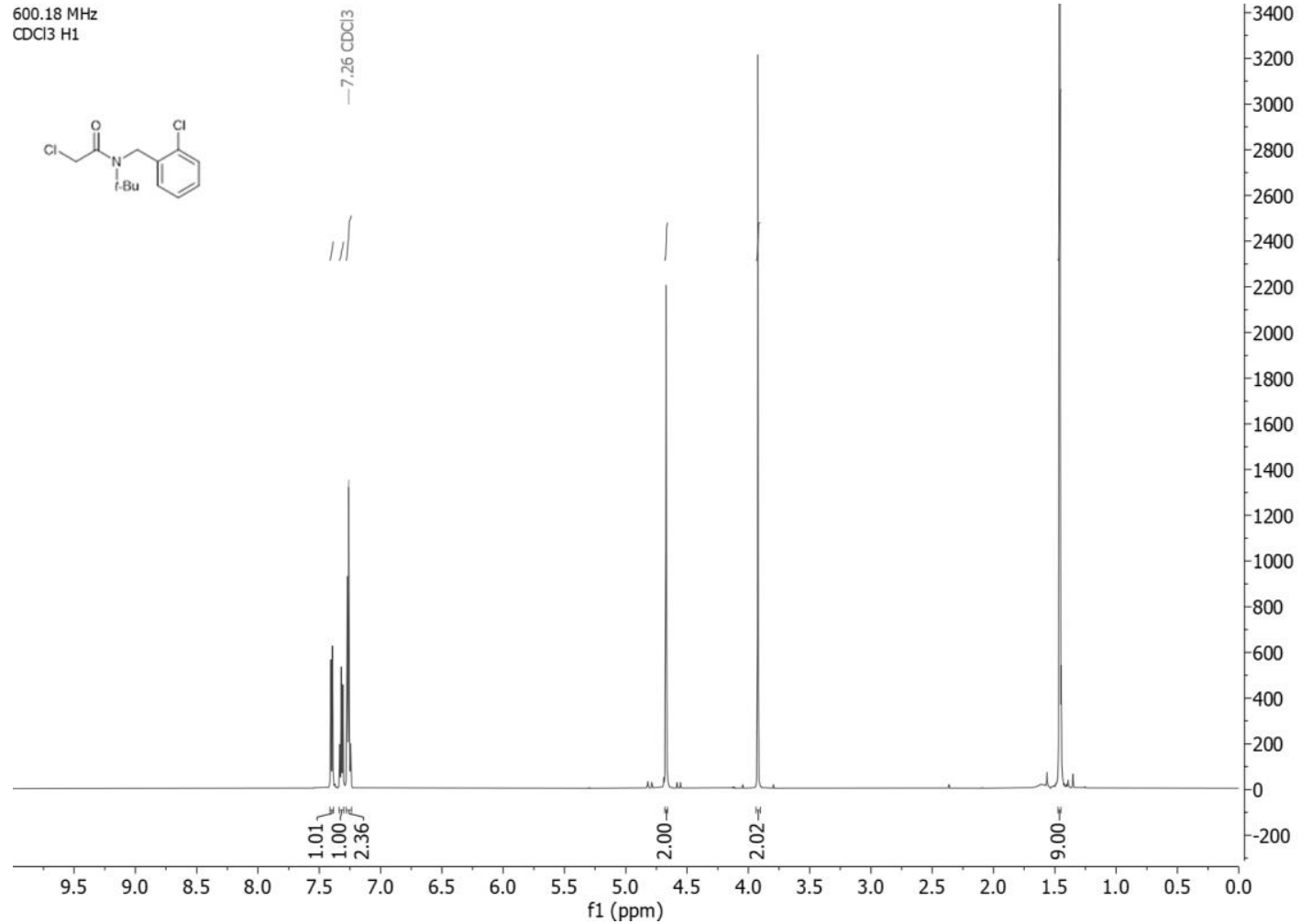

${ }^{13} \mathrm{C}$ NMR of $\mathbf{S 3 2}$

${ }_{150.93 \mathrm{MHz}}$ $\mathrm{CDCl3} \mathrm{C} 13$
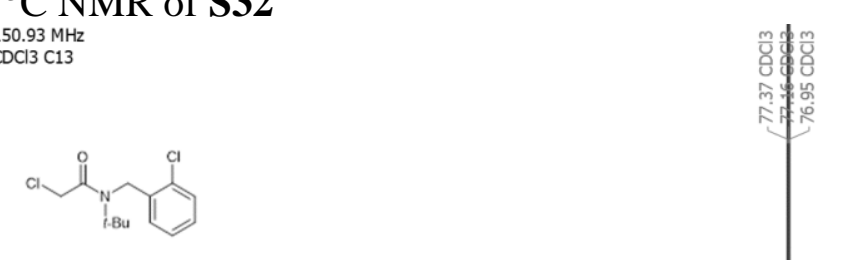
${ }^{1} \mathrm{H}$ NMR of $\mathbf{S 3 4}$

$400.15 \mathrm{MHz}$
$\mathrm{CDCl} 3$

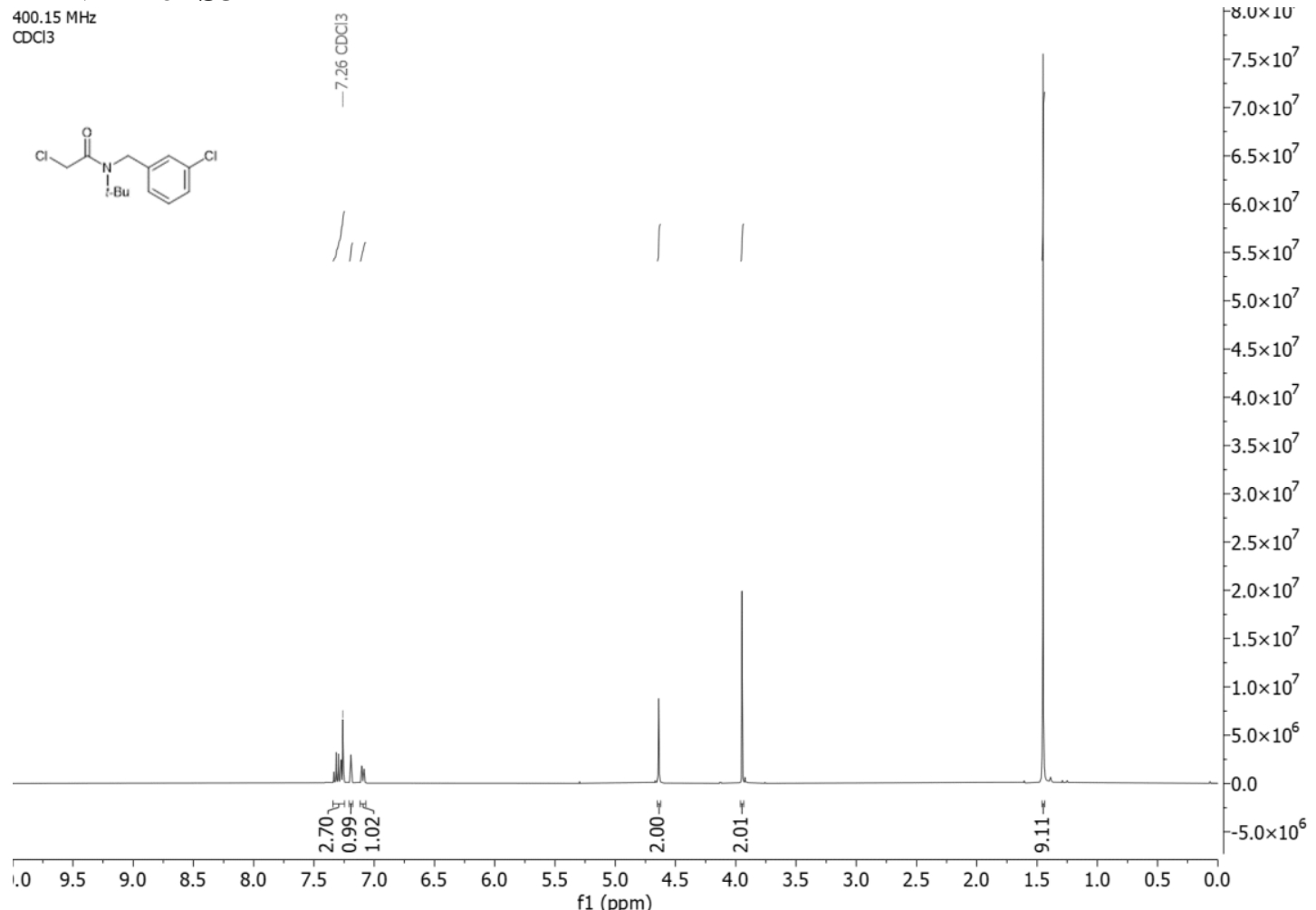

${ }^{13} \mathrm{C}$ NMR of $\mathbf{S 3 4}$

${ }_{100.63 \mathrm{MHz}}$

$\mathrm{CDCl} 3$

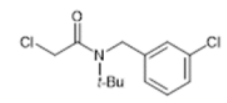

f1 (ppm)

2000000

$-1900000$

$-1800000$

$-1700000$

$-1600000$

$-1500000$

$-1400000$

$-1300000$

$-1200000$

$-1100000$

$-1000000$

$-900000$

$-800000$

$-700000$

$-600000$ 

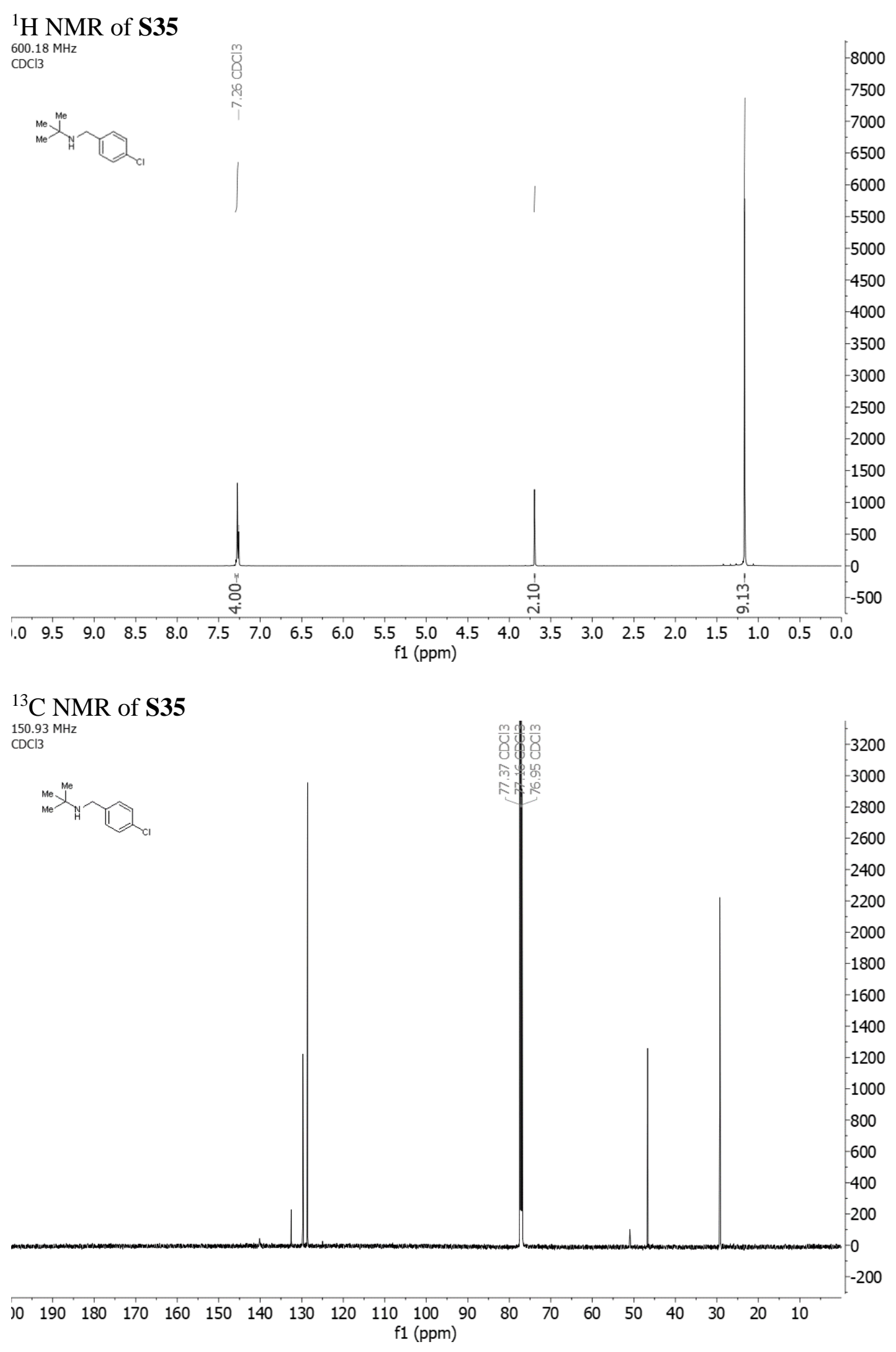
${ }^{1} \mathrm{H}$ NMR of $\mathbf{S 3 7}$

${ }_{00013}^{400.15} \mathrm{MHz}$

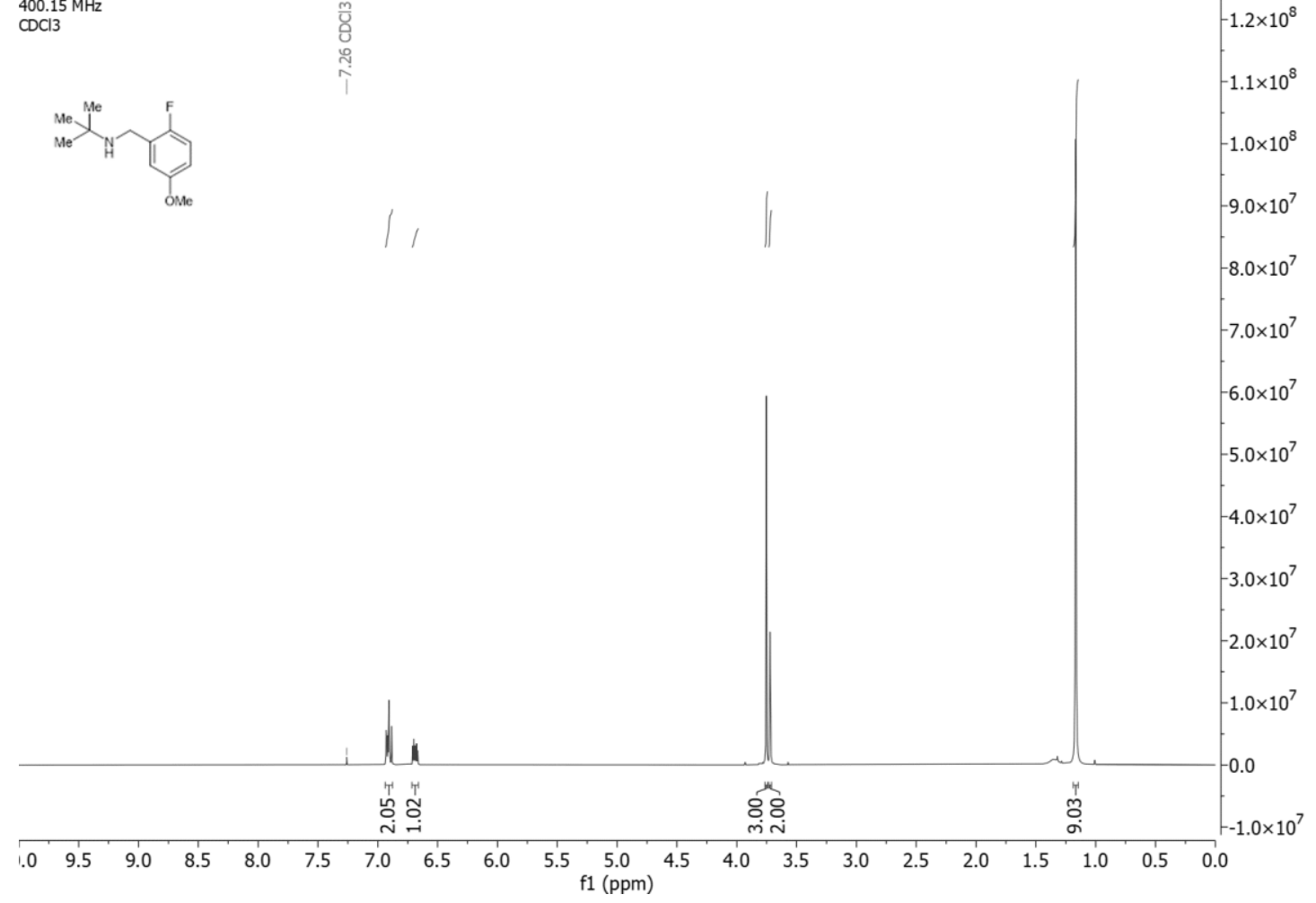

${ }^{13} \mathrm{C}$ NMR of $\mathbf{S 3 7}$

${ }^{100.63 \mathrm{MHz}}$

$\mathrm{CDCl} 3$
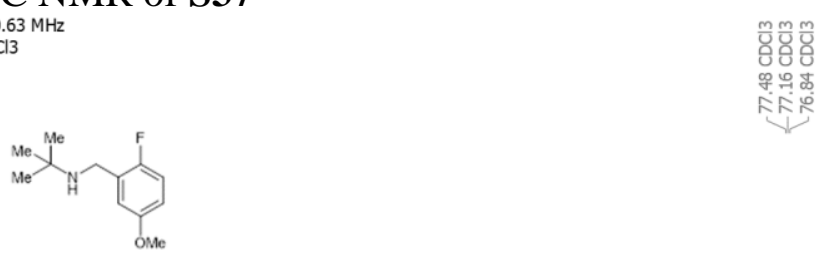

$-20000000$

$-19000000$

$-18000000$

$-17000000$

$-16000000$

$-15000000$

$-14000000$

$-13000000$

$-12000000$

$-11000000$

$-10000000$

$-9000000$

$-8000000$

$-7000000$

$-6000000$

$-5000000$

$-4000000$

$-3000000$

$-2000000$

$-1000000$

$-1000000$

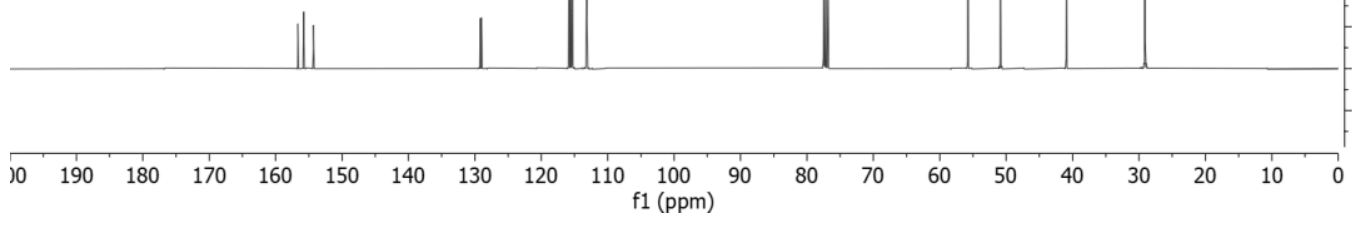


${ }^{1} \mathrm{H}$ NMR of $\mathbf{S 3 8}$

$400.15 \mathrm{MHz}$

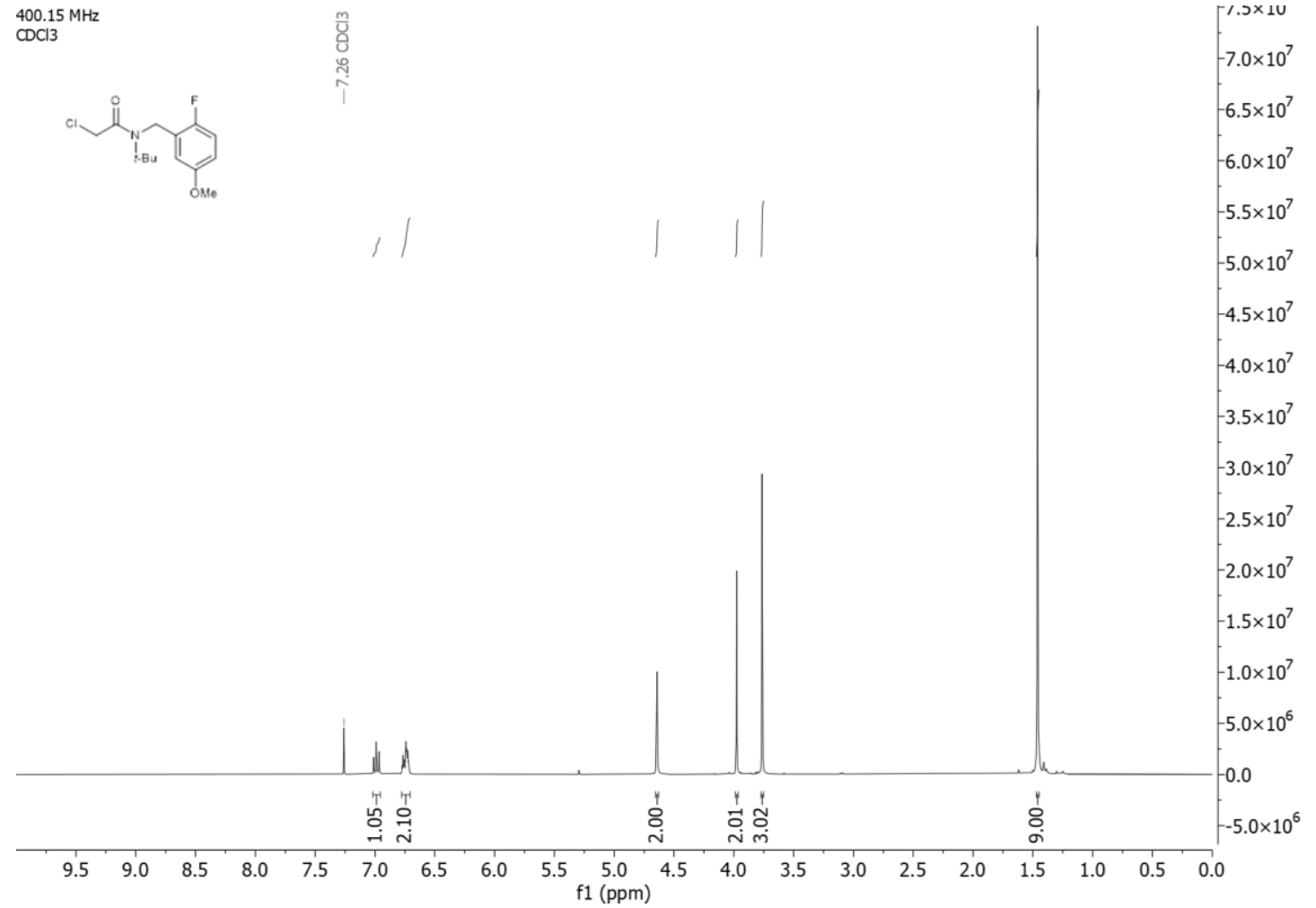

${ }^{13} \mathrm{C}$ NMR of $\mathbf{S 3 8}$

$100.63 \mathrm{MHz}$

CDCl3 F19

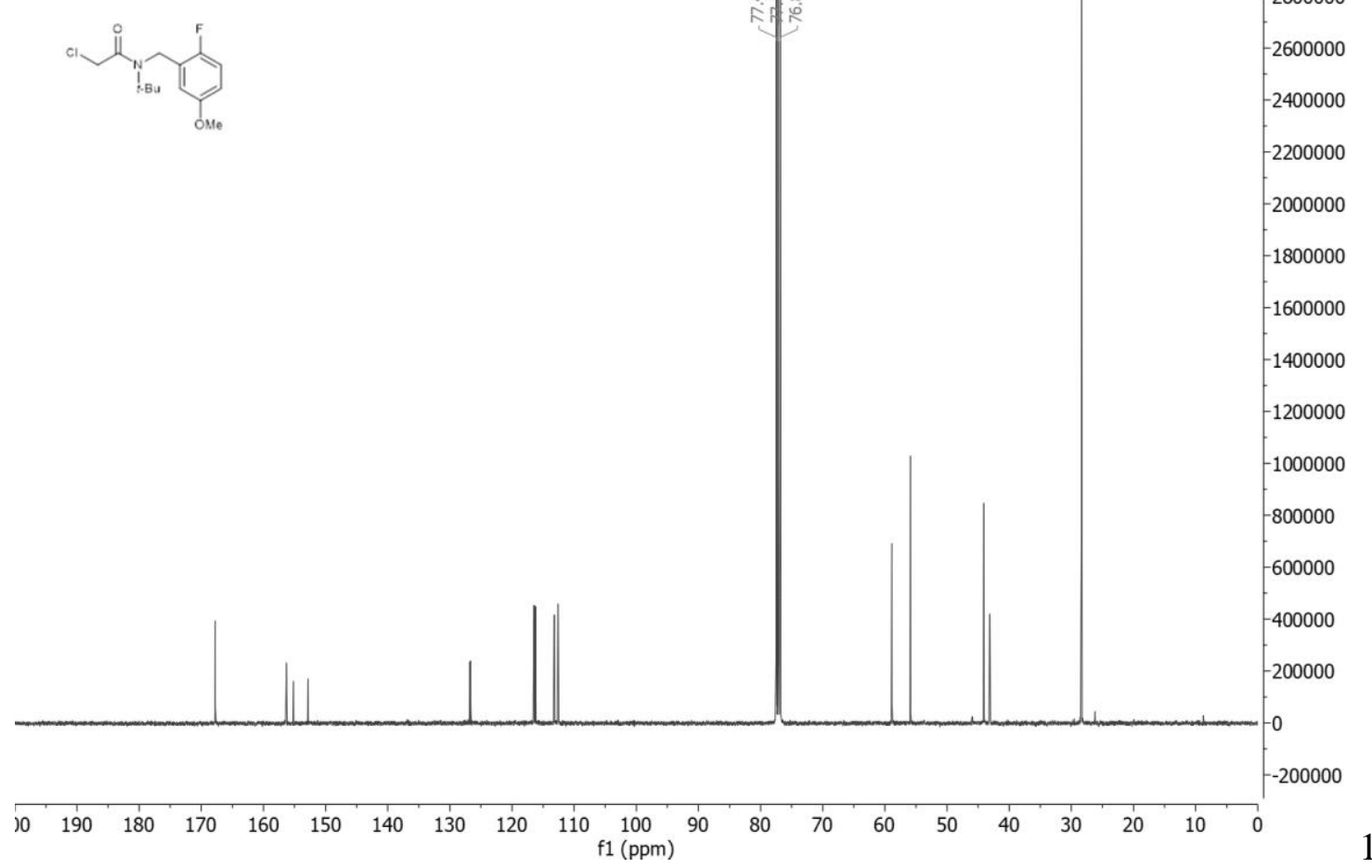


${ }^{1} \mathrm{H}$ NMR of $\mathbf{S 4 1}$

${ }_{000 \mathrm{CCl}}^{60.18 \mathrm{MHz}}$

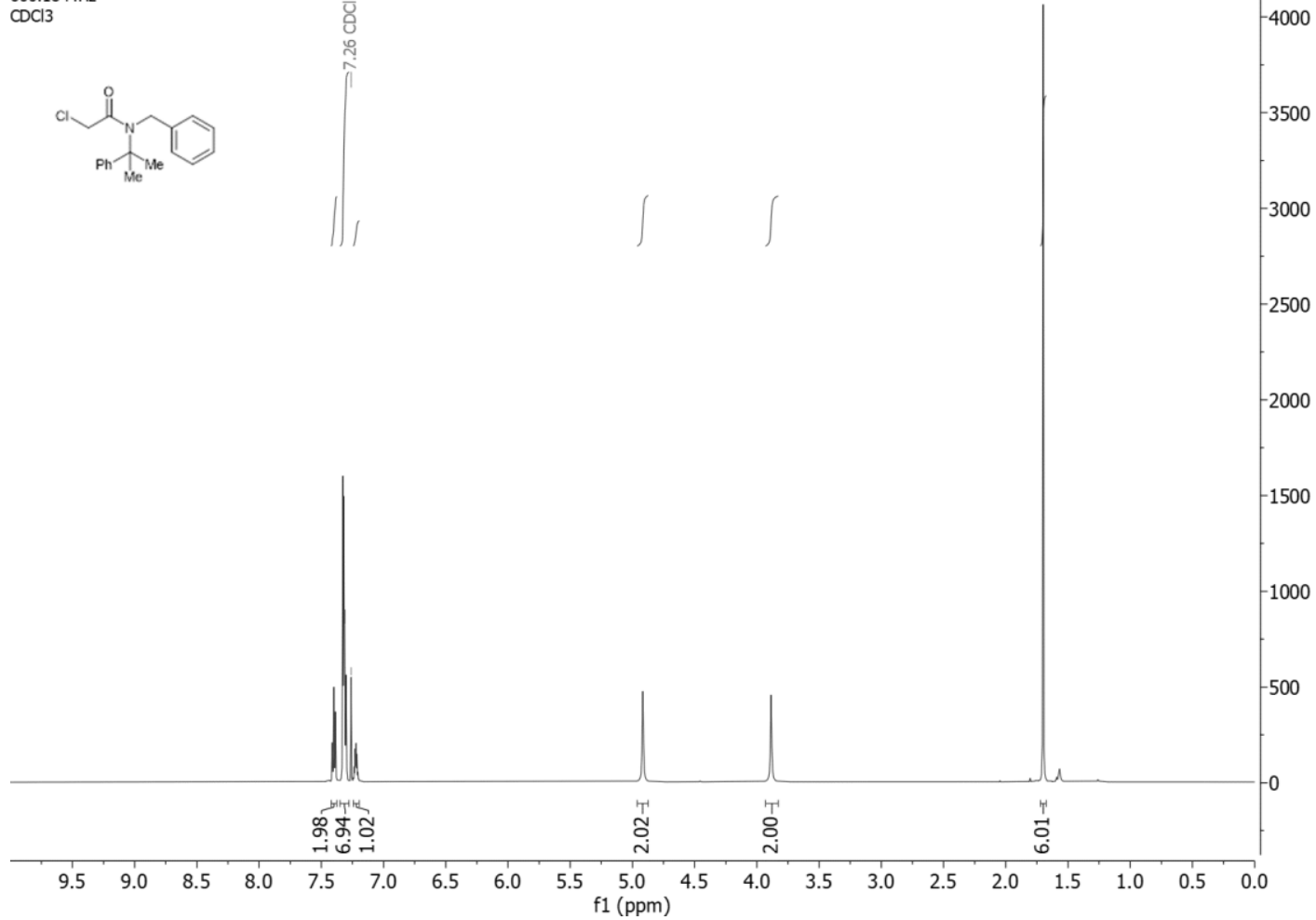

${ }^{13} \mathrm{C}$ NMR of $\mathbf{S 4 1}$

$150.93 \mathrm{MHz}$

$\mathrm{CDCl} 3$

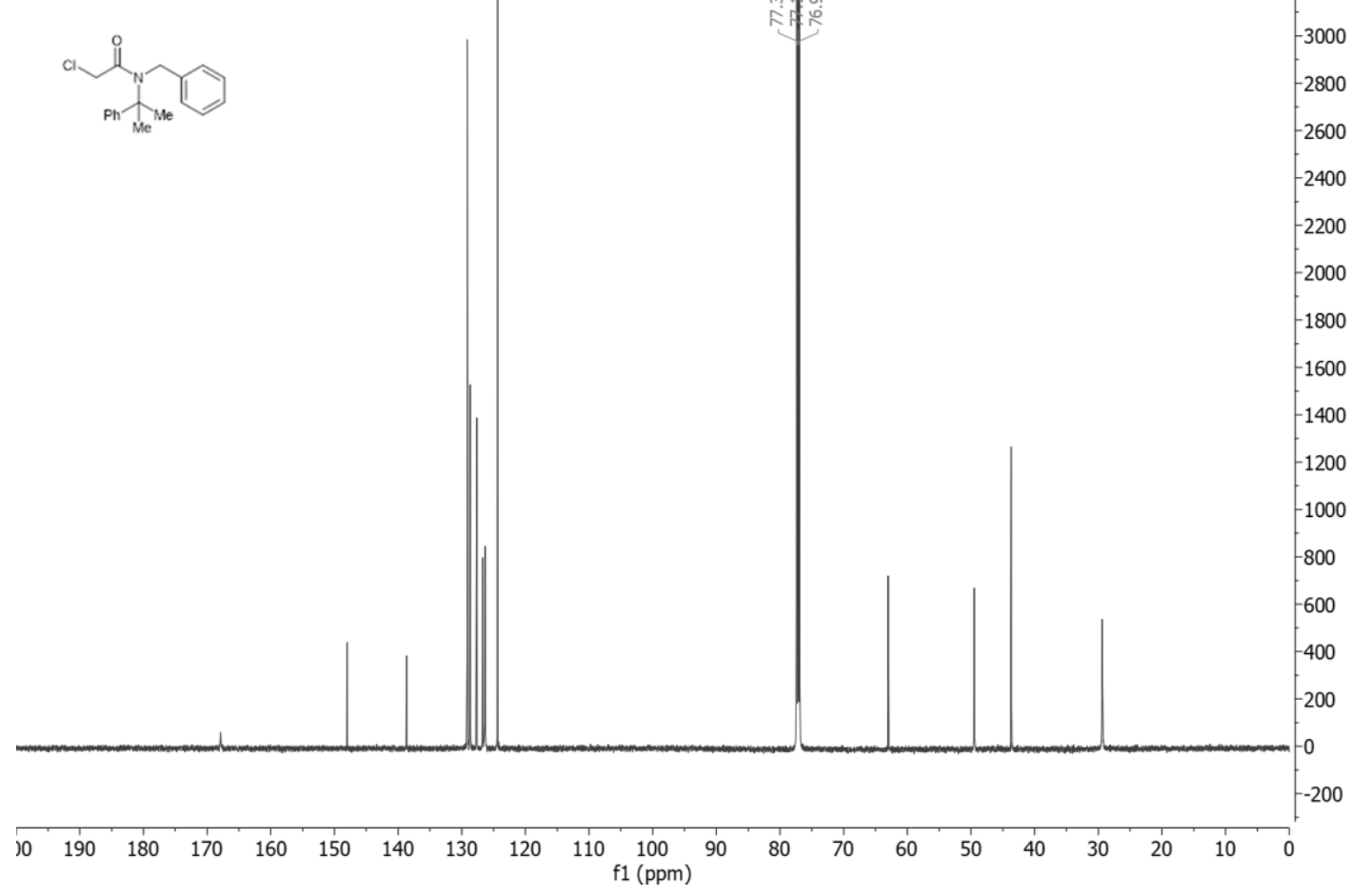

S-85 

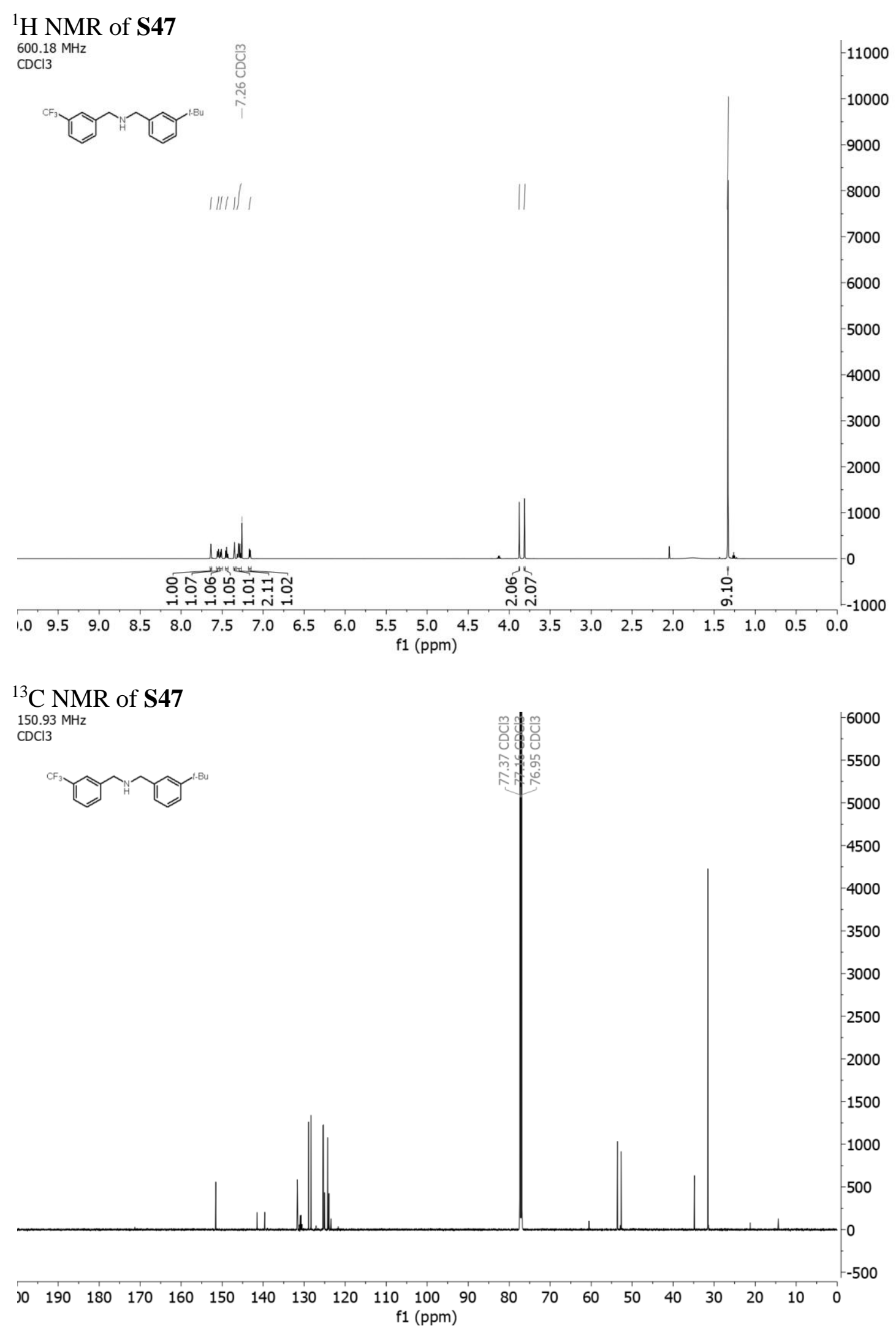


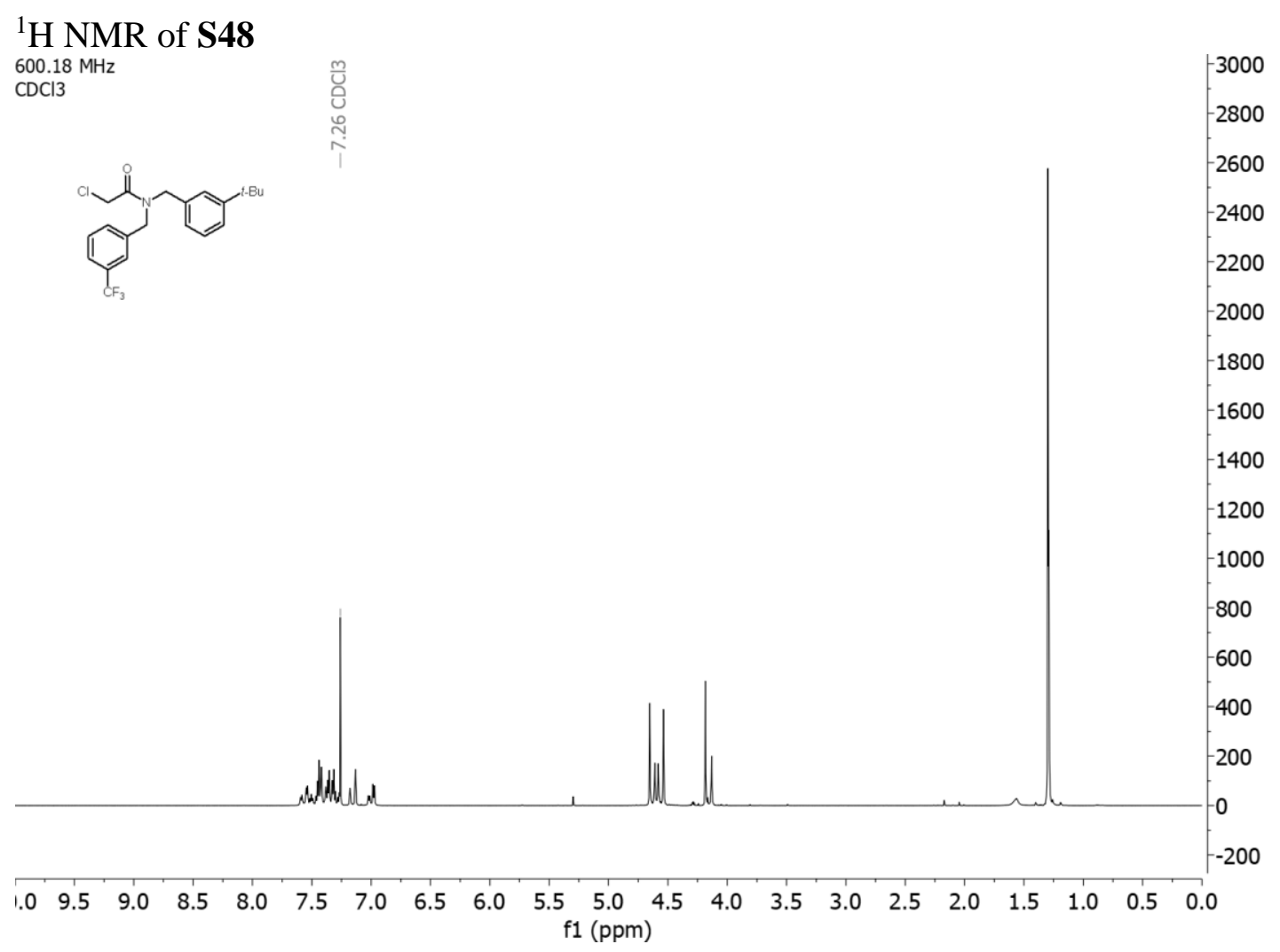

${ }^{13} \mathrm{C}$ NMR of $\mathbf{S 4 8}$ $150.93 \mathrm{MHz}$ CDCl3

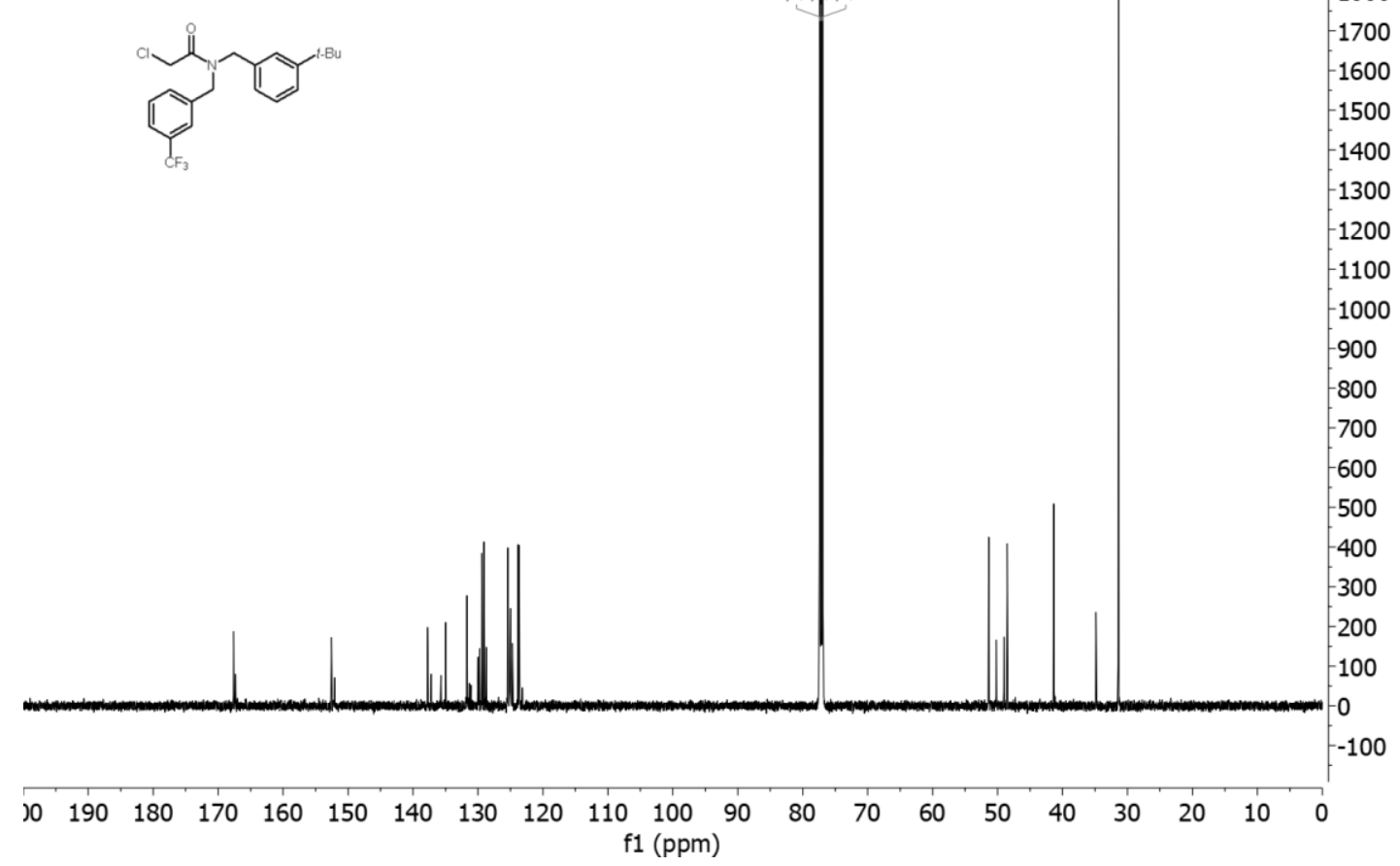


${ }^{1} \mathrm{H}$ NMR of $\mathbf{S 4 9}$

$400.15 \mathrm{MHz}$
$\mathrm{CDCl} 3$
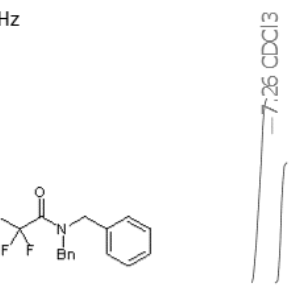

16000000

$-15000000$

$-14000000$

$-13000000$

$-12000000$

$-11000000$

$-10000000$

$-9000000$

$-8000000$

$-7000000$

$-6000000$

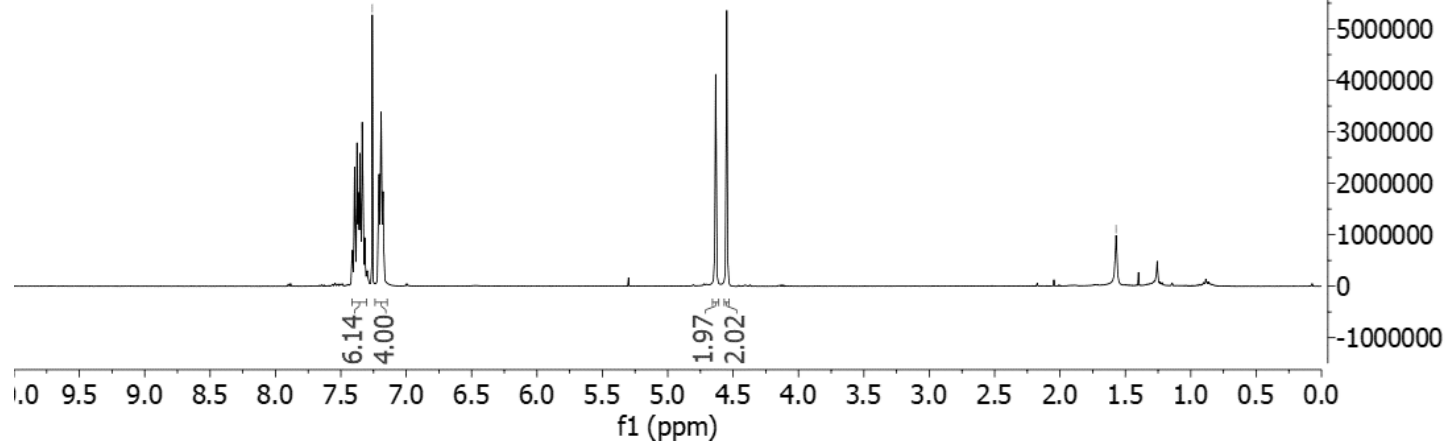

${ }^{13} \mathrm{C}$ NMR of $\mathbf{S 4 9}$ $150.93 \mathrm{MHz}$
$\mathrm{CDCl} 3$

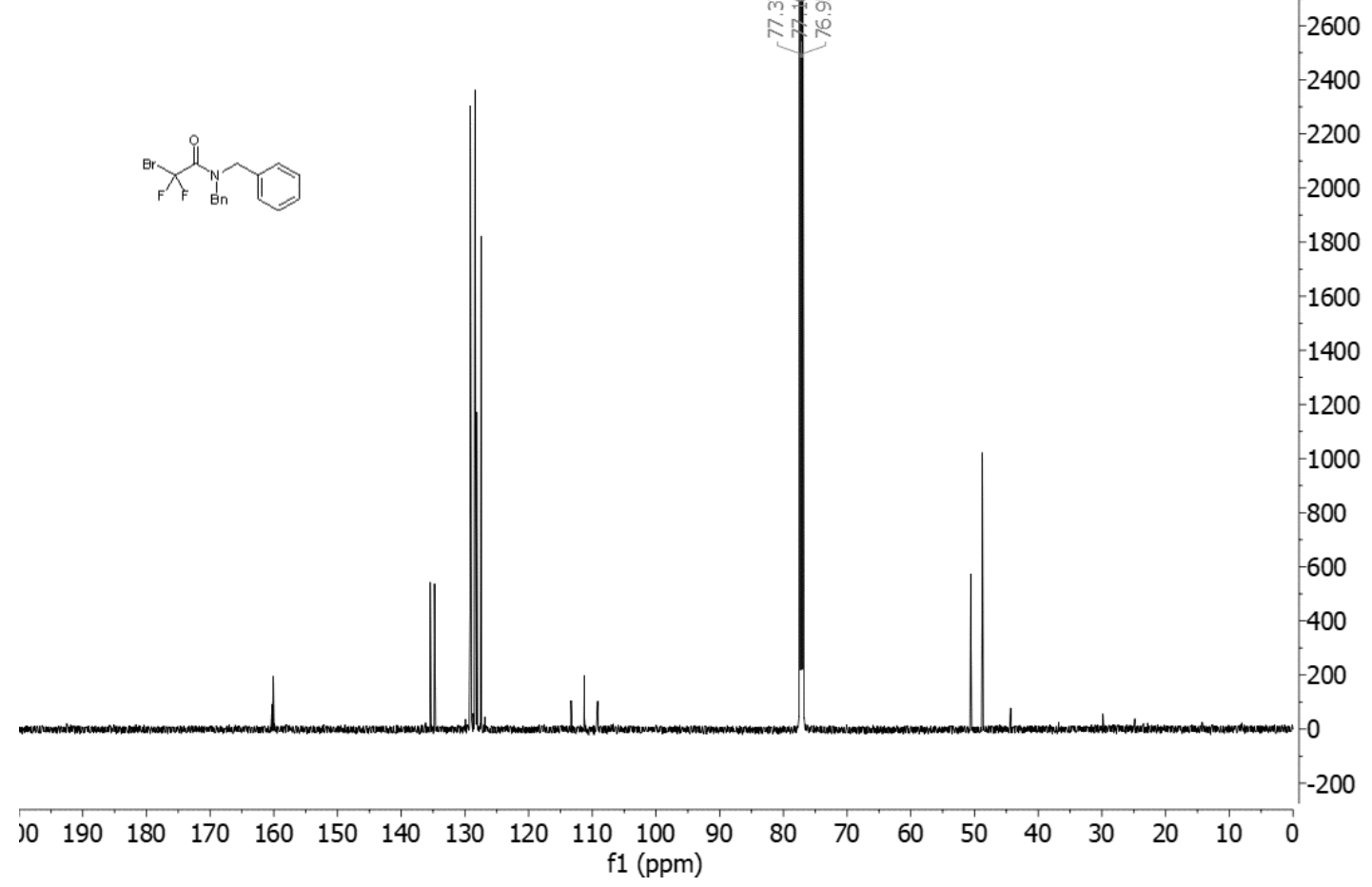


${ }^{1} \mathrm{H}$ NMR of 1

$400.15 \mathrm{MHz}$

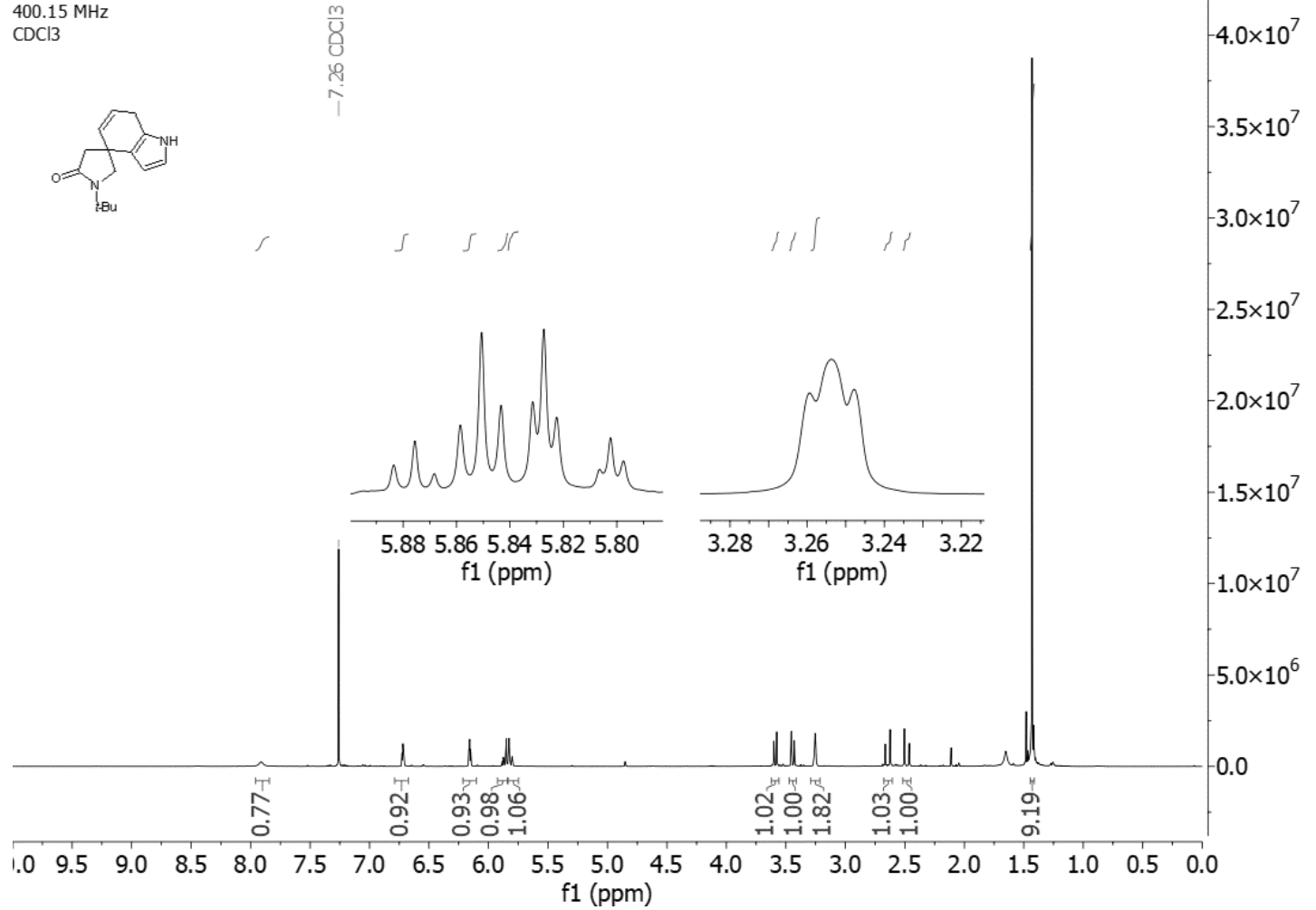

${ }^{13} \mathrm{C}$ NMR of 1

$150.93 \mathrm{MHz}$

$\mathrm{CDCl} 3$
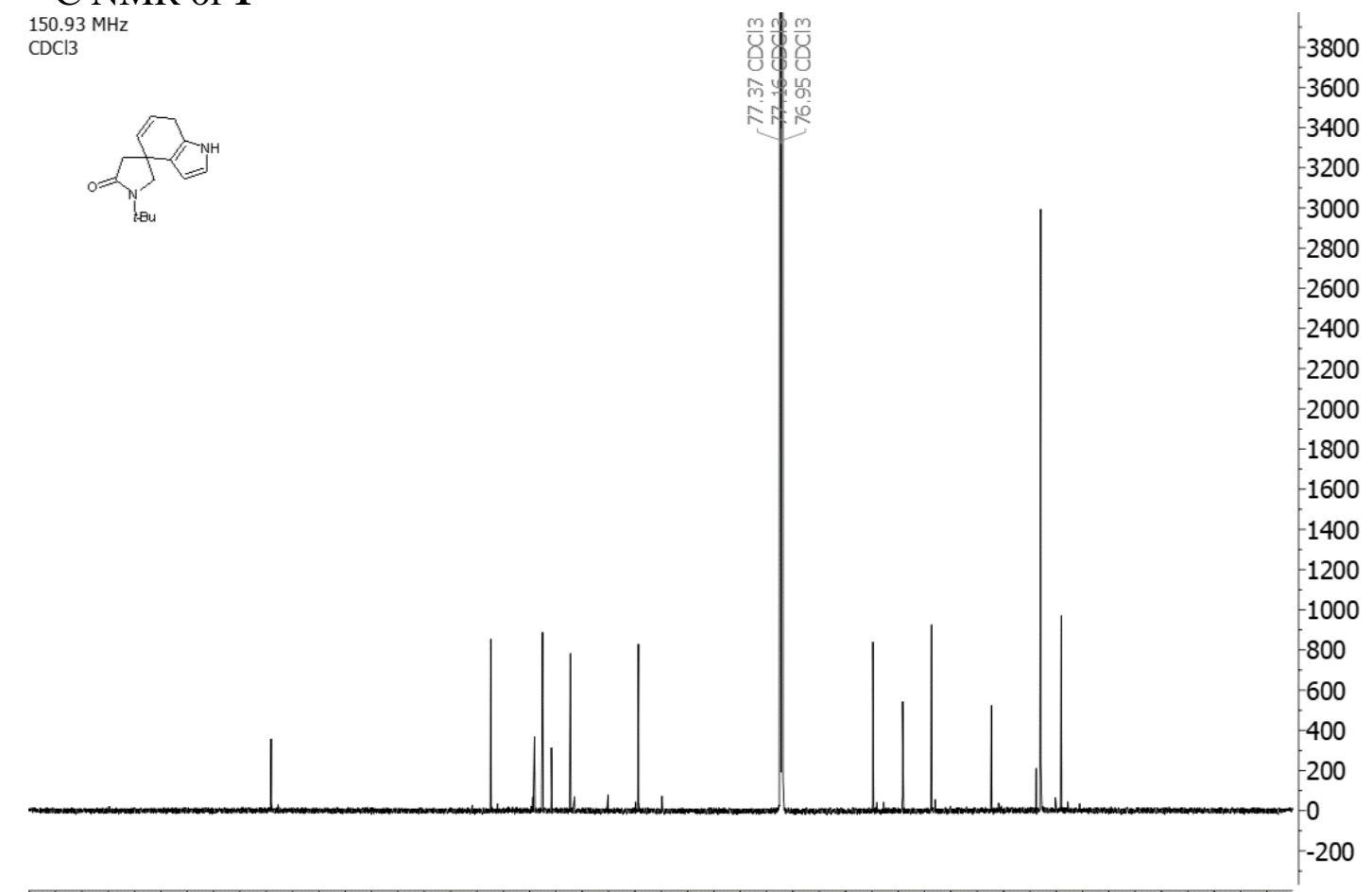

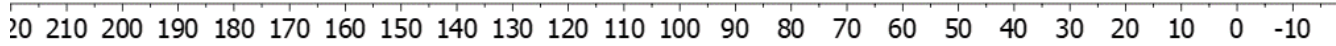
f1 (ppm) 
${ }^{1} \mathrm{H}$ NMR of 2

$600.18 \mathrm{MHz}$

$\mathrm{CDCl} 3$
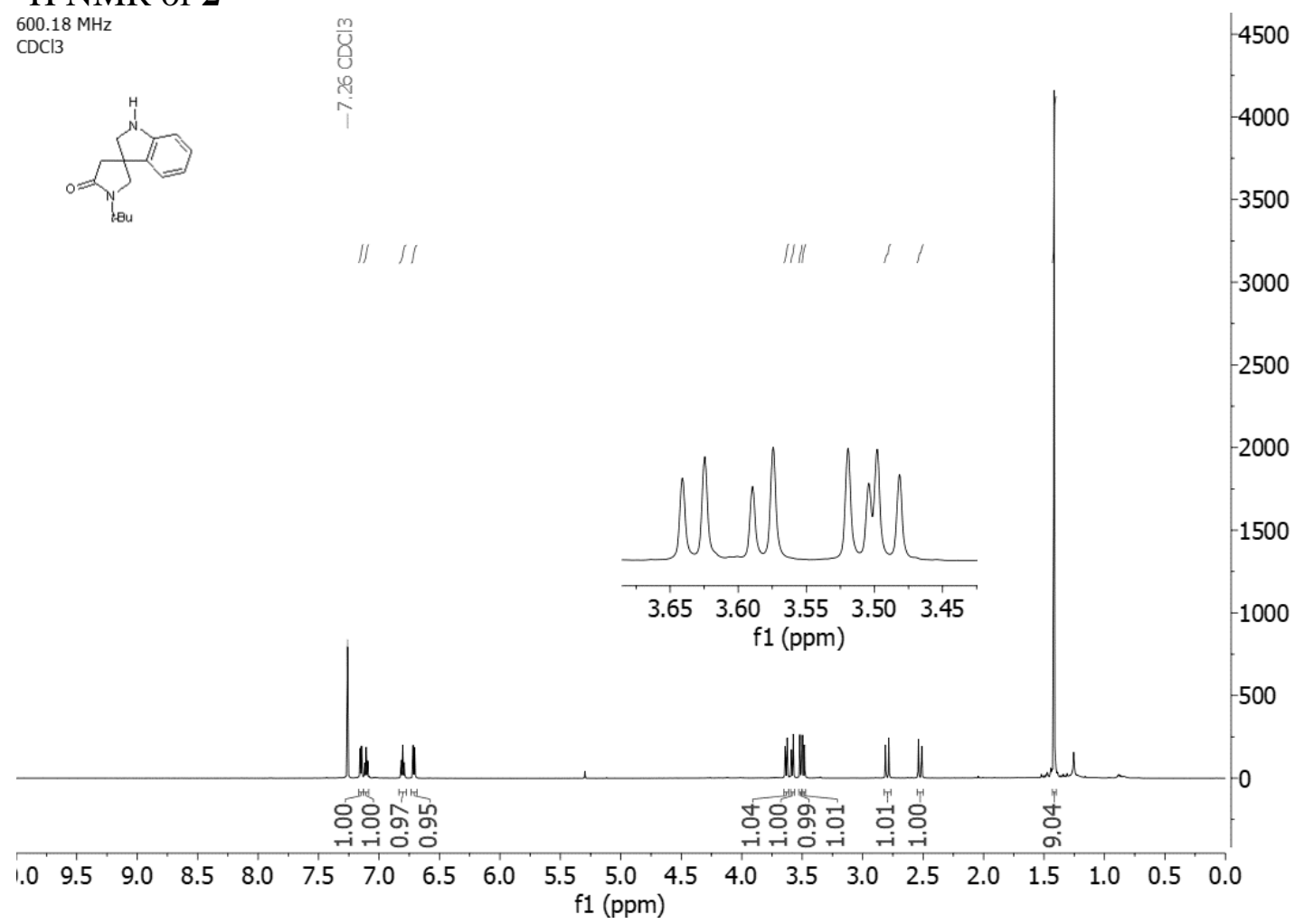

\section{${ }^{13} \mathrm{C}$ NMR of 2} $150.93 \mathrm{MHz}$ $\mathrm{CDCl} 3$

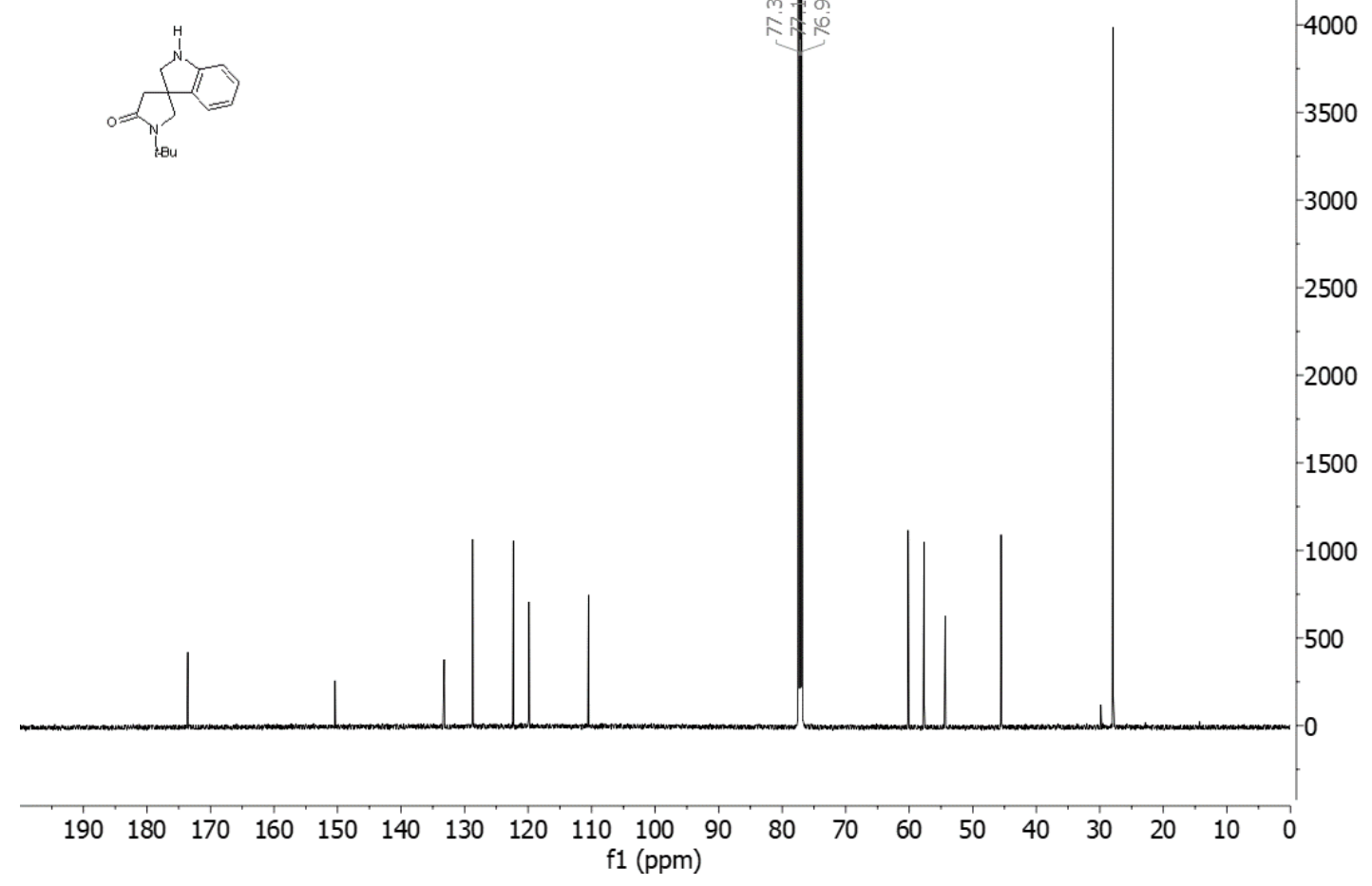


${ }^{1} \mathrm{H}$ NMR of 3

$500.29 \mathrm{MHz}$

cdcl3

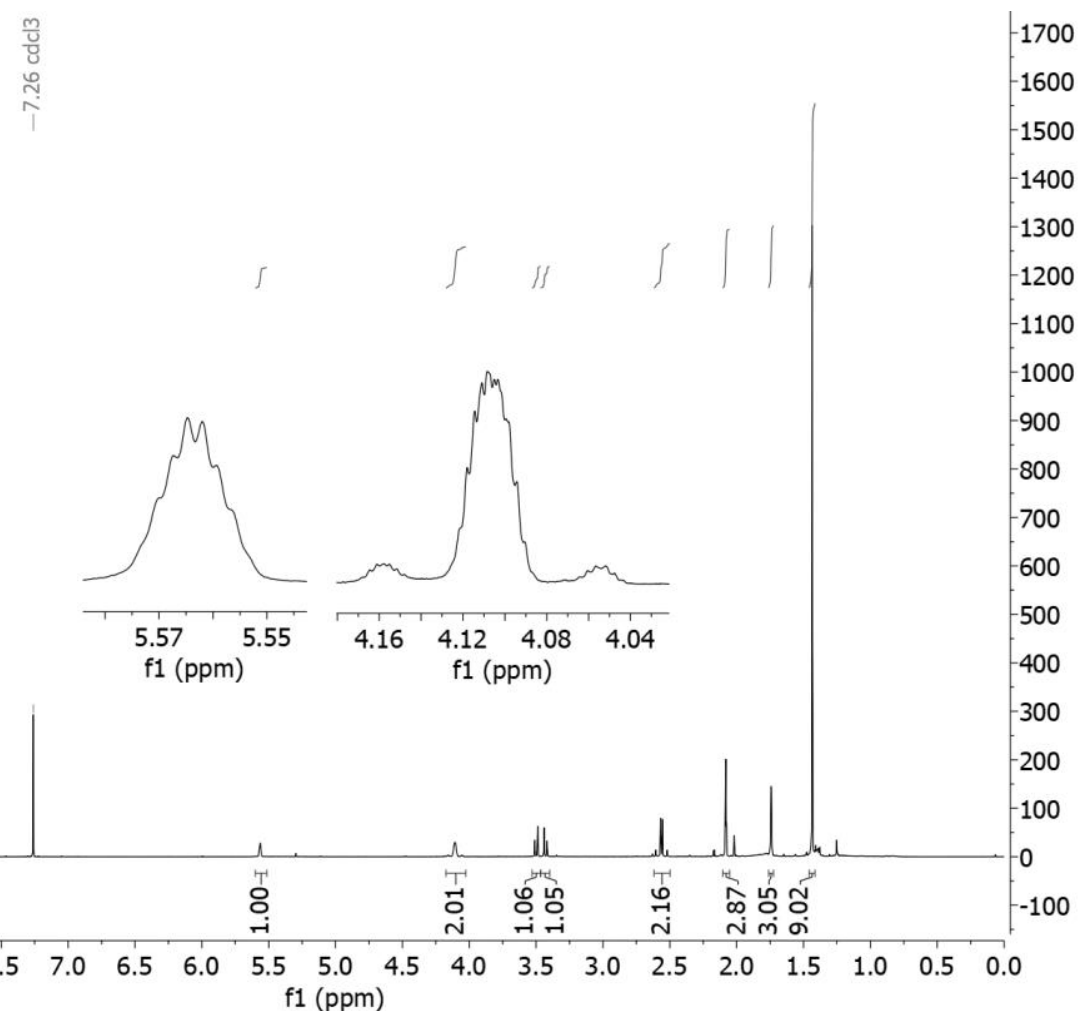

${ }^{13} \mathrm{C}$ NMR of 3

$100.54 \mathrm{MHz}$

$\mathrm{CDCl} 3$

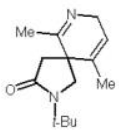

f1 (ppm)

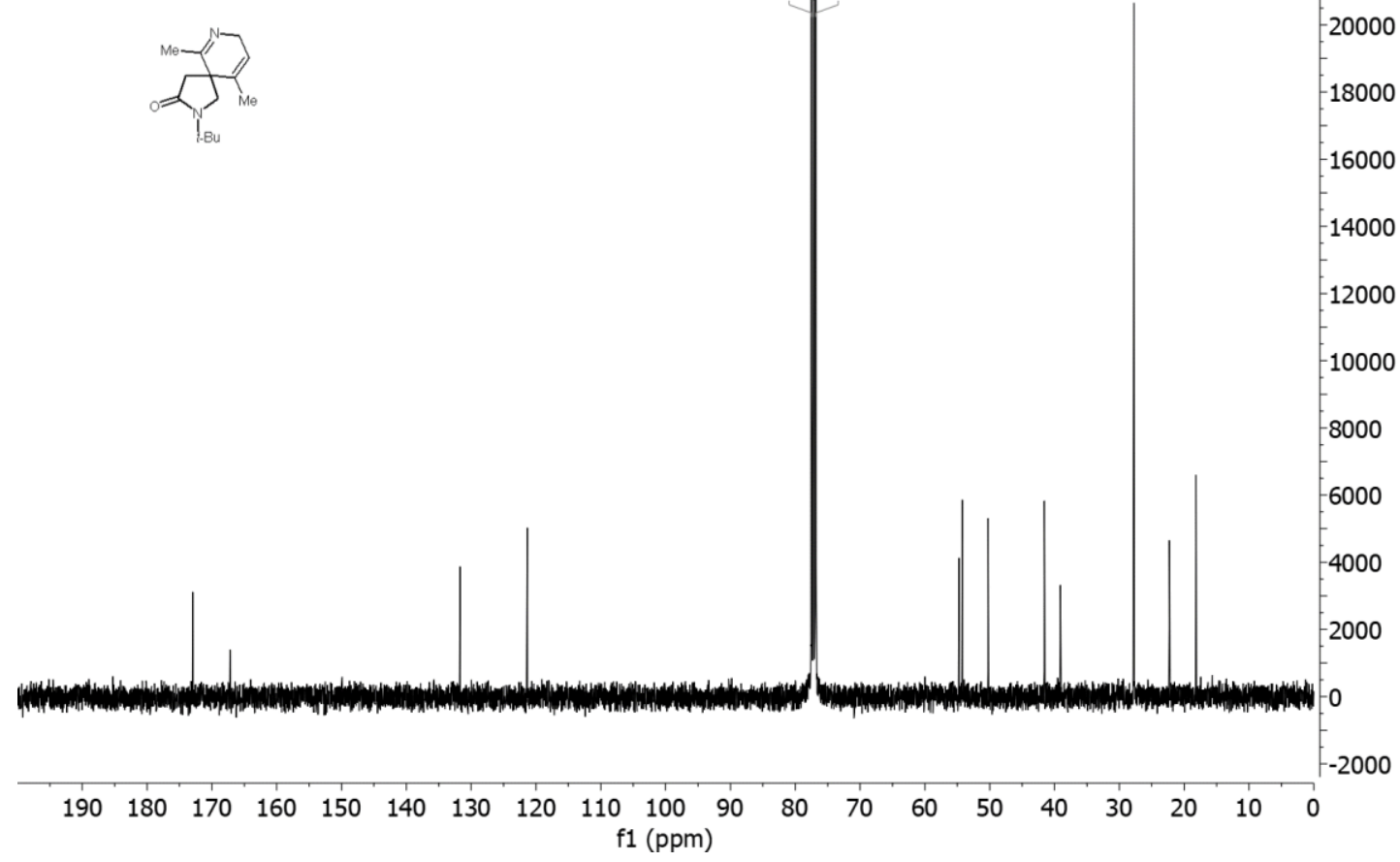




\section{${ }^{1} \mathrm{H}$ NMR of 4}

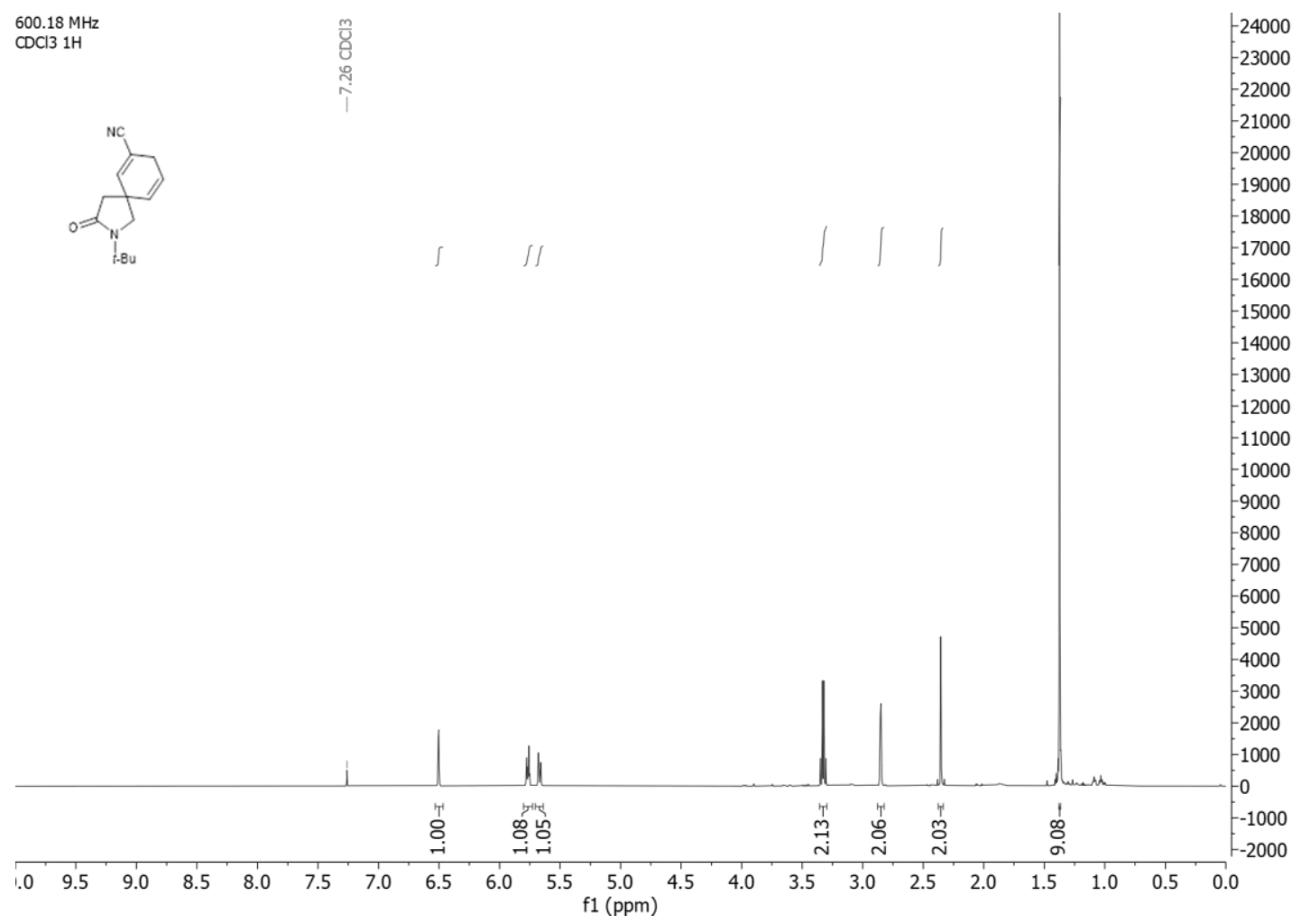

\section{${ }^{13} \mathrm{C}$ NMR of 4}

$150.93 \mathrm{MHz}$ $\mathrm{CDCl} 3$ 13C
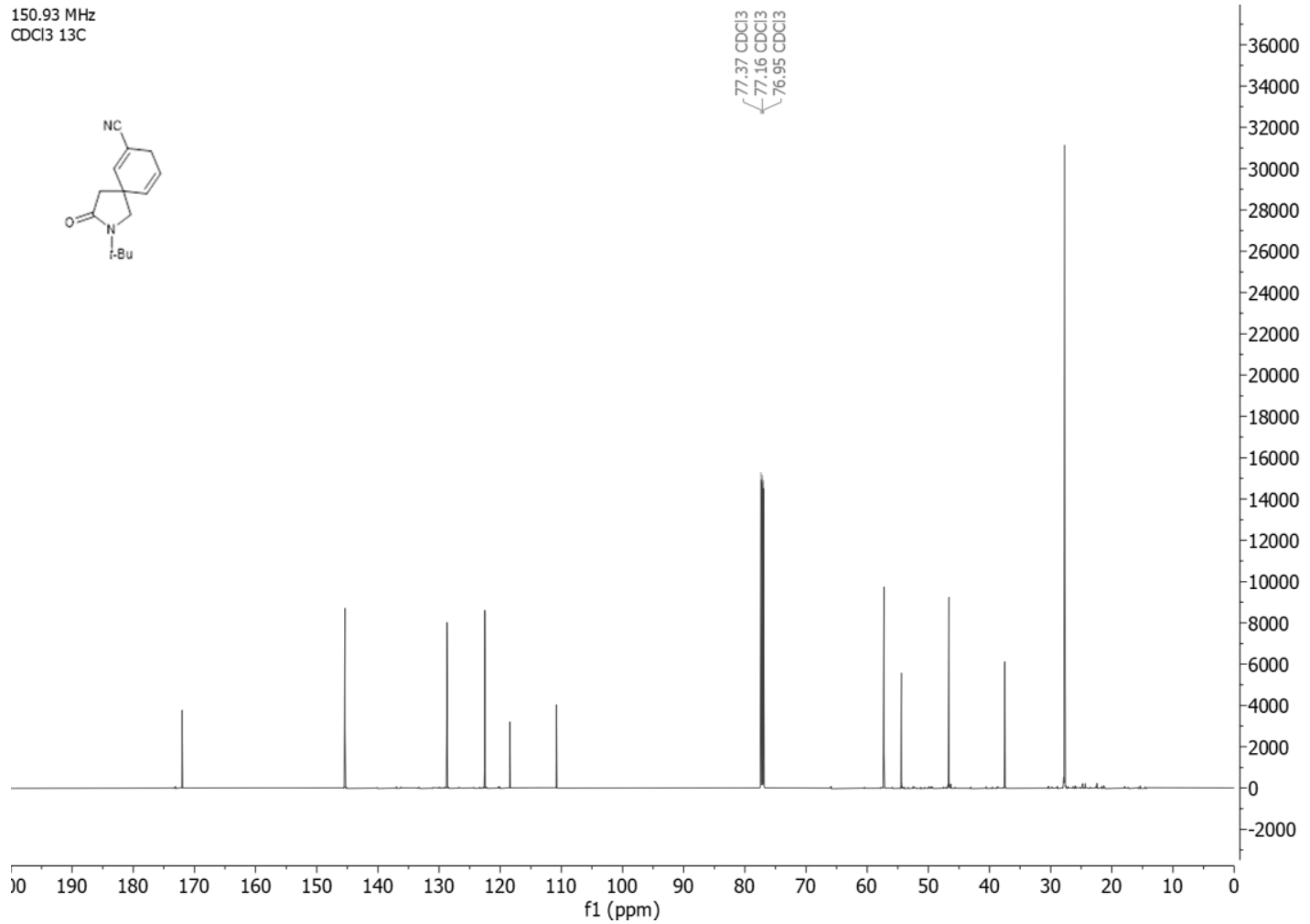
${ }^{1} \mathrm{H}$ NMR of 5

$400.15 \mathrm{MHz}$

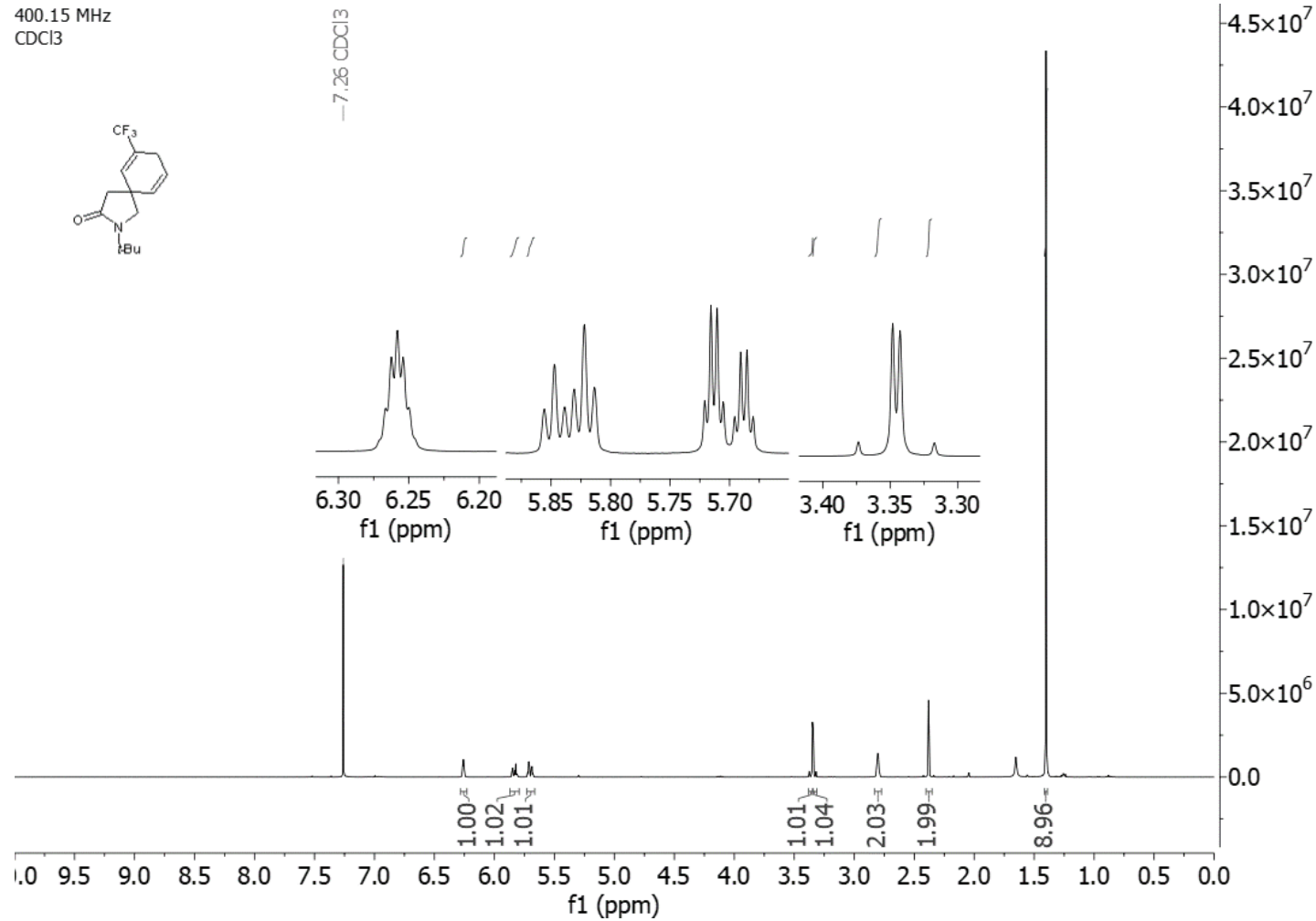

${ }^{13} \mathrm{C}$ NMR of 5

$150.93 \mathrm{MHz}$

$\mathrm{CDCl} 3$

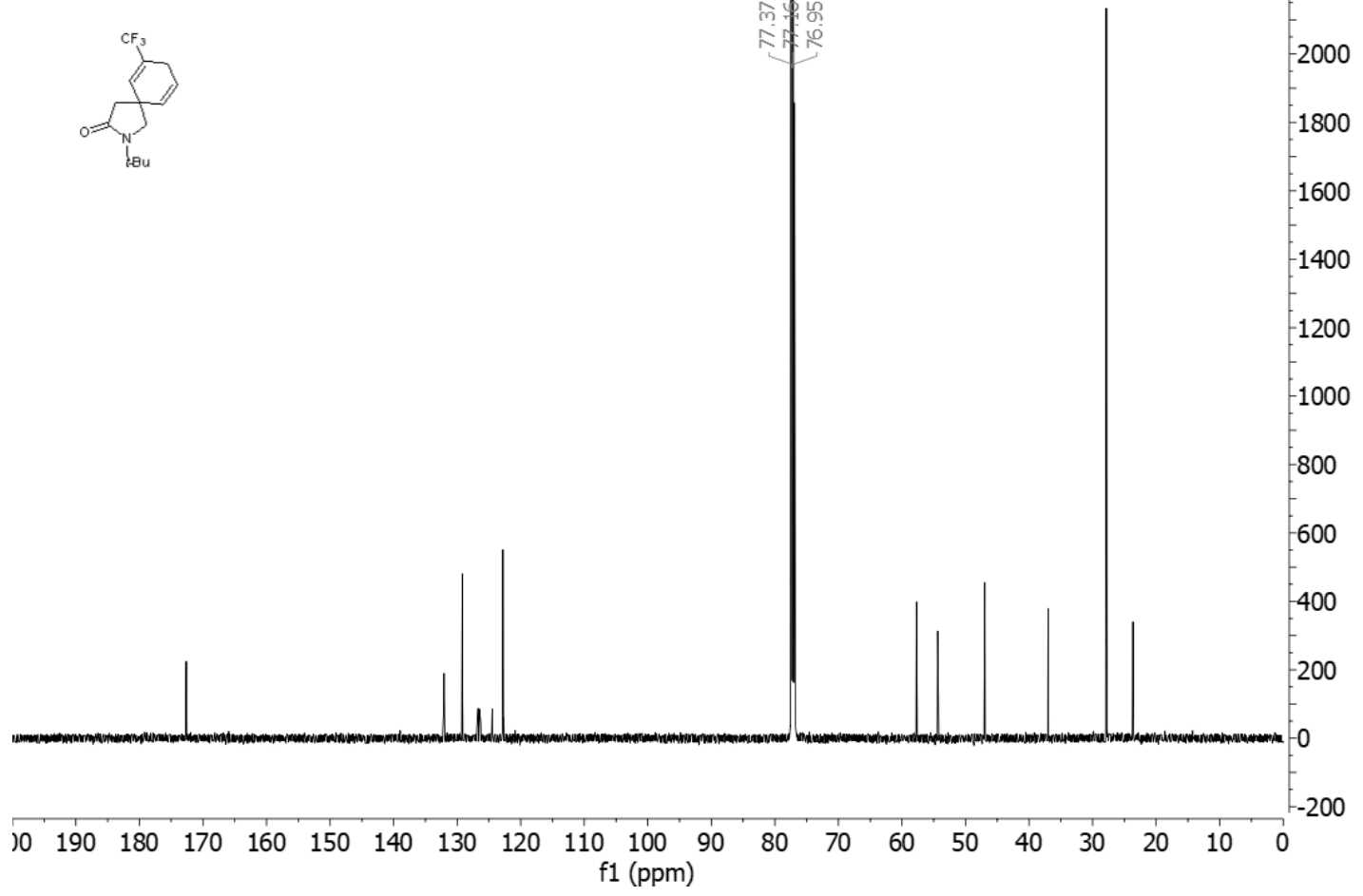


${ }^{1} \mathrm{H}$ NMR of 6

400.15 MHz

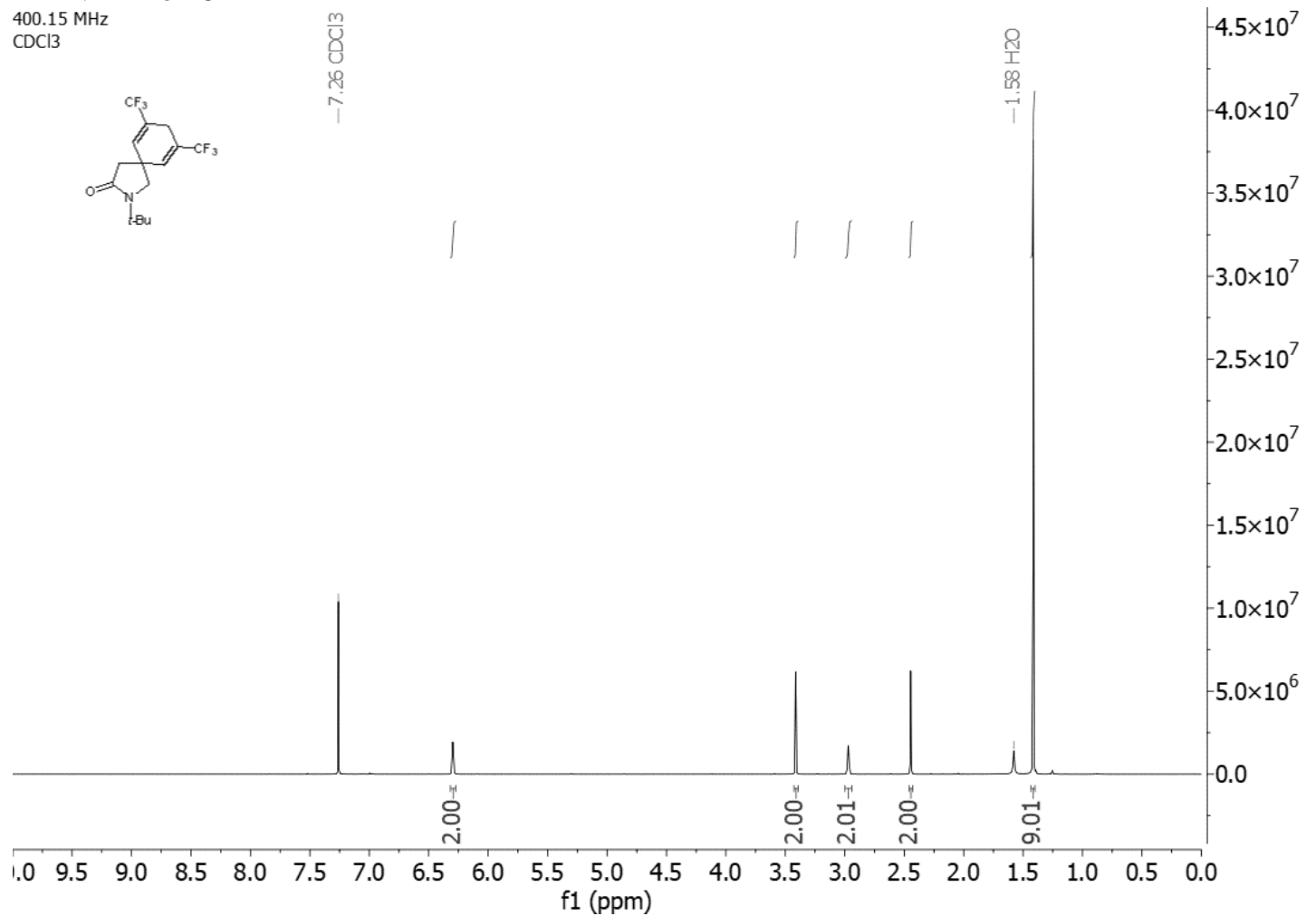

${ }^{13} \mathrm{C}$ NMR of 6

$150.93 \mathrm{MHz}$

$\mathrm{CDCl} 3$

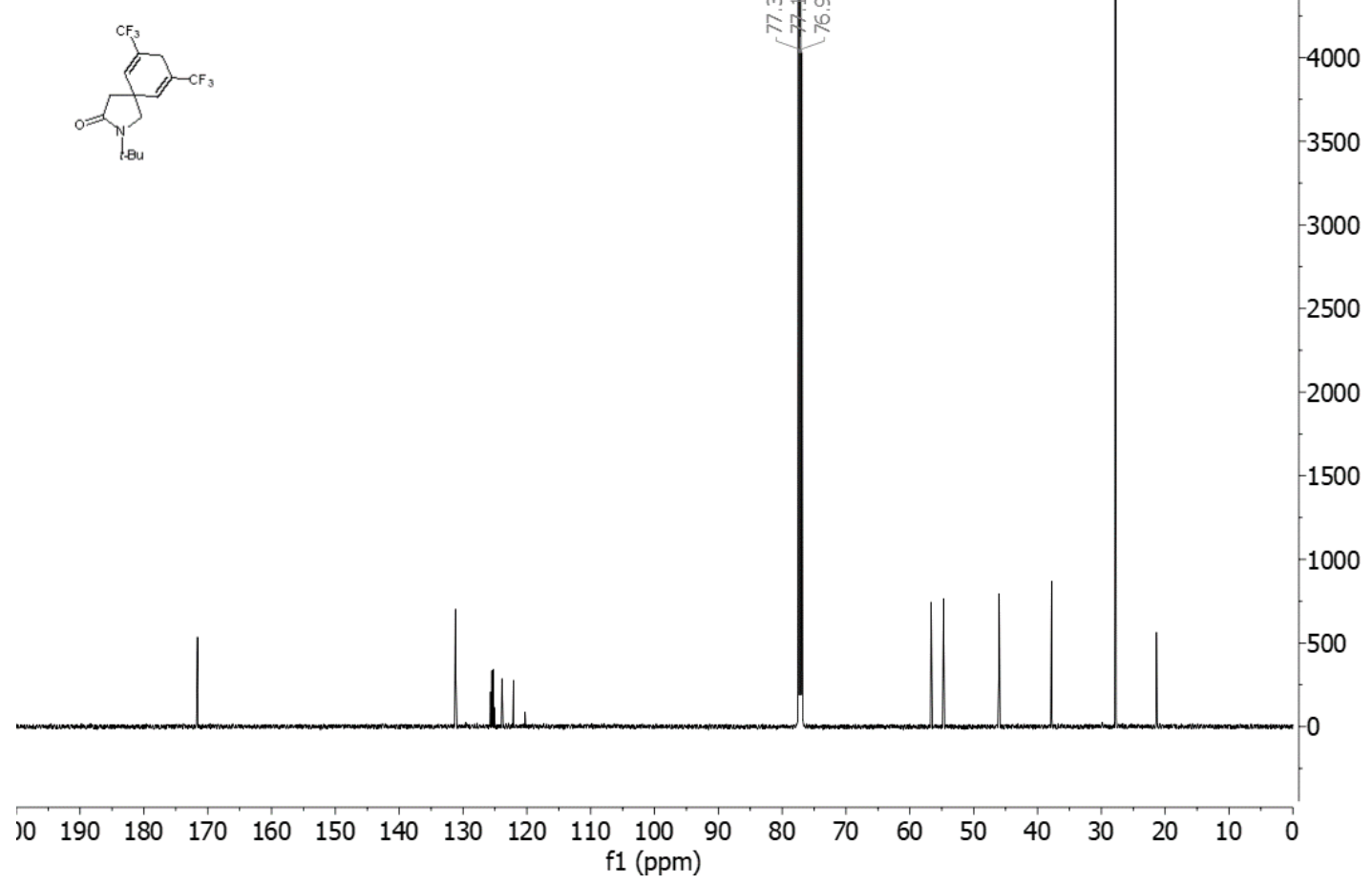


${ }^{1} \mathrm{H}$ NMR of 7

$600.18 \mathrm{MHz}$
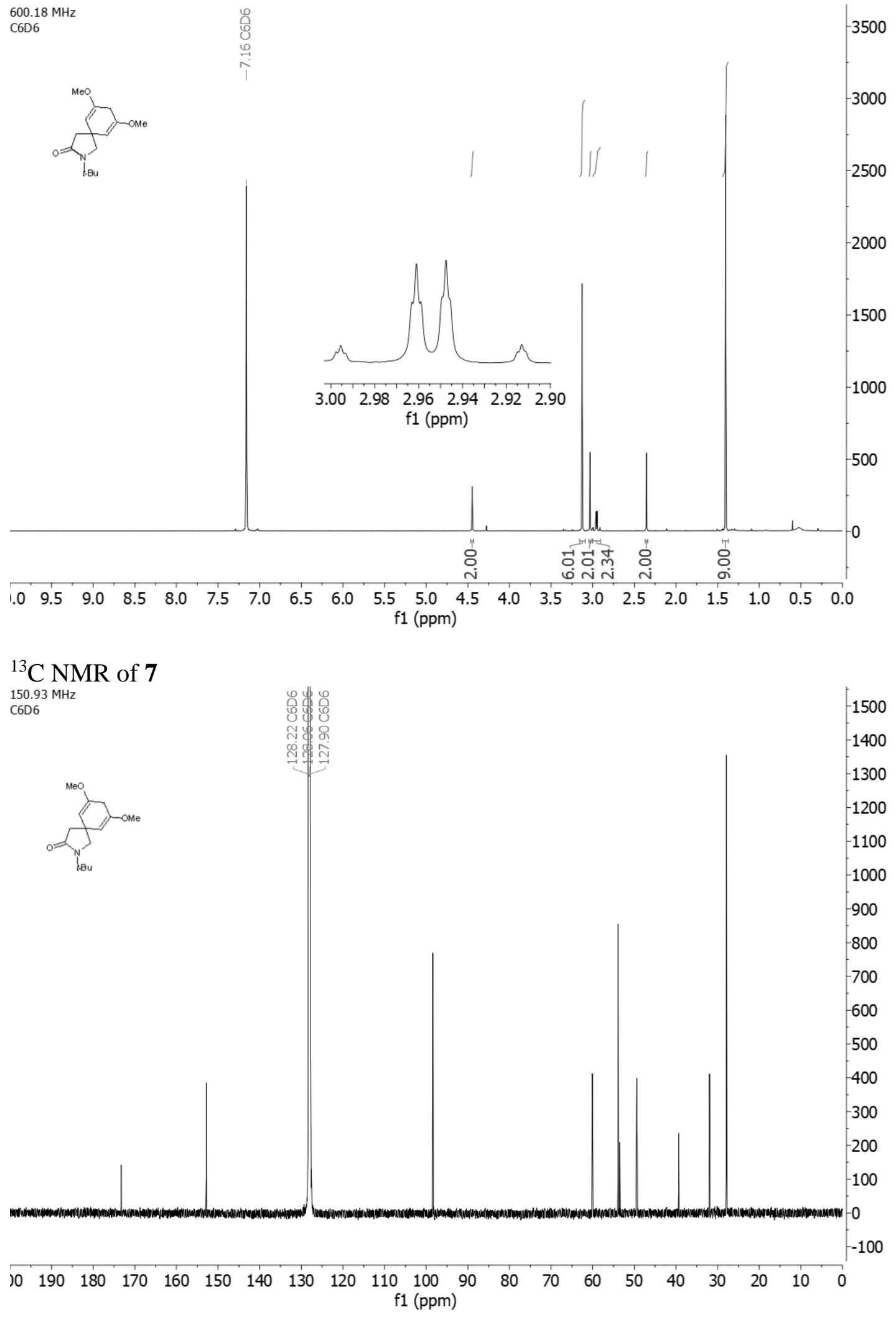
${ }^{1} \mathrm{H}$ NMR of 8

${ }_{\mathrm{CDCl}}^{600.18 \mathrm{MHz}}$

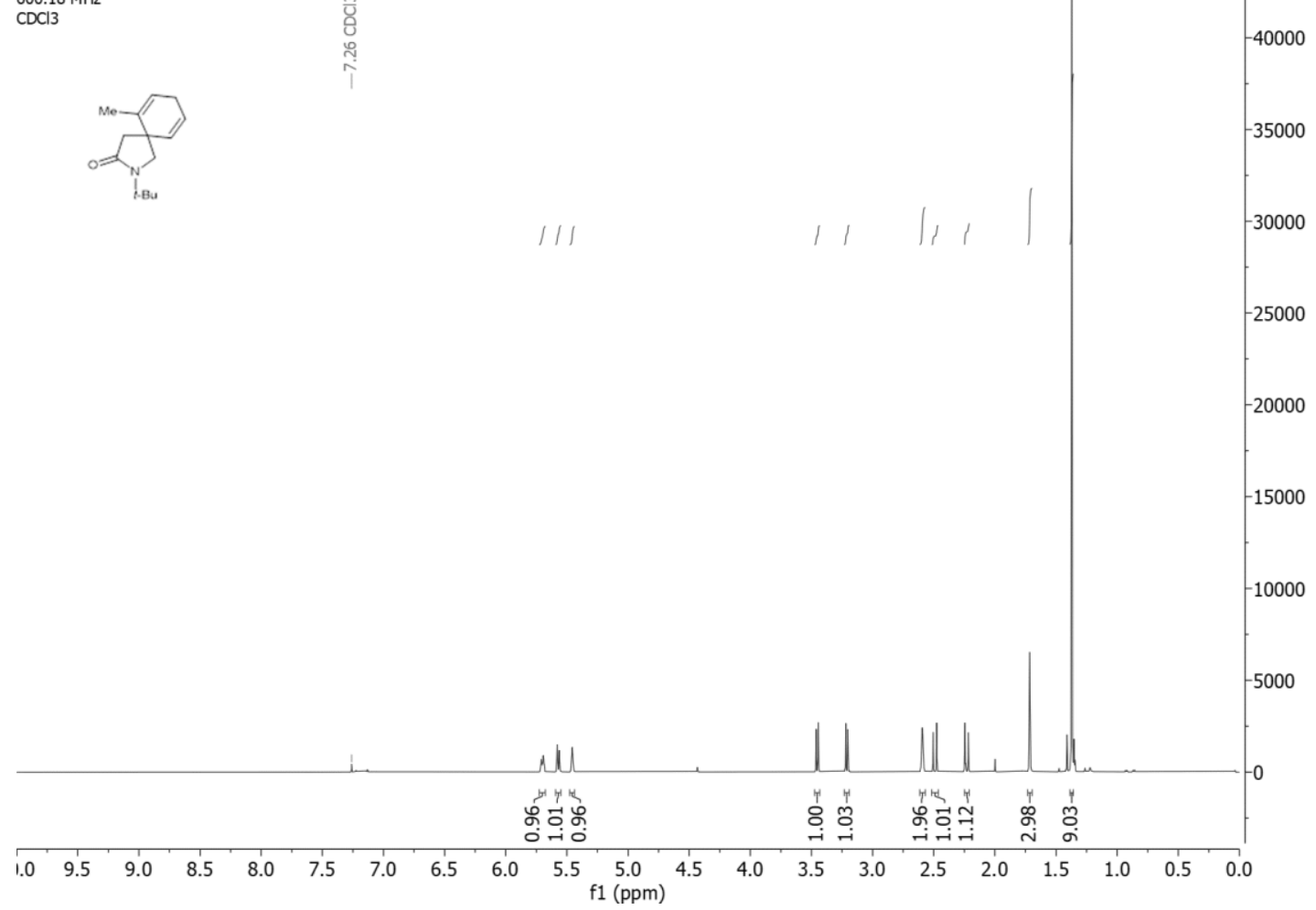

\section{${ }^{13} \mathrm{C}$ NMR of 8}

${ }_{150.93} \mathrm{MHz}$

$\mathrm{CDCl} 3$

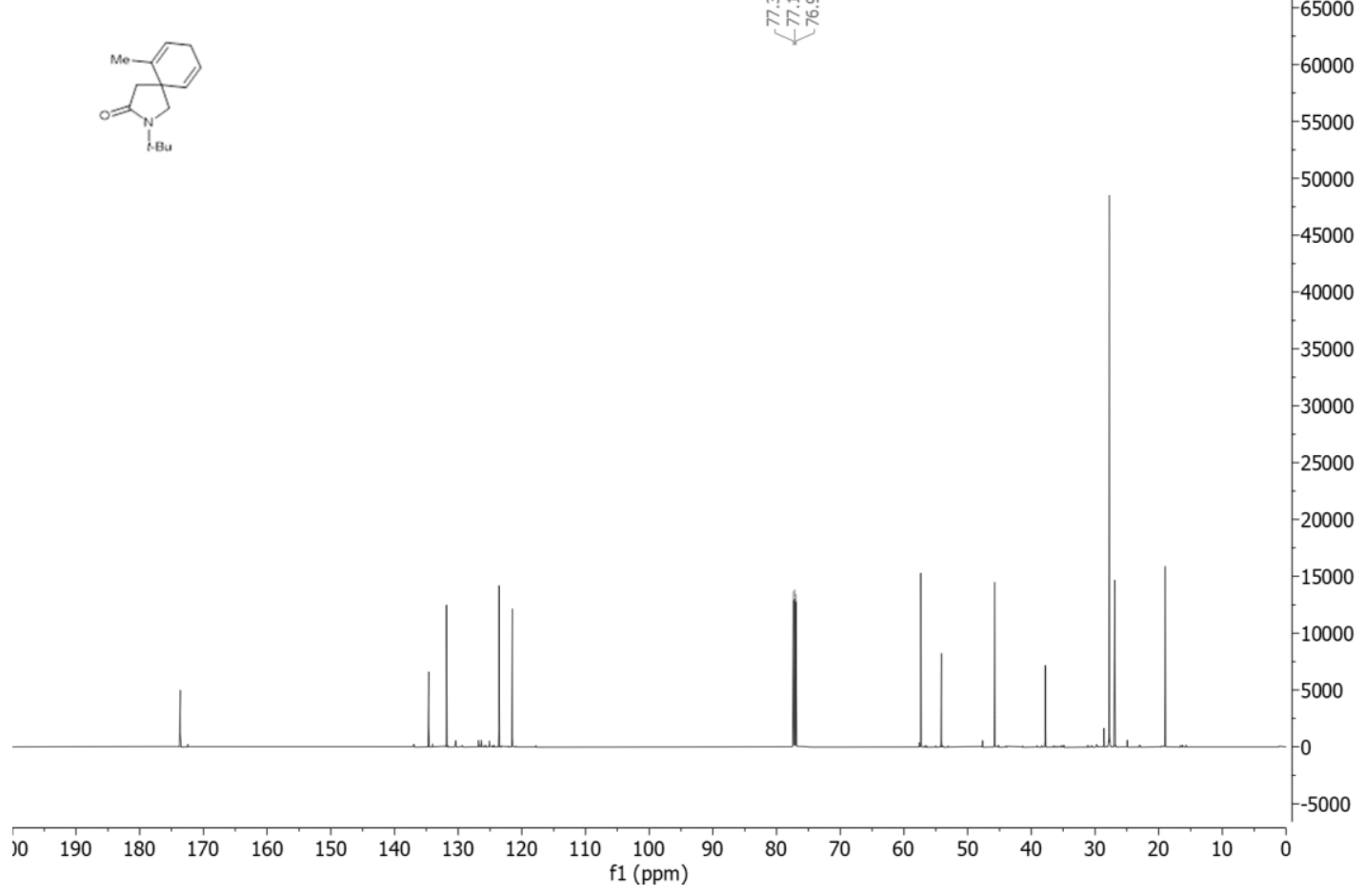




\section{${ }^{1} \mathrm{H}$ NMR of 9}

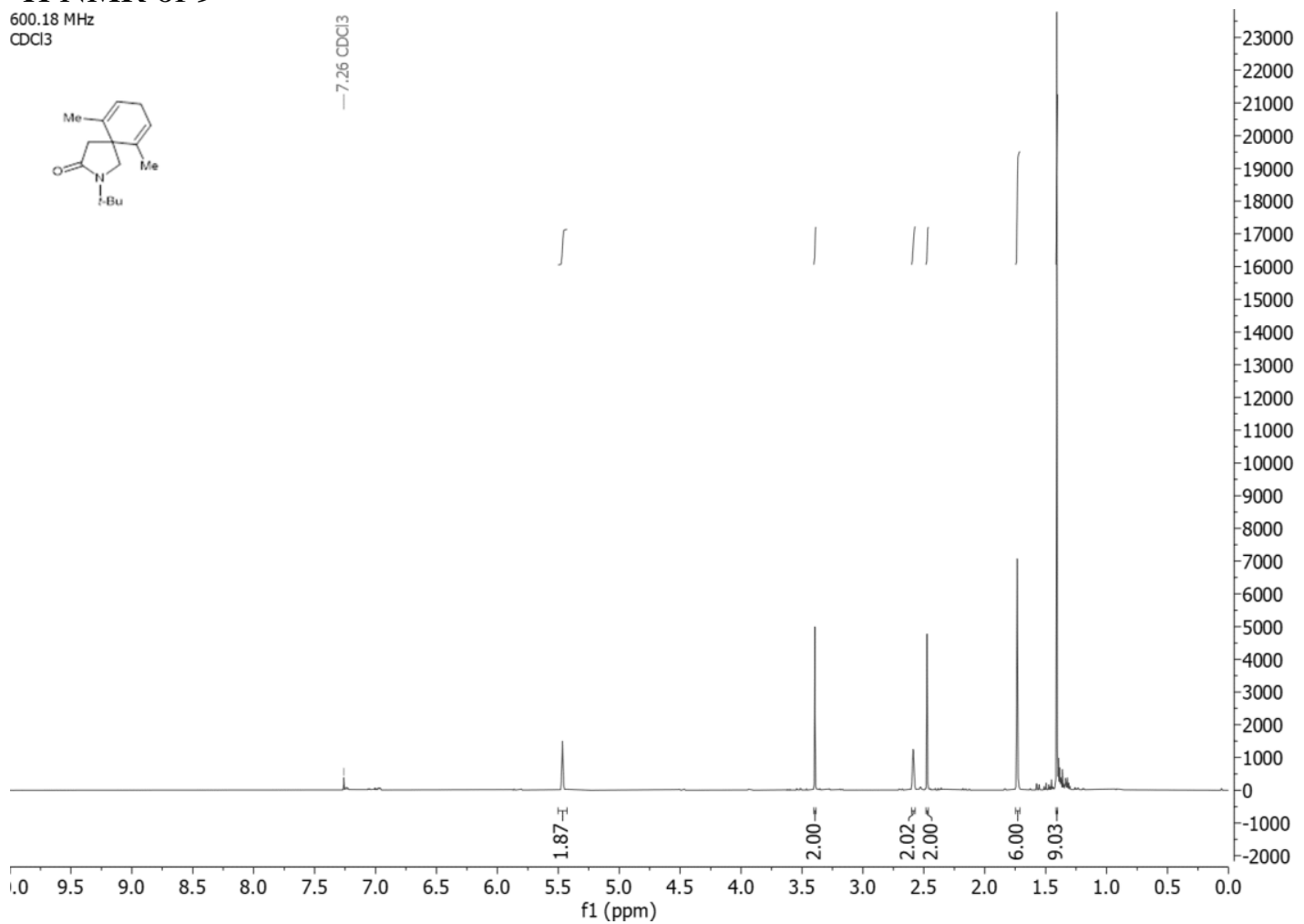

\section{${ }^{13} \mathrm{C}$ NMR of 9}

${ }_{150.93 \mathrm{MHz}}$

$\mathrm{CDCl} 3$
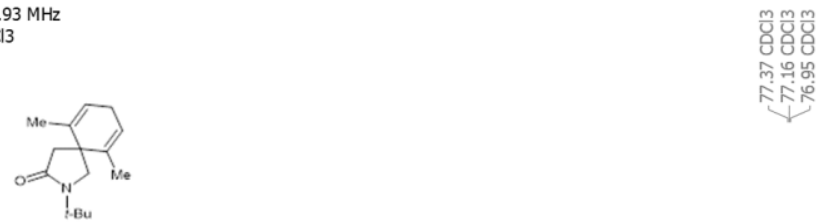

$-20000$

$-19000$

$-18000$

$-17000$

$-16000$

$-15000$

$-14000$

$-13000$

$-12000$

$-11000$

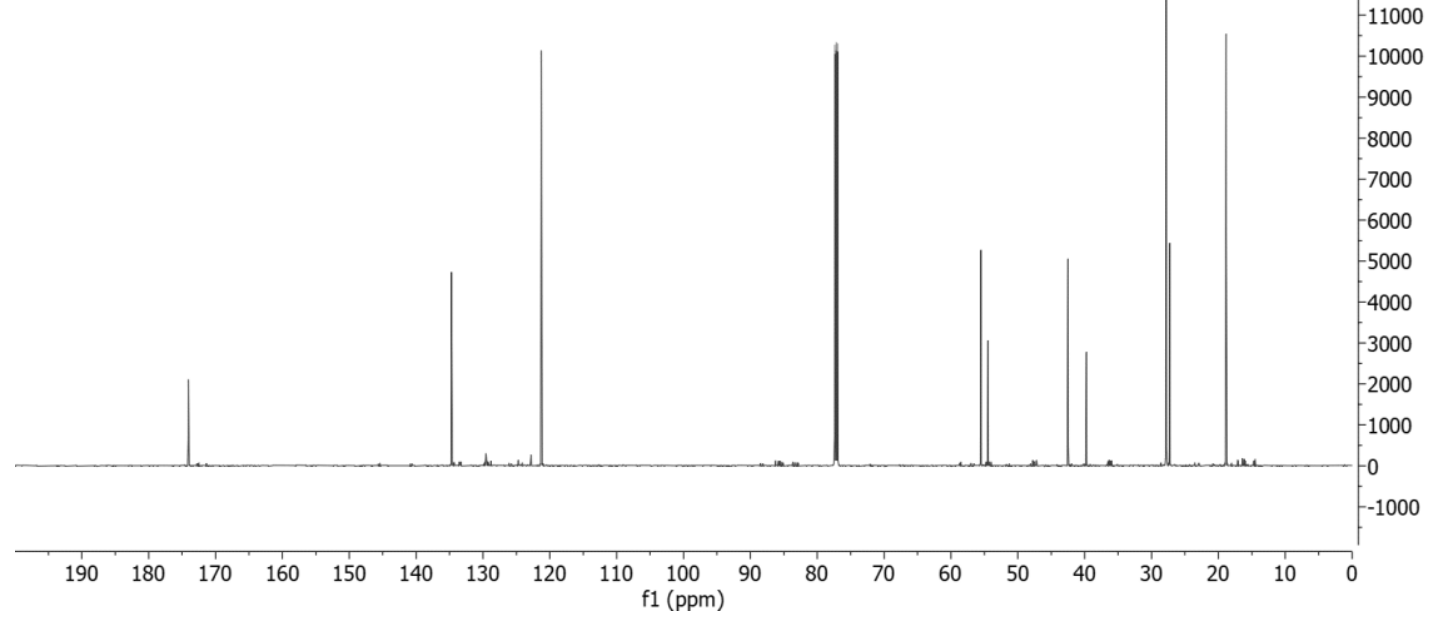




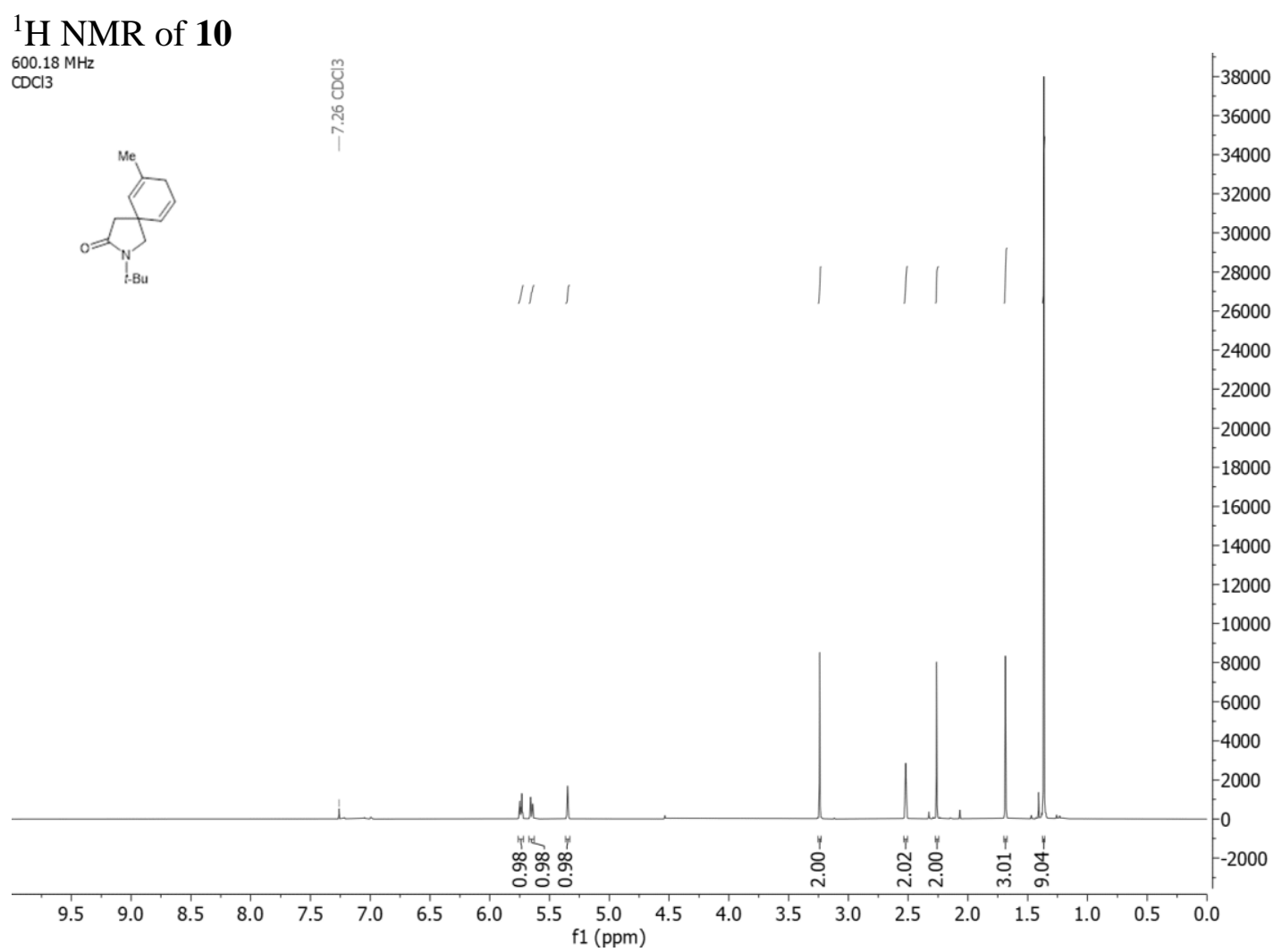

\section{${ }^{13} \mathrm{C}$ NMR of $\mathbf{1 0}$}

${ }_{150.93 \mathrm{MHz}}$

$\mathrm{CDCl} 3$

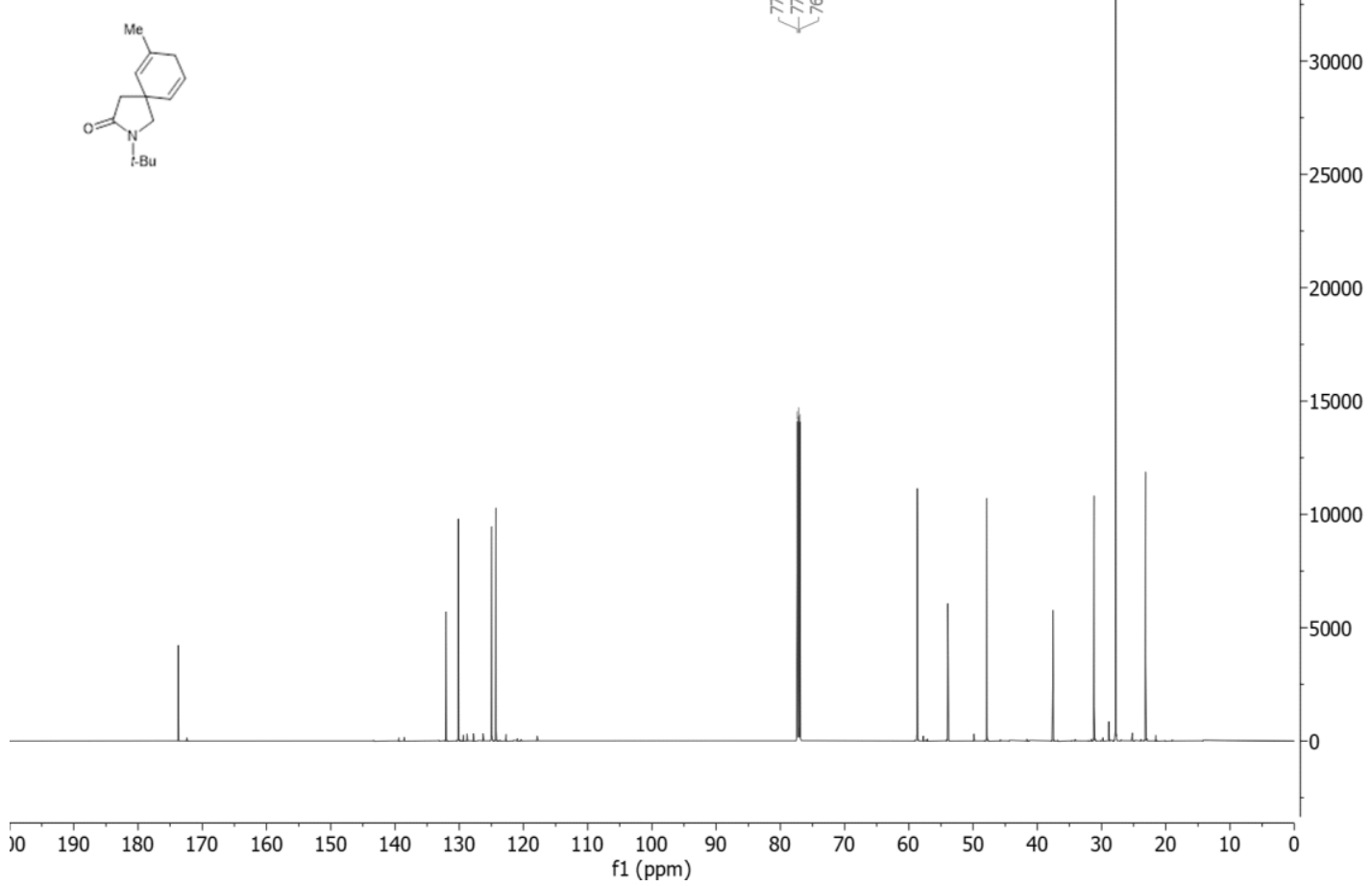


${ }^{1} \mathrm{H}$ NMR of $\mathbf{1 1}$

$400.15 \mathrm{MHz}$

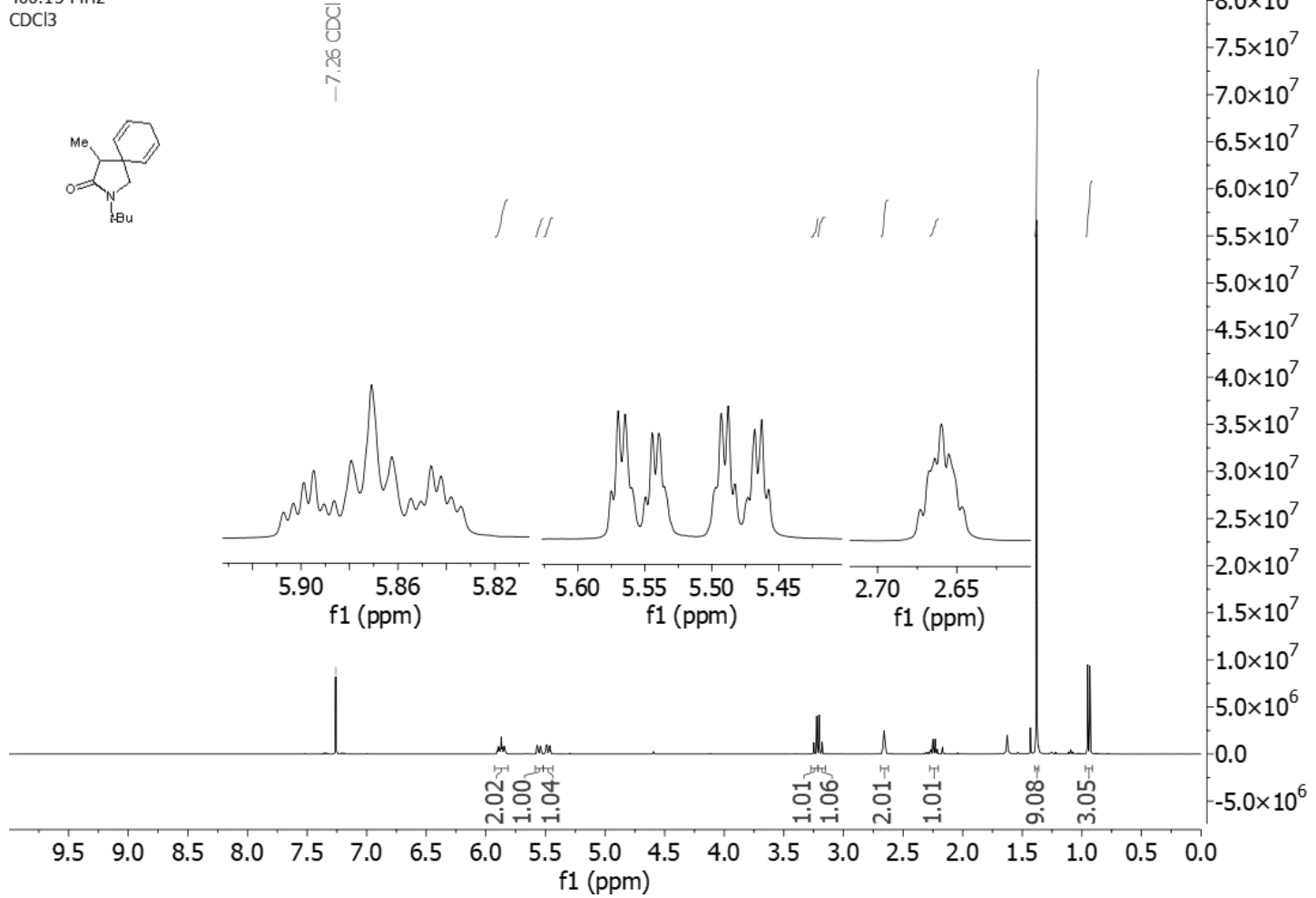

${ }^{13} \mathrm{C}$ NMR of $\mathbf{1 1}$

$150.93 \mathrm{MHz}$

CDCl3

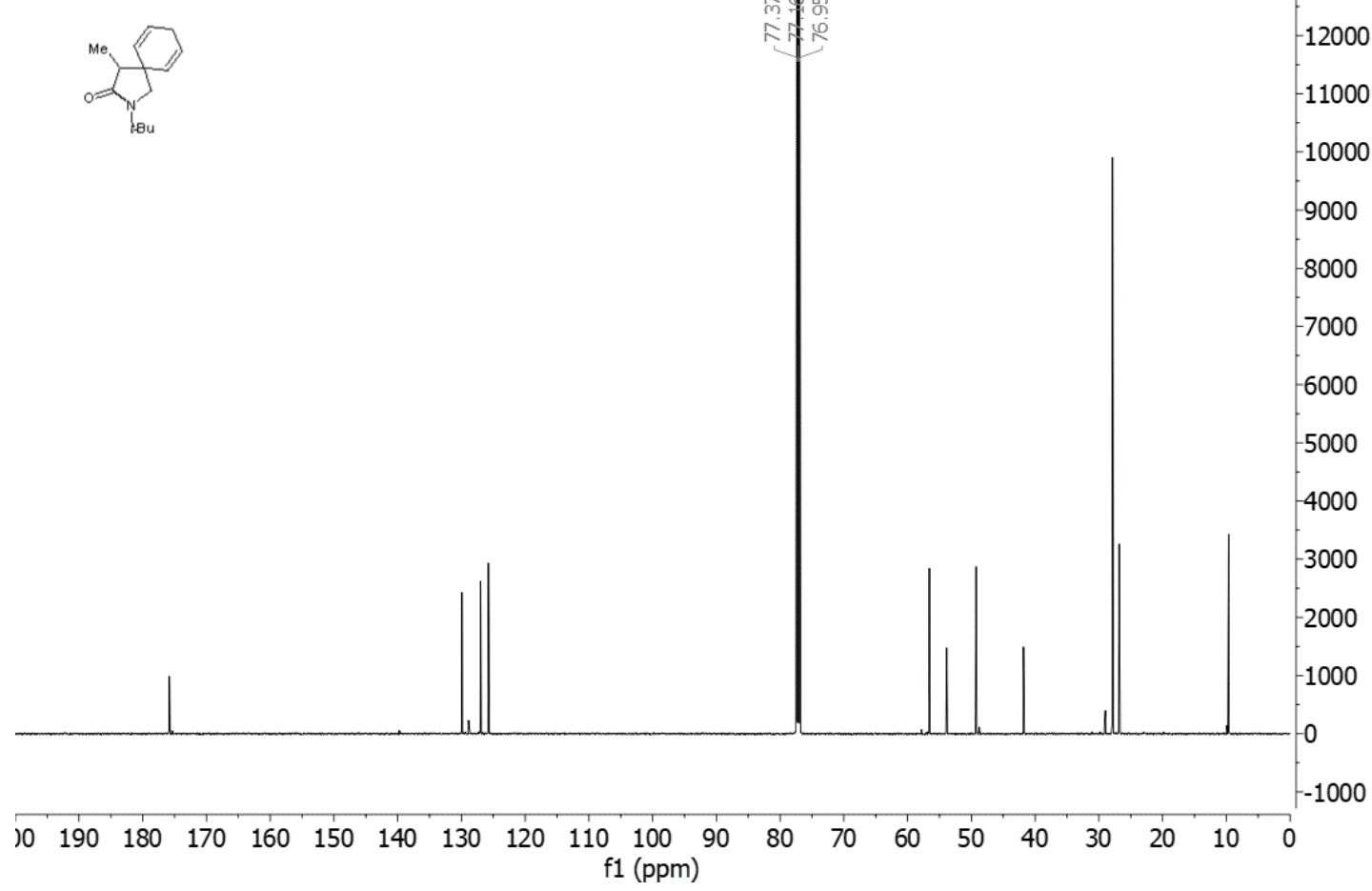


${ }^{1} \mathrm{H}$ NMR of 12

$400.15 \mathrm{MHz}$

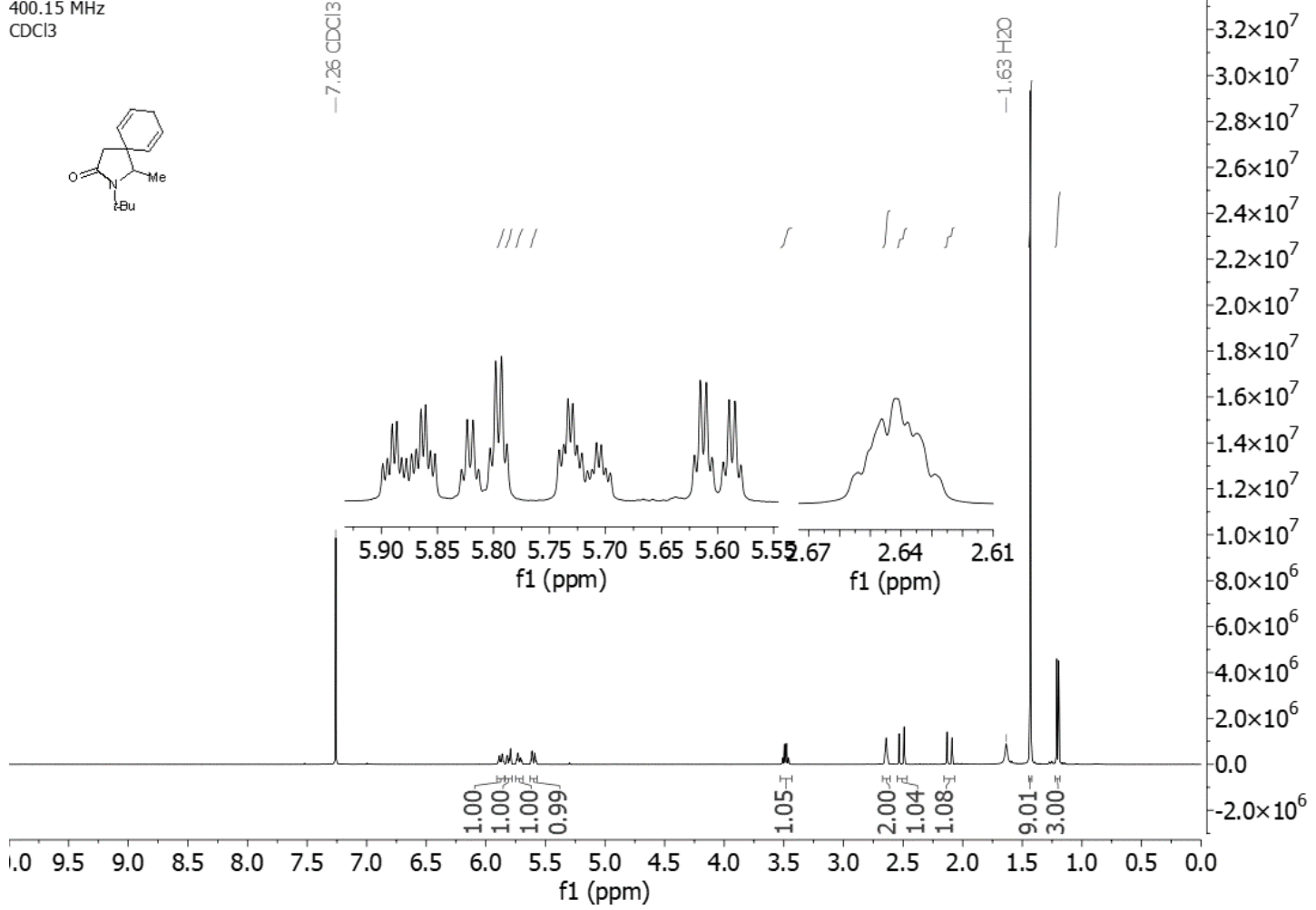

\section{${ }^{13} \mathrm{C}$ NMR of 12}

$150.93 \mathrm{MHz}$

$\mathrm{CDCl} 3$

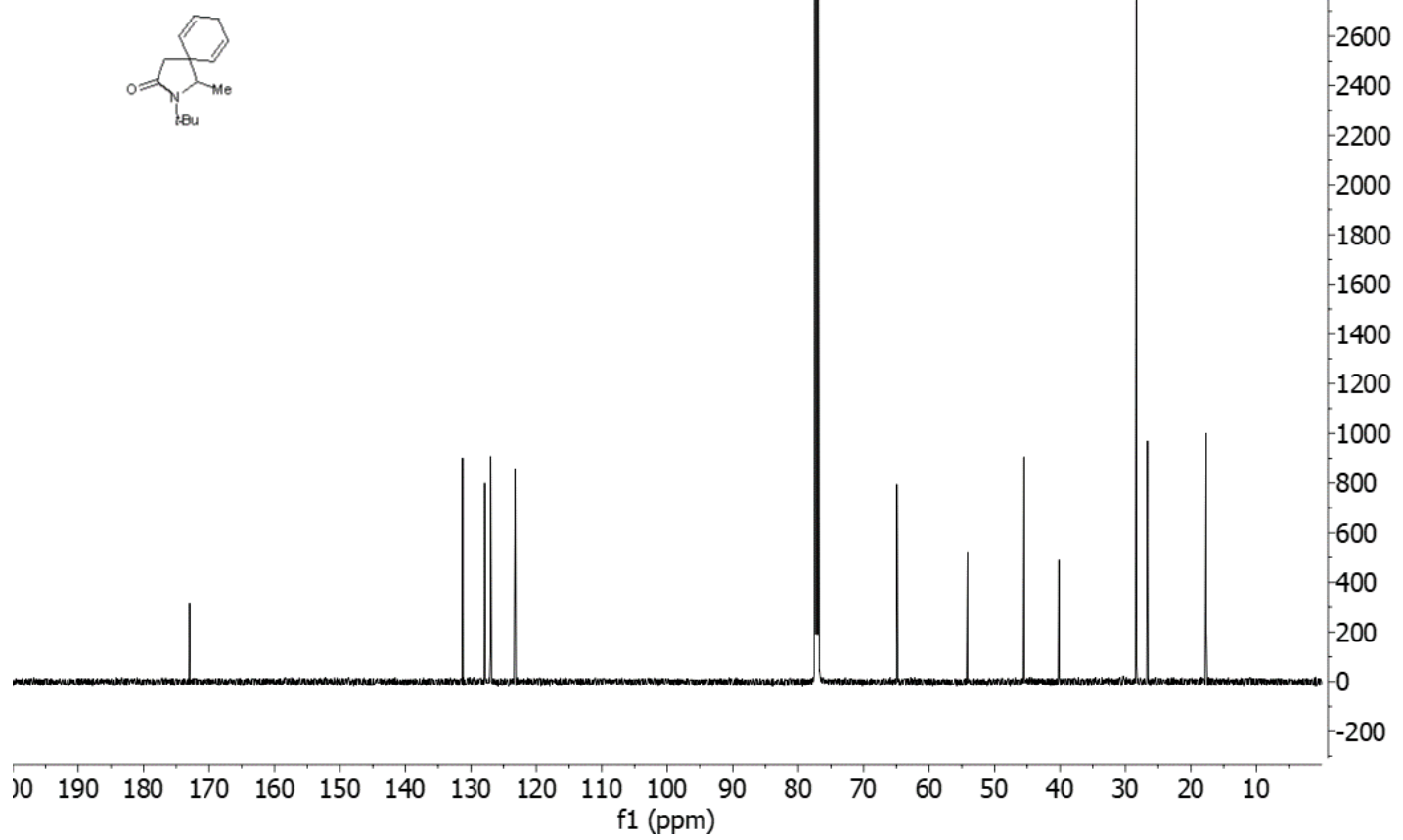


${ }^{1} \mathrm{H}$ NMR of 13

${ }_{600.18}^{60 \mathrm{MHz}}$

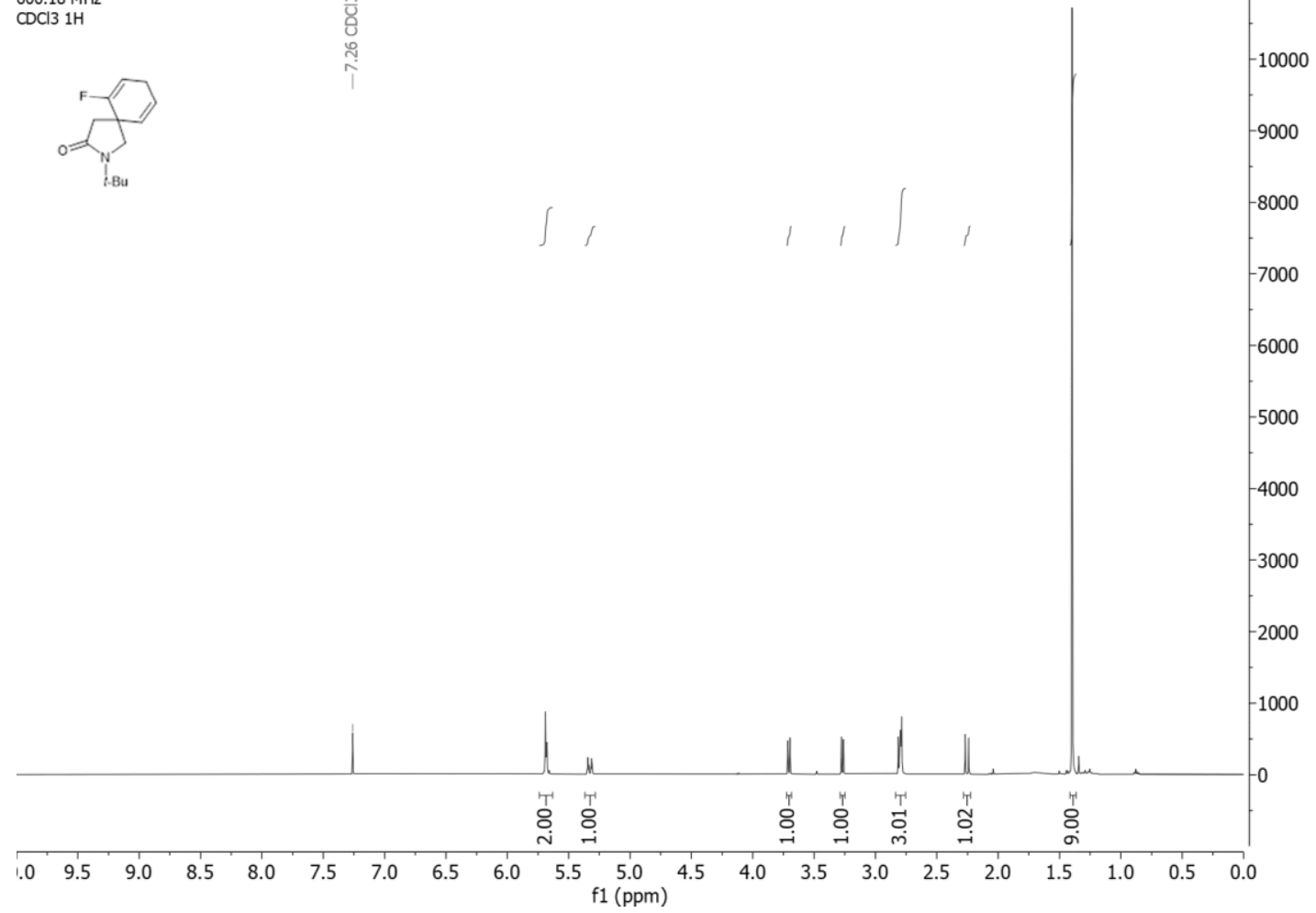

\section{${ }^{13} \mathrm{C}$ NMR of 13}

$150.93 \mathrm{MHz}$

$\mathrm{CDCl} 313 \mathrm{C}$

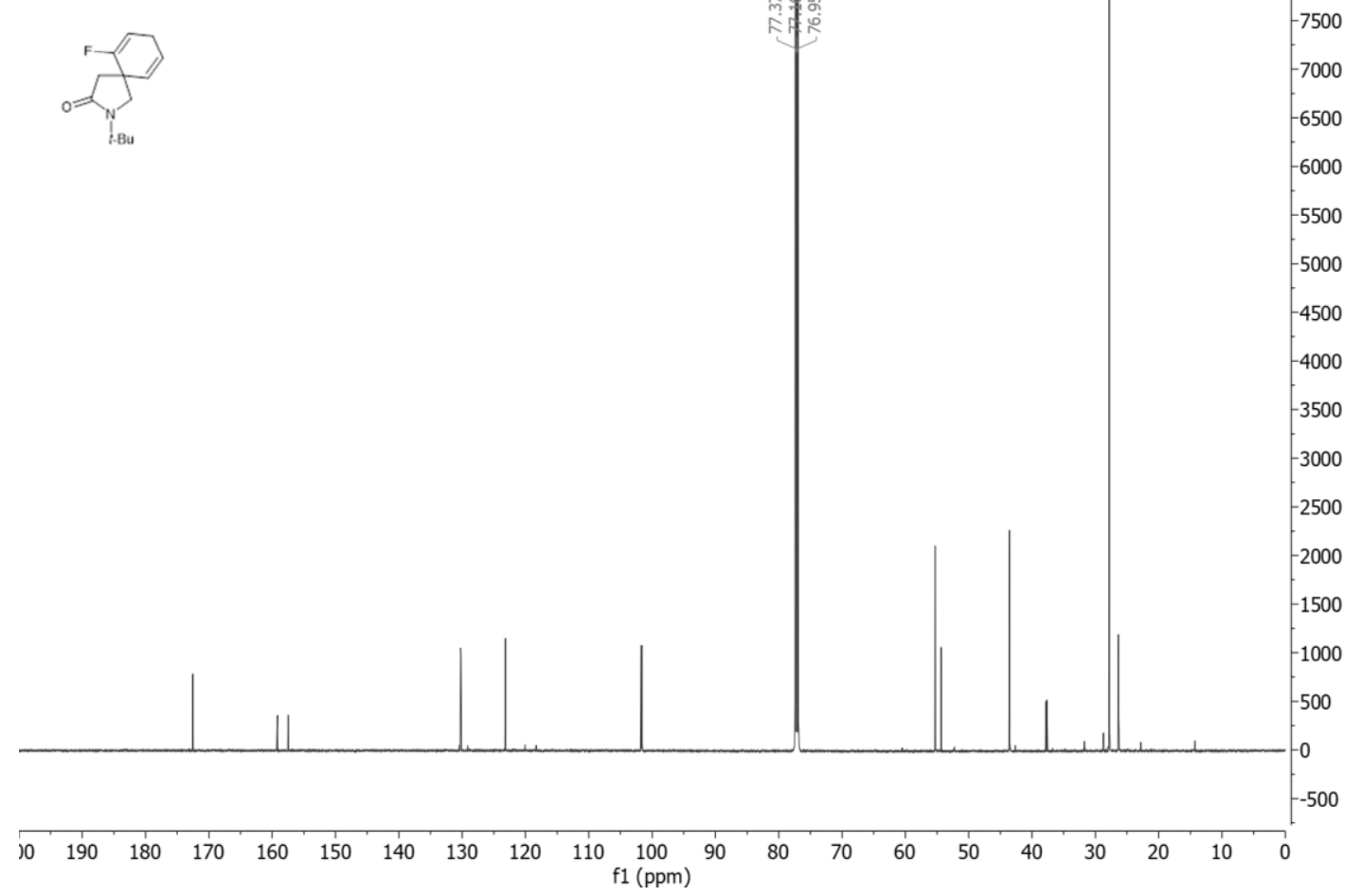


${ }^{1} \mathrm{H}$ NMR of 14

$600.18 \mathrm{MHz}$

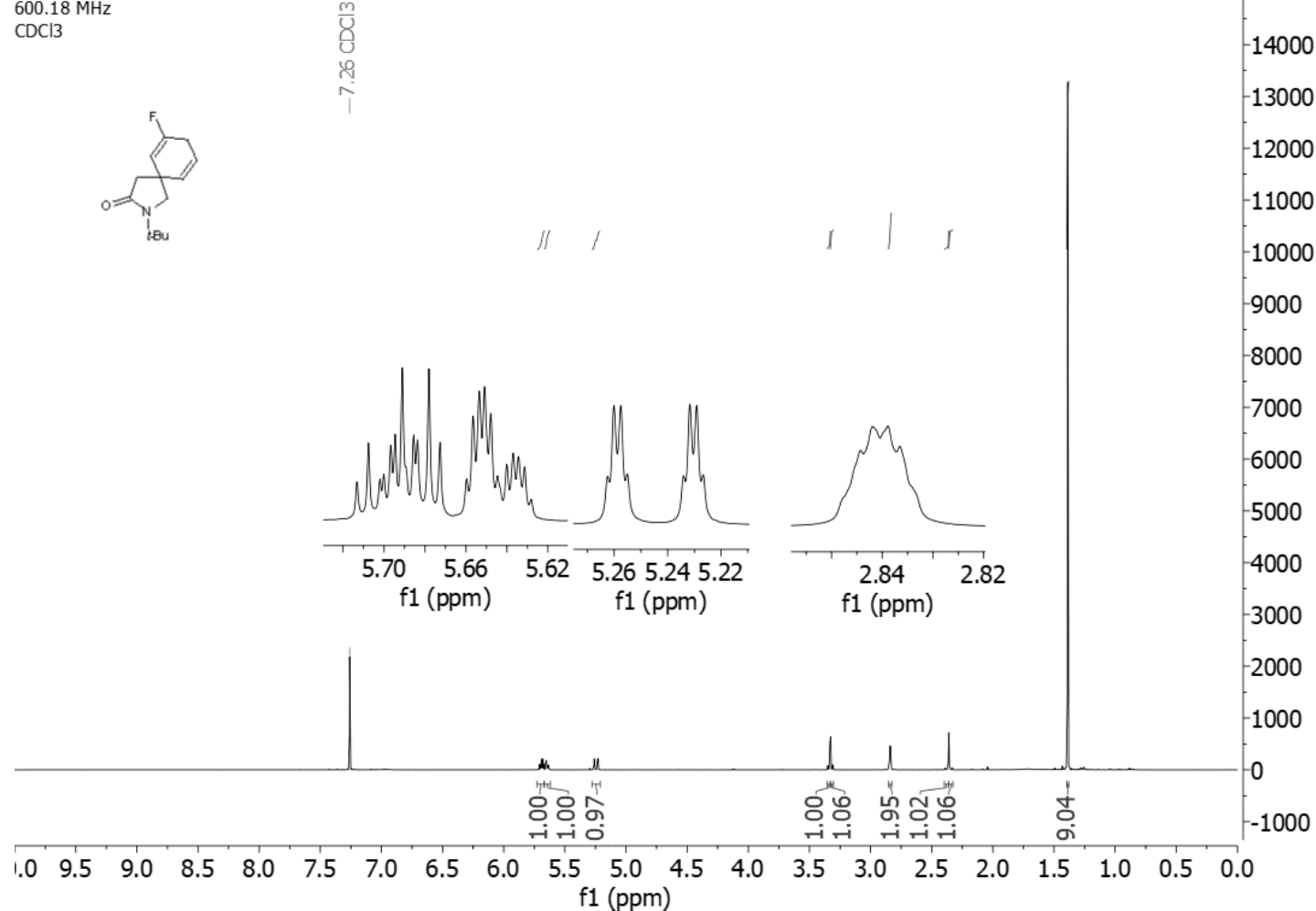

\section{${ }^{13} \mathrm{C}$ NMR of 14}

$150.93 \mathrm{MHz}$

$\mathrm{CDCl} 3$

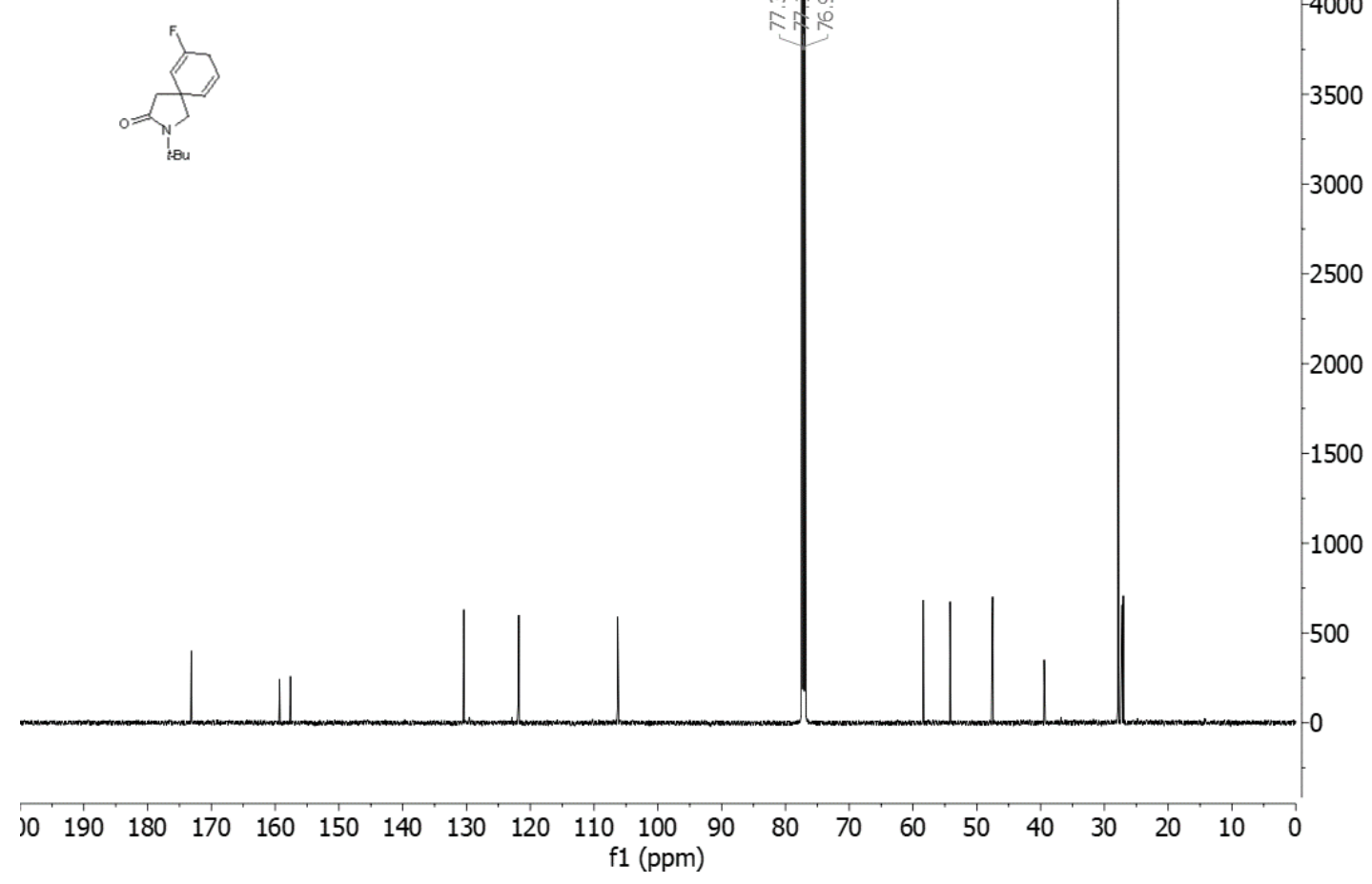




\section{${ }^{1} \mathrm{H}$ NMR of $\mathbf{1 5}$}

$600.18 \mathrm{MHz}$
$\mathrm{CDCl} 3 \mathrm{H} 1$

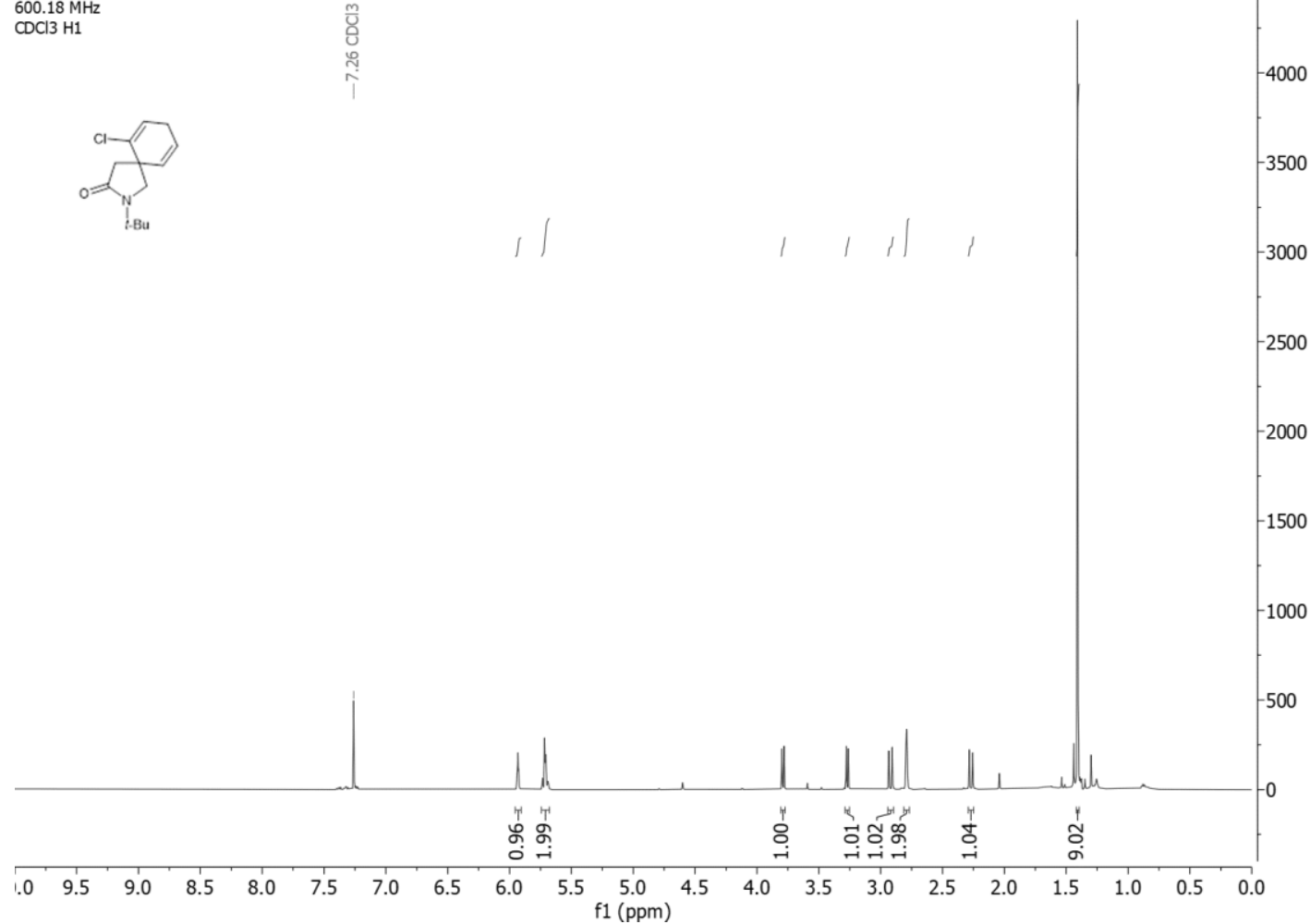

\section{${ }^{13} \mathrm{C}$ NMR of $\mathbf{1 5}$}

$150.93 \mathrm{MHz}$

CDCl3 $\mathrm{C} 13$

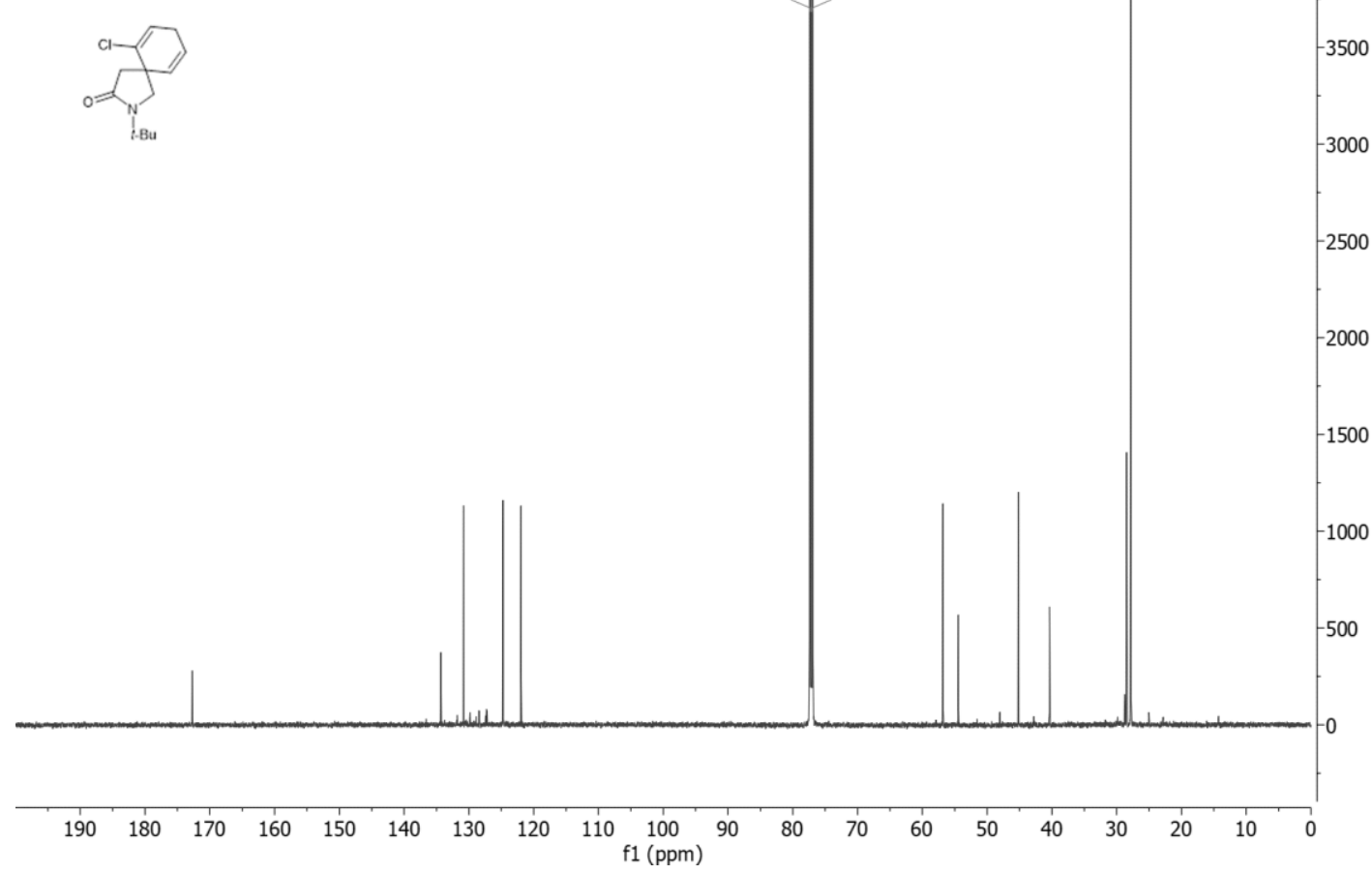


${ }^{1} \mathrm{H}$ NMR of 16

$600.18 \mathrm{MHz}$
$0.0131 \mathrm{H}$

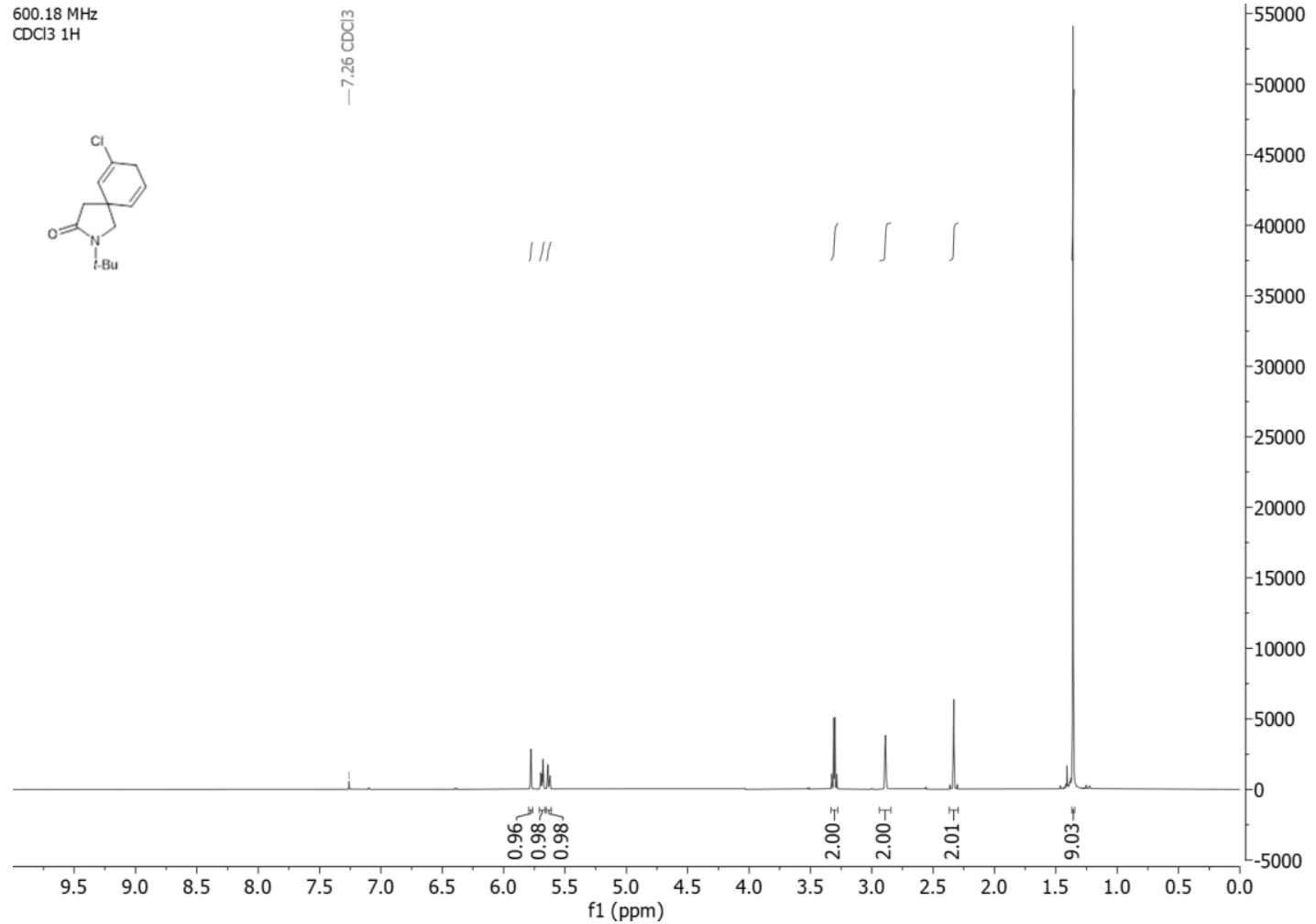

${ }^{13} \mathrm{C}$ NMR of 16

$150.93 \mathrm{MHz}$

CDCl3 $13 \mathrm{C}$
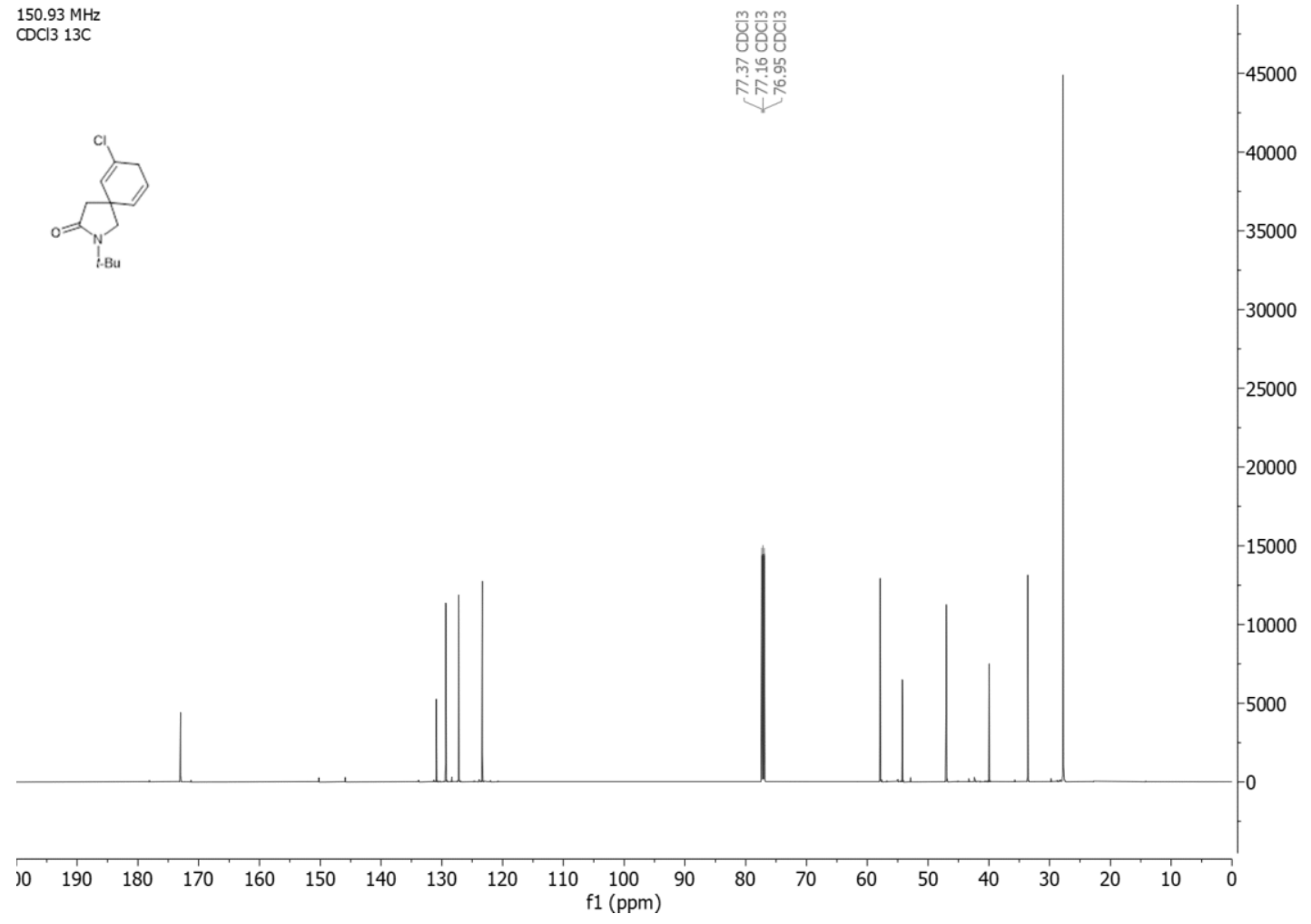
${ }^{1} \mathrm{H}$ NMR of $\mathbf{1 7}$ diastereomer $\mathbf{1}$

$400.15 \mathrm{MHz}$

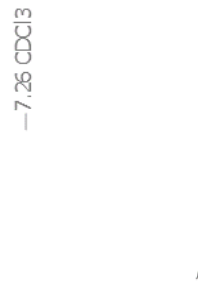

$2.8 \times 10^{7}$
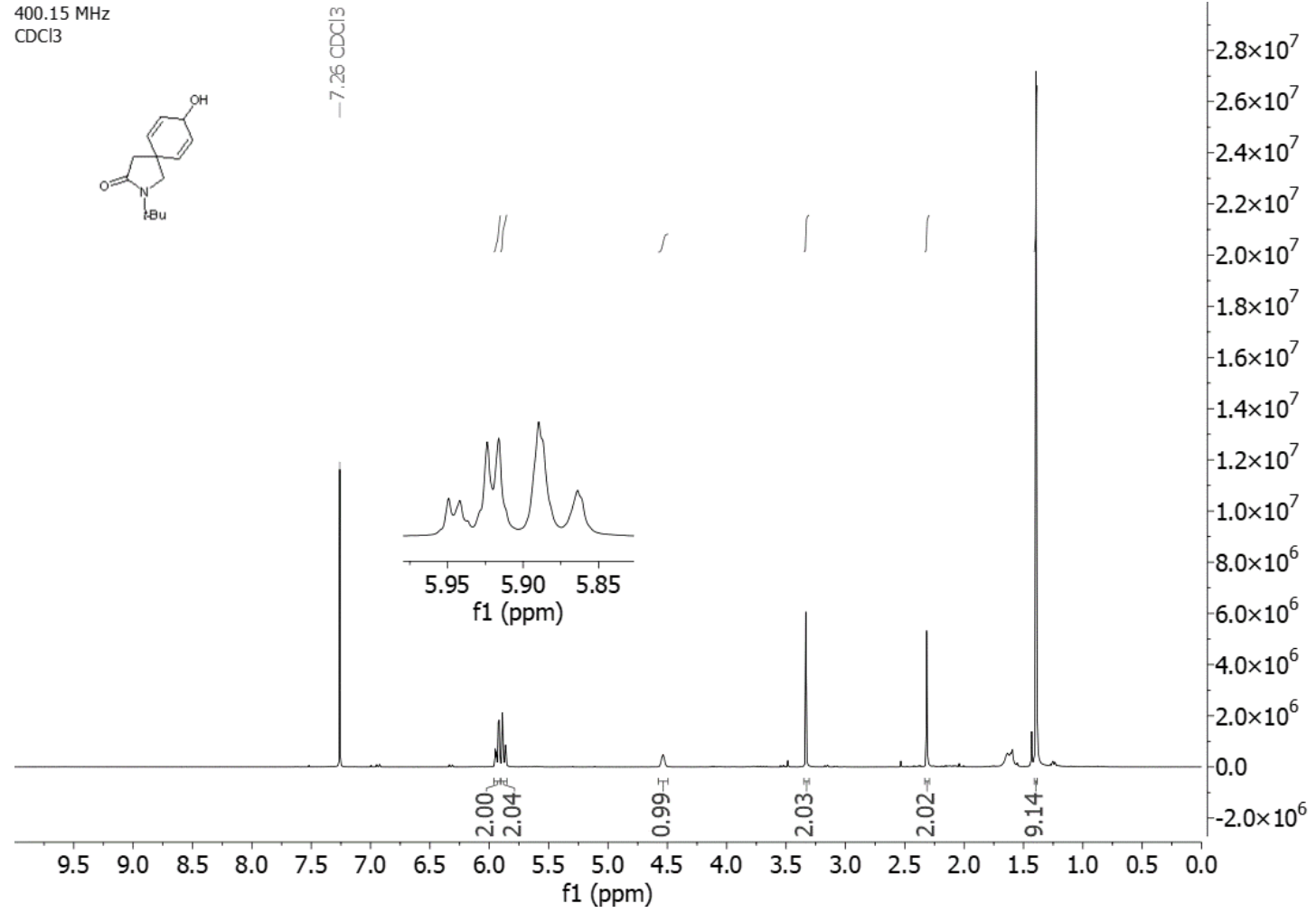

${ }^{13} \mathrm{C}$ NMR of 17 diastereomer 1 $100.63 \mathrm{MHz}$ $\mathrm{CDCl} 3$

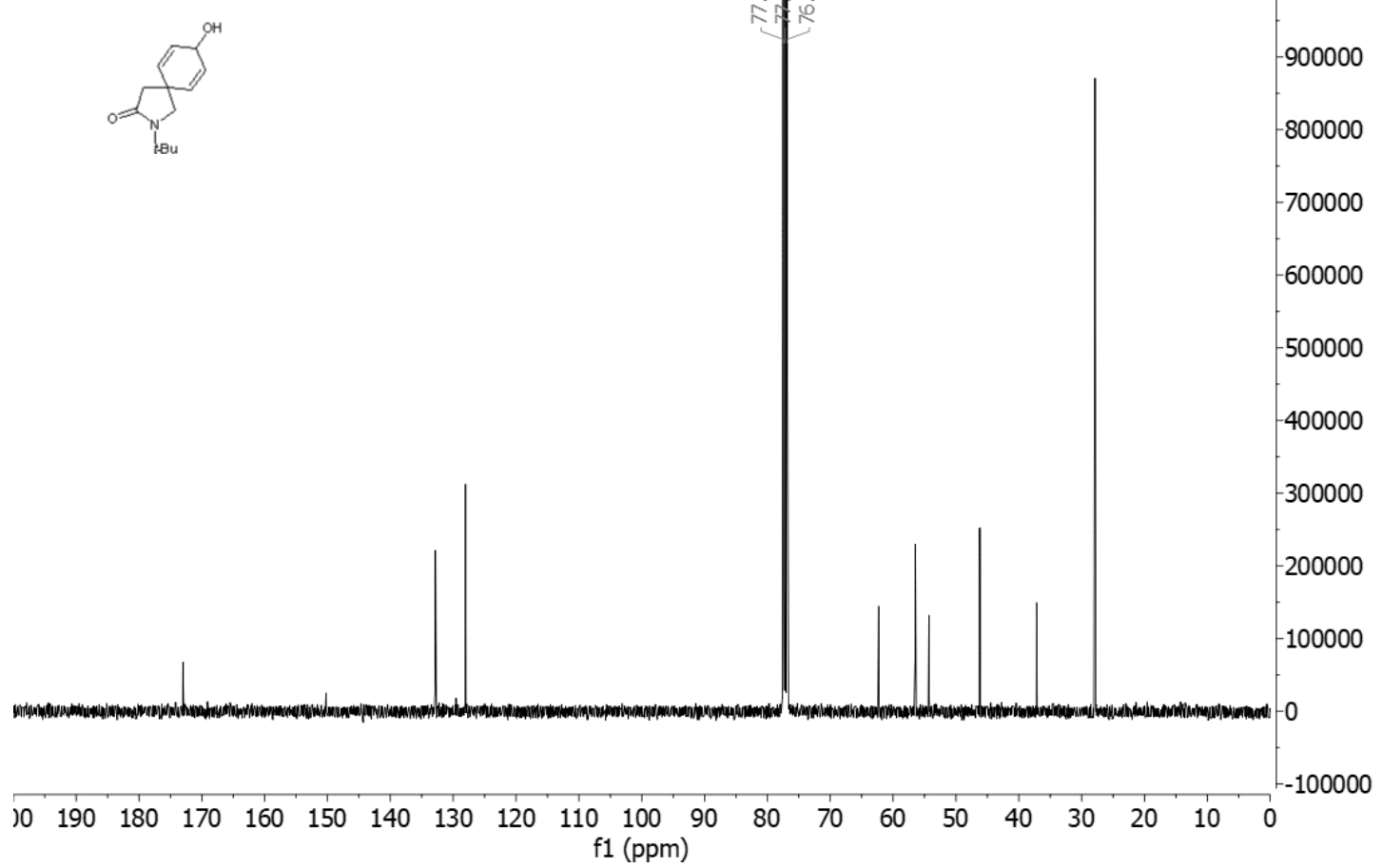


${ }^{1} \mathrm{H}$ NMR of $\mathbf{1 7}$ diastereomer 2

$400.15 \mathrm{MHz}$
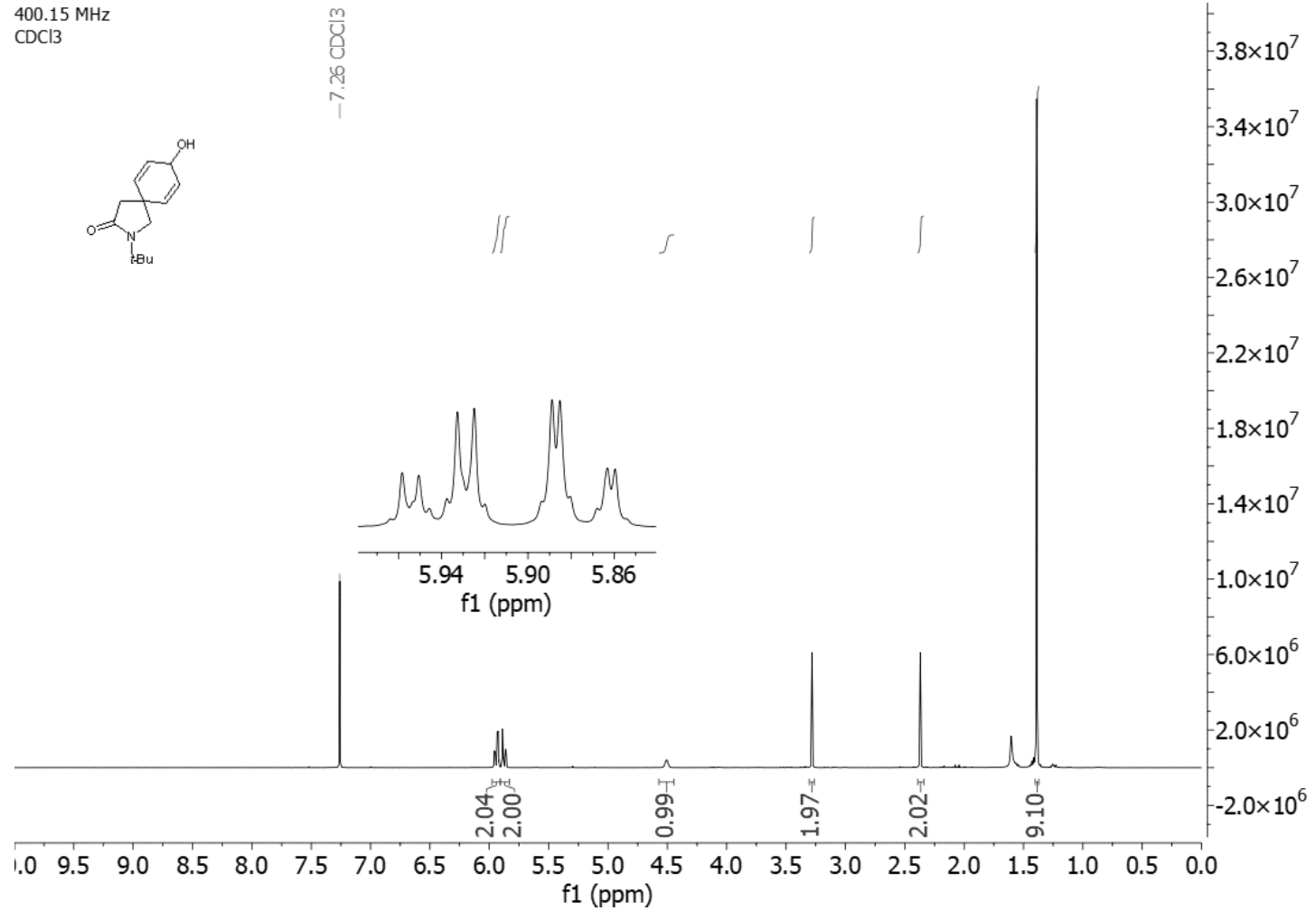

${ }^{13} \mathrm{C}$ NMR of 17 diastereomer 2 $100.63 \mathrm{MHz}$ $\mathrm{CDCl} 3$

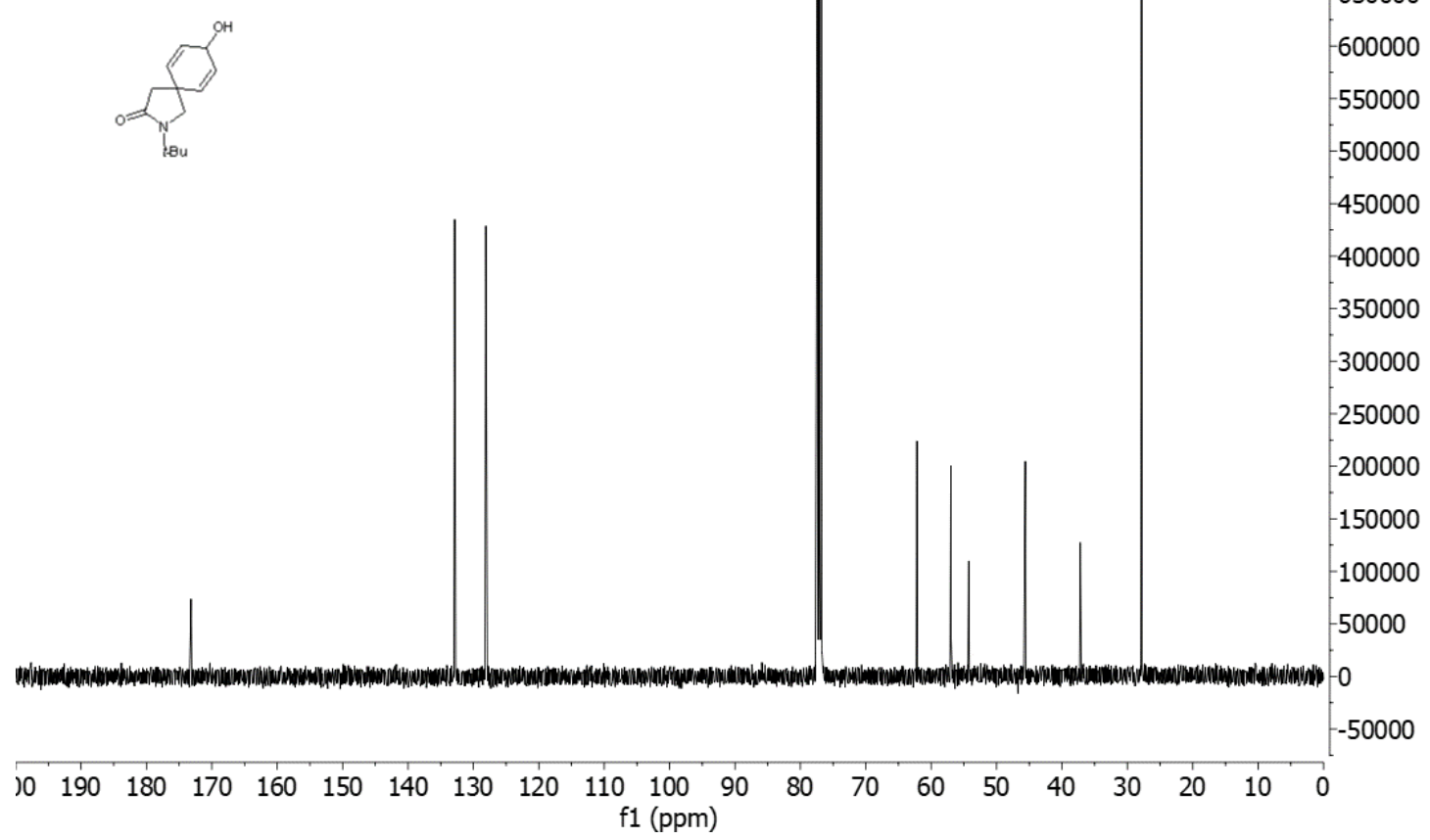
1 (ppm) 
${ }^{1} \mathrm{H}$ NMR of $\mathbf{1 8}$

${ }_{0600}^{400.15} \mathrm{MHz}$

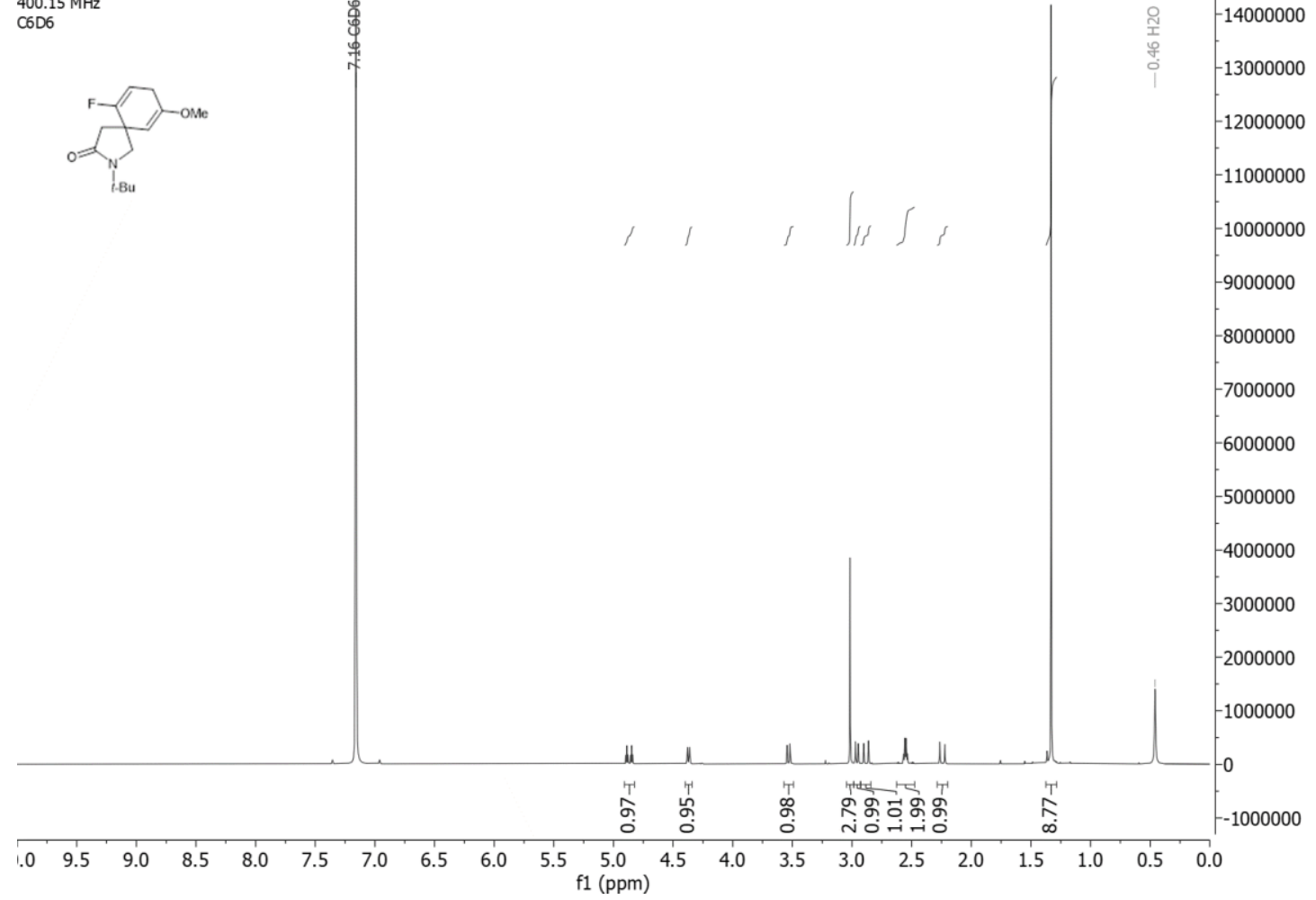

${ }^{13} \mathrm{C}$ NMR of $\mathbf{1 8}$

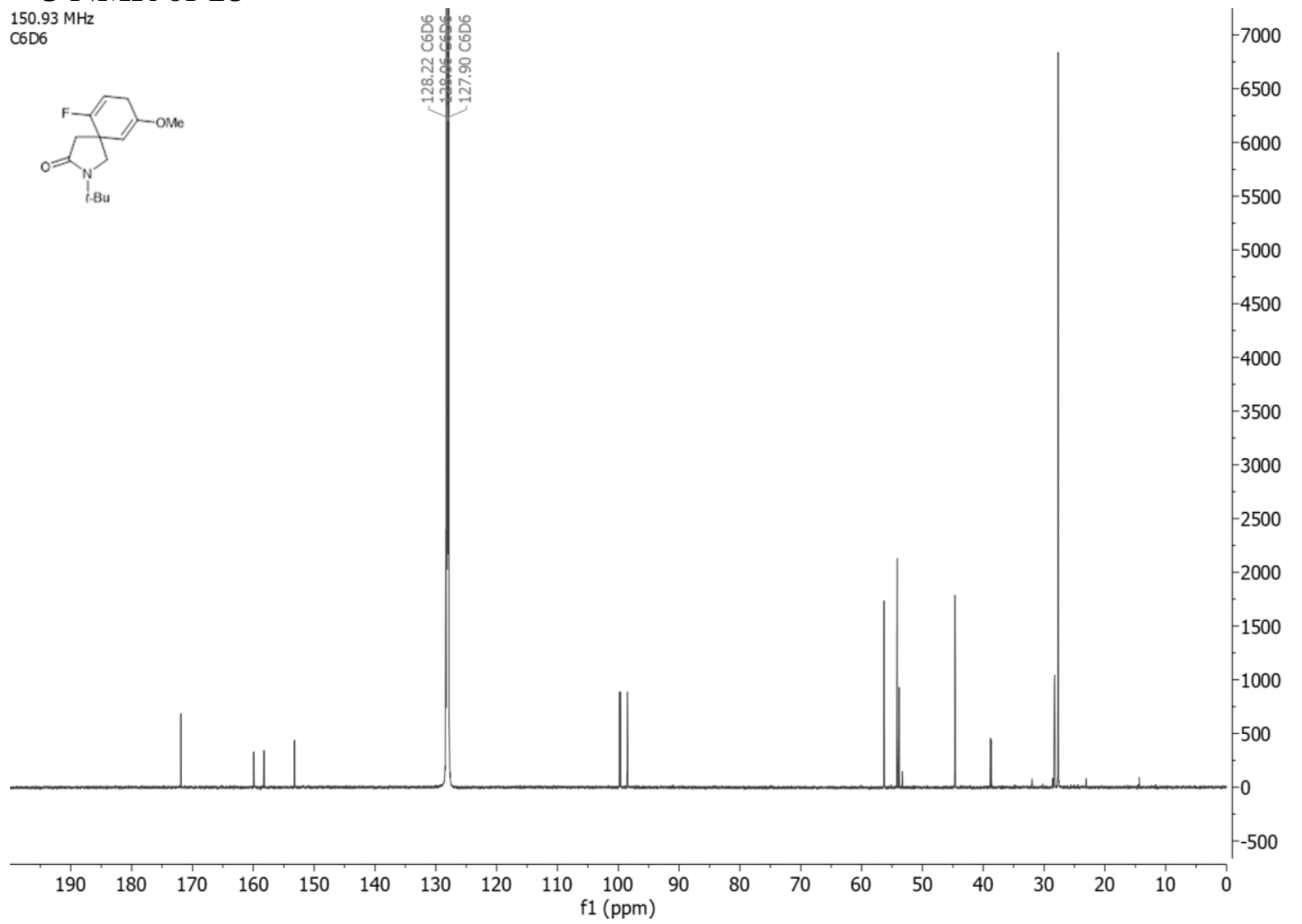


${ }^{1} \mathrm{H}$ NMR of 19

${ }_{0 D C D}^{600.18} \mathrm{MHz}$

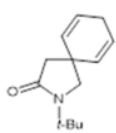

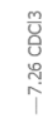

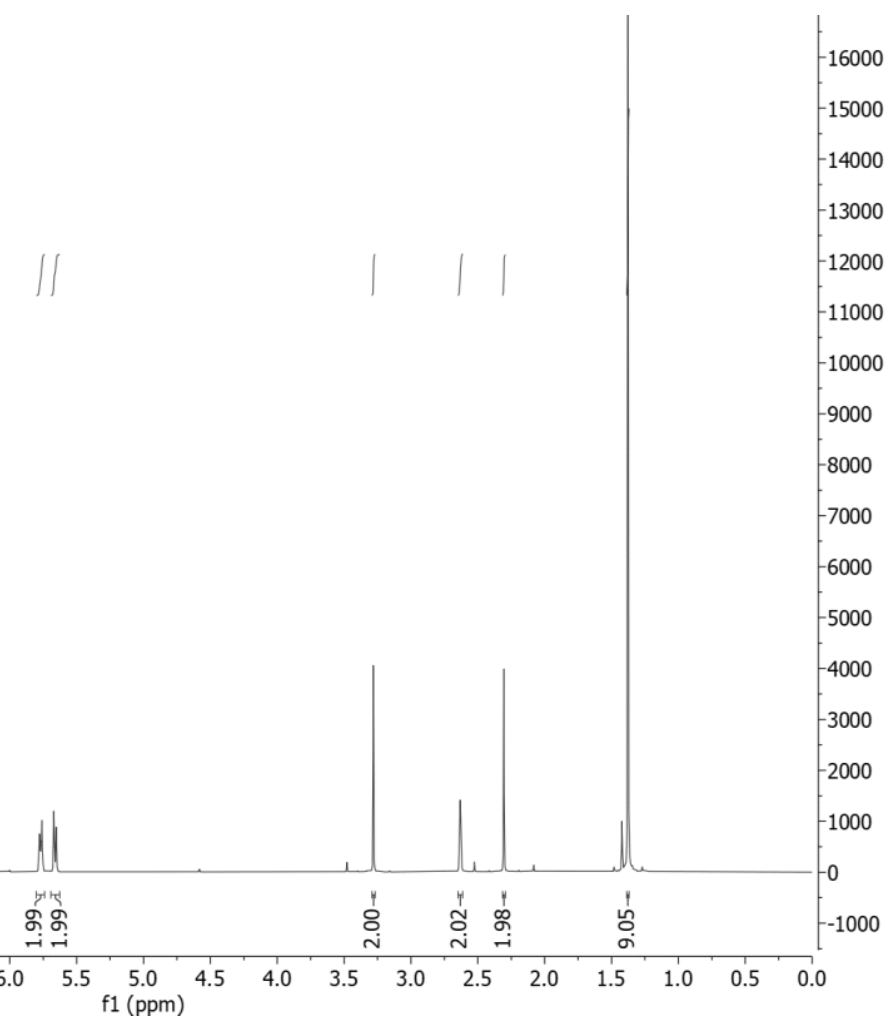

${ }^{13} \mathrm{C}$ NMR of 19

$150.93 \mathrm{MHz}$

CDCl3

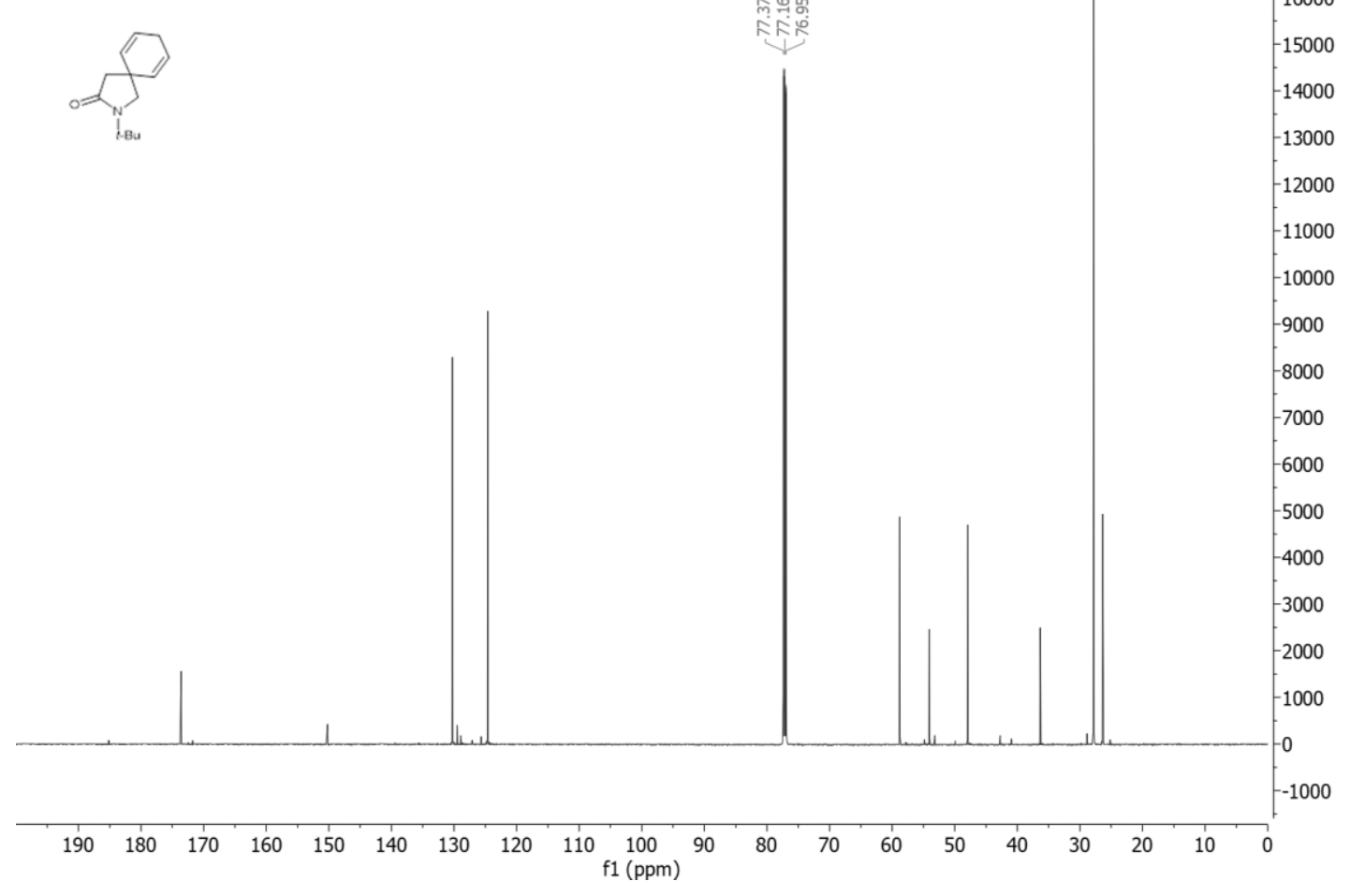


${ }^{1} \mathrm{H}$ NMR of $\mathbf{S 5 1}$

$600.18 \mathrm{MHz}$
$\mathrm{CDCl} 3$
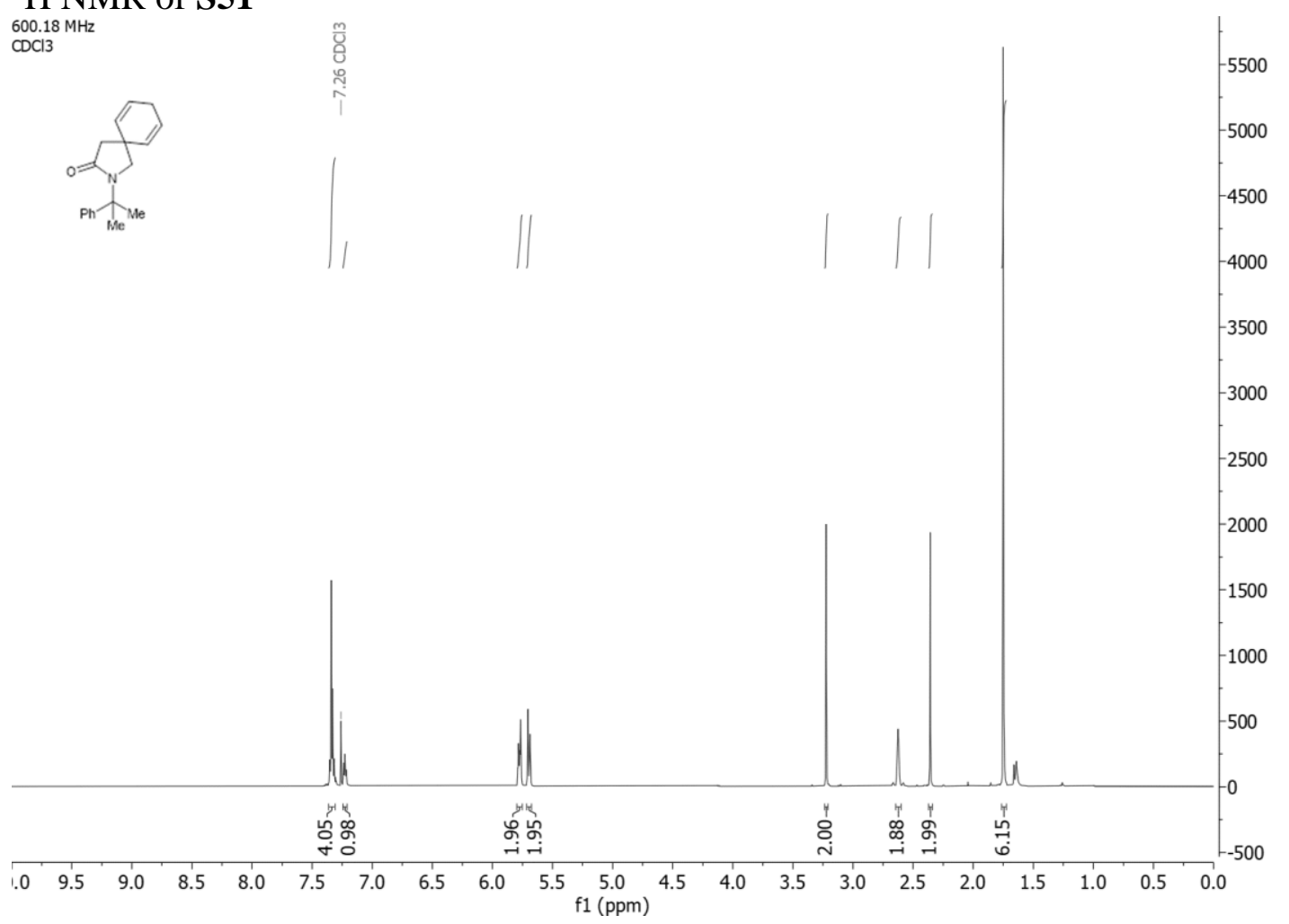

${ }^{13} \mathrm{C}$ NMR of $\mathbf{S 5 1}$

$150.93 \mathrm{MHz}$

$\mathrm{CDCl} 3$

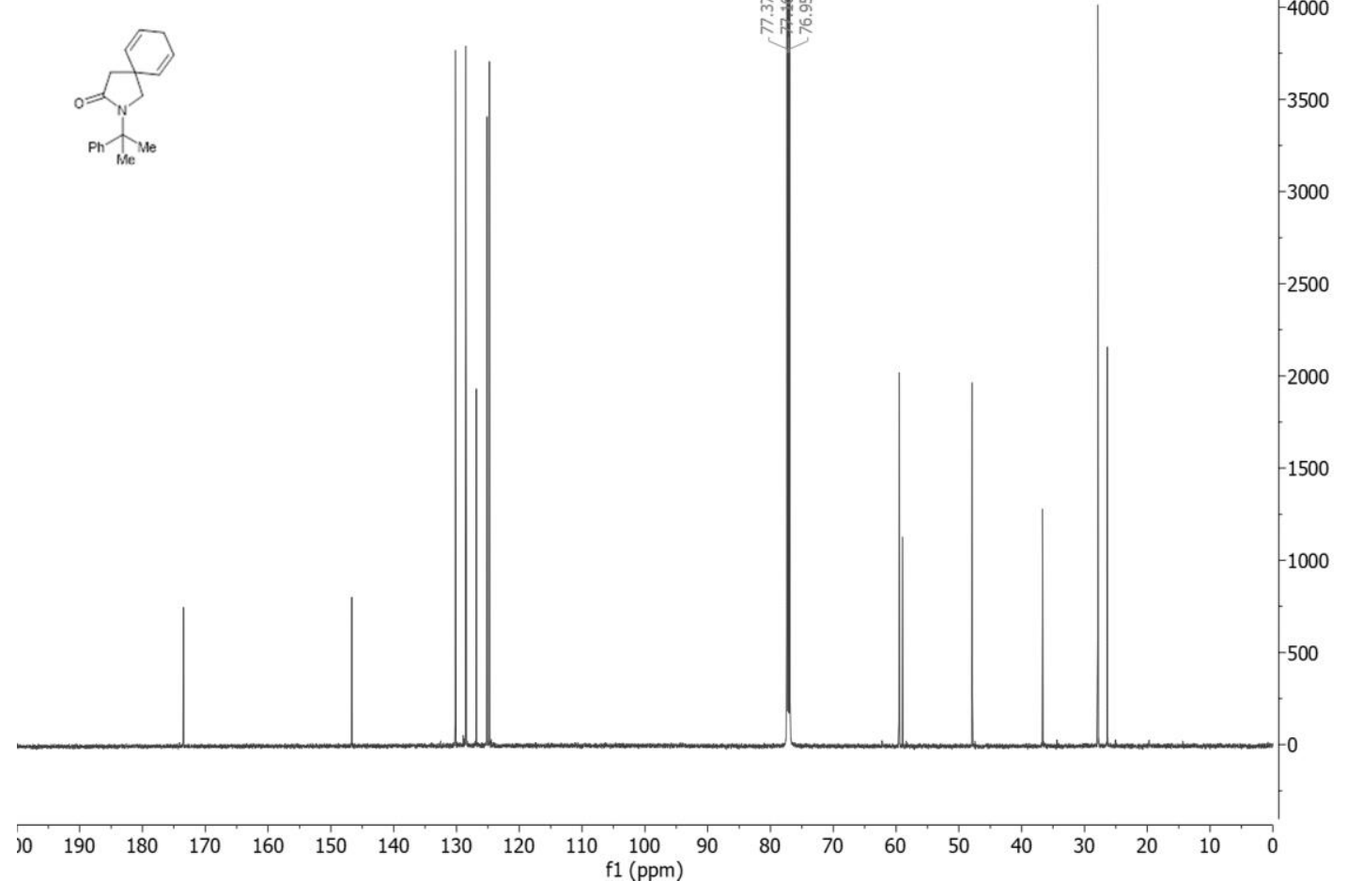


${ }^{1} \mathrm{H}$ NMR of 20 diastereomer 1

$400.15 \mathrm{MHz}$

$\frac{m}{8}$
8
$\searrow$
$i$
$i$

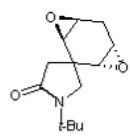

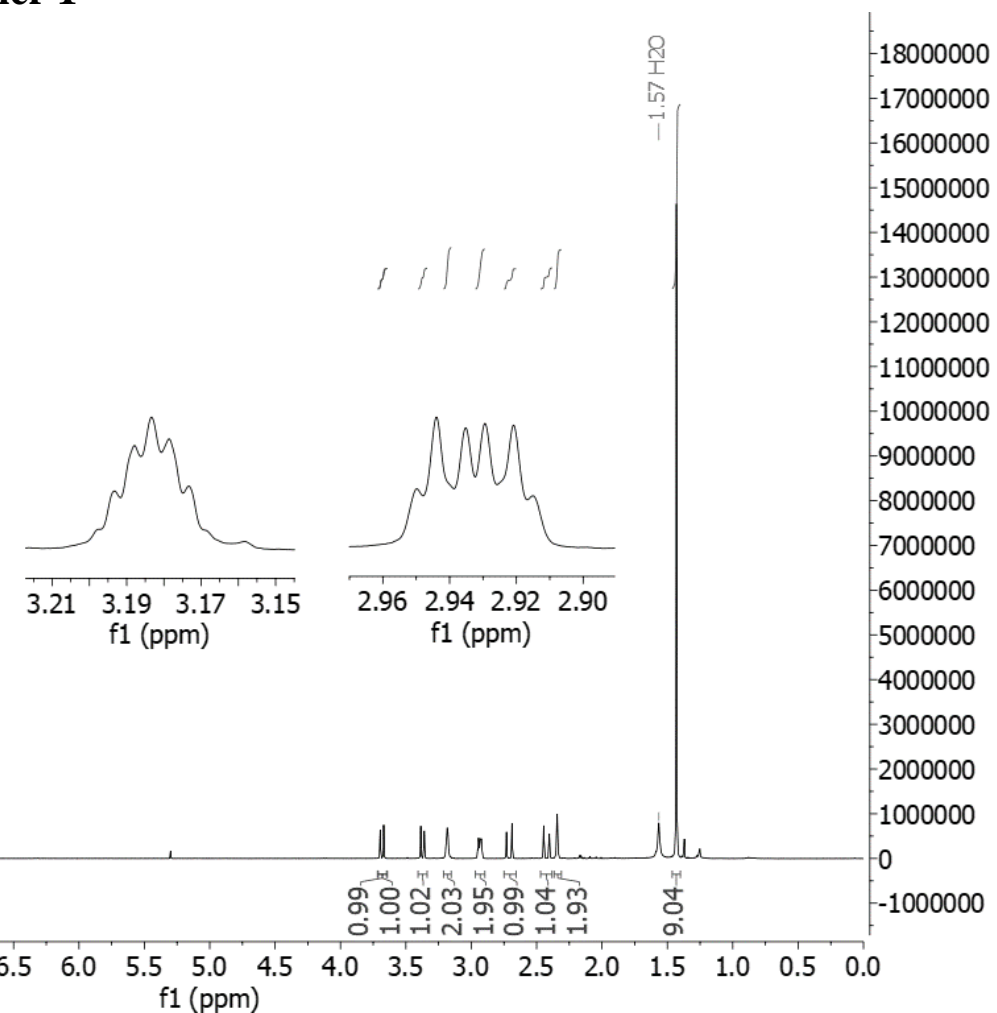

${ }^{13} \mathrm{C}$ NMR of 20 diastereomer 1 $150.93 \mathrm{MHz}$ CDCl3

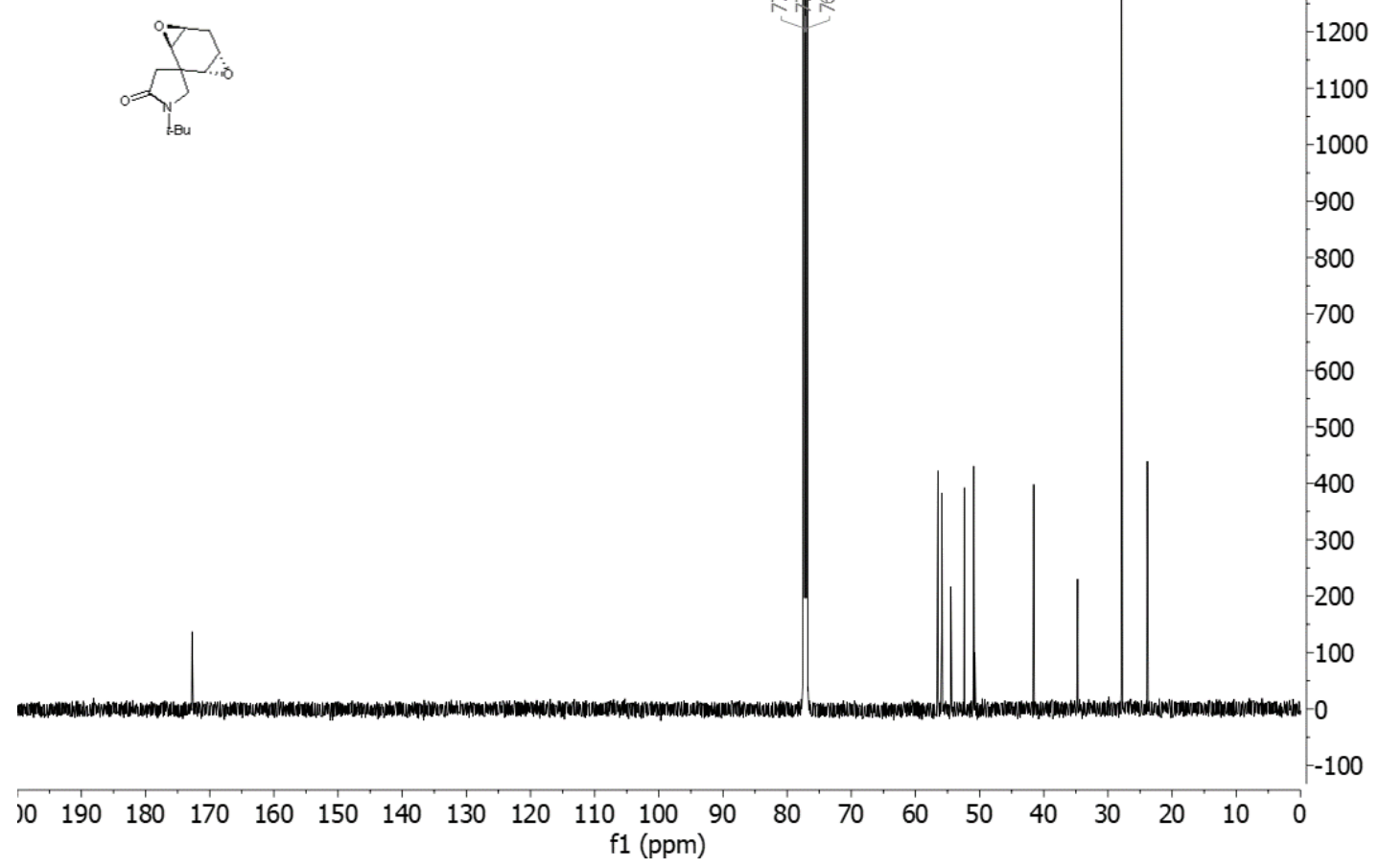


${ }^{1} \mathrm{H}$ NMR of 20 diastereomers 2 and 3

$400.15 \mathrm{MHz}$

$\mathrm{CDCl} 3$

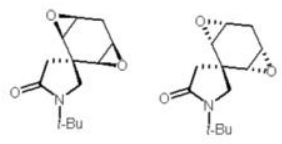

major minor

17000000
-16000000
15000000
-14000000
-13000000
-12000000
-11000000
-10000000
-9000000
-8000000
-7000000
6000000
-5000000
4000000
-3000000
-2000000
-1000000
-0
-1000000
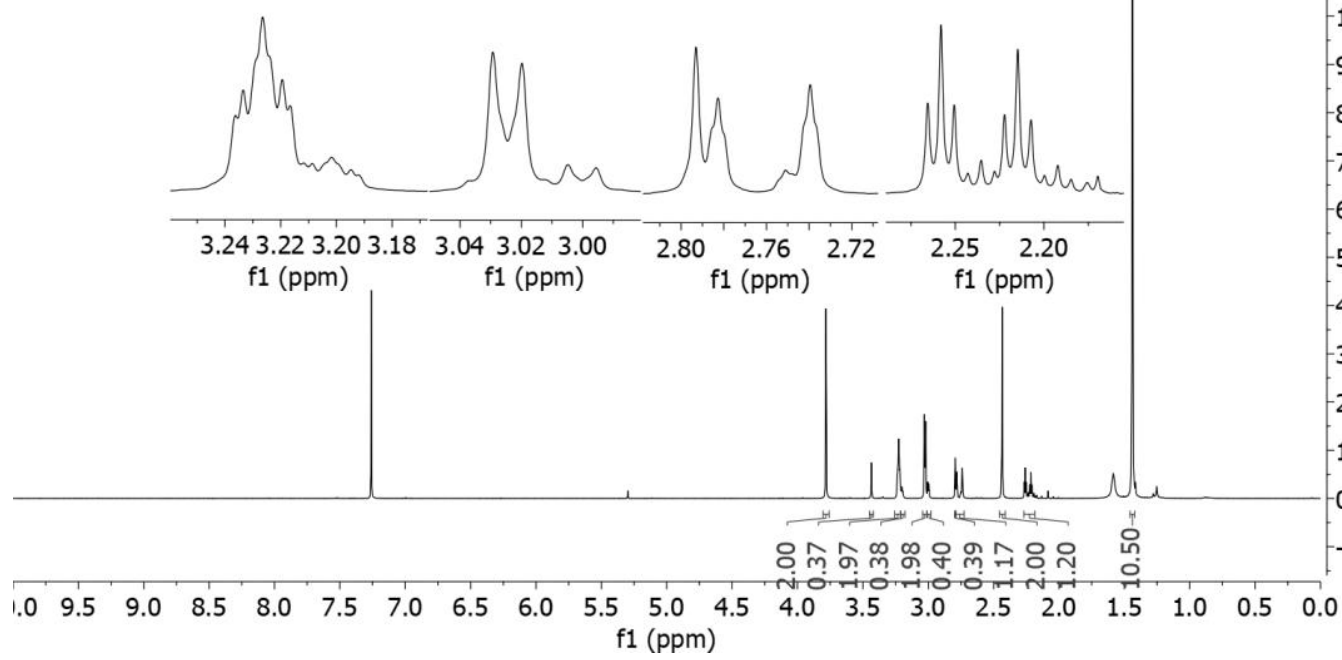

000000

${ }^{13} \mathrm{C}$ NMR of 20 diastereomers 2 and 3 $150.93 \mathrm{MHz}$ $\mathrm{CDCl} 3$

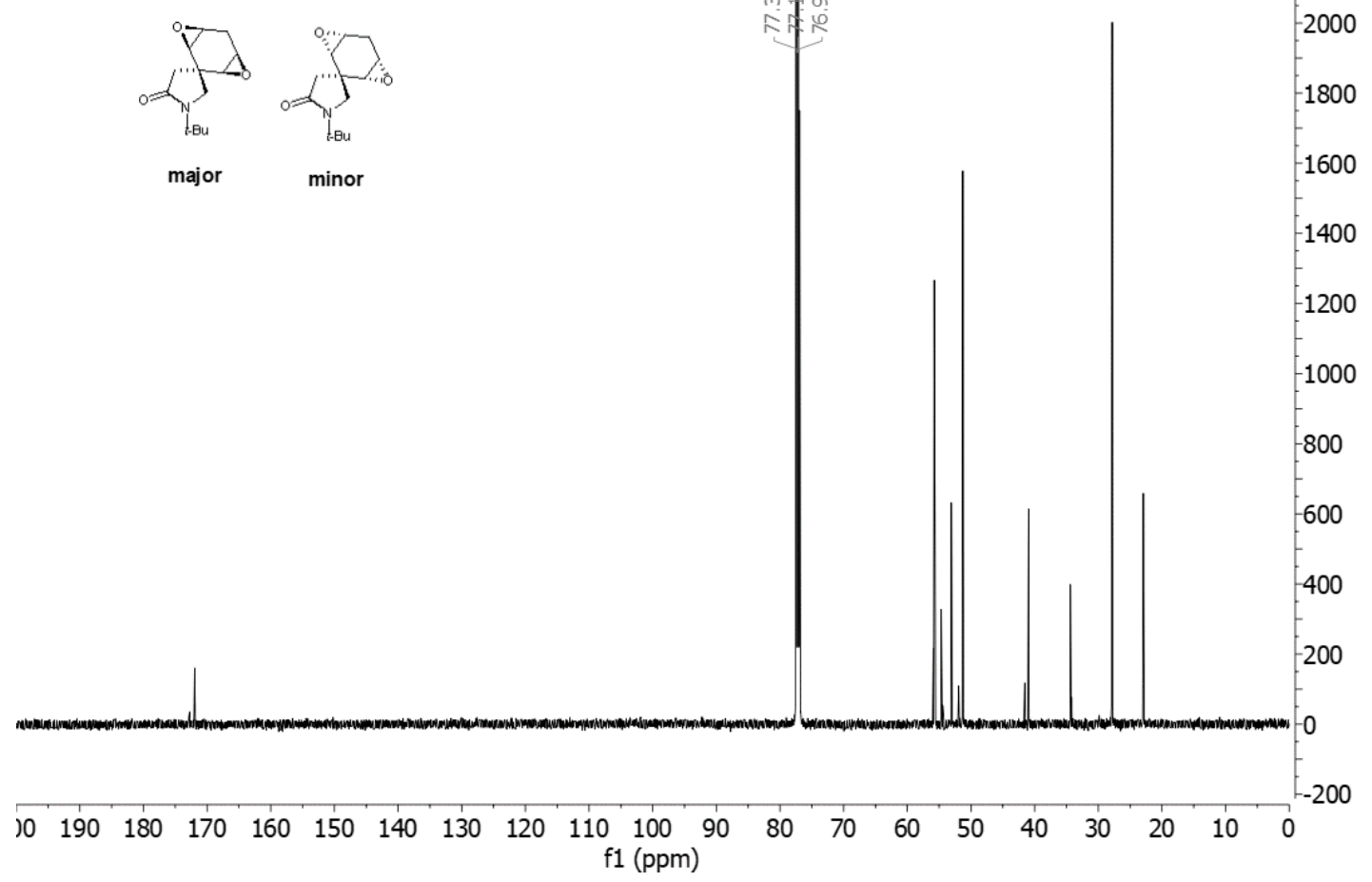


${ }^{1} \mathrm{H}$ NMR of $\mathbf{2 1}$

${ }_{000.18 \mathrm{MHz}}^{6013}$

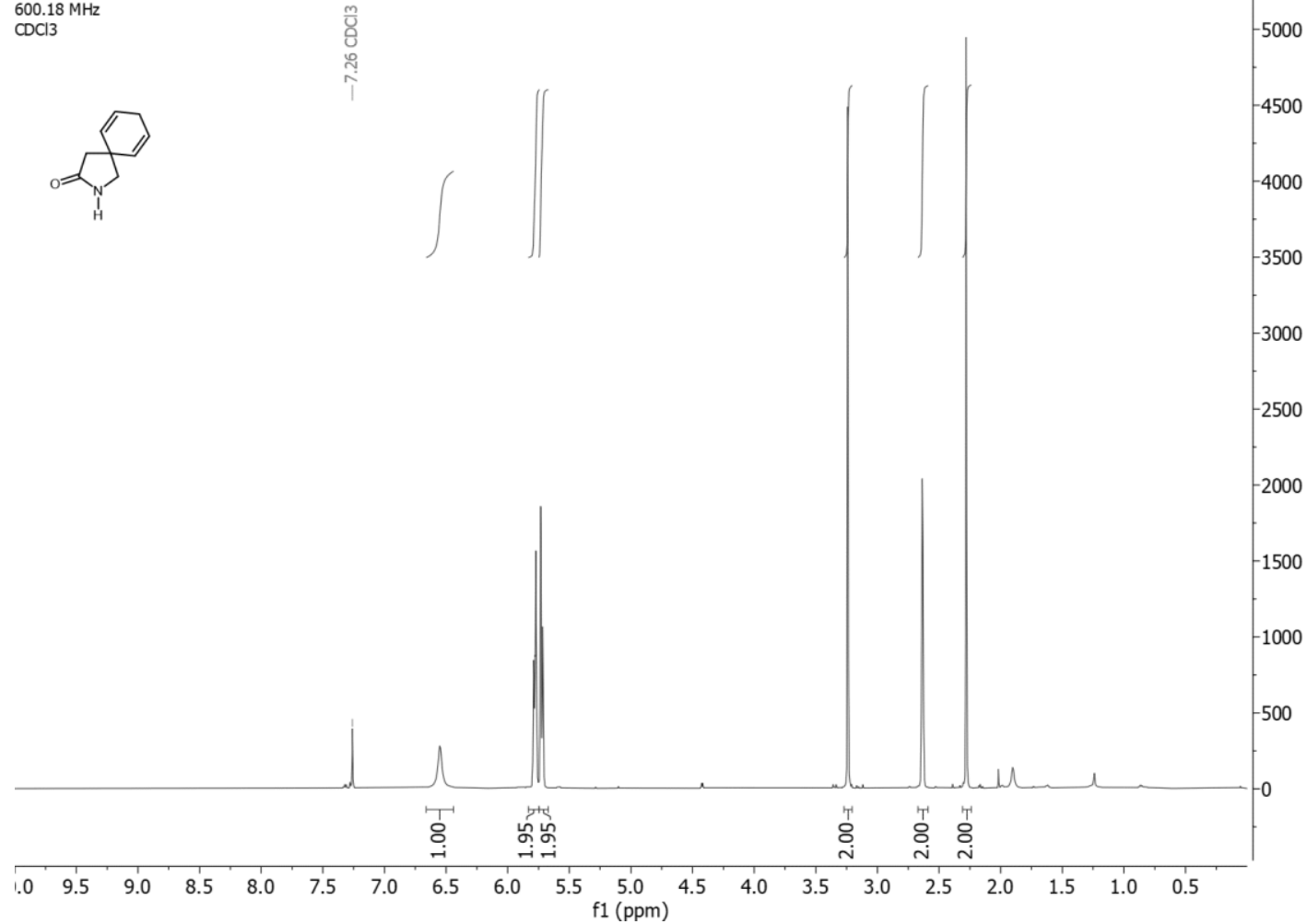

${ }^{13} \mathrm{C}$ NMR of 21

${ }_{150.93} \mathrm{MHz}$

$\mathrm{CDCl} 3$

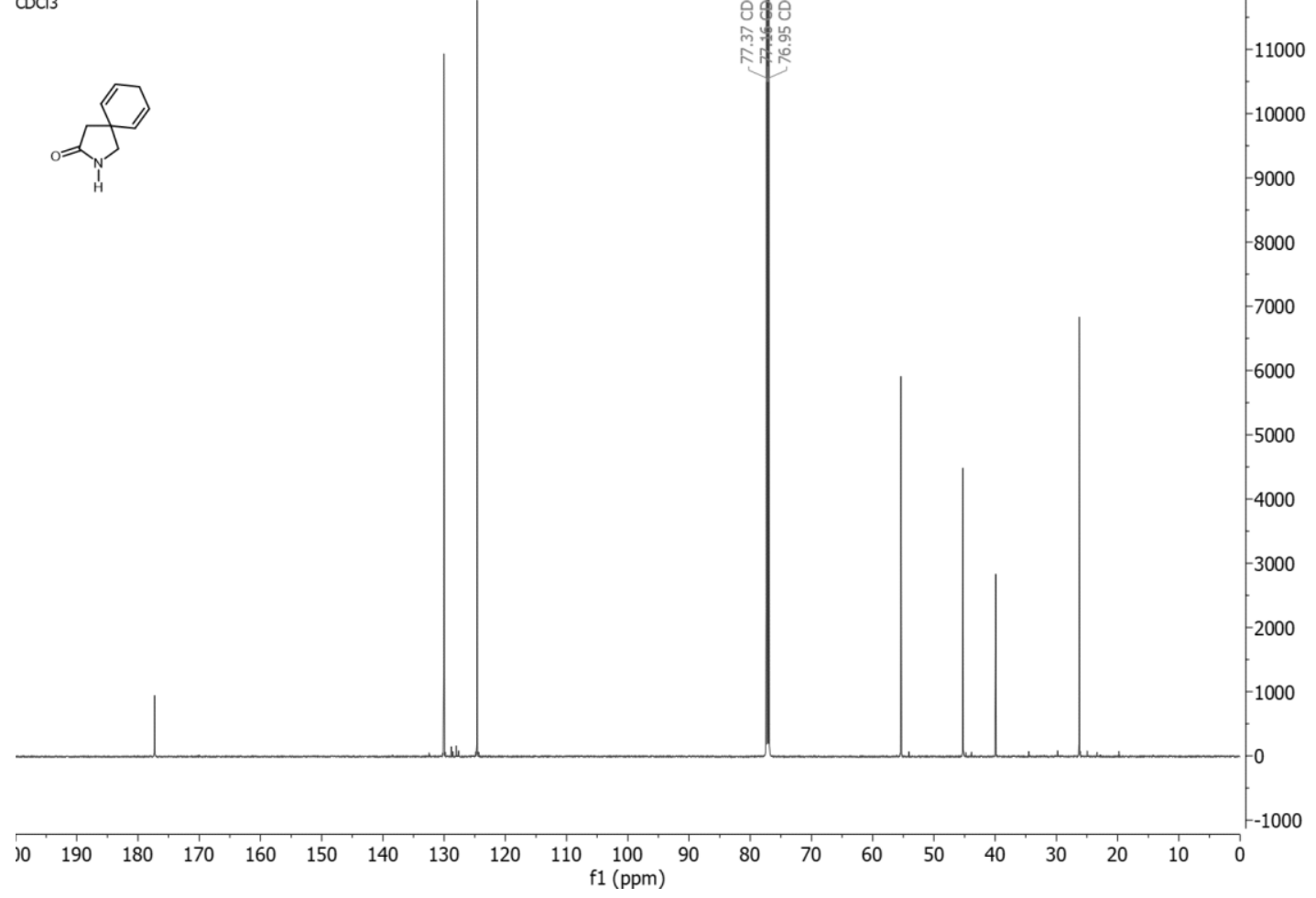


${ }^{1} \mathrm{H}$ NMR of 22

$600.18 \mathrm{MHz}$

0
0
0
0
7
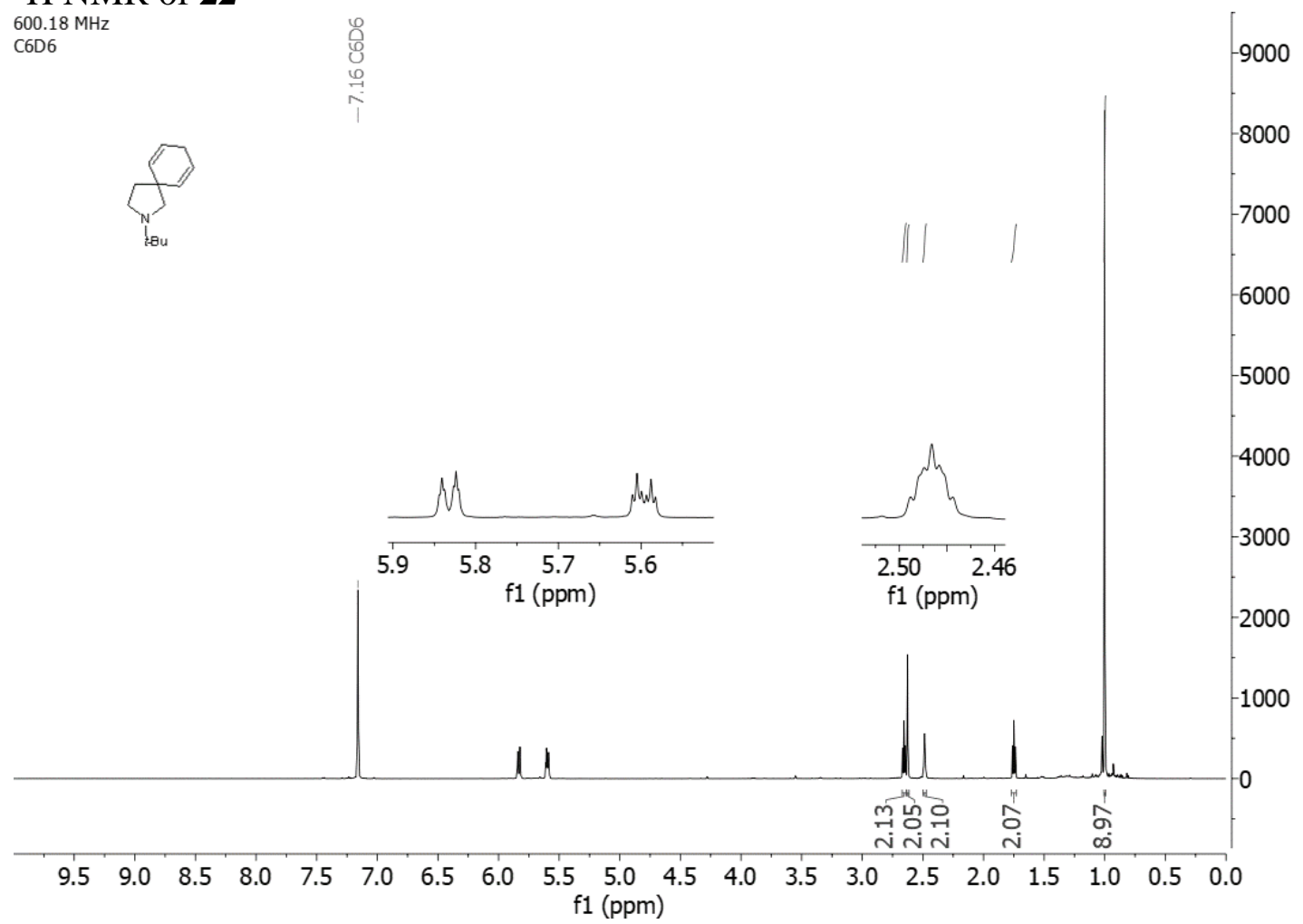

\section{${ }^{13} \mathrm{C}$ NMR of 22}

$150.93 \mathrm{MHz}$

C6D6
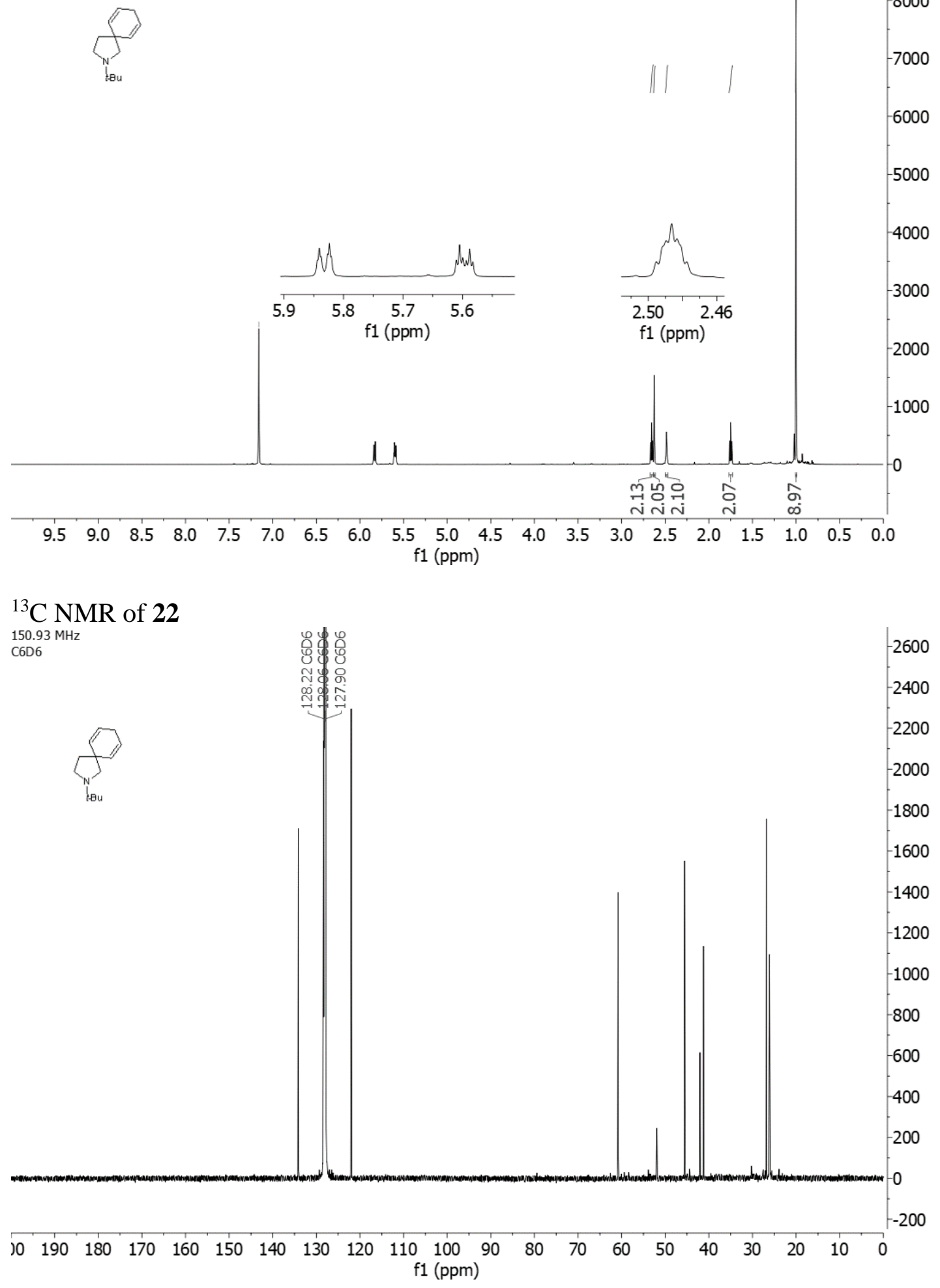
${ }^{1}$ H NMR of 24

$400.15 \mathrm{MHz}$

$\mathrm{CDCl} 3$
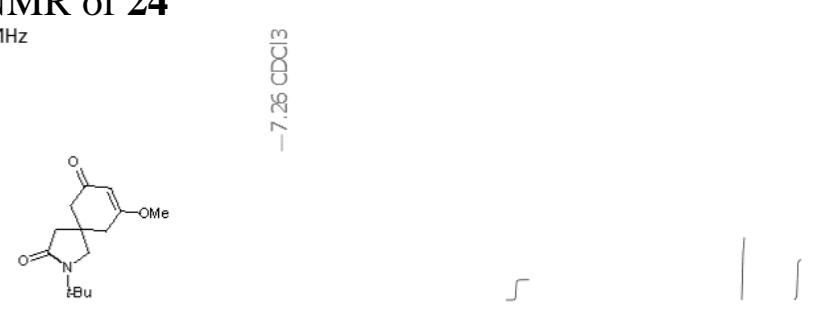

$-3.0 \times 10^{\prime}$

$-2.8 \times 10^{7}$

$-2.6 \times 10^{7}$

$-2.4 \times 10^{7}$

$-2.2 \times 10^{7}$

$-2.0 \times 10^{7}$

$-1.8 \times 10^{7}$

$-1.6 \times 10^{7}$

$-1.4 \times 10^{7}$

$-1.2 \times 10^{7}$

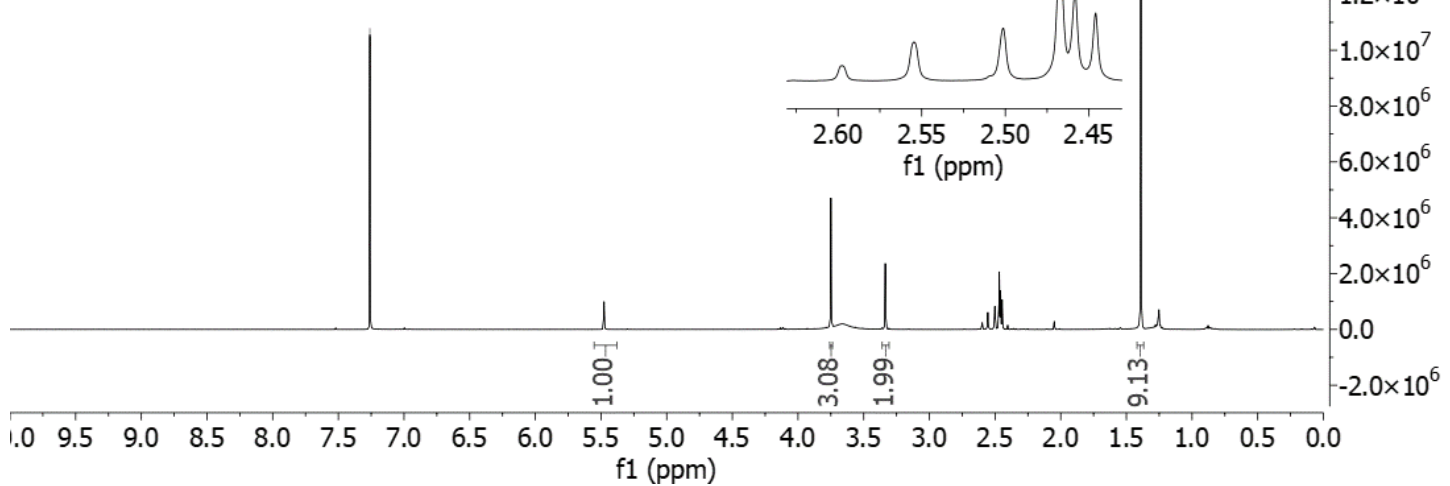

${ }^{13} \mathrm{C}$ NMR of 24

$150.93 \mathrm{MHz}$
$\mathrm{CDCl} 3$

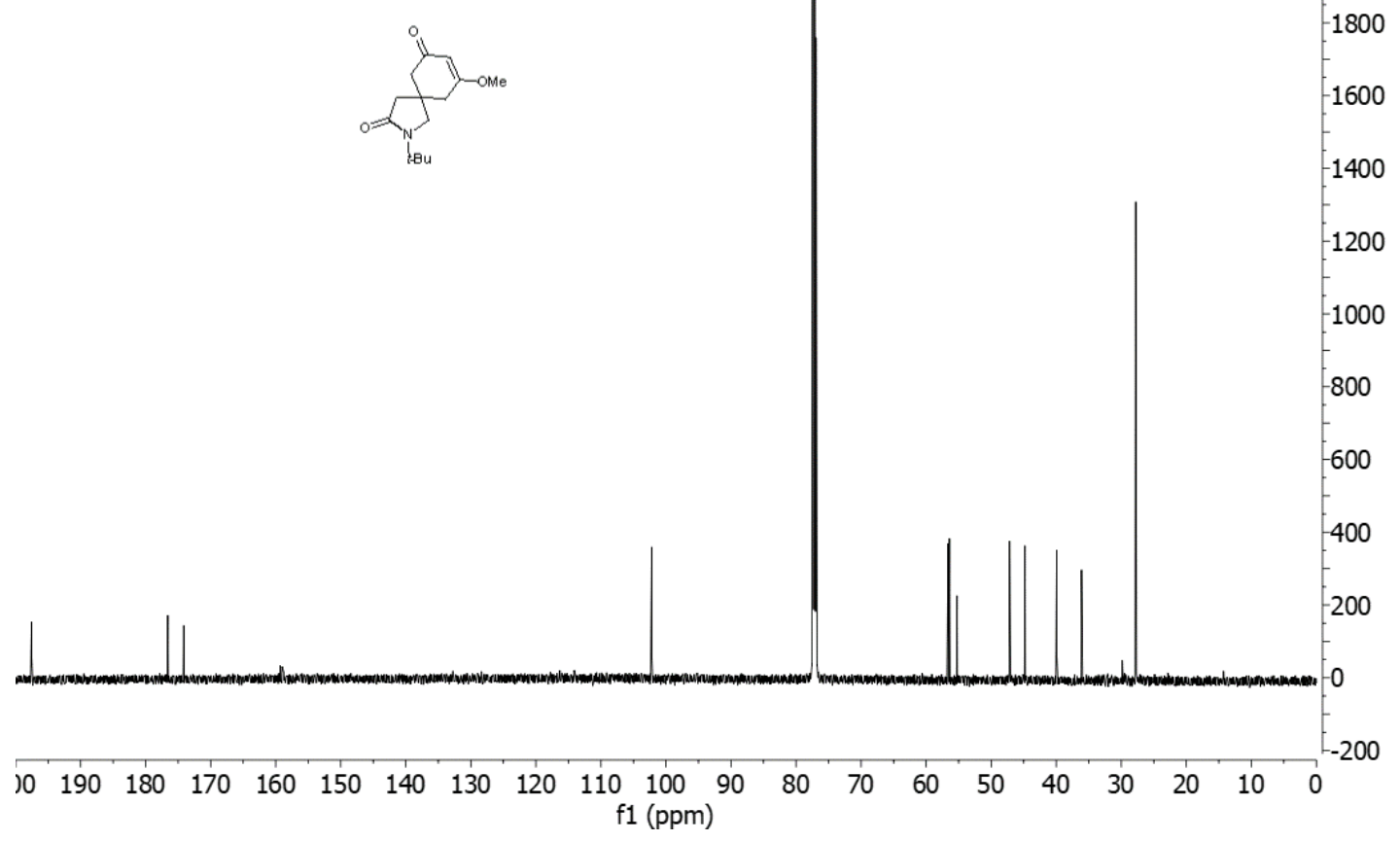


${ }^{1} \mathrm{H}$ NMR of $\mathbf{2 5}$

$400.15 \mathrm{MHz}$
$\mathrm{CDCl} 3$

$m$
$\stackrel{0}{0}$
$\stackrel{0}{\pi}$
$i$

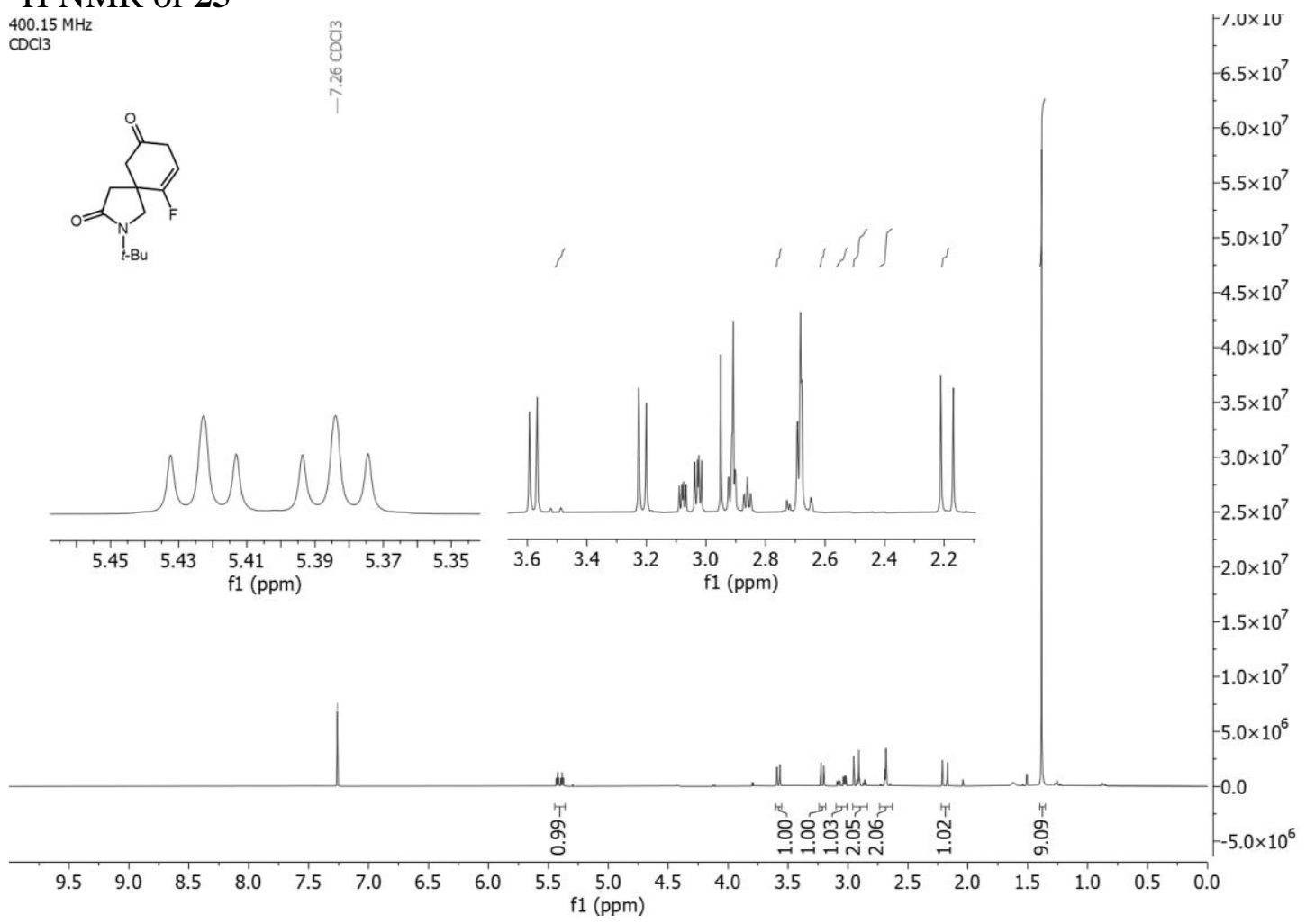

${ }^{13} \mathrm{C}$ NMR of 25 $100.63 \mathrm{MHz}$
$\mathrm{CDCl} 3$
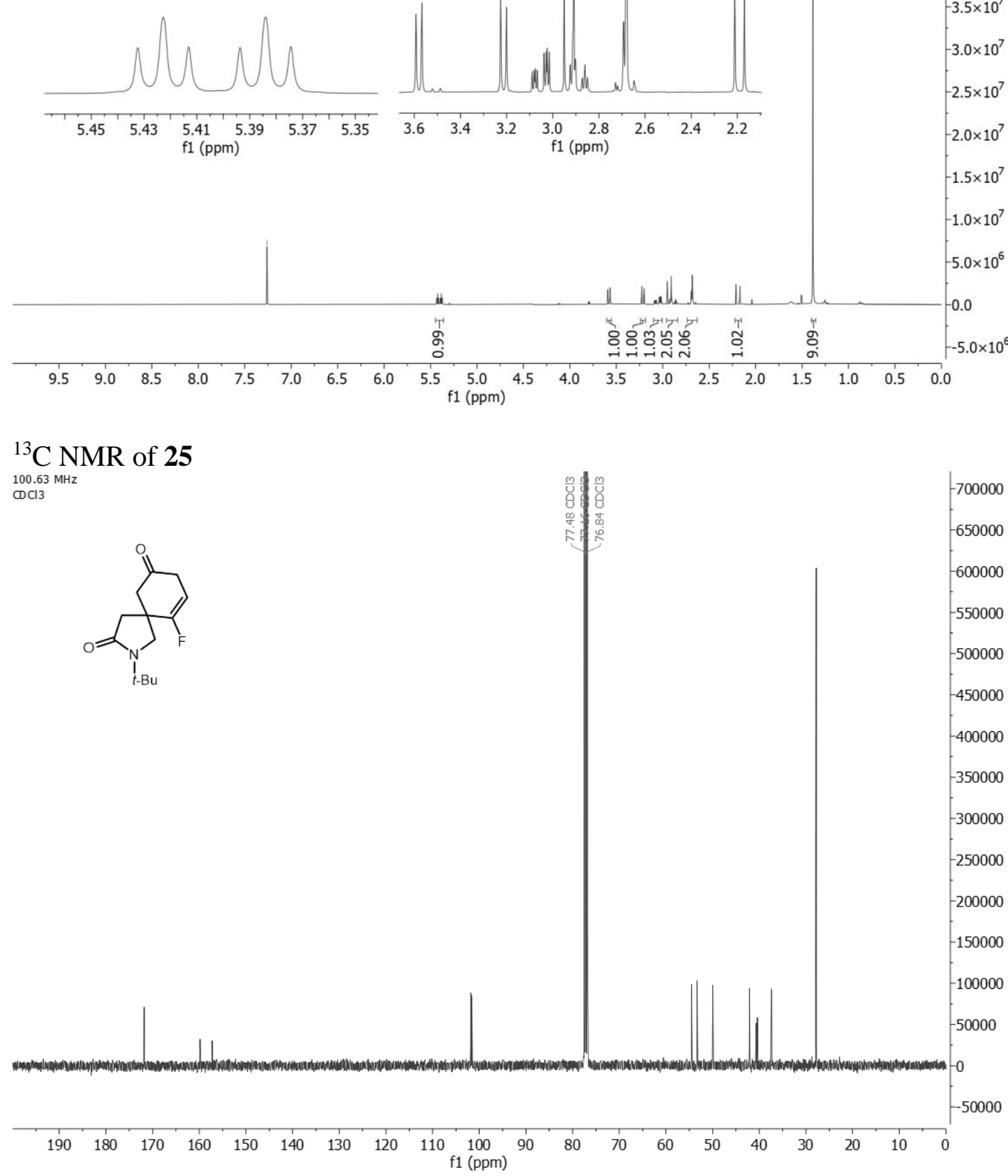

$3.5 \times 10^{7}$

$3.0 \times 10^{7}$

$.5 \times 10^{7}$

$2.0 \times 10^{7}$

$.5 \times 10^{7}$

$.0 \times 10^{7}$ 